University of Rhode Island

DigitalCommons@URI

Open Access Dissertations

2016

\title{
Lipid Nanoparticle Interactions and Assembles
}

Matthew Ryan Preiss

University of Rhode Island, mrp2111@gmail.com

Follow this and additional works at: https://digitalcommons.uri.edu/oa_diss

\section{Recommended Citation}

Preiss, Matthew Ryan, "Lipid Nanoparticle Interactions and Assembles" (2016). Open Access

Dissertations. Paper 459.

https://digitalcommons.uri.edu/oa_diss/459

This Dissertation is brought to you for free and open access by DigitalCommons@URI. It has been accepted for inclusion in Open Access Dissertations by an authorized administrator of DigitalCommons@URI. For more information, please contact digitalcommons-group@uri.edu. 


\section{LIPID NANOPARTICLE INTERACTIONS AND ASSEMBLIES}

BY

MATTHEW RYAN PREISS

A DISSERTATION SUBMITTED IN PARTIAL FULFILLMENT OF THE

REQUIREMENTS FOR THE DEGREE OF

DOCTOR OF PHILOSOPHY

IN

CHEMICAL ENGINEERING

UNIVERSITY OF RHODE ISLAND

2016 


\section{DOCTOR OF PHILOSHOPHY DISSERTATION}

OF

MATTHEW RYAN PREISS

\section{APPROVED:}

Dissertation Committee:

Geoffrey Bothun

Arijit Bose

Stephen Kennedy

Nasser H. Zawia

DEAN OF THE GRADUATE SCHOOL 


\begin{abstract}
Novel liposome-nanoparticle assemblies (LNAs) provide a biologically inspired route for designing multifunctional bionanotheranostics. LNAs combine the benefits of lipids and liposomes to encapsulate, transport, and protect hydrophilic and hydrophobic therapeutics with functional nanoparticles. Functional nanoparticles endow LNAs with additional capabilities, including the ability to target diseases, triggered drug release, controlled therapeutic output, and diagnostic capabilities to produce a drug delivery system that can effectively and efficiently deliver therapeutics while reducing side effects. Not only could LNAs make existing drugs better, they could also provide an avenue to allow once promising non-approved drugs (rejected due to harmful side effects, inadequate pharmacokinetics, and poor efficacy) to be safely used through targeted and controlled delivery directly to the diseased site. LNAs have the potential to be stimuli responsive, delivering drugs on command by external (ultrasound, RF heating, etc.) or internal ( $\mathrm{pH}$, blood sugar, heart rate, etc.) stimuli. Individually, lipids and nanoparticles have been clinically approved for therapy, such as Doxil (a liposomal doxorubicin for cancer treatment), and diagnosis, such as Feridex (an iron oxide nanoparticle an MRI contrast enhancement agent for liver tumors).
\end{abstract}

In order to engineer these multifunctional LNAs for theranostic applications, the interactions between nanoparticles and lipids must be better understood. This research sought to explore the formation, design, structures, characteristics, and functions of LNAs. To achieve this goal, different types of LNAs were formed, 
specifically magnetoliposomes, bilayer decorated LNAs (DLNAs), and lipid-coated magnetic nanoparticles (LMNPs).

A fluorescent probe was embedded in the lipid bilayer of magnetoliposomes allowing the local temperature and membrane fluidity to be observed. When subjected to an electromagnetic field that heated the encapsulated iron oxide nanoparticles encapsulated in the lipid bilayer, the local temperature and membrane fluidity could be observed.

DLNAs were encapsulated with different sized nanoparticles and concentrations in order to observe the effect of the bilayer nanoparticles on the lipid bilayer's phase behavior and leakage. Two different sized nanoparticles were used, a 2 $\mathrm{nm}$ gold nanoparticle (GNP) much smaller than the thickness of the bilayer and a $4 \mathrm{~nm}$ GNP near the thickness of the lipid bilayer. The $2 \mathrm{~nm}$ GNPs were shown to affect the lipid bilayer differently than the $4 \mathrm{~nm}$ GNP. Specifically, the two nanoparticles altered the phase behavior and leakage differently in a temperature dependent fashion, demonstrating that embedded nanoparticle size can be used induce or inhibit bilayer leakage.

A dual solvent exchange method was used to control the lipid surface composition of an iron oxide nanoparticle with a cationic lipid and a polyethylene glycol (PEG) lipid to produce lipid coated magnetic nanoparticles (LMNPs). PEG is well known for its ability to enhance the pharmacokinetics of nanostructures by preventing uptake by the immune system. By controlling the lipid surface composition, the surface charge and PEG conformation can be controlled which 
allowed the LMNPs to be used as an MRI contrast agent and a delivery system for siRNA that could be triggered with temperature. 


\section{ACKNOWLEDGMENTS}

I would like to extend my deepest gratitude to my whole family: Mom, Dad, Jenn, Jess, Amy, Tom, Scott, Ben, Marcus, Jake, Jessica Rose, Jackson, Jeff, Liz, and Chris without your loving support, I would not have been able to accomplish this. I love you with all my heart.

I would like to especially recognize my Father, who is my best friend and role model. Without your support, guidance, time, and love, I would not be the person I am today. You have been the most influential person in my life. I hope someday I can become the man you are. Everything I accomplish I owe to you. Thank you for everything. I cannot express how much I love you.

I would like to also thank my Mother for her support in all aspects of my life. No matter what time of day or night, you have always been there for me and I cannot thank you enough for that. I truly appreciate everything you have done for me. Thank you for always being there willing to listen and help when I needed it most. I love you with all my heart and am grateful to have a mother like you.

I would also like to extend a heartfelt thank you to Dr. Geoffrey Bothun. You are not just a teacher or mentor to me, you are also a friend. Thank you for allowing me to be your graduate student and having the opportunity to learn from you. You have had a profound influence on my life, not just as a student and researcher, but also

as a person. I am a better person because of you. I truly appreciate your willingness to always been there for me, regardless if it is school related or not. You are and always will be one of the most influential people in my life. You have touched my life more 
than you will ever know or realize. I will always have a special place in my heart for you. Thank you for everything.

I would like to acknowledge Providence College, Columbia University, and University of New Hampshire School of Law and their faculty, the education I have received from each of these institutions have had an enormous influence on me as a person. I especially would like the University of Rhode Island and the Department of Chemical Engineering for giving me the opportunity to learn from such great faculty members and allowing me to study for my Ph.D. I would like to especially thank the following professors for their influence on me: Dr. Harry Knickle (my M.S. advisor), Dr. Stanley Kowalski (ITTI and UNH Law), and Jon Cavicchi (UNH Law). 


\section{PREFACE}

This dissertation was prepared in manuscript format. Chapter 1 is an overview into the various different lipid-nanoparticle assemblies (LNAs) structures that can be formed combining lipids and nanoparticles for therapeutic and diagnostic (theranostic) applications. Chapter 2 investigates LNAs responsive to stimuli, such as magnetic, temperature, $\mathrm{pH}$, light, ultrasound, etc., which can allow them to be used for triggered and controlled release of therapeutics. Chapters 1 and 2 provide a summary of the current status of LNA research and development. Chapter 3 is a manuscript related to measuring the fluidity of the lipid bilayer of a magnetoliposomes (liposomes with iron oxide encapsulated in its aqueous core) when the nanoparticles are heated with an electromagnetic field at radio frequency. Chapter 4 is a manuscript that investigated the effect nanoparticle size and concentration on the phase behavior and permeability of bilayer decorated LNAs (DLNAs). Chapter 5 is a manuscript that examines how lipid surface concentration of an iron oxide nanoparticle effects the MRI relaxivity and siRNA binding and release.

The first chapter, entitled "Liposome-Nanoparticle Assemblies", is Chapter 11 (pg. 273-307) in the book Bionanotechnology: Biological Self-Assembly and its Applications published by Caister Academic Press (Norfolk, UK) in 2013. This chapter was invited submission by the editor, Bernd H.A. Rehm. Also, flatteringly, the front cover of the book (displayed on the Chapter 1 title page) was selected from Figure 1-2 that we submitted. 
The second chapter, entitled "Stimuli-Responsive Liposome-Nanoparticle Assemblies", was an invited article published in Expert Opinion on Drug Delivery in 2011 (Expert Opinion on Drug Delivery 8(8) 1025-1040 (2011)).

The third chapter, entitled "Local Heating in Magnetite NanoparticleLiposome Dispersions via Fluorescence Anisotropy”, was published in the Journal of Colloid and Interface Science in 2011 (Journal of Colloid and Interface Science 357(1) 70-74 (2011)).

The fourth chapter, entitled "Hydrophobic Nanoparticles Embedded in Liposomes Modify the Thermal Release Behavior of Encapsulated Carboxyfluorescein", is a manuscript currently in preparation for submission to ACS Nano.

The fifth chapter, entitled "MRI Relaxivity and siRNA Binding Capacity of Lipid-Coated Magnetic Nanoparticles Controlled by PEG Confirmation”, is a manuscript currently in preparation for submission to Nano Letters. 


\section{TABLE OF CONTENTS}

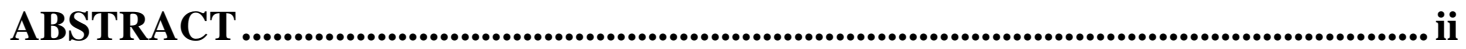

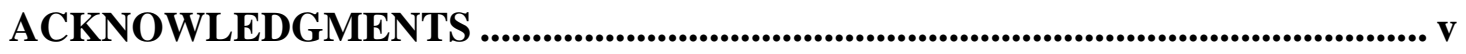

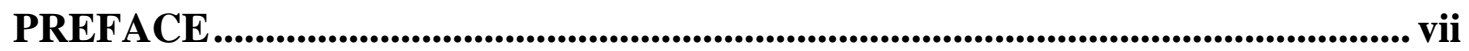

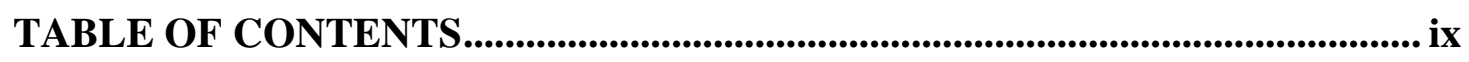

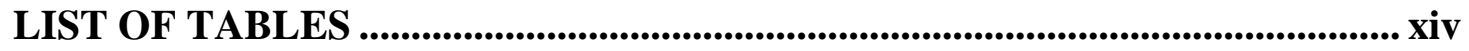

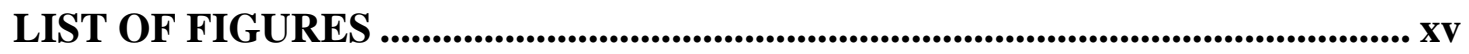

CHAPTER 1 .

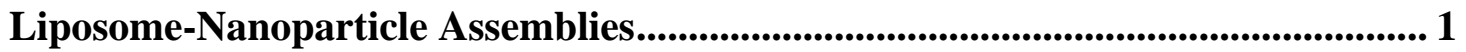

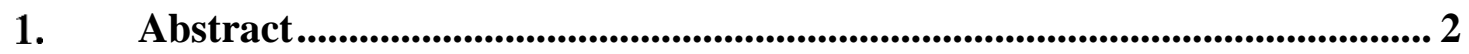

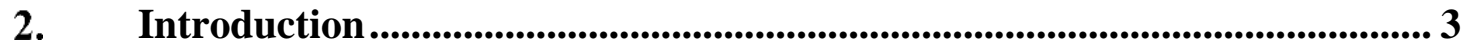

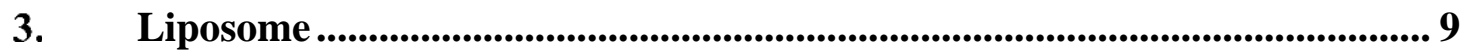

4. Nanoparticles ................................................................................................... 13

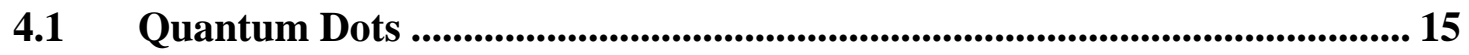

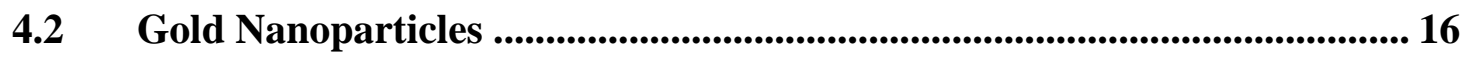

4.3 Superparamagnetic Iron Oxide Nanoparticles ........................................ 17

5. Formation, Structure, and Design Strategies ................................................ 18

5.1 Encapsulated Liposome-Nanoparticle Assembly .................................... 24

5.2 Bilayer-Decorated Liposome-Nanoparticle Assembly ................................ 31

5.3 Surface-Coupled Liposome-Nanoparticle Assembly and Complexation.. 34

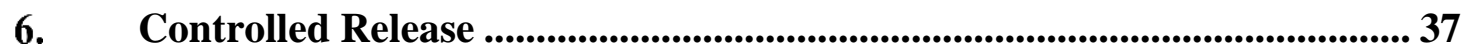

6.1 Gold Nanoparticles and Photothermal Effects......................................... 38

6.2 Iron Oxide Nanoparticles and Alternating Magnetic Fields...................... 41 
7. Targeted Therapy …........................................................................................... 43

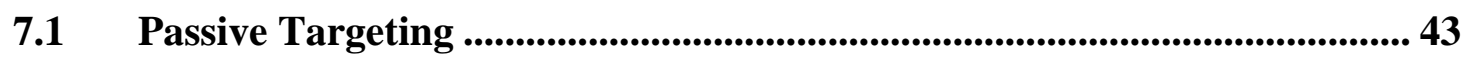

7.2 Active Targeting ........................................................................................................ 47

7.2.1 Ligand Targeting....................................................................................................... 48

7.2.2 Magnetic Drug Targeting ......................................................................................... 51

8. Diagnostics and Imaging .................................................................................... 53

9. Hyperthermia ..................................................................................................................... 56

10. In vivo and in vitro Biomedical Applications ..................................................... 58

10.1 Cellular Uptake and Drug Delivery ............................................................58

10.2 Drug Delivery and Hyperthermia ………............................................................. 59

11. Conclusion and Future Outlook ........................................................................ 60

12. References .......................................................................................................................6 61

CHAPTER 2 ......................................................................................................................... 84

Stimuli-Responsive Liposome-Nanoparticle Assemblies................................................ 84

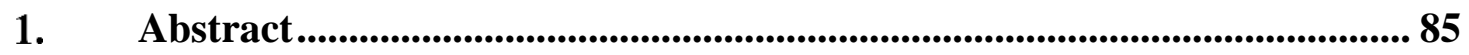

1.1 Introduction:............................................................................................................ 85

$1.2 \quad$ Areas Covered: .................................................................................................... 85

$1.3 \quad$ Expert Opinion:...................................................................................................... 85

2. Introduction ............................................................................................................... 87

$2.1 \quad$ Liposomes …............................................................................................................... 92

2.2 Gold and iron oxide nanoparticles .................................................................. 95

2.3 Nanoparticle-mediated hyperthermia..................................................................... 97

3. LNA Formation and Structure .......................................................................... 98 


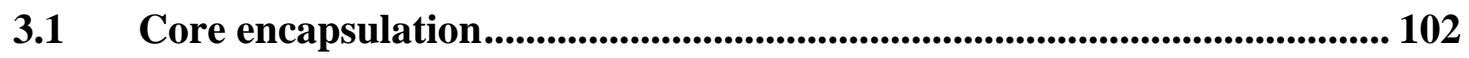

3.2 Bilayer embedment ................................................................................... 105

3.3 Surface decoration and complexation ................................................................. 107

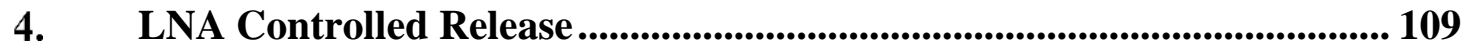

4.1 Gold nanoparticles and photothermal effects............................................ 110

4.2 Iron oxide nanoparticles and alternating magnetic field effects............... 111

5. LNA Performance In vitro and In vivo .................................................. 113

5.1 Cellular uptake and drug delivery ................................................................... 113

$5.2 \quad$ Drug delivery and hyperthermia ................................................................... 114

6. Expert Opinion ........................................................................................................... 116

7. References .............................................................................................................. 119

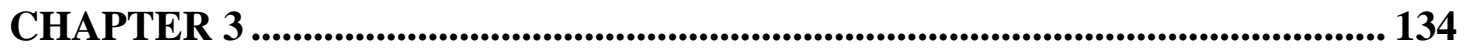

Local Heating in Magnetite Nanoparticle-Liposome Dispersions via Fluorescence Anisotropy.................................................................................................................... 134

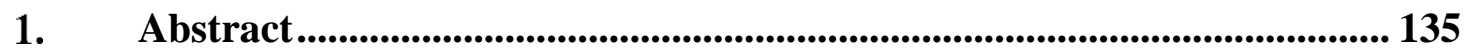

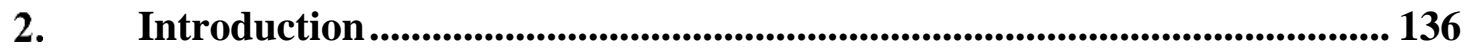

3. Materials and methods ..................................................................................... 139

3.1 Chemicals .............................................................................................................. 139

3.2 Preparation of $\mathrm{Fe}_{3} \mathrm{O}_{4}$ nanoparticle-liposome dispersions............................ 139

3.3 Cryogenic transmission electron microscopy (cryo-TEM) ........................ 140

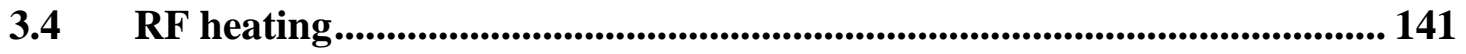

4. Results and discussion .................................................................................. 142

$4.1 \quad$ Nanoparticle RF-heating ......................................................................................... 142 
4.2 Cryo-TEM and DLS analysis...................................................................... 143

4.3 DPH anisotropy as a function of temperature and RF-heating................ 145

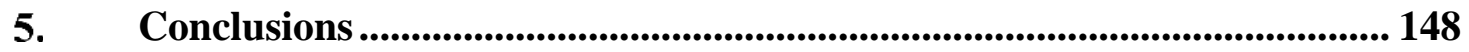

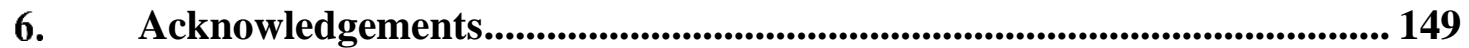

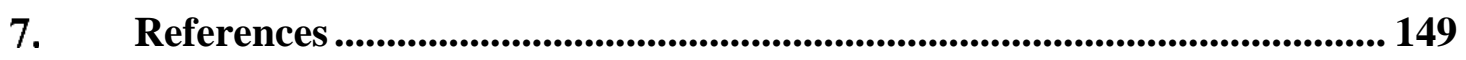

CHAPTER 4 ........................................................................................................................... 151

Hydrophobic Nanoparticles Embedded in Liposomes Modify the Thermal

Release Behavior of Encapsulated Carboxyfluorescein ............................... 151

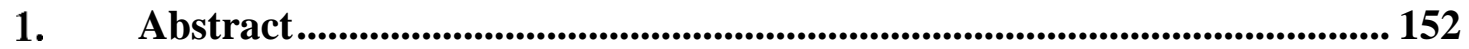

2. Introduction ......................................................................................................... 153

3. Methods and Materials....................................................................................... 156

3.1 Chemicals and Materials........................................................................................... 156

3.2 Gold Nanoparticle Synthesis.............................................................................. 157

$3.3 \quad$ Nanoparticle Fractionation..................................................................................... 157

$3.4 \quad$ Nanoparticle Characterization. ........................................................................... 158

3.5 Liposome and DLNA Preparation......................................................................... 159

3.6 Fluorescence Leakage Studies.............................................................................. 160

3.7 Differential Scanning Calorimetry..................................................................... 162

3.8 Cryogenic Transmission Electron Microscopy (Cryo-TEM)..................... 162

4. Results and Discussion.................................................................................... 162

5. Conclusions ......................................................................................................... 174

6. Acknowledgements................................................................................................... 175

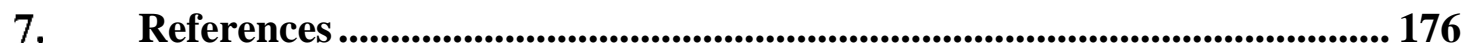


CHAPTER 5

MRI Relaxivity and siRNA Binding Capacity of Lipid-Coated Magnetic

Nanoparticles Controlled by Polyethylene Glycol Confirmation ............ 182

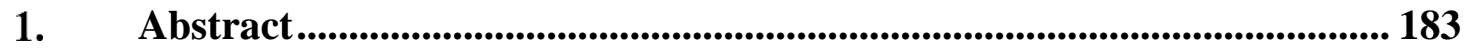

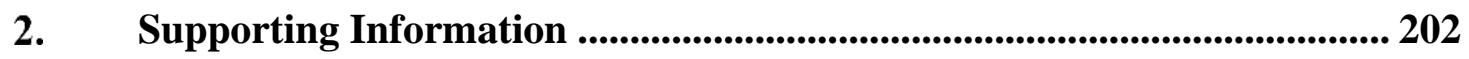

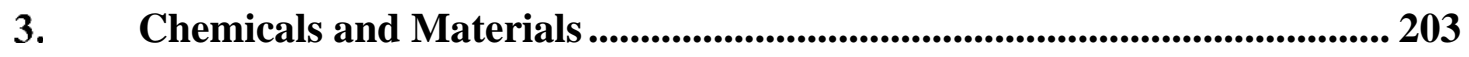

4. Experimental Section ....................................................................................... 204

4.1 Lipid Coated Magnetic Nanoparticle (LMNP) Formation ...................... 204

4.2 Cryogenic Transmission Electron Microscopy (Cryo-TEM)................... 205

4.3 Dynamic Light Scattering (DLS) ............................................................ 205

4.4 Heating with Alternating Current Electromagnetic Field (AC EMF)

Operating at Radio Frequency $(\mathrm{RF})$.................................................. 206

5. Small Interfering RNA (siRNA) Experiments........................................... 207

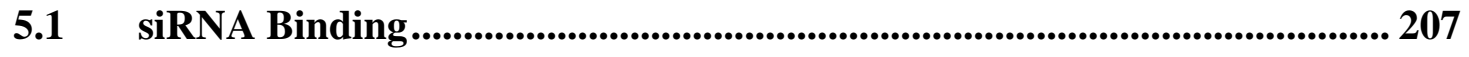

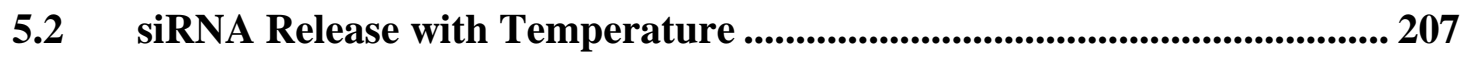

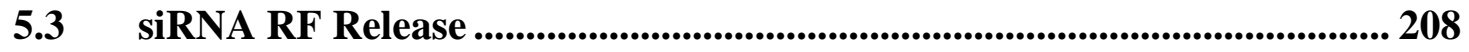

6. Magnetic Resonance Imaging (MRI) .......................................................... 209

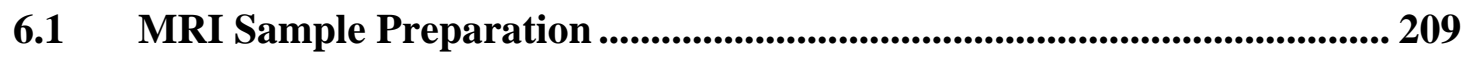

6.2 MRI Methods for $\mathbf{r}_{2}$ Relaxation ............................................................ 209

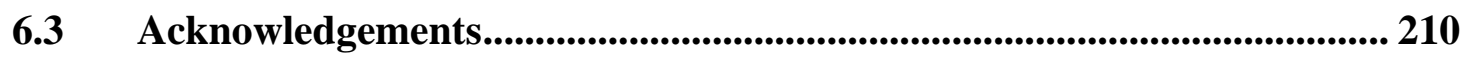

7. References ..................................................................................................... 210 


\section{LIST OF TABLES}

Table 1-1. FDA-approved liposomal formulations............................................................. 7

Table 1-2. FDA-approved therapeutic nanoparticles..................................................... 8

Table 1-3. Review of LNAs formed with gold (Au) nanoparticles. .................................. 21

Table 1-4. Review of LNAs formed with iron oxide nanoparticles.................................... 22

Table 2-1. Reported LNAs based on Au NPs since 2008............................................... 100

Table 2-2. Reported LNAs based on iron oxide NPs since 2008..................................... 101

Table 4-1. Concentration and volume fraction of GNPs loaded into D-LNAs.................... 164

Table 4-2. DPPC and D-LNA lipid phase behavior and CF leakage temperatures............. 169

Table 5-1 Parameters used for calculation of Flory radius, PEG footprint, distance between

PEG groups, area occupied by PEG, PEG chain length, and LMNP diameter......... 189

Table 5-2. Calculated PEG length, PEG footprint, and Calculated and Measured DLMNP. ... 192 


\section{LIST OF FIGURES}

Figure 1-1. Schematic and cryogenic transmission electron micrograph of dipalmitoylphosphatidylcholine liposomes (DPPC). 10

Figure 1-2. Schematics and cryogenic transmission electron micrograph of liposomenanoparticle assemblies

Figure 1-4. Changes in bilayer decoration mechanism of D-LNAs with increasing nanoparticle diameter. 32

Figure 1-5. Remote dye release triggered by near IR laser illumination from C-LNAs. 39

Figure 1-6. Proposed 'plasmonic nanobubble' release mechanism of encapsulated molecules from E-LNAs prepared with gold nanoparticles.

Figure 1-7. A multifunctional E-LNA for cancer thermo-chemotherapy from Pradhan et al. 49

Figure 1-8. Combined imaging and doxorubicin release from D-LNAs prepared with hydrophobic quantum dots (green). 55

Figure 2-1. Schematics and TEM micrographs of liposome-nanoparticle assemblies. 91

Figure 2-2. Conceptualization of a multifunctional liposome-nanoparticle assembly. .92

Figure 2-3. Change in bilayer embedment as a function of changing nanoparticle size. 107

Figure 2-4. Controlled release from a decorated liposome-nanoparticle assembly under the influence of an alternating current electromagnetic field at radio frequency. 113

Figure 2-5. Nanoparticle heating controlled release mechanisms from liposome-nanoparticle assemblies. 118

Figure 3-1. Magnetoliposome structures. 137

Figure 3-2. Specific absorption rate $(S A R)$ of the $\mathrm{Fe}_{3} \mathrm{O}_{4}$ nanoparticles as a function of $\mathrm{RF}$ field strength ( $H f$, in legend) and nanoparticle mass fraction (in water). 143 
Figure 3-3. Cryo-TEM micrographs of the nanoparticle-liposome dispersion. 145

Figure 3-4. Melting transition determined by DPH anisotropy...................................... 146

Figure 3-5. DPH anisotropy and bilayer calculated temperature with RF heating.............. 148

Figure 4-1. Schematics of D-LNA structures loaded with GNP2 and GNP4.................... 156

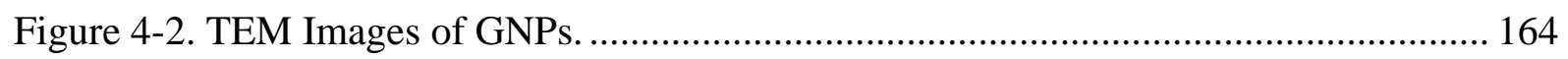

Figure 4-3. Representative Cryo-TEM micrographs. ................................................. 165

Figure 4-4. Representative Cryo-TEM and FE-SEM of GNP4 D-LNAs at Lipid:Nanoparticle

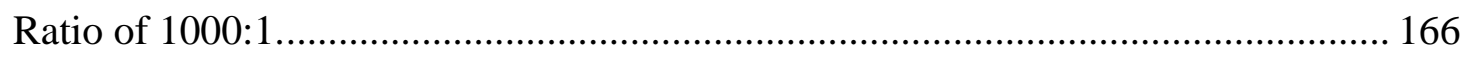

Figure 4-5. Percentage of carboxyfluorecein (CF) leakage as a function of temperature. .... 168

Figure 4-6. Change in CF leakage over change in time and excess heating capacity as a

function of temperature........................................................................... 171

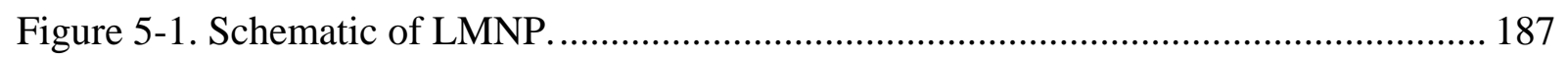

Figure 5-2. (a) LMNP Measured Hydrodynamic Diameter and $\zeta$, (b) Predicted PEG Length and Polymer Conformation, (c) Schematic and cryogenic transmission electron

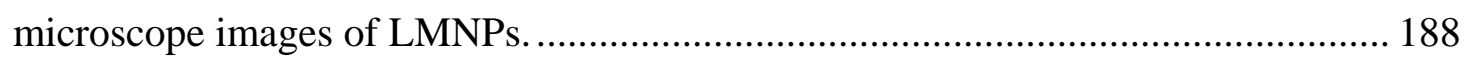

Figure 5-3. (a) Concentration of bound siRNA and $\mathrm{r}_{2}$ relaxivity and (b) percent of bound siRNA to LMNPs as a function of temperature.

Figure 5-4. (a) Change in Bulk Sample Temperature over RF Heating Time and (b) Percent

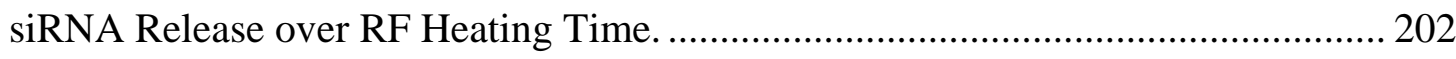




\section{CHAPTER 1}

\section{Liposome-Nanoparticle Assemblies}

Matthew R. Preiss ${ }^{1}$, Anju Gupta ${ }^{2}$, and Geoffrey D. Bothun ${ }^{1 \dagger}$

Published in: Bionanotechnology: Biological Self-Assembly and its Applications,

B.H.A. Rehm, eds. (Norfolk, UK: Caister Academic Press), pp. 265-297 (2013).

${ }^{1}$ University of Rhode Island,

Department of Chemical Engineering,

Kingston, RI, USA

${ }^{2}$ Rochester Institute of Technology,

Department of Chemical Engineering,

Rochester, NY, 14623

${ }^{\dagger}$ Correspondence: Geoffrey D Bothun

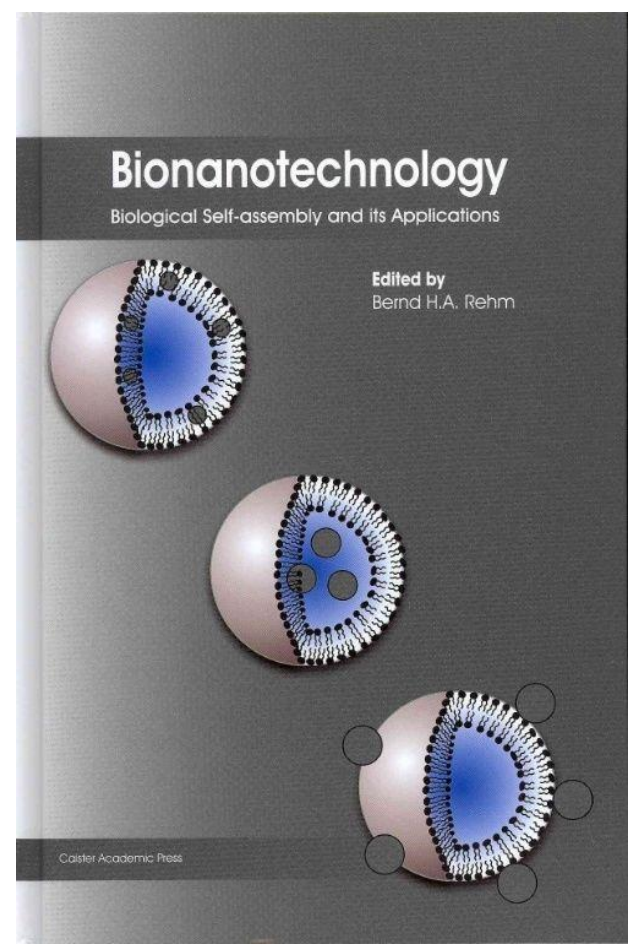

Department of Chemical Engineering

University of Rhode Island

205 Crawford Hall, 16 Greenhouse Road,

Kingston, RI, 02881, USA

Phone: +1-401-874-9518

Geoffrey D. Bothun Email: gbothun@uri.edu

Matthew R. Preiss Email: matthewpreiss@ uri.edu 


\section{Abstract}

Liposome-nanoparticle assemblies (LNAs) combine the demonstrated potential of clinically approved nanoparticles and liposomes to achieve multiple therapeutic and diagnostic objectives. Efficient and effective biomedical application requires assemblies to be stable, biocompatible, and bioavailable, while enhancing the properties of encapsulates. LNAs have been demonstrated to be very effective for in vivo and in vitro providing targeting and stimuli-responsive delivery of therapeutic and imaging agents. The ability to design LNAs with nanoparticle encapsulation, bilayerdecoration, and surface coupling provides a variety of different structures and functions. While the potential of LNAs has been demonstrated, future investigation into the interaction between the lipid bilayer and nanoparticles is necessary to understand and develop LNAs for clinical applications. This section will discuss the current state of liposome-nanoparticle assembly design, characterization, and applications of liposome-nanoparticle assemblies. 


\section{Introduction}

Only about $11 \%$ of new promising therapeutic compounds in clinical development are eventually approved. Nearly $70 \%$ of drug failures are attributed to poor pharmacokinetics, efficacy, toxicology, clinical safety, and formulation (Kola and Landis, 2004; Leeson and Davis, 2004). High drug attrition rates are the major cause of the recent decline in breakthrough drugs and the rise in costs of new drug therapies. Developments in nanotechnology have demonstrated potential for overcoming the issues related to drug pharmacokinetics and pharmacodynamics. Targeted and controlled delivery of therapeutic agents directly to targeted tissues can be achieved, improving efficacy, lowering the necessary dose, and reducing adverse effects. Nobel Laureate Paul Ehrlich's dream of a "magic bullet" to fight disease may be realized through controlled and targeted nanoscale therapeutics (Koo et al., 2005).

In 2004, the National Cancer Institute launched the Alliance for Nanotechnology in Cancer (Alliance). The Alliance's goal is development of nanotechnology-based cancer treatments and imaging. Specifically, the Alliance is emphasizing the development of drug delivery that targets tumor cells, tumor's microenvironment, and metastatic, recurrent, and drug resistant cancers with nanotherapeutic delivery systems, theranostics, contrast agents, and complexes capable of providing multiple therapies (National Cancer Institute). The design of such multifunctional constructs is inherently complex as it requires combining different molecular, colloidal, and/or particulate agents. Furthermore, the construct must be stable, resistant to protein and immune system absorption, and capable of targeting. 
Novel liposome-nanoparticle assemblies (LNAs) provide a biologically inspired route for designing multifunctional targeted therapeutics and imaging. The LNA structure is inspired by the early development of magnetoliposomes (liposomes with magnetic nanoparticles encapsulated in the aqueous core). Recent literature has referred to LNAs as "liposome-nanoparticle hybrids" (Al-Jamal and Kostarelos, 2007). LNAs are liposome structures in which nanoparticles (NPs) are encapsulated in the aqueous core, embedded in the lipid bilayer, or coupled to the bilayer surface. Liposomes are a well-established vehicle for the administration of therapeutic and diagnostic agents (Bangham and Horne, 1964; Bangham et al., 1965; Gregoriadis, 1973; Papahadjopoulos and Ohki, 1969). As a biocompatible carrier, liposomes provide a stable means for the transportation and protection of hydrophilic and/or hydrophobic molecules. Nanoparticles are nanoscale moieties that have been demonstrated to be effective transportation vehicles, contrast agents, and agents responsive to external stimuli (such as electromagnetic fields and light). LNAs combine the advantageous properties of liposomes with functional nanoparticles to create a multifunctional therapeutic and diagnostic construct (Zhang et al., 2008).

LNAs have several advantages when utilized for drug delivery, hyperthermia, imaging, and diagnostic applications. LNAs are able to delivery hydrophobic and/or hydrophilic molecules and NPs (Zhang et al., 2009). The liposome can be modified to protect encapsulated agents from biomolecule absorption and functionalized for targeting. LNAs can also be used to concentrate encapsulates, increasing the efficiency of delivery. Also, the strategies for processing, stabilizing, and targeting liposomes are 
well established (Immordino et al., 2006). NPs can be magnetically guided for in vivo targeting and provide a mechanism for stimuli-responsive triggering. Surface-bound NPs also enhance the colloidal stability of LNAs and bilayer-embedded NPs can reduce spontaneous leakage (Chen et al., 2010; Paasonen et al., 2007b; Yu et al., 2007; Zhang and Granick, 2006). LNAs harness the intrinsic advantages of a liposomal carrier, enhancing stability, bioavailability, and biocompatibility, and adds the imaging and/or responsive functionality of a NP (Zhang et al., 2008).

Drug delivering liposomes and nanoparticles have both been approved separately for clinical use by the U.S. Food and Drug Administration (FDA), see Table 1-1 and Table 1-2, respectively. These approved therapies represent the firstgeneration of development for nano-scale therapeutics and diagnostics. Combining liposomes and nanoparticles, to form multimodal LNAs, is the natural evolution for these technologies. This section will focus on a review of LNA design and structure, characterization techniques, and biomedical applications, such as controlled drug release, imaging, and hyperthermia, expanding on our group's review on stimuliresponsive LNAs (Preiss and Bothun, 2011). Recent reviews focusing on the therapeutic and diagnostic applications of liposomes are provided in references (Goyal et al., 2005; Immordino et al., 2006; Kshirsagar et al., 2005; Maurer et al., 2001; Mulder et al., 2006; Puri et al., 2009; Samad et al., 2007; Torchilin, 2005) and NPs are provided in references (Corchero and Villaverde, 2009; Emerich and Thanos, 2006; Fukumori and Ichikawa, 2006; Groneberg et al., 2006; Jin and Ye, 2007; Laurent et 
al., 2008; McCarthy and Weissleder, 2008; Michalet et al., 2005; Polyak and Friedman, 2009; Rotomskis et al., 2006; Wang et al., 2008). 
Table 1-1. FDA-approved liposomal formulations.

\begin{tabular}{|c|c|c|c|c|c|c|}
\hline Drug & Trade Name & Manufacturer & $\begin{array}{c}\text { Nano } \\
\text { Component }\end{array}$ & Treatment & $\begin{array}{c}\text { Route of } \\
\text { Administration }\end{array}$ & $\begin{array}{c}\text { FDA } \\
\text { Approval }\end{array}$ \\
\hline Amphotericin B & Abelcet & Sigma-Tau & Lipid complex & Fungal infections & Injectable & 1995 \\
\hline Amphotericin B & AmBisome & Astellas & Liposome & $\begin{array}{l}\text { Fungal and protozoal } \\
\text { infections }\end{array}$ & Injectable & 1997 \\
\hline Amphotericin B & Amphotec & $\begin{array}{l}\text { Aldopharma } \\
\text { USA }\end{array}$ & $\begin{array}{l}\text { Lipid colloidal } \\
\text { dispersion }\end{array}$ & Fungal infections & Injectable & 1996 \\
\hline Daunorubicin & DaunoXome & Galen & Liposome & $\begin{array}{l}\text { Advanced HIV Kaposi's } \\
\text { sarcoma; Ovarian cancer }\end{array}$ & Injectable & 1996 \\
\hline Cytarabine & DepoCyt & Pacira Pharms & Liposome & $\begin{array}{l}\text { Malignant lymphomatous } \\
\text { meningitis }\end{array}$ & Injectable & 1999 \\
\hline Morphine & DepoDur & \begin{tabular}{|l|} 
EKR \\
Theraputics \\
\end{tabular} & Liposome & Postsurgical analgesia & Epidural & 2004 \\
\hline Doxorubicin & Doxil & Janssen & $\begin{array}{l}\text { PEGylated } \\
\text { liposomes }\end{array}$ & $\begin{array}{l}\text { Metastatic ovarian cancer; } \\
\text { AIDS-related Kaposi's } \\
\text { sarcoma }\end{array}$ & Injectable & 1995 \\
\hline Verteporfin & Visudyne & QLT & Liposome & $\begin{array}{l}\text { Age-related macular } \\
\text { degeneration, pathologic } \\
\text { myopia, ocular } \\
\text { histoplasmosis }\end{array}$ & Injectable & 2000 \\
\hline Propofol & Diprivan & APP Pharms & Liposome & Anesthetic & Injectable & 1989 \\
\hline Bupivacaine & Exparel & $\begin{array}{l}\text { Pacira } \\
\text { Pharmaceuticals }\end{array}$ & Liposome & Analgesic & Injectable & 2011 \\
\hline
\end{tabular}


Table 1-2. FDA-approved therapeutic nanoparticles.

\begin{tabular}{|l|c|c|c|c|c|c|}
\hline \multicolumn{1}{|c|}{ Drug } & $\begin{array}{c}\text { Trade } \\
\text { Name }\end{array}$ & Manufacturer & $\begin{array}{c}\text { Nano } \\
\text { Component }\end{array}$ & Treatment & $\begin{array}{c}\text { Route of } \\
\text { Administration }\end{array}$ & $\begin{array}{c}\text { FDproval } \\
\text { Approm }\end{array}$ \\
\hline $\begin{array}{l}\text { Superparamagnetic } \\
\text { Iron Oxide }\end{array}$ & Feridex & AMAG Pharms & Nanoparticle & Liver tumor imaging & Injectable & 1996 \\
\hline $\begin{array}{l}\text { Superparamagnetic } \\
\text { Iron Oxide }\end{array}$ & Gastromark & AMAG Pharms & Nanoparticle & $\begin{array}{c}\text { Gastrointestinal } \\
\text { imaging }\end{array}$ & Oral \\
\hline $\begin{array}{l}\text { Superparamagnetic } \\
\text { Iron Oxide }\end{array}$ & Feraheme & AMAG Pharms & Nanoparticle & Iron deficiency anemia & IV \\
\hline Gold & Verigene & Nanosphere & Nanoparticle & Nucleic Acid Detection & Medical Device & 2009 \\
\hline Silver & Acticoat & $\begin{array}{c}\text { Smith \& } \\
\text { Nephew }\end{array}$ & Nanoparticle & $\begin{array}{c}\text { Anti-microbial } \\
\text { Dressing }\end{array}$ & Medical Device & 1996 \\
\hline Silver & Allevyn Ag & $\begin{array}{c}\text { Smith \& } \\
\text { Nephew }\end{array}$ & Nanoparticle & $\begin{array}{c}\text { Anti-microbial } \\
\text { Dressing }\end{array}$ & Medical Device & 2007 \\
\hline
\end{tabular}




\section{Liposome}

Since the early work by Bangham, Papahadjopoulos, and Gregoriadis in the 1960-1970s, liposomes have become one of the most highly investigated nanostructures. Today, liposomes are used as model biological membranes and for therapeutic and diagnostic agent delivery (Bangham and Horne, 1964; Bangham et al., 1965; Gregoriadis, 1973; Papahadjopoulos and Ohki, 1969). Liposomes are reliable systemic drug delivery systems because they are non-toxic, biocompatible, capable of

prolonging bioavailability of encapsulated agents by reducing or preventing drug degradation and enhancing solubility and stability (Al-Jamal and Kostarelos, 2007).

Liposomes, as depicted in Figure 1-1(A), are composed of self-assembled spherical vesicles consisting of one or multiple lipid bilayers surrounding an internal aqueous core. Bilayer thickness $\left(l_{\mathrm{b}}\right)$ is $\sim 5 \mathrm{~nm}$ thick $\left(l_{\mathrm{b}}\right)$, composed of a hydrophobic acyl lipid tail region $(\sim 3 \mathrm{~nm})$ and a hydrophilic headgroup. Liposomes can be prepared with zwitterionic, anionic, or cationic lipids, and the net liposome surface charge can be adjusted by mixing different ratios of these components. From a morphological aspect, liposomes are distinguished according to their diameter, small $(<100 \mathrm{~nm})$, large $(100-1000 \mathrm{~nm}$, or giant (>1000 nm), and number of bilayers, single (unilamellar) or multiple (multilamellar) (Sivashankar, 2011). Figure 1-1(B) is a cryogenic transmission electron microscope (cryo-TEM) image of liposomes depicting the structures that can be formed. For drug delivery and diagnostics, liposomes are attractive because of their ability to encapsulate both hydrophilic (in the aqueous core or bound to the liposome surface) and hydrophobic (in the lipid bilayer) molecules. 
Liposomes also open the therapeutic window, reducing adverse effects, by altering the pharmacokinetic and pharmacodynamic characteristics of the encapsulated agent (AlJamal and Kostarelos, 2007).
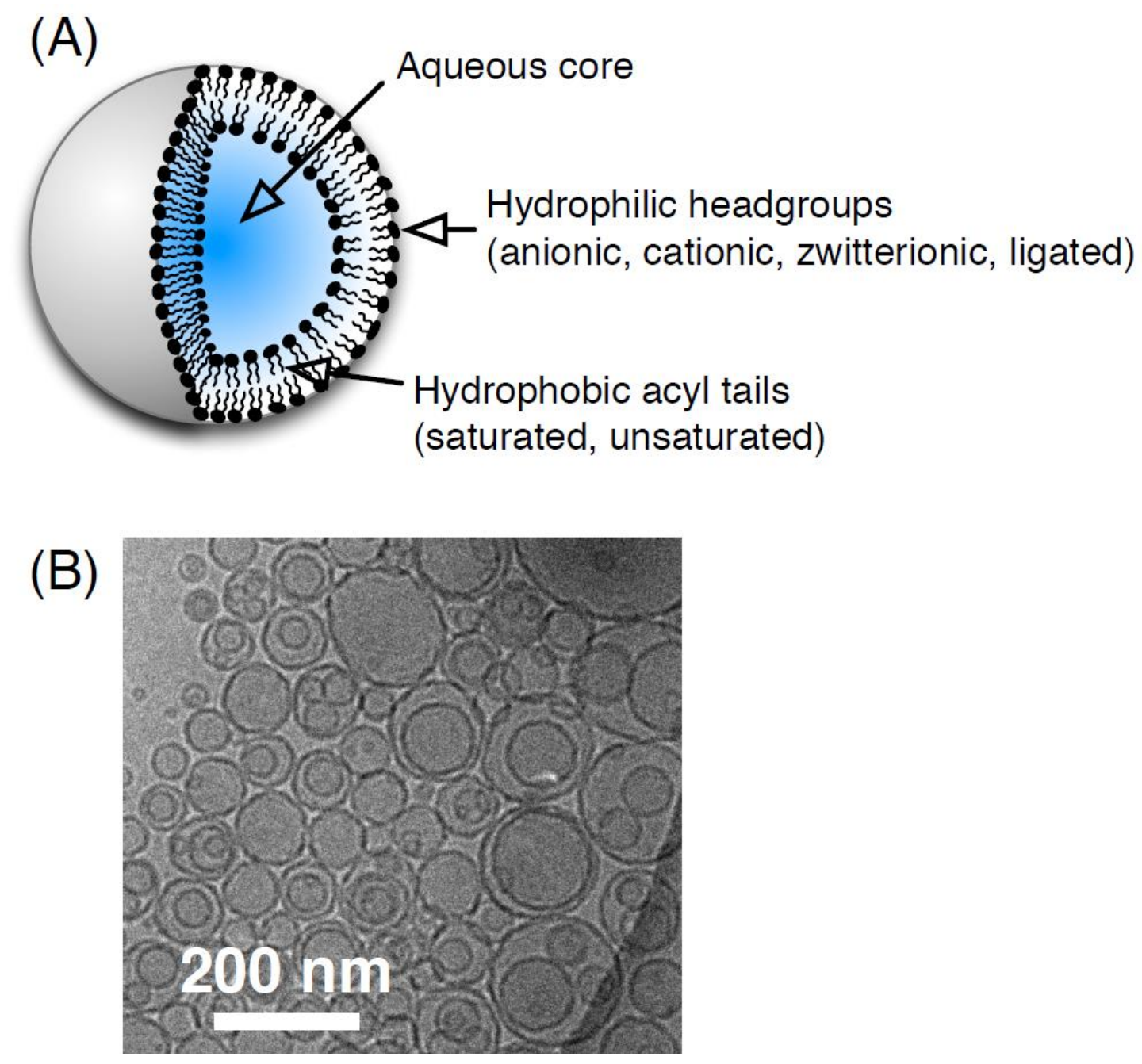

Figure 1-1. Schematic and cryogenic transmission electron micrograph of dipalmitoylphosphatidylcholine liposomes (DPPC).

(A) Liposome schematic depicting the aqueous core, hydrophilic headgroup, and hydrophobic tail regions and (B) a cryogenic transmission electron micrograph of dipalmitoylphosphatidylcholine liposomes (DPPC, $10 \mathrm{mM}$ ) prepared in phosphate buffered saline. 
FDA-approved liposomal amphotericin B formulations (Abelcet®, AmBisome ${ }^{\circledR}$, and Amphotec () are good examples of the effectiveness of liposomes for drug delivery. Amphotericin B is considered the "gold standard" for systemic treatment of fungal infections. However, amphotericin B is hydrophobic and nephrotoxic, limiting its stability and administered dosage. Encapsulation of amphotericin B in the lipid bilayer reduced the concentration of amphotericin B in the kidneys, providing similar efficacy as conventional amphotericin B while significantly reducing adverse side-effects (Gibaldi et al., 2007; Moen et al., 2009).

Release of encapsulated molecules from liposomes is controlled by the permeability through the lipid bilayer, which can be achieved by transbilayer diffusion or transient pore formation triggered by bilayer disruption or phase separation. Phase separation can be induced by 'melting' the liposomal bilayers - i.e. heating to a temperature greater than the characteristic main phase transition or melting temperature of the lipids $\left(T_{\mathrm{m}}\right)$. Below $T_{\mathrm{m}}$ the lipids are in the solid or gel phase in which the lipids are rigid and highly organized. Above $T_{\mathrm{m}}$ the lipids are disordered in a liquid crystalline or fluid phase. Permeability is high at the interface between gel and fluid phases. Phase separation and bilayer permeability can be manipulated by adjusting the lipid bilayer composition. A simple example illustrating this principle can be made with dipalmitoylphosphatidylcholine (DPPC, $\left.T_{\mathrm{m}}=42{ }^{\circ} \mathrm{C}\right)$ and dimyristoylphosphatidylcholine (DMPC, $T_{\mathrm{m}}=23^{\circ} \mathrm{C}$ ). At a DPPC/DMPC molar ratio of $74: 26$ the melting temperature occurs at physiological temperature $\left(37^{\circ} \mathrm{C}\right)$. Furthermore, cholesterol is commonly incorporated into the bilayer to reduce 
membrane fluidity above the melting temperature. Membrane fluidity has been shown to be affected by $\mathrm{pH}$, ion concentration, and the presence of molecules (such as nanoparticles) absorbed into the bilayer (Al-Jamal and Kostarelos, 2007; Bothun, 2008; Chen et al., 2010).

A major limitation to liposomal drug delivery is the short half-life (Zhang et al., 2008). Within minutes, the reticuloendothelial system (RES) will eliminate the liposomes from the blood, limiting the drug's efficacy and ability to accumulate at target sites (Moghimi and Szebeni, 2003). Proteins, called opsonins, recognize and target foreign agents (such as untargeted liposomes) for elimination by the mononuclear phagocyte system (MPS) or by hepatocyte uptake. Other proteins are capable of lysing liposomes directly by compromising the stability of the lipid bilayer (Ishida et al., 2002; Maurer et al., 2001; Yan et al., 2005). Liposome residence time is dependent on liposome size, surface charge, lipid packing, bilayer composition, and surface modifiers (Maurer et al., 2001; Samad et al., 2007). Attaching polyethylene glycol (PEG) to the liposome, forming "stealth liposomes", can increasing half-life to 2-24 hours and increase liposome stability (Medina et al., 2004; Moghimi and Szebeni, 2003; Zhang et al., 2008). The first FDA approved liposomal drug formulation (and FDA approved "nanodrug”) was Doxil ${ }^{\circledR}$ in 1995 . Doxil ${ }^{\circledR}$ is doxorubicin, the most commonly used anthracycline anticancer drug, encapsulated within a PEGylated liposome. The elimination half-life for Doxil® is 55 hours and an area under the plasma concentration time curve of $900 \mu \mathrm{g} \mathrm{h} \mathrm{mL}-1$, compared to 0.2 hours and $4 \mu \mathrm{g} \mathrm{h} \mathrm{mL} L^{-1}$ for free doxorubicin (Barenholz, 2012; Chang and Yeh, 2012). 
Drug delivery from liposomes can also be accomplished by cellular uptake, which can occur by adsorption, endocytosis, fusion, and/or lipid transfer (Pagano and Weinstein, 1978; Samad et al., 2007; Torchilin, 2005). Adsorption is the association of liposome bilayer with cell bilayer without destroying the liposome bilayer or being internalized by the cell. Adsorption can be specific (assisted by targeting ligands such as antibodies) or nonspecific (controlled by intermolecular and surface forces). Endocytosis involves the uptake of liposomes into the cell by encapsulation within endosomes. Release of drugs to the cytoplasm can occur by membrane destabilization of the encapsulating endosome or by delivery to lysosomes. Lysosomes have an acidic $\mathrm{pH}$ and contain lysing enzymes. Drug release is accomplished when lysosome enzymes hydrolyze the lipid bilayer releasing the drug. Lysosome drug release is only effective when the encapsulated drugs are not susceptible to lysosome enzymes and $\mathrm{pH}$. Fusion involves the adsorption and incorporation of the liposome bilayer with the cell membrane, releasing the payload into the cytoplasm. Finally, lipid transfer involves the exchange of lipids between the liposome bilayer and the cell membrane without enveloping the liposome (Samad et al., 2007; Torchilin, 2005).

\section{Nanoparticles}

Nanoparticles are nanoscale moieties having magnetic and optical properties for use in therapeutic and imaging applications. The high surface area-to-volume ratio, stability, functionalization, and size (1-100nm, on the order of biological macromolecules) of nanoparticles make them particularly attractive for biomedical 
applications. Nanoparticles have shown to be particularly effective as a contrast agent, a heat source, and as a targeting agent. Clinical application of nanoparticles can be hindered by poor colloidal stability, hydrophobicity, protein absorption, immune system uptake, and cytotoxicity. LNAs provide a carrier to take advantage of the properties of nanoparticles for controlled release, targeted therapies, hyperthermia, diagnostics, and imaging applications (Al-Jamal and Kostarelos, 2007; Huang et al., 2011). A number of different inorganic nanoparticles have been used in LNAs, such as quantum dots (Al-Jamal et al., 2008b; Bothun et al., 2009; Gopalakrishnan et al., 2006), fullerenes (fullerenosomes) (Babincova et al., 2003, 2004; Chen and Bothun, 2009; Doi et al., 2008; Hwang and Mauzerall, 1993; Ikeda and Kikuchi, 2008; Ikeda et al., 2009; Ikeda et al., 2005; Jeng et al., 2005; Niu and Manzerall, 1996), silver (Bothun, 2008; Park et al., 2005), superparamagnetic iron oxide (SPIO) (Bothun and Priess, 2011; Chen et al., 2010), and gold (Park et al., 2006). This section will discuss several nanoparticles that have been utilized in LNA applications.

Despite their applications in drug and gene delivery and cosmetics, cytotoxicity remains a major concern. Understanding the interactions between nanoparticles and cell membranes is crucial to NP biomedical applications and provides insight into their toxicity. NPs can be designed to bind on the cell surface, adsorb within the membrane, and translocate across the cell membrane. NPs can be exploited for novel applications by controlling the interaction between the NP and bilayer. A common way to achieve this interaction is by modifying the surface of the NP, specifically by adding positive or negative charges onto NP surface (N. Li, 2006; 
S. Legrand, 2008). Binding interaction between superparamagnetic iron oxide particles and stem cells are being used in cell selection process (L.F. Pavon, 2008). NPs used in drug delivery applications can be modified to avoid drug degradation by increasing the circulation period which in turn results in cell uptake efficiency (S. Jin, 2007).

\subsection{Quantum Dots}

Quantum dots (QDs), 2-10nm florescent semiconductor nanocrystals, have been demonstrated as effective imaging and diagnostics agents. QDs can provide a highly sensitive contrast agent capable of exhibiting fluorescence that is 10-20 times greater than conventional imaging agents, such as organic dyes and florescent proteins. QDs are also 100 times more stable against photobleaching than organic dyes (Chan, 1998). The optical properties of QDs can be tuned by adjusting their size and composition. Commonly used quantum dots for biomedical applications include cadmium selenide (CdSe), cadmium telluride (CdTe), indium phosphide (InP), and indium arsenide (InAs) (Bharali and Mousa, 2010). Clinical application of quantum dots is limited due to their inherent hydrophobicity and potential cytotoxicity. Conjugation of quantum dots with liposomes have shown to be effective to overcome these limitations (Al-Jamal and Kostarelos, 2007; Bothun et al., 2009; Dudu et al., 2008; Smith et al., 2006; Walling et al., 2009; Weng et al., 2008). 


\subsection{Gold Nanoparticles}

Imaging and photothermal effects of gold NPs stem from their enhanced surface plasmon resonance (SPR), where visible or near-infrared light is absorbed causing oscillation of surface electrons (Huang et al., 2010). SPR absorbance and the wavelength range are dependent upon nanoparticle size, core/shell configuration (e.g. silica core/gold shell (Oldenburg et al., 1999)), and geometry. Shifts in these properties are indicative of the degree of NP aggregation and/or molecular adsorption on the NP surface ( $\mathrm{Li}$ and $\mathrm{Gu}, 2010$ ). For photothermal therapy, absorbed light energy is converted into local heat that thermally diffuses into the surrounding medium. Varying NP size and core/shell configuration provides a means of tuning the frequency window for photothermal therapy. It is generally accepted that gold NPmediated phototherapy is attributed to heat or resulting bubble nucleation depending on the light intensity and mode of exposure ( $\mathrm{Li}$ and $\mathrm{Gu}, 2010)$. However, recent work by Krpetic et al. at low light energies suggests that photochemical effects - the formation of free radicals during NP irradiation - may play an important role. In addition to photothermal heating, electromagnetic fields operating at RF can be used to heat gold NPs (Krpetic et al., 2010). For example, Gannon et al. examined the effect of NP concentration and RF field strength on the heating rates of $5 \mathrm{~nm}$ Au NPs in water. A rate of $\sim 74{ }^{\circ} \mathrm{C} \mathrm{min}^{-1}$ was measured using an $800 \mathrm{~W}$ RF field at a NP concentration of $67 \mu \mathrm{M}$ (Gannon et al., 2008). 


\subsection{Superparamagnetic Iron Oxide Nanoparticles}

Superparamagnetic iron oxide (SPIO) NPs are 4-20nm nanoparticles typically composed of magnetite $\left(\mathrm{Fe}_{3} \mathrm{O}_{4}\right)$ or maghemite $\left(\gamma-\mathrm{Fe}_{2} \mathrm{O}_{3}\right)$. SPIO NPs demonstrate physical and magnetic properties, such as low toxicity and paramagnetism, making them advantageous for in vitro and in vivo applications. Due to the nanoscale crystal size of iron oxide, a single magnetic domain forms making the particle superparamagnetic. The atomic magnetic dipoles of paramagnetic materials are randomly oriented due to Brownian fluctuation in the absence of a magnetic field. Presence of a magnetic field causes the crystals to align in the direction of the field. After removal of the magnetic field, Brownian fluctuation will cause the random orientation, leaving no magnetic reminisce (Thorek et al., 2006). The superparamagnetic characteristics of SPIO NPs allow them to be used as contrast agents for magnetic resonance imaging (MRI), targeted therapeutic agents capable of being directed under a static magnetic field, and a heat source from when exposed to alternating current electromagnetic fields (AC EMF) (Brezovich, 1988; Teja and Koh, 2009). SPIO NPs also have low toxicity because the iron oxide is broken down naturally by the liver and spleen (Laurent et al., 2008; Mornet et al., 2004; Pankhurst et al., 2003; Rivera Gil et al., 2010). The characteristics of SPIO NPs allow for the development of multifunctional LNAs capable of simultaneous targeting, imaging, hyperthermia and/or drug delivery. 


\section{Formation, Structure, and Design Strategies}

The functionality of a LNA is determined by the liposome composition, liposome and NP surface modifiers, NPs employed, intermolecular and surface interactions, and colloidal stability. LNA design strategies include the encapsulation of individual or multiple NPs within the aqueous core of the liposome, embedding hydrophobic NPs in the lipid bilayer, and binding or conjugating NPs to the liposome surface (Figure 1-2). Table 1-3 contains a list of Au, iron oxide, and $\gamma$-iron oxide LNAs reported in the literature since 2008. LNAs can be used to protect NPs and encapsulated agents from the adsorption of exogenous molecules, enhancing bioavailability and reducing the need for complex surface chemistries. Concentration of NPs and therapeutic agents within the liposome can increase intracellular delivery, providing greater contrast for imaging, more efficient drug delivery, and enhanced heating capability for hyperthermia applications. Functionality can also be added by modifying the LNA bilayer with functional lipids or surface coatings for improved stability and providing targeting capability. 
(A) Encapsulated (E-LNAs)
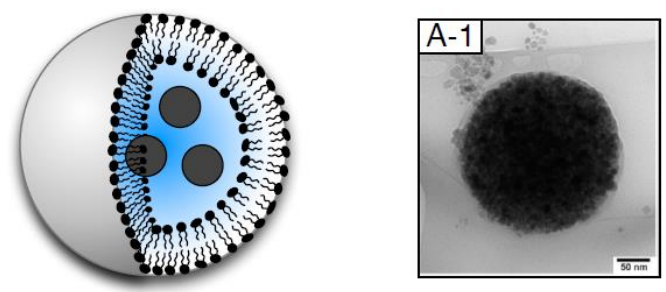

(B) Bilayer Decorated (D-LNAs)
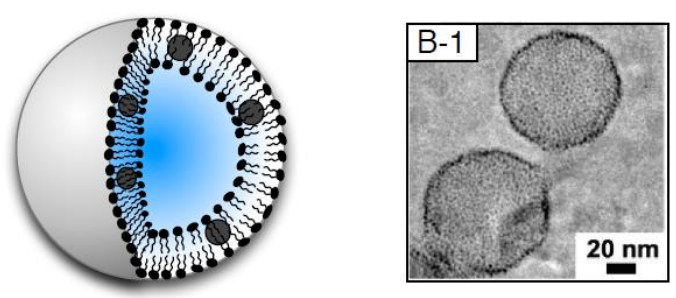

(C) Surface Coupled (S-LNAs)
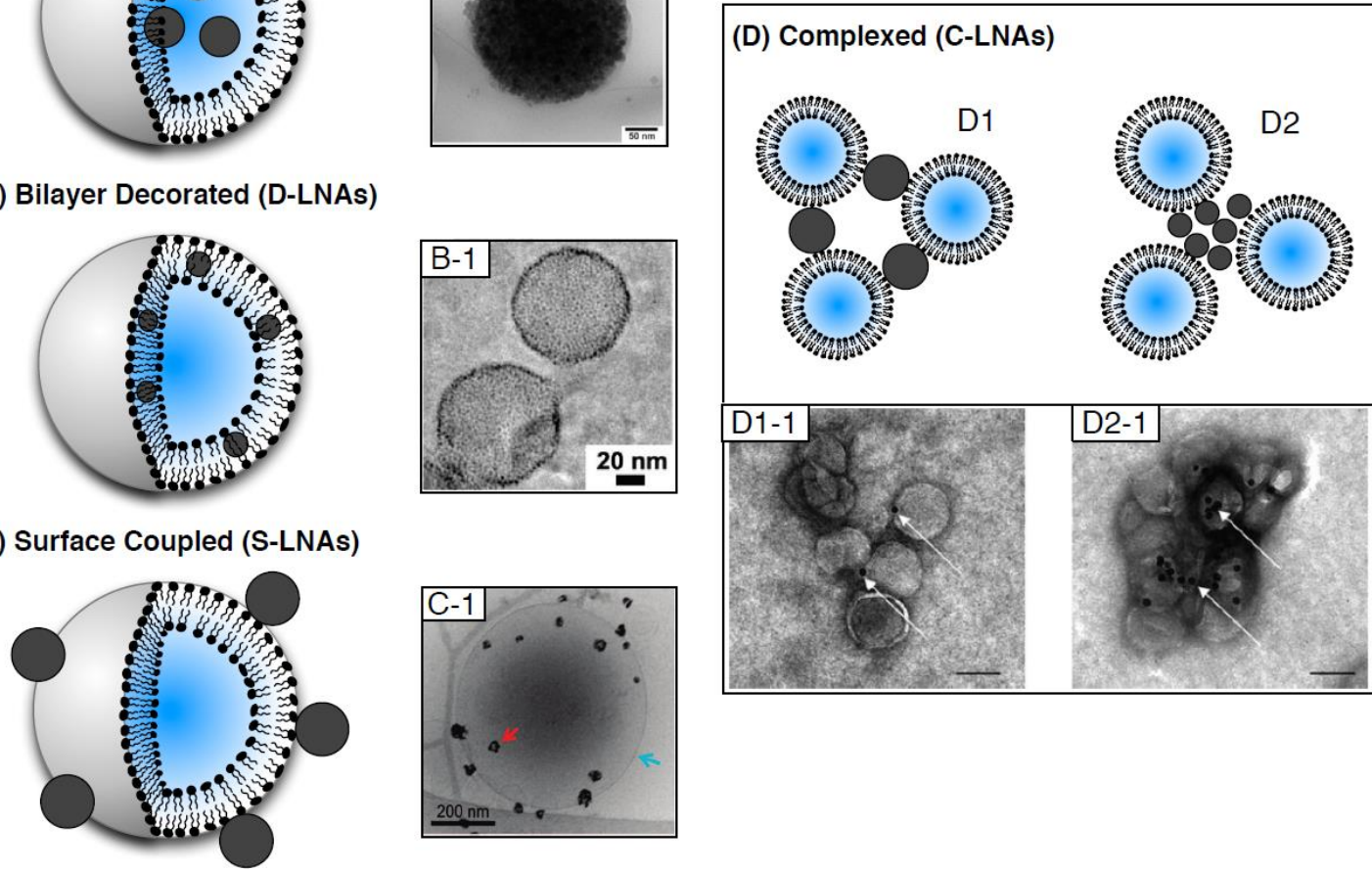

Figure 1-2. Schematics and cryogenic transmission electron micrograph of liposome-nanoparticle assemblies.

Schematic and representative transmission electron micrographs of LNAs formed by encapsulating hydrophilic nanoparticles (E-LNAs; A, A-1; Wijaya and HamadSchifferli, 2007), bilayer decorating hydrophobic nanoparticles (D-LNAs; B, B-1; Rasch et al., 2010b), or surface coupling hydrophilic nanoparticles (S-LNAs; C C-1; Wu et al., 2008). Surface coupling (C) can also be used to create controlled aggregates or complexes (D1, D2; D1-1, D2-1; Volodkin et al., 2009). Structures and proportions are not to scale. Reprinted from Rasch et al., 2010b; Volodkin et al., 2009; Wijaya and Hamad-Schifferli, 2007; Wu et al., 2008

LNA functionality extends beyond that of a traditional liposome. Liposome delivery requires not just creating a stable system capable of retaining cargo during both storage and circulation, but also the ability to release encapsulates at a target site. Efficient release can be achieved by using environmental responsive liposomes that melt near physiological temperature or through chemical mechanisms, such as $\mathrm{pH}-$ 
sensitive lipids. Controlled and triggered-release from LNAs can be achieved by taking advantage of these liposomal responsive properties and NP RF and photothermal heating capability. Multifunctional LNAs capable of targeting, imaging, hyperthermia, and/or controlled release can be constructed by combining the advantageous properties of the nanoparticles and lipids used. 
Table 1-3. Review of LNAs formed with gold (Au) nanoparticles.

\begin{tabular}{|c|c|c|c|c|c|c|}
\hline Lipids (ratio) ${ }^{a}$ & Charge & $\begin{array}{c}\text { NP } \\
\text { diameter } \\
(\mathbf{n m}) \\
\end{array}$ & NP surface coating & Lipid:NPa & LNA & Ref \\
\hline $\begin{array}{l}\text { DOPC } \\
\text { DOPC:DOPC }+(90: 10) \\
\text { DOPC:DOPP }(9: 1) \\
\end{array}$ & $\begin{array}{l}\text { Zwitterionic } \\
\text { Cationic } \\
\text { Anionic } \\
\end{array}$ & n.r. & Ascorbic acid & n.r. & S-LNA & $\begin{array}{l}\text { Sau et al., } \\
2009\end{array}$ \\
\hline DOPC:DOTAP (8:2) & Cationic & 80 & Citrate & n.r. ${ }^{f}$ & E-LNA & $\begin{array}{c}\text { Anderson et } \\
\text { al., } 2010\end{array}$ \\
\hline DPPC:Chol (55:40) & Zwitterionic & 1.4 & n.r. & $\begin{array}{c}\text { 500:1-2000:1 } \\
\text { (DPPE-AuNP:Liposome) } \\
\end{array}$ & S-LNA & $\begin{array}{l}\text { Chithrani et } \\
\text { al., } 2010\end{array}$ \\
\hline $\begin{array}{l}\text { DPPC:DPTAP:Chol (6:3:1 } \\
\text { w/w) }\end{array}$ & Cationic & 20 & n.r. & & C-LNA & $\begin{array}{l}\text { Volodkin et } \\
\text { al., } 2009\end{array}$ \\
\hline DPPC:DSPC (9:1) & Zwitterionic & 2.5 & Hexanethiol & $17.2: 1(\mathrm{w} / \mathrm{w})$ & D-LNA & $\begin{array}{l}\text { Paasonen et } \\
\text { al., 2010b }\end{array}$ \\
\hline DPPC:DSPC (9:1) & Zwitterionic & 4 & Mercaptosuccinic acid & $10: 1(\mathrm{w} / \mathrm{w})$ & E-LNA & $\begin{array}{l}\text { Paasonen et } \\
\text { al., 2010b }\end{array}$ \\
\hline DPPC:DSPC (9:1) & Zwitterionic & 1.4 & DPPE-Nanogold ${ }^{\mathrm{TM}}$ & n.r. & $\begin{array}{l}\text { E-LNA, } \\
\text { D-LNA }\end{array}$ & $\begin{array}{l}\text { Paasonen et } \\
\text { al., 2007a }\end{array}$ \\
\hline Egg PC & Cationic & 10 & Chitosan & n.r. & S-LNA & $\begin{array}{c}\text { Pornpattanan } \\
\text { angkul et al., } \\
2011 \\
\end{array}$ \\
\hline EggPC & Zwitterionic & 2 & Dodecanethiol & 100:1-1500:1 & D-LNA & $\begin{array}{c}\text { Rasch et al., } \\
2010 \mathrm{a}\end{array}$ \\
\hline EggPC:DOTAP $(9: 1 \mathrm{w} / \mathrm{w})$ & Cationic & 4 & Mercaptopropionic acid & $\geq 3.6 \times 10^{-3}: 1(\mathrm{~mol} / \mathrm{mol})$ & S-LNA & $\begin{array}{c}\text { Pornpattanan } \\
\text { angkul et al., } \\
2010 \\
\end{array}$ \\
\hline $\begin{array}{l}\text { EYPC } \\
\text { EYPC:DDAB }(9: 1) \\
\text { EYPC:PEG-DSPE }(95: 5)\end{array}$ & $\begin{array}{l}\text { Zwitterionic } \\
\text { Cationic } \\
\text { Zwitterionic }\end{array}$ & 13 & Citrate & $10: 1,1: 1(\mathrm{~mol} / \mathrm{mol})$ & S-LNA & $\begin{array}{l}\text { Kojima et al. } \\
2008 \mathrm{~b}\end{array}$ \\
\hline
\end{tabular}


Table 1-4. Review of LNAs formed with iron oxide nanoparticles.

\begin{tabular}{|c|c|c|c|c|c|c|}
\hline \multicolumn{7}{|c|}{ Iron oxide $\left(\mathrm{Fe}_{3} \mathrm{O}_{4}\right)$} \\
\hline $\begin{array}{l}\text { [maleimide]PEG- } \\
\text { DSPE:FAM-DOPE }(10: 1 \\
\text { w/w) }\end{array}$ & & $10-14$ & $\begin{array}{l}\text { Heptanioc acid, } \\
\text { acetic acid }\end{array}$ & $1: 1.8(\mathrm{w} / \mathrm{w})$ & E-LNA & $\begin{array}{l}\text { Larsen et } \\
\text { al., } 2008\end{array}$ \\
\hline $\begin{array}{l}\text { DMPC:Chol:XL }{ }^{\mathrm{e}} \\
(47.5: 47.5: 5) \\
\text { DPPC:DMPC:XL } \\
(9.5: 85.5: 5)\end{array}$ & Zwitterionic & 10 & Catechol & $\geq 8.3: 1(\mathrm{~mol} / \mathrm{w})$ & C-LNA & $\begin{array}{l}\text { Mart et al., } \\
\quad 2009\end{array}$ \\
\hline $\begin{array}{l}\text { DMPC:DMTAP:Chol:DMP } \\
\text { E-PEG }(35: 50: 10: 5)\end{array}$ & Cationic & n.r & n.r. & n.r & E-LNA & $\begin{array}{l}\text { Dandamudi } \\
\text { et al., } 2009 \\
\end{array}$ \\
\hline $\begin{array}{l}\text { DPPC:Chol:PEG- } \\
\text { DMPE:Fol-PEG-DSPE } \\
\text { (80:20:4.2:0.5) }\end{array}$ & Zwitterionic & 10 & Lauric acid & n.r. & E-LNA & $\begin{array}{l}\text { Pradhan et } \\
\text { al., } 2010\end{array}$ \\
\hline $\mathrm{PC}$ & Zwitterionic & 12.5 & n.r. & & E-LNA & $\begin{array}{c}\text { Sabate et al., } \\
2008\end{array}$ \\
\hline PC:PE (2:1) & Zwitterionic & 10 & n.r. & n.r. & E-LNA & $\begin{array}{l}\text { Kikumori et } \\
\text { al., } 2008\end{array}$ \\
\hline \multicolumn{7}{|c|}{ Iron oxide $\left(\gamma-\mathrm{Fe}_{2} \mathrm{O}_{3}\right)$} \\
\hline $\begin{array}{l}\text { DOPC:DPTAP:Chol:DPPE: } \\
\text { PEG-DMPE:Fol-PEG- } \\
\text { DSPE } \\
(47.5: 18.9: 28.5: 1: 4: 0.1 \\
\text { w/w) }\end{array}$ & Cationic & 16 & Oleic acid & n.r & E-LNA & $\begin{array}{l}\text { Bothun et } \\
\text { al., } 2011\end{array}$ \\
\hline DOPE:Chol & Cationic & 20 & Tartaric acid & $0.75-3 \mathrm{mg} / \mathrm{ml}$ & E:LNAs & $\begin{array}{l}\text { Zheng et al., } \\
2009\end{array}$ \\
\hline DOTAP:DOPE & Cationic & 10 & $\begin{array}{l}\text { (3-Aminopropyl) } \\
\text { triethoxysilane }\end{array}$ & n.r & E-LNA & $\begin{array}{l}\text { Yang et al., } \\
2008\end{array}$ \\
\hline
\end{tabular}




\begin{tabular}{|l|c|c|c|c|c|c|}
\hline DPPC & Zwitterionic & 5 & Oleic acid & 1000:1-10000:1 & D-LNA & $\begin{array}{c}\text { Chen et al., } \\
2010\end{array}$ \\
\hline $\begin{array}{l}\text { DPPC:Chol (5:1, 15:3 w/w) } \\
\text { DPPC:DSPC:Chol (10:5:3 } \\
\text { w/w) }\end{array}$ & Zwitterionic & 43 & Dextran & n.r. & E-LNA & $\begin{array}{c}\text { Tai et al., } \\
2009\end{array}$ \\
\hline DPPC:Chol (67:33) & Zwitterionic & 10 & Glutamic acid & n.r. & E-LNA & $\begin{array}{c}\text { Zhu et al., } \\
2009\end{array}$ \\
\hline DPPC:DPTAP & Cationic & 16,30 & Oleic acid & & $\begin{array}{c}\text { Chen and } \\
\text { Bothun, } \\
2011\end{array}$ \\
\hline
\end{tabular}

${ }^{a}$ Molar ratios provided unless noted otherwise, n.r. stands for not reported.

${ }^{\mathrm{b}}$ Cationic peptide lipid (Murakami et al., 1984).

${ }^{c}$ Contained within a cyclodextran cavity and embedded via fullerene exchange method (Ikeda et al., 2005).

${ }^{\mathrm{d} C}$ Cerasome (ceramic coated liposome).

eross-linking molecule (adhesive lipid).

fEstimated at 4 liposomes per NP. 


\subsection{Encapsulated Liposome-Nanoparticle Assembly}

Encapsulated liposome-nanoparticle assemblies (E-LNAs) are formed by encapsulating NPs within the aqueous core of liposomes (Figure 1-2(A)). The first investigation of LNAs was inspired by the use of liposomes as a carrier for hydrophilic drugs. E-LNAs, by encapsulating NPs in the liposome core, force NPs to cluster together at a high density. High density nanoparticle loading is advantageous to hyperthermia and drug delivery because heating and drug release can be localized preventing damage to adjacent tissues. Also, high density loading provides a strong contrast agent for biomedical imaging (Wijaya and Hamad-Schifferli, 2007).

Magnetoliposomes (MLs), liposomes encapsulating superparamagnetic NPs, are one of the simplest and first developed LNA configurations (De Cuyper and Joniau, 1988; Shinkai et al., 1996). They can be prepared by encapsulating preformed NPs in solution or by forming NPs within the liposome core, as first shown by Papahadjopoulos in 1983 (Hong et al., 1983). E-LNAs can be prepared by thin film hydration (TFH), double emulsion (DE) (Zheng et al., 1994), or reverse phase evaporation (REV) (Szoka and Papahadjopoulos, 1978). Extrusion or sonication of post-formation liposomes can be employed to control the size of E-LNAs. Supported lipid bilayers (SLBs), NPs coated with a lipid bilayer, are formed when $d_{\text {core }}=d_{\mathrm{NP}}$. ELNA formation requires the use of colloidal stable nanoparticles with a diameter $(d)$ that is smaller than the inner diameter of the aqueous liposome core, $d_{\text {core }}>d_{\mathrm{NP}}$ (Figure 1-3(A)). The maximum theoretical number of encapsulated NPs is $n \approx$ $0.74\left(V_{\text {core }} / V_{\mathrm{NP}} ; V\right.$ represents the volume of the core or NP), due to the close packing of 
spheres and $d_{\text {core }} \gg d_{\mathrm{NP}}$. Wijaya and Hamad-Schifferli demonstrated that it is possible to approach this limit, demonstrating high-density encapsulation of $\mathrm{Fe}_{3} \mathrm{O}_{4} \mathrm{NPs}\left(d_{\mathrm{NP}}=\right.$ $12.5 \mathrm{~nm}$ ) within DPPC liposomes (Figure 1-2(A-1)). With this design the available core volume for co-encapsulating aqueous drug molecules decreases within increasing NP concentration (Wijaya and Hamad-Schifferli, 2007). However, the ability for embedding hydrophobic molecules within the bilayer is unaffected by NP concentration.

The osmotic pressure differential across the lipid bilayer and the attractive or repulsive forces between the bilayer and the NPs determine the structure of E-LNAs. The elasticity of the bilayer determines how the LNA will deform in response to these forces. Attractive forces can include van der Waals, hydrophobic, and electrostatic interactions; and repulsive forces can include electrostatic, depletion, hydration, and steric interactions. The physical stability of a liposome-NP system can be determined by the Deryaguin-Landau-Verwey-Overbreek (DLVO) theory. The DLVO theory balances the opposing forces to provide a total energy of interaction between the particles. Liposome-NP systems are characterized by three types of interactions that take place, repulsion between liposome-liposome and NP-NP and attractive forces between liposome and NP. Electrostatic repulsion becomes significant when nanoparticles and liposomes approach each other and their double layers begin to interfere. Electrostatic energy curve represents the energy required to overcome the repulsion. The maximum energy corresponds to the situation when the surfaces are touching each other and is zero outside the double layer (Leckband, 2001). 
The adhering and non-adhering characteristics of nanoparticles can lead to changes in bilayer curvature, which can impact liposome size, shape, and phase homogeneity (Lipowsky and Dobereiner, 1998). Generally, this will occur when encapsulates are different from molecules present outside (e.g. sugars or proteins) liposomes.

LNAs are generally formed with small non-adhering NPs because NP adhesion to bilayers can significantly alter LNA structure and morphology. The exception to this is LNAs formed by coating a single large NP with an adsorbed or supported lipid bilayer. For non-adhering encapsulated particles, the bilayer can curve towards the larger particles. As an example of a non-adhesive system, Pradhan et al. (Pradhan et al., 2007) compared the encapsulation efficiency of $10 \mathrm{~nm} \mathrm{MnFe} 2 \mathrm{O}_{4} \mathrm{NPs}$ coated with lauric acid composed of egg-PC:cholesterol (at molar ratios of 1:0, 2:1, 3:2, 1:1, and 1:2) and formed by TFH and DE. In general, TFH resulted in higher encapsulation efficiency with smaller ML diameter compared to DE due to stripping of lauric acid during the DE process. In both cases, the observation that an Egg PC:cholesterol ratio of 2:1 yielded the best encapsulation efficiency ( $70 \%$ via TFH) was attributed to cholesterol inducing a single liquid ordered bilayer phase.

In contrast, for small adhering encapsulated particles (attractive) where $d_{\text {core }}>>$ $d_{\mathrm{NP}}$ and $d_{\mathrm{NP}}<2 l_{\mathrm{b}}$ the bilayer can curve away from the particles. For large adhering particles, where $d_{\mathrm{NP}}>>2 l$, the bilayer can curve around or engulf the particles. For example, Sabate et al. examined the effect of $\mathrm{Fe}_{3} \mathrm{O}_{4} \mathrm{NP}$ concentration coated with tetramethylammonium hydroxide (58 nm hydrodynamic $d_{\mathrm{NP}}$ ) on the encapsulation efficiency of extruded soybean PC MLs. The encapsulation efficiency decreased from 
96.6\% at $1.22 \mathrm{~g} \mathrm{Fe}_{3} \mathrm{O}_{4} / \mathrm{mol} \mathrm{PC}$ to $18.5 \%$ at $119.95 \mathrm{~g} \mathrm{Fe}_{3} \mathrm{O}_{4} / \mathrm{mol}^{\mathrm{PC}}$. This was attributed to electrostatic interactions (attraction) between the cationic NPs and the PC bilayers. The size of the MLs increased from 140 to $197 \mathrm{~nm}$, consistent with lower curvature due to NP adhesion at the inner bilayer surface (Sabate et al., 2008).

Electrolytes can also effect the curvature of lipid bilayers (Lipowsky and Dobereiner, 1998). Gomes et al. (Gomes et al., 2009) prepared polyelectrolyte-coated MLs by encapsulating $8 \mathrm{~nm}$ anionic $\gamma-\mathrm{Fe}_{2} \mathrm{O}_{3}$ NPs within egg PC liposomes and then coating with alternating poly(allylamine hydrochloride) and poly(sodium 4styrenesulfonate) layers. The final coating determined the surface charge (anionic PSS or cationic PAH). The size ranged from $200-400 \mathrm{~nm}$ and two or more polyelectrolyte coatings sufficiently protected the lipid bilayer from detergent-induced disruption. 


\begin{tabular}{|c|c|c|c|c|c|c|}
\hline Lipids (ratio) $^{\mathrm{a}}$ & Charge & $\begin{array}{l}\mathrm{D}_{\mathrm{NP}} \\
(\mathrm{nm})\end{array}$ & $\begin{array}{l}\text { NP surface } \\
\text { coating }\end{array}$ & Lipid:NPa & LNA & Ref \\
\hline \multicolumn{7}{|c|}{ Gold } \\
\hline$\overline{\text { DOPC:DOTAP }(8: 2)}$ & Cationic & 80 & Citrate & n.r. ${ }^{f}$ & E-LNA & $\begin{array}{l}\text { (Anderson et al., } \\
2010)\end{array}$ \\
\hline DPPC:DSPC (9:1) & Zwitterionic & 2.5 & Hexanethiol & $17.2: 1(\mathrm{w} / \mathrm{w})$ & D-LNA & $\begin{array}{l}\text { (Paasonen et al., } \\
\text { 2010) }\end{array}$ \\
\hline EggPC & Zwitterionic & 2 & Dodecanethiol & $100: 1-1500: 1$ & D-LNA & $\begin{array}{l}\text { (Rasch et al., } \\
2010)\end{array}$ \\
\hline DPPC:DSPC (9:1) & Zwitterionic & 4 & $\begin{array}{l}\text { Mercaptosuccinic } \\
\text { acid }\end{array}$ & $10: 1(\mathrm{w} / \mathrm{w})$ & E-LNA & $\begin{array}{l}\text { (Paasonen et al., } \\
\text { 2010) }\end{array}$ \\
\hline DPPC:DSPC (9:1) & Zwitterionic & 1.4 & DPPE-Nanogold $^{\mathrm{TM}}$ & n.r. & $\begin{array}{l}\text { E-LNA } \\
\text { D-LNA } \\
\end{array}$ & $\begin{array}{l}\text { (Paasonen et al., } \\
\text { 2007) } \\
\end{array}$ \\
\hline $\begin{array}{l}\text { DOPC } \\
\text { DOPC:DOPC+ }(90: 10) \\
\text { DOPC:DOPP }(9: 1)\end{array}$ & $\begin{array}{c}\text { Zwitterionic } \\
\text { Cationic } \\
\text { Anionic }\end{array}$ & n.r. & Ascorbic acid & n.r & S-LNA & (Sau et al., 2009) \\
\hline $\begin{array}{l}\text { EYPC } \\
\text { EYPC:DDAB }(9: 1) \\
\text { EYPC:PEG-DSPE }(95: 5)\end{array}$ & $\begin{array}{l}\text { Zwitterionic } \\
\text { Cationic } \\
\text { Zwitterionic }\end{array}$ & 13 & Citrate & $\begin{array}{c}10: 1,1: 1 \\
(\mathrm{~mol} / \mathrm{mol})\end{array}$ & S-LNA & $\begin{array}{c}\text { (Kojima et al., } \\
2008)\end{array}$ \\
\hline $\begin{array}{l}\text { EggPC:DOTAP } \\
(9: 1 \mathrm{w} / \mathrm{w})\end{array}$ & Cationic & 4 & $\begin{array}{c}\text { Mercaptopropionic } \\
\text { acid }\end{array}$ & $\begin{array}{l}\geq 3.6 \times 10^{-3}: 1 \\
(\mathrm{~mol} / \mathrm{mol})\end{array}$ & S-LNA & $\begin{array}{c}\text { (Pornpattananangk } \\
\text { ul et al., 2010) }\end{array}$ \\
\hline $\begin{array}{l}\text { DPPC:DPTAP:Chol } \\
(6: 3: 1 \mathrm{w} / \mathrm{w})\end{array}$ & Cationic & 20 & n.r. & & C-LNA & $\begin{array}{l}\text { (Volodkin et al., } \\
\text { 2009) }\end{array}$ \\
\hline
\end{tabular}




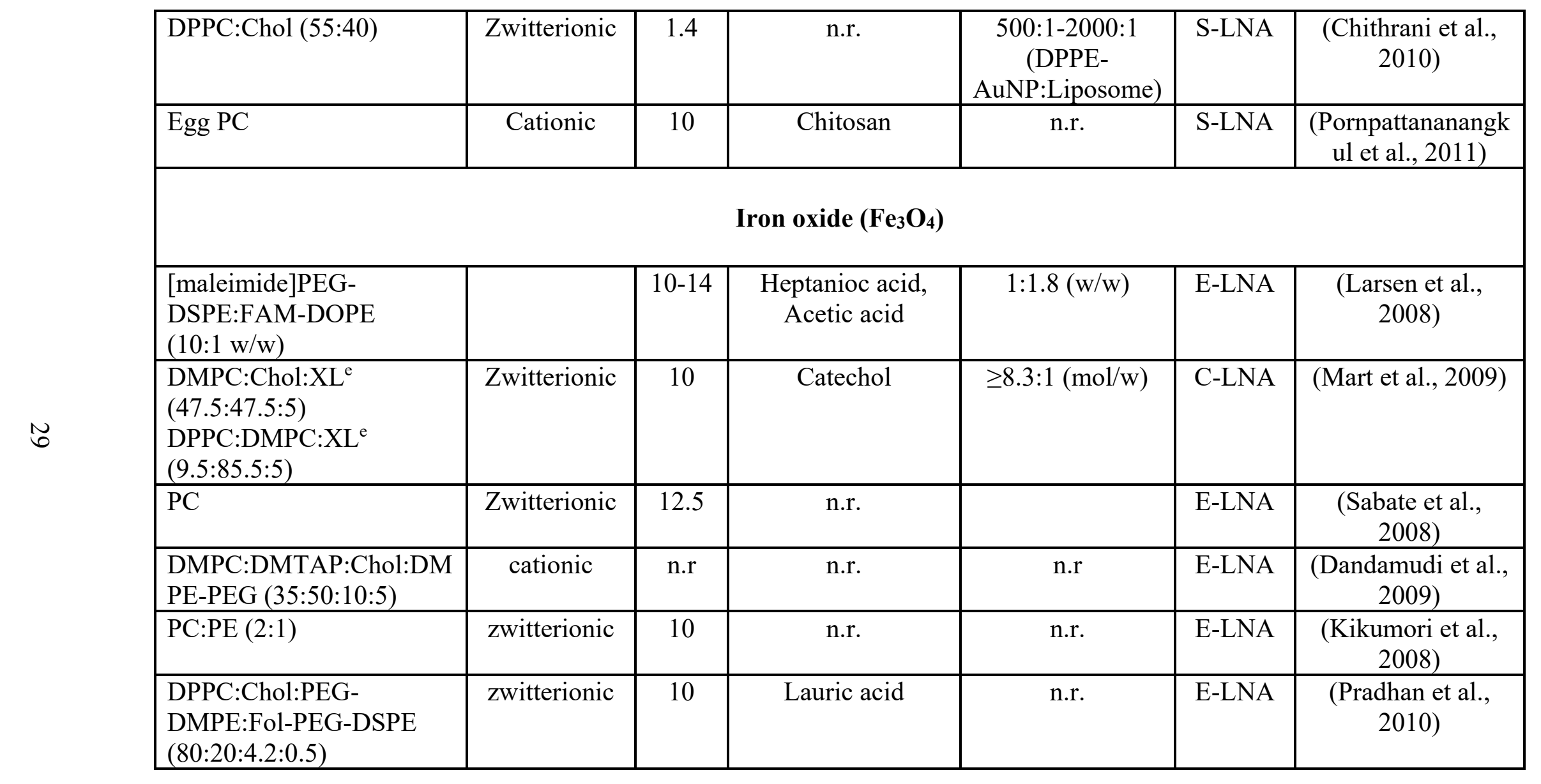




\begin{tabular}{|c|c|c|c|c|c|c|}
\hline \multicolumn{7}{|c|}{ Iron oxide $\left(\gamma-\mathrm{Fe}_{2} \mathrm{O}_{3}\right)$} \\
\hline DPPC:Chol (67:33) & zwitterionic & 10 & Glutamic acid & n.r. & E-LNA & (Zhu et al., 2009) \\
\hline $\begin{array}{l}\text { DPPC:Chol } \\
(5: 1,15: 3 \mathrm{w} / \mathrm{w}) \\
\text { DPPC:DSPC:Chol } \\
(10: 5: 3 \mathrm{w} / \mathrm{w})\end{array}$ & zwitterionic & 43 & Dextran & n.r. & E-LNA & (Tai et al., 2009) \\
\hline DPPC & zwitterionic & 5 & Oleic acid & 1000:1-10000:1 & D-LNA & (Chen et al., 2010) \\
\hline $\begin{array}{l}\text { DOPC:DPTAP:Chol:DPP } \\
\text { E:PEG-DMPE:Fol-PEG- } \\
\text { DSPE } \\
(47.5: 18.9: 28.5: 1: 4: 0.1 \\
\text { w/w) }\end{array}$ & cationic & 16 & Oleic acid & & E-LNA & $\begin{array}{c}\text { (Bothun et al., } \\
\text { 2011) }\end{array}$ \\
\hline DPPC:DPTAP & cationic & 16,30 & Oleic acid & & S-LNA & $\begin{array}{c}\text { (Chen and Bothun, } \\
\text { 2011) }\end{array}$ \\
\hline DOTAP:DOPE & cationic & 10 & $\begin{array}{c}\text { (3- } \\
\text { Aminopropyl)trieth } \\
\text { oxysilane }\end{array}$ & & E-LNA & (Yang et al., 2008) \\
\hline DOPE:Chol & catonic & 20 & Tartaric acid & $0.75-3 \mathrm{mg} / \mathrm{ml}$ & E:LNAs & $\begin{array}{c}\text { (Zheng et al., } \\
\text { 2009) }\end{array}$ \\
\hline
\end{tabular}

${ }^{a}$ molar ratios provided unless noted otherwise.

${ }^{b}$ cationic peptide lipid (Murakami et al., 1984).

${ }^{c}$ contained within a cyclodextran cavity and embedded via fullerene exchange method (Ikeda et al., 2005).

${ }^{\mathrm{d}}$ cerasome (ceramic coated liposome).

ecross-linking molecule (adhesive lipid).

festimated at 4 liposomes per NP. 


\subsection{Bilayer-Decorated Liposome-Nanoparticle Assembly}

Bilayer decorated liposome-nanoparticle assemblies (D-LNA) are liposomes with hydrophobic nanoparticles embedded in the lipid bilayer (Figure 1-2(B)). Similar to the ability of cells to accommodate membrane proteins, liposomes can distort to accommodate hydrophobic NPs that exceed the thickness of hydrophobic acyl region of the bilayer ( 3 nm) (Al-Jamal et al., 2008b; Bothun, 2008; Chen et al., 2010; Jang et al., 2003). Embedded NPs can affect lipid packing, lipid phase behavior, transbilayer permeability, and LNA structure and morphology (Binder et al., 2007; Bothun, 2008; Bothun et al., 2009; Chen et al., 2010; Chen and Bothun, 2009; Jeng et al., 2005; Park et al., 2005, 2006; Rasch et al., 2010b). Cryo-TEM can be used to observe the structure and morphology of these nano-scale systems in solution (Chen and Bothun, 2011). Atomic force microscopy has also been used to observe phaseseparated domains and monitor membrane remodeling and alteration due to the presence and distribution of nanoparticles within the bilayer (Kirat, 2010). The diameter of embedded nanoparticles (core and surface coating) is similar to the thickness of the lipid bilayer $(\sim 5 \mathrm{~nm})$. Theoretically, the diameter of embedded nanoparticle must be less than $6.5 \mathrm{~nm}$ in order for the lipid bilayer to maintain its structure. Hydrophobic nanoparticles with diameters greater than $6.5 \mathrm{~nm}$ form micelles because they are more energetically favorable due to the high local curvature strain on the bilayer, as described in Figure 1-4 (Ginzburg and Balijepalli, 2007; Wi et al., 2008). 

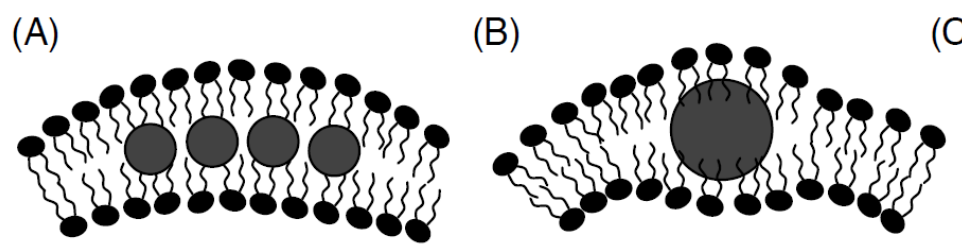

(C)

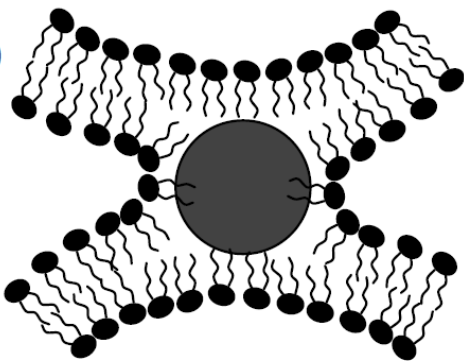

(D) $E_{\text {stretching }}\left(\sim \kappa_{\text {compressibility }}\right)$

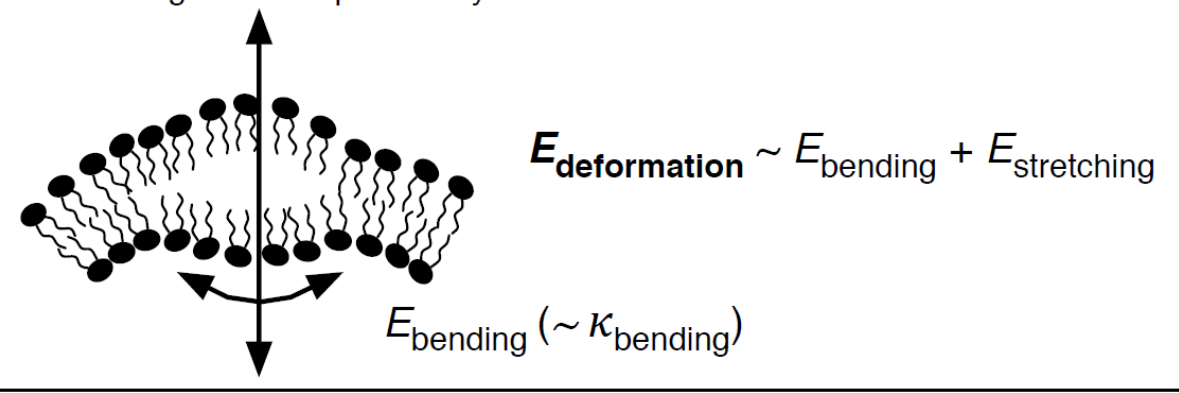

Figure 1-3. Changes in bilayer decoration mechanism of D-LNAs with increasing nanoparticle diameter.

Nanoparticle diameter, $d_{\mathrm{NP}}$, is equal to the diameter of the particle core plus two times the surface coating. (A) Small nanoparticles (defined herein as $d_{\mathrm{NP}}<2 \mathrm{~nm}$ ) can cluster together to minimize bilayer bending energy (Rasch et al., 2010b). (B) Larger nanoparticles (defined herein as $d_{\mathrm{NP}}=2-6.5 \mathrm{~nm}$ ) can create 'pockets' within the bilayer or (C) bridge adjoining liposomes (Al-Jamal et al., 2008b; Chen et al., 2010). (D) D-LNA assembly is governed in part by the bilayer deformation energy, which can be evaluated based on the energy penalty associated with lipid stretching (related to the compressibility modulus) and monolayer curvature (related to bending modulus).

The characteristics of LNAs are directly affected by embedded nanoparticles. Embedded NPs interact with the lipid acyl tails changing the fluidity of the membrane bilayer. Physical obstruction of the movement of lipid tails reduces the lipid bilayer ordering. The melting temperature of LNAs with embedded silver (Bothun, 2008), gold (Mady et al., 2011), and SPIO NPs (Chen et al., 2010) have shown to reduce the 
transition temperature of the bilayer with increased loading by fluorescence anisotropy and differential scanning calorimetry. Recent FTIR measurements of embedded gold NPs have validated the interaction of NPs and the acyl tail groups. Changes in the frequency of $\mathrm{CH}_{2}$ stretching indicated conformational change in acyl tails of bilayers with embedded NPs. Also, dynamic light scattering of citrate-stabilized gold NPs embedded in DPPC liposomes demonstrated an increase in the negative charge. Surface charge can hinder aggregation of LNAs affecting the overall stability of LNAs (Mady et al., 2011). Therefore, changes to the membrane alter LNA properties for delivery of therapeutic and diagnostic agents. The interactions between NPs and liposomes still remains misunderstood. Further investigation into the effect of nanoparticles is necessary to develop more efficient multimodal LNAs

Clustering of embedded NPs has been observed by Rasch et al. in LNAs with dodecanethiol-coated $\mathrm{Au}\left(d_{\mathrm{NP}}=1.6-1.8 \mathrm{~nm}\right)$ (Figure 1-4(A)) (Rasch et al., 2010b). They showed that high NP loading with uniform distribution can be achieved in PC liposomes via thin film hydration (with sonication and extrusion). Janus particles can be prepared with embedded NPs clustered in approximately one half of the liposomes via detergent loading followed by dialysis. Clustering occurs as the liposomes minimize the energy penalty for bilayer deformation - i.e. for a given concentration of embedded NPs the periodic bilayer bending energy needed to accommodate individual particles is greater than that needed to accommodate nanoparticle clusters. Park et al. (Park et al., 2006) and Chen et al. (Chen et al., 2010) have observed a similar clustering phenomenon with stearylamine-coated 3-4 $\mathrm{nm} \mathrm{Au}$ and oleic acid-coated 5 
$\mathrm{nm} \gamma-\mathrm{Fe}_{2} \mathrm{O}_{3}$ NPs in DPPC liposomes (Figure 2(B-1)), respectively. This suggests that $\mathrm{NP}$ clustering is not restricted to $d \mathrm{NP}<2 \mathrm{~nm}$ (Chen et al., 2010). Increasing the loading of nanoparticles in the bilayer causes turbidity to change, which can be detected by higher shifts in absorption bands in UV-vis. The shifts are attributed to stronger interactions on dipole moments on aggregate nanoparticles (Marchenko, 2010).

In addition to clustering, embedded NPs with $d_{\mathrm{NP}}=2-6.5 \mathrm{~nm}$ can reside in bilayer 'pockets' within individual (Figure 1-4(B)) or neighboring (Figure 1-4(C)) LNAs. -These cases arise when the lipid:NP ratio is high ( 1000:1 or greater). This has been observed by Al Jamal et al. (Al-Jamal et al., 2008a) for $4 \mathrm{~nm}$ hydrophobic $\mathrm{CdSe} / \mathrm{ZnS}$ core/shell quantum dots in DOPC bilayers. Embedded SPIO NPs clusters have also led to merging of adjacent liposomes. Chen et al. hypothesizes that optimal nanoparticle embedding for controlled release is a "trade-off between structural changes and aggregation, which reduce the effective liposome surface area, bilayer stabilization, which reduced bilayer permeability, and the localized heating in a EMF" (Chen et al., 2010).

\subsection{Surface-Coupled Liposome-Nanoparticle Assembly and Complexation}

Surface coupled magnetoliposomes (S-LNAs) are formed when hydrophilic NPs are absorbed onto or coupled to the outer surface of the lipid bilayer (Figure 12(C)). This is achieved through attractive surface interactions, notably long-range electrostatic attraction. An advantage of S-LNAs is the ease in which they can be prepared - adding NPs to pre-existing liposome dispersions. Similar to bilayer 
embedment, decorated bilayers also provide direct heating to the bilayer in the presence of external stimuli. The design constraint for forming S-LNAs is dependent on bilayer NP adhesion and curvature. Recent investigations have shown that NPs with $d_{\mathrm{NP}}>\sim 20 \mathrm{~nm}$ lead to the formation of SLBs due to liposome adsorption and rupture, followed by the bilayer curving around the particle (Figure 1-3(A)) (Chen and Bothun, 2011). The critical NP diameter under which S-LNAs can be formed is $d_{\mathrm{NP}}<$ $2\left(k_{\mathrm{b}} / w\right)^{1 / 2}$, where $k_{\mathrm{b}}$ is the bilayer bending elasticity, which is dependent on lipid composition and phase state, and $w$ is the adhesion energy (Roiter et al., 2008).

The Granick group has shown that stable S-LNA dispersions can be formed using zwitterionic liposomes with decorated cationic or anionic NPs $(<20 \mathrm{~nm})$ with a NP surface coverage above 25\% (Yu et al., 2007; Zhang and Granick, 2006). This was achieved by electrostatic attraction. Lower surface coverage led to aggregation, which demonstrates the need to balance the lipid:NP ratio. It was shown with isothermal titration calorimetry that upon binding the nanoparticles could restructure the lipid bilayer, inducing gel phases in fluid liposomes and fluid phases in gel liposomes (Wang et al., 2008). This observation shows that, even without external stimuli, bound NPs can induce changes in lipid phase behavior and, presumably, permeability.

NP adhesion to the outer bilayer can affect the morphology and structure of SLNAs similar to E-LNAs. Cationic nanoparticle adhesion to the outer surface of GUVs has been shown to cause pearling. The head group area of zwitterionic lipids was increased due to the use of charged particles. Attraction of the head group and 
electrostatic repulsion to the cationic nanoparticles caused a mismatch of the outer and inner curvature of liposomes. These interactions resulted in the pearling structure of the liposome (Yu and Granick, 2009).

Sau et al. have also used electrostatic binding to prepare S-LNAs with Au NPs. High NP surface coverage was achieved by using anionic Au NPs with physisorbed ascorbic acid and cationic liposomes (9:1 DOPC to ethyl-DOPC; $T_{\mathrm{m}}=-20^{\circ} \mathrm{C}$ ) (Sau et al., 2009). This high surface coverage was accompanied by NP aggregation due to the high local concentration and (likely) to charge screening via cationic lipids between bound particles (Kojima et al., 2008a). Binding was also achieved on zwitterionic and anionic liposomes with decreasing coverage (and NP aggregation), respectively. Pornpattananangkul et al. have taken this one step further and have shown that $\mathrm{pH}$ can be used to control carboxyl-modified (anionic) Au NP binding to cationic liposomes and, in turn, liposome stability. Above the pKa of the carboxyl groups the bound NPs stabilize the S-LNAs and prevent aggregation and fusion, while below the pKa the NPs detach and liposome fusion resumes (Pornpattananangkul et al., 2010).

Lastly, LNAs can be formed by complexation (C-LNAs) if the liposomes surround NP aggregates (Figure 1-2(D2)) or the NPs bind to multiple liposomes and act as "bridges" (Figure 1-2(D1)). Voldokin et al. have shown that either structure can be formed from the same anionic Au NP-cationic liposome by manipulating electrostatic interactions using via salt concentration. High $\mathrm{NaCl}$ concentration (75 $\mathrm{mM}$ ) enhanced NP aggregation (Figure 1-2(D2-1)) and low salt concentrations 
inhibited it (Figure 1-2(D1-1)). In addition to non-specific physical interactions (electrostatic), cross-linking can be used to create C-LNAs (Volodkin et al., 2009).

Mart et al. used $\mathrm{Fe}_{3} \mathrm{O}_{4}$ NPs coated with histidine groups to bind to and complex zwitterionic/cholesterol liposomes containing $\mathrm{Cu}($ iminodiacetate)functionalized lipid. The objective was to demonstrate a potential method using histidine-Cu(IDA) binding to form C-LNAs, thereby concentrating a therapeutic and an imaging agents at a target site. The resulting aggregates ranged from 20-100 $\mu \mathrm{m}$ in diameter. The C-LNAs demonstrated EMF heating and controlled release of model drugs (Mart et al., 2009).

\section{Controlled Release}

Controlled release of encapsulated payloads from LNAs can be induced by heating of nanoparticles raising temperature of the lipid bilayer. In vivo heating of magnetic nanoparticles has been demonstrated with external stimuli such as alternating current electromagnetic fields, microwaves, light irradiation, and lasers (Brazel, 2009). As discussed earlier, the bilayer permeability rises with temperature allowing release of encapsulates. Bothun and Preiss have demonstrated local bilayer heating due of $\mathrm{Fe}_{3} \mathrm{O}_{4}$ nanoparticles heated by $\mathrm{RF}$ causing phase transition. However, there was negligible difference between the bulk and local bilayer temperatures. Therefore, controlled release is likely due to both thermally-induced phase transition and mechanical rupture of the bilayer caused by NP heating. LNA controlled release with gold and iron oxide nanoparticle will be discussed herein (Bothun and Priess, 2011). 


\subsection{Gold Nanoparticles and Photothermal Effects}

Heating of Au NPs is caused by the SPR properties that convert absorbed light into heat. SPR heating makes Au NPs efficient heat sources for LNA controlled release (Sassaroli et al., 2009). Utilizing the photothermal heating of Au NPs, Paasonen et al. (Paasonen et al., 2007b) demonstrated the ability to control the release of calcein (622.6 MW) from Au LNAs composed of DPPC/DSPC at 9:1 $\left(T_{\mathrm{m}}=44.9\right.$ ${ }^{\circ} \mathrm{C}$ ) with E-, D-, and S-LNAs (Figure 1-2(A-C)). Leakage was examined with and without UV light at a wavelength of $250 \mathrm{~nm}$ over $30 \mathrm{~min}$ at $37^{\circ} \mathrm{C}$. Without UV exposure, spontaneous calcein release was observed for E-LNAs with encapsulated mercaptosuccinic acid-coated NPs and D-LNAs with embedded hexanethiol-coated NPs. This was attributed to NP-lipid interactions at the bilayer/water interface and within the acyl tail region, respectively, which reduced bilayer integrity. With UV exposure, direct contact between NPs and the liposomes via bilayer-embedment led to the greatest release ( $\sim 90 \%$ at $30 \mathrm{~min})$. Intuitively, direct contact would improve the local heat transfer from the NPs to the liposomal bilayers relative to encapsulation. This led to a gel-fluid phase transition where calcein release was presumably enhanced by diffusion at the interface between coexisting gel and fluid domains.

Volodkin et al. demonstrated the release of 5(6)-carboxyfluorescein (CF; 376.3 MW) from C-LNAs formed by the complexation of $128 \mathrm{~nm}$ cationic liposomes (DPPC/DPTAP/chol, $T_{\mathrm{m}} \sim 40-45^{\circ} \mathrm{C}$ ) and $20 \mathrm{~nm}$ anionic Au NPs. Low $\mathrm{NaCl}$ concentration (7.5 mM) yielded LNAs with NP-mediated liposome bridges (Figure 1- 
2(D1-1)) and high $\mathrm{NaCl}$ concentration (75 mM) yielded LNAs with liposome-coated NP aggregates (Figure 1-2(D-1)). CF release from the C-LNAs was observed within 5 s after near-IR irradiation (Figure 1-5) (Volodkin et al., 2009).

a)

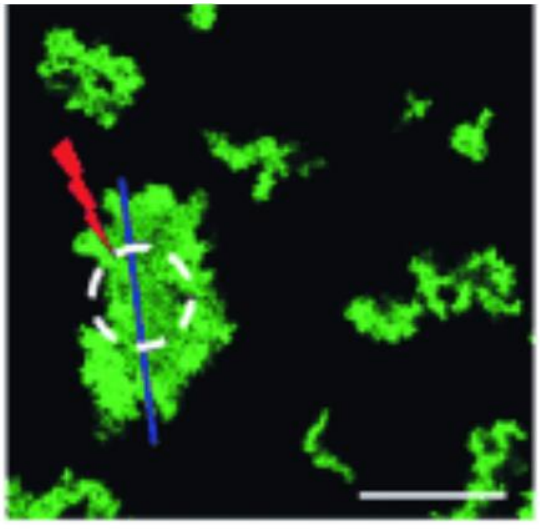

c)

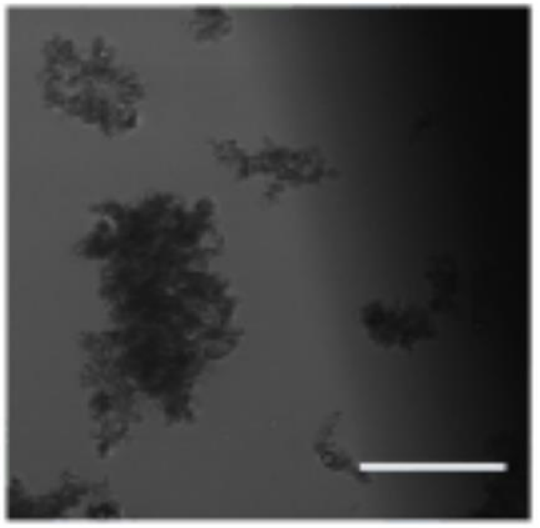

b)

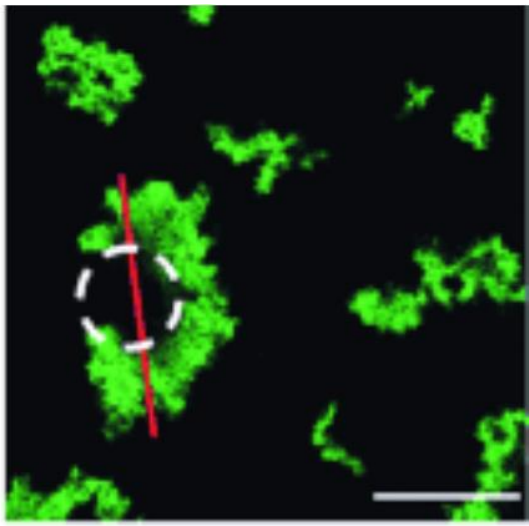

d)

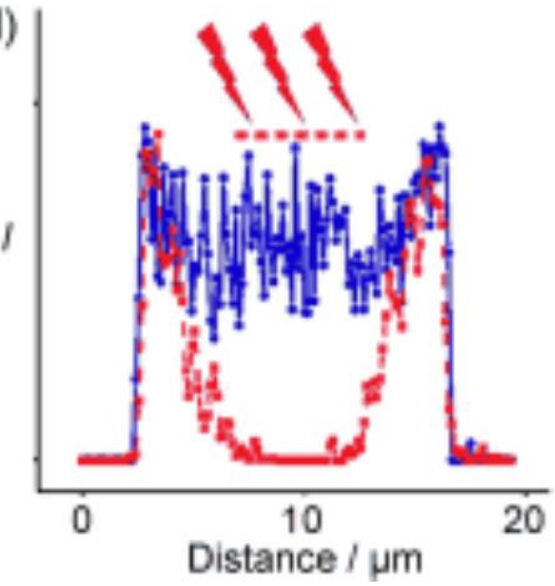

Figure 1-4. Remote dye release triggered by near IR laser illumination from CLNAs.

C-LNAs were prepared with gel-phase cationic liposomes and $20 \mathrm{~nm}$ hydrophilic gold nanoparticles. The scale bars represent $10 \mu \mathrm{m}$. These results show that near complete release can be achieved from the complexes after $5 \mathrm{~s}$ of illumination. Reprinted from (Volodkin et al., 2009) with permission.

Anderson et al. utilized the principle of plasmonic nanobubble (PNB) formation to control the release of 104 and $240 \mathrm{kDa}$ proteins from cationic LNAs ( 1 
$\mu \mathrm{m})$ containing encapsulated $80 \mathrm{~nm}$ anionic Au NPs (Figure 1-6). Irradiation was achieved using a single pump laser at $532 \mathrm{~nm}$ over $0.5 \mathrm{~ns}$. Local vapor bubble formation led to mechanical disruption, as opposed to thermal, of the LNA bilayer and rapid protein release. The advantage of PNB formation is heating is isolated within the LNA triggering the immediate release of all encapsulated cargo (Anderson et al., 2010).

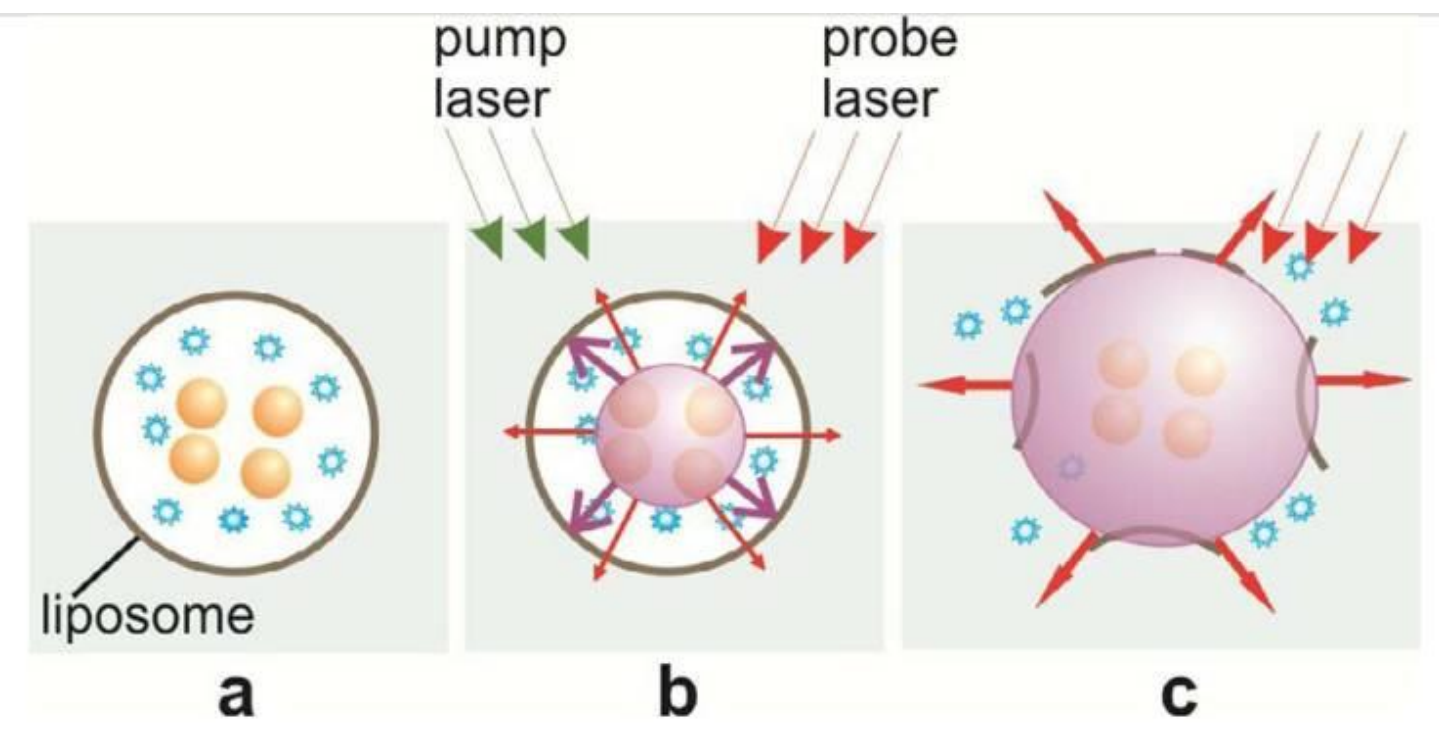

Figure 1-5. Proposed 'plasmonic nanobubble' release mechanism of encapsulated molecules from E-LNAs prepared with gold nanoparticles.

Pulsed laser irradiation of the nanoparticles nucleates vapor bubbles that expand and mechanically disrupt the liposomal bilayer and trigger permeabilization. Reprinted from (Anderson et al., 2010) with permission.

Wu et al. used hollow gold nanoshells (HGNs) encapsulated within or decorating the surface of DPPC liposomes to trigger CF release by near-IR pulses (800 $\mathrm{nm}$ ) via microbubble formation and collapse (Figure 1-2(C-1)). LNA release was dependent on the proximity of the HGNs to the liposomes (decorated or tethered 
HGNs yielded the greatest response) and the laser power. Their results strongly suggest that release was attributed to transient disruption or poration of the lipid bilayer via transient bubble cavitation (Wu et al., 2008).

\subsection{Iron Oxide Nanoparticles and Alternating Magnetic Fields}

AC EMF operating at RF heating is due to magnetic losses being converted to heat, typically at low frequencies between $100-400 \mathrm{kHz}$. The magnetic losses for NPs $<\sim 30 \mathrm{~nm}$ are due to Néel relaxation, arising from rapidly alternating magnetic dipole moments, and Brownian relaxation, arising from nanoparticle rotation and viscous losses (friction). RF heating is advantageous because it is non-invasive, easily penetrates the body, and is physiologically acceptable for up to $1 \mathrm{~h}$ if the product $H f$, where $H$ is the field amplitude (current $\times$ number of coils per length) and $f$ is the frequency, is below $4.85 \times 10^{5} \mathrm{kA} / \mathrm{m} / \mathrm{s}$ (Brezovich, 1988). NP heating capability is based on the inherent specific absorbance rate $(S A R, \mathrm{~W} / \mathrm{g})$ of the nanoparticles:

$$
S A R=\frac{c_{p}}{m_{N P}} \frac{\Delta T}{\Delta t}
$$

where $c_{p}$ is the average heat capacity of the sample, $m_{N P}$ is the NP mass, and $\Delta T / \Delta t$ is the initial heating rate of the sample. $S A R$ values up to approximately $700 \mathrm{~W} / \mathrm{g}$ can be obtained depending on the NP size, composition, and surface coating (Mornet et al., 2004). 
Tai et al. examined CF release from thermosensitive zwitterionic liposomes containing encapsulated dextran-coated $43 \mathrm{~nm} \gamma-\mathrm{Fe}_{2} \mathrm{O}_{3} \mathrm{NPs}$ (Resovist ${ }^{\mathrm{TM}}$ ) using a high frequency generator $(6.4 \mathrm{~kW}, 750-1150 \mathrm{kHz})$ operating for 5-25 min. CF release from DPPC:Chol (5:1) liposomes without encapsulated NPs was initiated between $35^{\circ} \mathrm{C}$ and $37{ }^{\circ} \mathrm{C}$. In contrast, the LNA analogs exhibited initial release at $34{ }^{\circ} \mathrm{C}$ and $32{ }^{\circ} \mathrm{C}$ with 7 and $14 \mathrm{mg} \mathrm{Fe} / \mathrm{ml}$, respectively. This initial release temperature was further tuned by increasing the cholesterol content (DPPC:Chol at 15:3) and incorporating a higher melting lipid (DSPC, $T_{\mathrm{m}}=55^{\circ} \mathrm{C}$ ). Release was attributed to NP heating. Using a rat model, they demonstrated that release could also be achieved in vivo (Tai et al., 2009).

Chen et al. have recently examined the release of CF from LNAs formed with DPPC and bilayer-embedded oleic acid-coated $5 \mathrm{~nm} \gamma-\mathrm{Fe}_{2} \mathrm{O}_{3} \mathrm{NPs}$ at lipid:NP ratios of 10000:1, 5000:1, and 1000:1 as a function of RF energy ( $1 \mathrm{~kW}$; 50-250 A, $281 \mathrm{kHz})$. Experiments were conducted at non-invasive RF energies up to 40 min and the greatest release rate was observed at 5000:1, indicating an optimal NP loading for triggering release. This optimum reflected a balance between NP loading and LNA structure - high loading is needed for triggering bilayer release, but can lead to NP aggregation and can compromise LNA structure and stability. A unique observation was the fact that increasing NP loading reduced or eliminated spontaneous leakage by increasing bilayer stability. CF release was attributed to bilayer disruption via local heating and/or LNA rupture, which produced transient voids or pores. Although, these 
findings demonstrated that higher nanoparticle embedding does not strictly equate to greater release controlled release during EMF heating (Chen et al., 2010).

\section{Targeted Therapy}

Optimal drug delivery and biomedical imaging involves the distribution of drug and/or imaging agent to the diseased tissue while minimizing adverse side-effects to healthy tissues. Adverse side-effects limit the drug dosage that may be used during treatment, potentially requiring the dosage to be reduced, delayed, and/or discontinued. Targeting LNAs limit adverse side-effects to healthy tissues and enhance drug delivery and uptake by localizing drug delivery to specific target sites (Malam et al., 2009; Minko et al., 2006).

Effective therapeutic and diagnostic treatment requires sufficient bioavailability of LNAs to diseased cells. A major limitation of LNAs, and liposomal drug delivery in general, is that the residence time is not long enough to enable the non-modified liposome assemblies to accumulate at tumor sites (Zhang et al., 2008). LNA residence time is directly related to size and surface properties (discussed herein) (Maurer et al., 2001; Samad et al., 2007).

\subsection{Passive Targeting}

Passive targeting of lipid-nanoparticle assemblies is facilitated by the enhanced permeability and retention (EPR) effect. Tumor growth requires a sufficient supply of oxygen and nutrients. Therefore, during angiogenesis, tumors will produce an increased amount of vascular endothelial growth factors routing new blood vessels 
and/or existing blood vessels directly to growing tumors. These tumor-associated neovasculatures are typically disorganized and highly permeable allowing leakage of larger entities, such as lipid-nanoparticle assemblies, into the tumor. The entities are retained by the tumor because the defective lymphatic drainage prevents larger entities from returning to the circulatory system. LNAs accumulate in the tumor providing an effective targeting mechanism that can be utilized for drug delivery, imaging, and/or hyperthermia (Cho et al., 2008; Corchero and Villaverde, 2009; van Vlerken and Amiji, 2006).

In order for LNAs to accumulate within a tumor by the EPR effect, the residence time of LNAs in the blood must be sufficient to permeate into the tumor. Longer circulating assemblies provide a greater opportunity to reach the tumor vascular system and enter the tumor for drug delivery and/or imaging. Nanoparticle elimination is primarily based upon the reticuloendothelial system (RES) uptake. LNAs (as mentioned earlier) can increase their blood residence times by reducing clearance and absorption through PEGylation.

Passive targeting also exploits the unique environment created by tumors. As stated above, rapidly proliferating tumor cells require sufficient oxygen and nutrients. However, the supply of oxygen and nutrients is typically insufficient to maintain the rapid cell growth, forcing tumor cells to breakdown sugars through glycolysis (Cho et al., 2008; Corchero and Villaverde, 2009; Minko et al., 2006; van Vlerken and Amiji, 2006). Glycolysis, under anaerobic conditions, leads to lactic acid build-up creating an 
acidic environment (Pelicano et al., 2006). LNAs comprised of pH-responsive lipids facilitating drug release in the acidic tumor environment (Cho et al., 2008).

Passive targeting can also be utilized for antibacterial applications.

Pornpattananangkul et al. (Pornpattananangkul et al., 2011) attached chitosan-coated gold to vancomycin encapsulated Egg PC liposomes. The attached gold nanoparticles prevented fusing and spontaneous leakage increasing stability and shelf-life.

Antibiotic release was triggered by the presence of bacterial toxins that caused pore formation. After 24 hours of exposure, $100 \%$ of vancomycin was released from the liposome. Vancomycin is used as a "last resort" for MRSA infections (Crossley, 2010). Such regulated LNA delivery systems reduce side effects and dosage by being capable of releasing encapsulates at the delivery site and in response to the severity of the infection.

Overall LNA size between $50-150 \mathrm{~nm}$ is optimal in order to avoid renal elimination (nanoparticles smaller than $6 \mathrm{~nm}$ ) and prevent uptake by the reticuloendothelial system (nanoparticles larger than $200 \mathrm{~nm}$ ) (Gullotti and Yeo, 2009; Veiseh et al., 2010). LNA residence time is also dependent on surface charge. Cationic LNAs demonstrate a much higher affinity to bind to the vasculature of tumors than to normal healthy tissues. Tumor vessel area targeted by cationic LNAs was approximately 5 times greater than the vessels of normal healthy tissues (Campbell et al., 2002). Cationic LNA uptake has been shown to be almost10 times higher than similar anionic and neutral LNAs (Ito et al., 2005). The preferential binding can be exploited for targeted hyperthermia, drug delivery, and/or imaging. 
Anionic and neutral entities and bodies larger than $200 \mathrm{~nm}$, upon entering the blood stream, tend to absorb antibodies, called opsonins, activating the reticuloendothelial system and inducing mononuclear phagocyte system (MPS) or by hepatocyte uptake and elimination (Ishida et al., 2002; Maurer et al., 2001; Yan et al., 2005). Incorporation of polyethylene glycol (PEG), a neutral hydrophilic polyether, onto the surface of LNAs is very effective for preventing opsonization and increasing the residence time. PEG has proven to be one of the most effective surface modifications and is probably the most used for producing long-circulating entities. For example, Doxil and Myocet in Table 1-1 is a PEG-liposome encapsulated formulation of doxorubicin. Myocet and Doxin have an elimination half-life of $2.5 \mathrm{~h}$ and $55 \mathrm{~h}$, respectively, whereas free doxorubicin has elimination half-life of $0.2 \mathrm{~h}$ (Danhier et al., 2010). Other surface modifiers and ligands, such as polysaccharides, dysopsonins, dextran, etc., have been incorporated into the bilayer to increase liposome stability, residence time, and enabling targeting capability (Zhang et al., 2008).

The final passive targeting method is topical delivery. Topical delivery is localized drug administration directly to a tumor by injection or surgery. This approach bypasses drugs administration through the circulatory system. By avoiding the circulatory system, adverse side-effects can be limited to the administration site (Minko et al., 2006). 


\subsection{Active Targeting}

Active targeting involves incorporating a targeting ligand, most often antibodies, antibody fragments, vitamins, glycolipids, or peptides (Jørgensen and Nielsen, 2009), onto the surface of LNAs. In order to be effective, the targeting ligand must specifically bind to a receptor that is exclusive to tumors or is overexpressed by tumors compared to normal healthy tissue. Active targeting can be split into two categories: cancer cell targeting and tumoral endothelium targeting. Cancer cell targeting promotes tumor internalization of LNAs and intracellular delivery of therapeutic agents. The most common receptors for cancer cell targeting are transferrin, folate receptor, cell surface glycoproteins, and the epidermal growth factor. Tumoral endothelium targeting restricts the blood supply available to tumor cells by attacking angiogenic blood vessels. The most common entities subject to tumoral endothelium targeting are vascular endothelial growth factors, vascular endothelial growth factors receptors (VEGFR-1 and VEGFR-1), $\alpha_{\mathrm{v}} \beta_{3}$ integrin, vascular cell adhesion molecule-1, and matrix metalloproteinases (Danhier et al., 2010).

Active targeting can also be accomplished with magnetic drug targeting (MDT). MDT utilizes a static magnetic field to concentration of LNAs at selected delivery sites. LNAs with encapsulated or embedded magnetic or superparamagnetic NPs can be forced to aggregate at sites with applied magnetic fields. MDT allows LNAs to be localized at an identified disease site for hyperthermia and drug delivery, minimizing the effect to adjacent tissues. 


\subsubsection{Ligand Targeting}

Active targeting of tumors by LNAs has been accomplished by targeting folate receptor (FR). Tumor cells express FRs almost 100 times normal cells. Also, the FR expression rate is proportional to the how advanced the cancer is. Therefore, FR targeting can be an effective method for identifying and treating tumors ( $\mathrm{Lu}$ and Low, 2002). FR targeting has been demonstrated both in vitro and in vivo. Folate targeted liposomes have been used with nanoparticles for tumor imaging, drug delivery, and hyperthermia (Kamaly et al., 2009; Pradhan et al., 2010).

FR targeted MLs have demonstrated selective drug delivery in vitro. PEGylated MLs incorporating FR targeting-lipids were constructed with coencapsulated doxorubicin and maghemite. Human cervical cancer cell (HeLa), which overexpress FR, bound to the cell surface and uptake from HeLa cells was observed, whereas the control showed no surface binding or uptake. FR targeting was combined with AC EMF at RF nanoparticle heating to induce doxorubicin release. Doxorubicin release under EMF treatment for $2 \mathrm{~h}$ was three times greater than without EMF (Bothun et al., 2011).

Pradhan et al. developed multifunctional thermosensitive PEGylated magnetoliposomes with a folic acid ligand capable of binding to FR. The magnetoliposomes were encapsulated with doxorubicin and iron oxide nanoparticles (Figure 1-7). Folate receptor mediated uptake of magnetoliposomes into tumor cells was demonstrated. In vitro hyperthermia and controlled release of encapsulated doxorubicin was achieved by subjecting cell lines to EMF (Pradhan et al., 2010). 


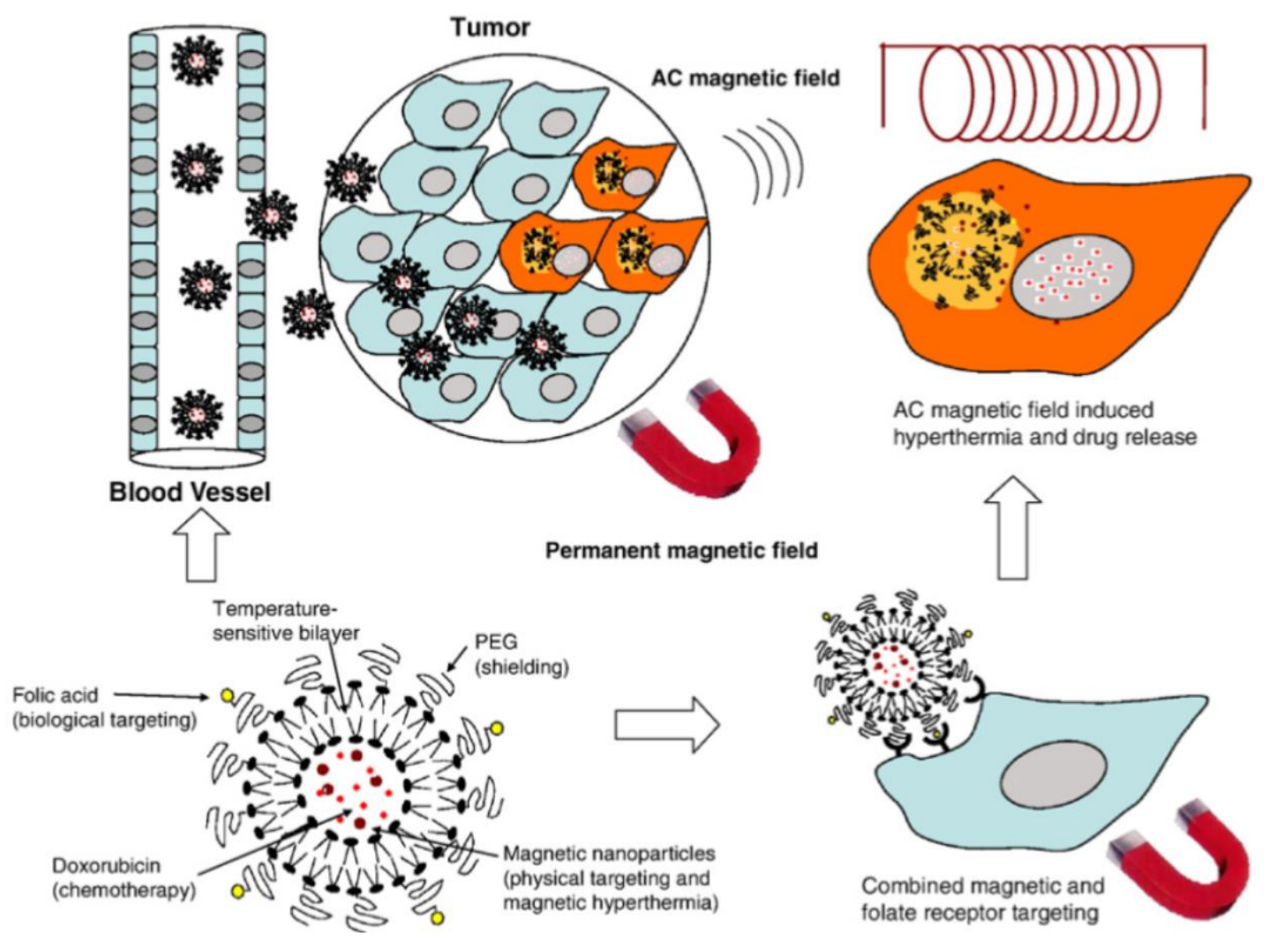

Figure 1-6. A multifunctional E-LNA for cancer thermo-chemotherapy from Pradhan et al.

In this example the LNA is a temperature sensitive magnetoliposome containing coencapsulated iron oxide nanoparticles and doxorubicin. Passive targeting can be achieved through the enhanced permeation and retention (EPR) effect of tumor vasculature, and active targeting can be achieved via folate receptor and by applying a permanent magnetic field. The application of an AC electromagnetic field can be used to release the drug and achieve hyperthermia treatment. Reprinted from (Pradhan et al., 2010) with permission.

Liposomes with incorporated antibodies or antibody fragments are referred to as immunoliposomes. A number of studies have utilized immunoliposomes and nanoparticles for biomedical applications. Antibody conjugated LNAs has been employed to target cells that overexpress epidermal growth factor receptor 2 (Her-2). Overexpression of Her-2 frequently occurs in cervical, colon, breast, prostate, brain, 
bladder, and lung cancers because it allows cells to grow more rapidly. Current cancer treatments tend to lack specificity administered causing adjacent tissues to be damaged. Her-2 targeted treatment may encounter similar adverse effects to adjacent tissues because Her-2 is a naturally occurring protein. Kullberg et al. developed a twocomponent drug delivery system to ensure that treatment would be concentrated to cells that overexpress Her-2. The two-component system requires more than one LNA to bind to a tumor cell in order to release the encapsulated drugs. The two-component system could also have two or more sets of LNAs that encapsulate different substances that are only effective when they are both present. The specificity of the twocomponent system is equal to the difference of tumor and healthy tissue Her-2 expression squared. Therefore, the two-component system could potentially deliver 3600 times more drugs to glioblastomas (capable of a 60 -fold overexpression) than normal cells. The two-fold system could hypothetically be expanded to three or more components increasing the specificity exponentially (Kullberg et al., 2005, 2009). Her2 antibodies have also been utilized to construct targeted magnetoliposomes for hyperthermia treatment. The magnetoliposomes demonstrated localized hyperthermia to Her-2 overexpressing tumors under AMF (Kikumori et al., 2008).

Cationic MLs incorporating transferrin receptor targeting antibodies (antitransferrin receptor single-chain antibody fragment or TfRscFv) have been developed by Yang et al.. SPIO NPs were encapsulated into DOTAP-DOPE liposomes with TfRscFv incorporated onto the bilayer surface. In vitro breast and pancreatic uptake of SPIO liposomes with TfRscFv antibodies was at least $\sim 2.5$ times and 11 times greater 
than non-targeting SPIO liposomes and free SPIO NPs, respectively (Yang et al., 2008).

\subsubsection{Magnetic Drug Targeting}

Accumulation with MDT is dependent on the properties of the encapsulated magnetic nanoparticles, tumor depth, blood flow, vasculature, drug binding, and concentration. MDT is also highly dependent on the external magnetic field. Penetration depth of magnets has been shown to be on the millimeter scale making MDT only effective for superficial tissues. Although, neodymium iron boron magnets have demonstrated magnetic field depths of up to $15 \mathrm{~cm}$ on SPIOs (Neuberger et al., 2005). Interest in MDT has declined in recent years due to lack of effectiveness and issues such as embolization (Sun et al., 2008).

The feasibility of magnetic targeting of with magnetoliposomes in vitro and in vivo with external magnet has been demonstrated (Fortin-Ripoche et al., 2006). MLs (E-LNAs) encapsulating $7.5 \mathrm{~nm}$ maghemite have been used as effective MRI contrast agents in vivo. MDT delivery of MLs had a MRI negative enhancement of $-52 \%$ compared to $-7 \%$ for without MDT. Enhancement was maximized after 3 hours and after 24 hours maghemite only remained in the targeted tumor (Fortin-Ripoche et al., 2006). Rivière et al. also demonstrated that real-time in vivo imaging and drug delivery of LNAs with a neodymium magnet to the brain vasculature of mice was achievable (Riviere et al., 2007). 
Magnetic targeting has also been applied to gene delivery. Transfection of pDNA was shown to be greater under the influence of a static magnetic field. After 10 min of magnetic induction, was 2.8 and 1.9 times greater for MLs (E-LNAs) with 3.0 $\mathrm{mg} / \mathrm{ml}$ and $1.5 \mathrm{mg} / \mathrm{ml}$ magnetite concentration, respectively. Also, transfection at 0.75 $\mathrm{mg} / \mathrm{ml}$ magnetite concentration was 2.6 fold greater with $30 \mathrm{~min}$ of magnetic induction than without (Zheng et al., 2009).

Dandamudi et al. utilized MDT to deliver vinblastine-loaded MLs. After application of an external magnet for $2 \mathrm{~h}$, PEGylated cationic liposomes encapsulating magnetite showed enhanced delivery of vinblastine (a chemotherapeutic drug). Tumor uptake with the magnet was $\sim 16 \%$ as compared to $\sim 5 \%$ with no magnet. MDT of vinblastine showed a significant showed an antitumor effect versus the control, reducing tumor nodules and hindering the growth of the tumor vasculature (Dandamudi et al., 2009).

Some studies incorporated MDT with other targeting techniques. Pradhan et al. examined the effect of MDT on human epidermoid carcinoma (KB) and human cervical carcinoma (HeLa) cell uptake of doxorubicin encapsulated liposomes with and without folate receptor targeting (Figure 1-7). Uptake was 2-fold higher for KB and 4-fold higher for HeLa with MDT than without. Also, the combination of MDT and folate receptor targeting uptake was 8 -fold greater in $\mathrm{KB}$ and 42 -fold greater in HeLa than without either active targeting techniques (Pradhan et al., 2010). These results suggest that further research into MDT as for supplementary targeting may be beneficial. 


\section{Diagnostics and Imaging}

Magnetic resonance imaging is a noninvasive imaging technique that can be enhanced by the use of LNAs with superparamagnetic nanoparticles, such as iron oxide. These particles are capable of being manipulated by magnetic fields allowing them to be used as a contrast agent form magnetic resonance imaging (MRI). MRI is based upon the nuclear magnetic resonance of hydrogen protons of water in the body. A strong magnetic field $\left(\mathrm{B}_{0}\right)$ is applied to the body causing these hydrogen protons align with the magnetic field. The body is then exposed to a radio frequency (RF) pulse, transverse to $\mathrm{B}_{0}$, perturbing proton alignment with $\mathrm{B}_{0}$. Relaxation, or the realignment of protons with $\mathrm{B}_{0}$ after the $\mathrm{RF}$ pulse, releases energy absorbed during the RF pulse. Two separate relaxations are monitored; longitudinal relaxation, or $\mathrm{T}_{1^{-}}$ recovery, and transverse relaxation, or $\mathrm{T}_{2}$-decay. $\mathrm{T}_{1}$-recovery (also referred to as "spin-lattice" relaxation) is the release of energy to adjacent tissue as hydrogen realigns with $\mathrm{B}_{0}$. $\mathrm{T}_{2}$-decay (also referred to as "spin-spin" relaxation) is the energy released from the exchange of energy between separate protons as nuclei goes from spinning in phase with each other (due to the RF pulse) to out of phase. 2D Fourier transform is applied to the relaxation data to generate an image. Differences in density and physical properties of tissues are related to the relaxation time and therefore can be distinguished in MRI images. Magnetic nanoparticles and LNAs can shorten the relaxation times of surrounding tissue and therefore be used as a contrast agent. A compound's effect on the relaxation of surrounding tissues, and therein its effectiveness on contrast agent, is called relaxivity. Relaxivity is defined as $R_{1}=1 / T_{1}$ 
and $\mathrm{R}_{2}=1 / \mathrm{T}_{2}$ (Mornet et al., 2004; Sun et al., 2008). Enhancement of relaxivity by LNA contrast agents is based on the concentration and inherent relaxivity of nanoparticles delivered (Fattahi et al., 2011).

MRI has also provided to be a very useful tool for observing and understanding interstitial drug delivery. Ponce et al. measured doxorubicin concentration by measuring the change in $\mathrm{T}_{1}$ relaxation of the co-encapsulated contrast agent. Spatial and temporal distribution profiles of doxorubicin and manganese from temperaturesensitive liposomes during hyperthermia is crucial to understanding tumor-drug interaction (Ponce et al., 2007). Similar real time observations will provide pharmacodynamic and pharmacokinetic information to optimize drug efficacy from LNAs.

QDs have also been utilized for to measure and observe the cellular delivery. Bothun et al. demonstrated the ability to deliver different types of QDs. Hydrophobic and hydrophilic QDs were co-encapsulated in the bilayer and aqueous core of a cationic liposome. Uptake by human hepatocellular carcinoma cells demonstrated multi-fluorescent imaging (Bothun et al., 2009). Subsequent work by Al-Jamal et al. demonstrated the release characteristics of D-LNAs containing QDs (for imaging) and encapsulated doxorubicin from gel (DSPC-QD) and fluid phase (EPC-QD) LNA bilayers. Their results showed that fluid phase D-LNAs were able to achieve $80 \%$ release over $6 \mathrm{~h}$ and that the cytotoxicity of doxorubicin was reduced through D-LNA encapsulation. The QD D-LNAs without doxorubicin exhibited minimal cytotoxicity (Figure 1-8) (Al-Jamal and Kostarelos, 2011; Tian et al., 2011). 

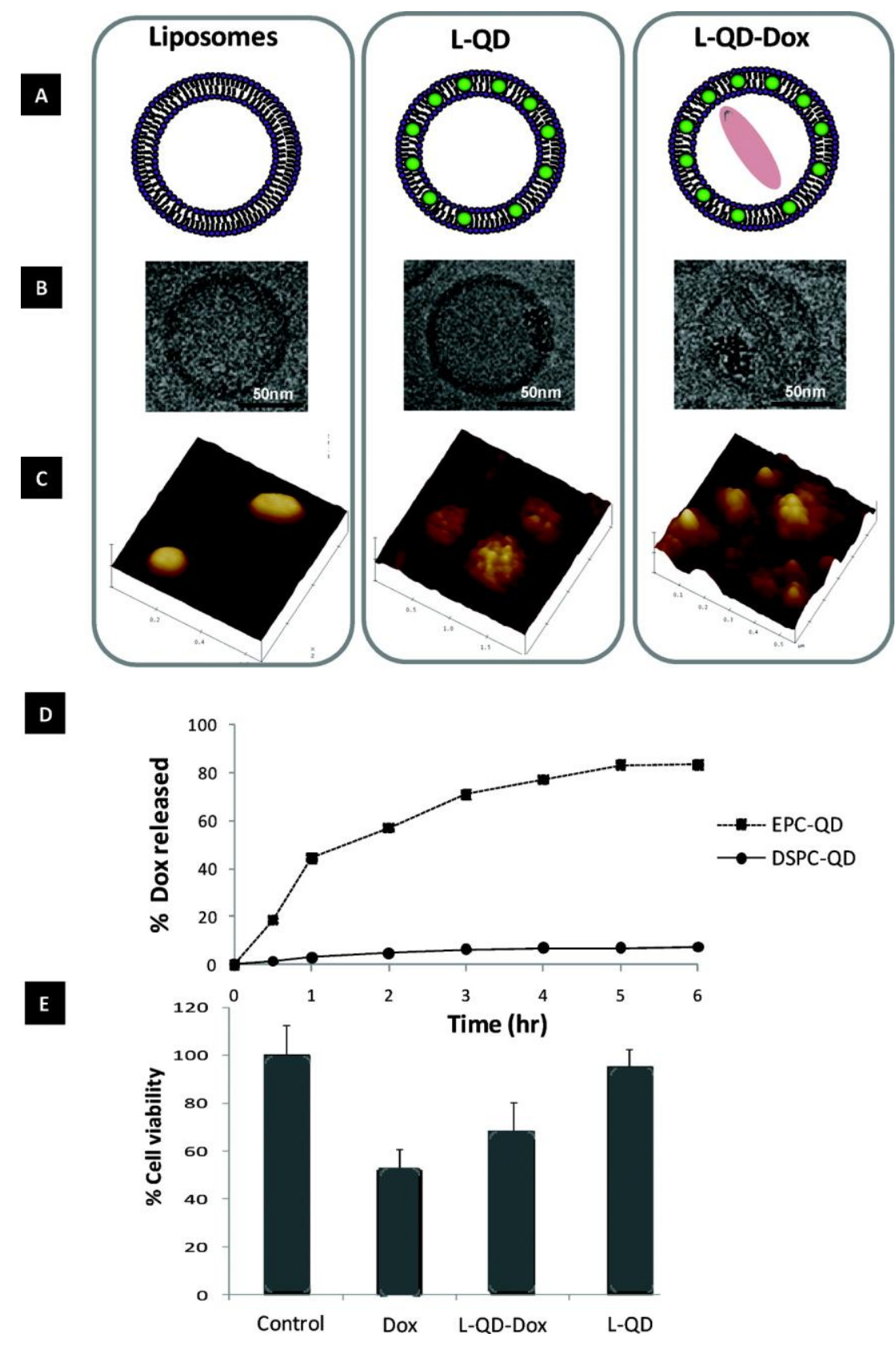

Figure 1-7. Combined imaging and doxorubicin release from D-LNAs prepared with hydrophobic quantum dots (green).

QD embedment and D-LNA structure was verified by cryogenic transmission electron microscopy and atomic force microscopy. The results show that bilayer phase behavior is a key parameter in doxorubicin release; fluid-phase D-LNAs achieved sustained release while gel-phase D-LNAs did not. Reprinted from (Al-Jamal and Kostarelos, 2011) with permission. 


\section{Hyperthermia}

Hyperthermia therapy to treat cancer dates back to Ancient Egyptian medical texts from about 3000 BC (Bolomey et al., 1995). Hyperthermia involves heating the malignant tissues to temperatures between $\sim 40-45^{\circ} \mathrm{C}$. During hyperthermia, heat denatures intracellular proteins inducing death by necrosis or apoptosis. Hyperthermia has also demonstrated the ability to make tumor cells more vulnerable to therapies. Therefore, when hyperthermia is used, it is frequently used in conjunction with chemotherapy and radiotherapy. While the side-effects of hyperthermia are far less severe than chemotherapy and radiotherapy, there is a tendency to damage adjacent tissues. Use of hyperthermia has been tempered in recent years because of difficulty in applying heat to deeper tumors and delivering targeted heating ( $\mathrm{Hsu}$ and $\mathrm{Su}, 2008$; Ito et al., 2004; Pradhan et al., 2010). Development of new therapies may reenergize the field as an effective treatment for malignant tumors. Local hyperthermia has been demonstrated with NPs capable of photothermal and RF heating treatment of malignant tissues (Hildebrandt et al., 2002). Targeting LNAs potentially can provide a local heat source for both hyperthermia and drug release without adversely effecting adjacent tissue (Hsu and $\mathrm{Su}, 2008$; Huang et al., 2011; Ito et al., 2004; Smith et al., 2006).

NPs capable of RF or photothermal heating have been used for local hyperthermia treatment of malignant tissues, which involves heating the tissues to temperatures between $\sim 40-45{ }^{\circ} \mathrm{C}$ (Hildebrandt et al., 2002). During hyperthermia, heat 
denatures intracellular proteins inducing death by necrosis or apoptosis. Hyperthermia has also been shown to make tumor cells more vulnerable to therapies, such as chemotherapy and radiotherapy, therefore it can be used in conjunction with these therapies (Hsu and Su, 2008; Ito et al., 2004; Pradhan et al., 2010). The use of conventional hyperthermia (i.e. without NPs) has been tempered in recent years because of difficulty in applying heat to deeper tumors and delivering targeted heating. This difficulty may be addressed by targeting NPs to malignant cells and tissues.

Heat transfer within tissues via NP heating can be described by a modified Pennes' bio-heat transfer model (Pennes, 1948)

$$
\rho_{t} c_{p, t} \frac{\partial T}{\partial t}=\nabla \cdot\left(k_{t} \nabla T\right)+\rho_{b} c_{p, b} \omega_{b}\left(T-T_{b}\right)+Q_{m}+Q_{N P}
$$

where $\rho_{t}$ is the tissue density and $c_{p, t}$ is the tissue heat capacity. The first term on the right hand side (RHS) of the equation describes the conductive heat transfer $\left(k_{t}\right.$ is the tissue thermal conductivity) and the second term describes the convective heat transfer ( $\rho_{b}$ is the blood density, $c_{p, b}$ is the blood heat capacity, $\omega_{b}$ is the blood perfusion rate, and $T_{b}$ is the blood temperature). $Q_{m}$ is the rate of heat generated metabolically and $Q_{N P}$ is the rate of heat generated from the power dissipation by the NPs, which accounts for the concentration of NPs (e.g. $Q_{N P}$ represented as $S A R$ ). This equation represents the case where the temperature profile in a tissue mass (macro-scale) can be determined as a function of NP concentration and applied field strength (e.g. laser or RF). It has been shown theoretically that sufficient iron oxide NP heating can be 
achieved $\left(>42{ }^{\circ} \mathrm{C}\right)$ at low blood perfusion rates to achieve tissue-level hyperthermia (Rast and Harrison, 2010). For cellular-level heating (nano- or micro-scale), convective heat transfer due to blood transfusion and $Q_{m}$ are eliminated from the equation, yielding the expression reported by Keblinski et al. (Keblinski et al., 2006) for RF NP heating and $\mathrm{Xu}$ et al.(Xu et al., 2007) for $\mathrm{Fe}_{3} \mathrm{O}_{4} \mathrm{NP}$ hyperthermia in vitro.

\section{In vivo and in vitro Biomedical Applications}

\subsection{Cellular Uptake and Drug Delivery}

Chithrani et al. prepared anionic Au decorated liposomes with $105 \mathrm{~nm} d_{\mathrm{h}}$ by incorporating Au-conjugated DPPE (DPPE-Nanogold; $1.4 \mathrm{~nm}$ Au particles) into preformed DPPC/cholesterol liposomes. Incorporation of DPPE-Nanogold was confirmed by TEM and EDS. Liposome uptake by HeLa cells in vitro was independent of the DPPE-Nanogold concentration (2000:1, 1000:1, and 500:1 Au NPs per liposome). This key discovery suggests that the presence of DPPE-Nanogold does not influence cell uptake and that high nanoparticle loadings can be achieved in such LNAs without compromising internalization. Examining the intracellular fate revealed that the Au-liposomes were present in lysosomes and accumulated near the nuclear membrane after incubating for $45 \mathrm{~min}$ (Chithrani et al., 2010).

Paasonen et al. examined uptake and light-induced calcein release of b-LNAs prepared hexanethiol-coated Au NPs (extension of (Paasonen et al., 2007b)). The LNAs were composed of DSPC:DPPC (9:1 molar ratio) and were multilamellar with sizes ranging from 250-370 $\mathrm{nm}$. In vitro studies with human retinal pigment epithelial cell line (ARPE-19) showed that the b-LNAs were internalized by endocytosis and 
localized in endosomes. Exposure to UV light at $400 \mathrm{~mW} / \mathrm{cm}^{2}$ for $300+\mathrm{s}$ led to calcien release from D -LNAs, but not from liposomes that did not contain embedded nanoparticles (Paasonen et al., 2010a).

\subsection{Drug Delivery and Hyperthermia}

Pradhan et al. recently prepared folate (Fol) ligated magnetoliposomes (ELNAs) via thin film hydration that contained co-encapsulated $60 \mathrm{~nm}$ iron oxide NPs and doxorubicin in PBS (Figure 1-8). The lipid components included DPPC, Chol, PEG2000-DSPE, and Fol-PEG2000-DSPE and the liposomes exhibited a melting temperature near $41{ }^{\circ} \mathrm{C}$. The liposomes were $361 \mathrm{~nm}$ in diameter and polydispersed (0.289), with a nanoparticle encapsulation efficiency of $24 \%$ (low, presumably attributed to the use of the thin film hydration method). The liposomes exhibited temperature-dependent doxorubicin release (>2-fold increase from $37^{\circ} \mathrm{C}$ to $43{ }^{\circ} \mathrm{C}$ ). Folate-receptor targeted uptake was demonstrated in HeLa and KB cell cultures, and reductions in cell viability were attributed to a synergistic effect of doxorubicin and hyperthermia treatment achieved in the presence of an EMF. While not mentioned specifically, we speculate that liposomal heating by the nanoparticles may have aided doxorubicin release (Pradhan et al., 2010).

Babincova et al. prepared DPPC/PEG $2000-\mathrm{DSPE}$ LNAs via REV that contained both dextran-coated $\mathrm{Fe}_{3} \mathrm{O}_{4}$ and $\mathrm{C}_{60}$ fullerene (referred to as magnetofullerenosomes) to achieve magnetic targeting and photodynamic therapy (PDT), respectively. Bis(diisobutyloctadecylsiloxy)-2,3-naphthalocyanato silicon (isoBO-SiNc), a photosensitizing agent, was also encapsulated. LNA performance for treating B16 
pigmented melanoma was examined in vivo using a female mouse model. Without magnetic targeting, $\mathrm{C}_{60}-$ mediated PDT followed by isoBO-SiNc-mediated PDT led to a significant reduction in tumor growth over 19 days relative to no treatment or isoBO-SiNc-mediated PDT alone. When magnetic targeting was applied $(0.32 \mathrm{~T}$ magnet placed on the tumor surface for $24 \mathrm{~h}$ ) there was negligible tumor growth over the same duration (Babinec et al., 2005).

\section{Conclusion and Future Outlook}

Liposomes and NPs are both well-established therapeutic and diagnostic agents. As both have been approved for clinical use, the next stage of development is to combine these two systems. LNAs combine the therapeutic advantages of these two nanotechnology systems creating a unique opportunity for achieving multi-functional therapeutic objectives. The liposomes can act to concentrate small NPs and shield them from the immune system. In turn, the NPs can be used to initiate and control drug release when exposed to external stimuli. Recent work demonstrates that there is a range of options for the design of LNAs to provide desired structures and functions. LNAs have been demonstrated to enhance the qualities of encapsulated payloads, by providing a system capable of targeting, responding to external stimuli, and concentrating encapsulates. Also, LNAs are capable of both therapeutic and diagnostic functions.

Nanotechnology is a burgeoning new field, providing solutions to problems that were once considered unsolvable. The multi-functional quality of LNAs makes them a very exciting development in the field of nanotheranostics. However, the 
design and use of LNAs is still in its infancy. Further investigation of the interactions between nanoparticles and the lipid bilayer is necessary to fully understand the formation, structure, and stability of LNAs. For biomedical applications, LNA bioavailability and toxicity need to be studied to grasp the full potential as multimodal nanotechnology therapeutics and diagnostics.

\section{References}

Al-Jamal, W.T., Al-Jamal, K.T., Bomans, P.H., Frederik, P.M., and Kostarelos, K. (2008a). Functionalized-quantum-dot-liposome hybrids as multimodal nanoparticles for cancer. Small 4, 1406-1415.

Al-Jamal, W.T., Al-Jamal, K.T., Tian, B., Lacerda, L., Bomans, P.H., Frederik, P.M., and Kostarelos, K. (2008b). Lipid-quantum dot bilayer vesicles enhance tumor cell uptake and retention in vitro and in vivo. Acs Nano 2, 408-418.

Al-Jamal, W.T., and Kostarelos, K. (2007). Liposome-nanoparticle hybrids for multimodal diagnostic and therapeutic applications. Nanomedicine 2, 85-98.

Al-Jamal, W.T., and Kostarelos, K. (2011). Liposomes: From a Clinically Established Drug Delivery System to a Nanoparticle Platform for Theranostic Nanomedicine. Accounts of Chemical Research 44, 1094-1104.

Anderson, L.J.E., Hansen, E., Lukianova-Hleb, E.Y., Hafner, J.H., and Lapotko, D.O. (2010). Optically guided controlled release from liposomes with tunable plasmonic nanobubbles. Journal of Controlled Release 144, 151-158. 
Babincova, M., Sourivong, P., Leszczynska, D., and Babinec, P. (2003).

Fullerenosomes: Design of a novel nanomaterial for laser controlled topical drug release. Physica Medica 19, 213-216.

Babincova, M., Sourivong, P., Leszczynska, D., and Babinec, P. (2004).

Photodynamic therapy of pigmented melanoma B16 using sterically stabilized fullerenosomes. Laser Phys Lett 1, 476-478.

Babinec, P., Babincova, M., Sourivong, P., and Leszczynska, D. (2005). Efficient treatment of pigmented B16 melanoma using photosensitized long-circulating magnetofullerenosomes. J Magn Magn Mater 293, 394-397.

Bangham, A.D., and Horne, R.W. (1964). Negative staining of phospholipids and their structural modification by surface active agents as observed in the electron microscope. J Mol Biol 8, 660-668.

Bangham, A.D., Standish, M.M., and Watkins, J.C. (1965). Diffusion of univalent ions across the lamellae of swollen phospholipids. J Mol Biol 13, 238-252.

Barenholz, Y. (2012). Doxil® — The first FDA-approved nano-drug: Lessons learned. Journal of Controlled Release.

Bharali, D.J., and Mousa, S.A. (2010). Emerging nanomedicines for early cancer detection and improved treatment: current perspective and future promise. Pharmacol Ther 128, 324-335.

Binder, W.H., Sachsenhofer, R., Farnik, D., and Blaas, D. (2007). Guiding the location of nanoparticles into vesicular structures: a morphological study. Phys Chem Chem Phys 9, 6435-6441. 
Bolomey, J.C., Seegenschmiedt, M.H., Fessenden, P., and Vernon, C.C. (1995). Thermoradiotherapy and thermochemotherapy (Berlin ; New York, Springer). Bothun, G.D. (2008). Hydrophobic silver nanoparticles trapped in lipid bilayers: Size distribution, bilayer phase behavior, and optical properties. Journal of Nanobiotechnology 6, 13.

Bothun, G.D., Lelis, A., Chen, Y., Scully, K., Anderson, L.E., and Stoner, M.A. (2011). Multicomponent folate-targeted magnetoliposomes: design, characterization, and cellular uptake. Nanomedicine: Nanotechnology, Biology and Medicine 7, 797-805.

Bothun, G.D., and Priess, M.R. (2011). Bilayer heating in magnetite nanoparticleliposome dispersions via fluorescence anisotropy. J Coll Inter Sci in press.

Bothun, G.D., Rabideau, A.E., and Stoner, M.A. (2009). Hepatoma cell uptake of cationic multifluorescent quantum dot liposomes. J Phys Chem B 113, 77257728.

Brazel, C.S. (2009). Magnetothermally-responsive nanomaterials: combining magnetic nanostructures and thermally-sensitive polymers for triggered drug release. Pharm Res 26, 644-656.

Brezovich, I.A. (1988). Low frequency hyperthermia. Med Phys Monograph 16, $82-$ 111.

Campbell, R.B., Fukumura, D., Brown, E.B., Mazzola, L.M., Izumi, Y., Jain, R.K., Torchilin, V.P., and Munn, L.L. (2002). Cationic charge determines the 
distribution of liposomes between the vascular and extravascular compartments of tumors. Cancer Res 62, 6831-6836.

Chan, W.C. (1998). Quantum Dot Bioconjugates for Ultrasensitive Nonisotopic Detection. Science 281, 2016-2018.

Chang, H.I., and Yeh, M.K. (2012). Clinical development of liposome-based drugs: formulation, characterization, and therapeutic efficacy. Int J Nanomedicine 7 , $49-60$.

Chen, Y., and Bothun, G.D. (2011). Cationic Gel-Phase Liposomes with "Decorated" Anionic SPIO Nanoparticles: Morphology, Colloidal, and Bilayer Properties. Langmuir 27, 8645-8652.

Chen, Y.J., Bose, A., and Bothun, G.D. (2010). Controlled Release from BilayerDecorated Magnetoliposomes via Electromagnetic Heating. Acs Nano 4, 32153221.

Chen, Y.J., and Bothun, G.D. (2009). Lipid-Assisted Formation and Dispersion of Aqueous and Bilayer-Embedded Nano-C60. Langmuir 25, 4875-4879.

Chithrani, D.B., Dunne, M., Stewart, J., Allen, C., and Jaffray, D.A. (2010). Cellular uptake and transport of gold nanoparticles incorporated in a liposomal carrier'. Nanomed-Nanotechnol 6, 161-169.

Cho, K., Wang, X., Nie, S., Chen, Z.G., and Shin, D.M. (2008). Therapeutic nanoparticles for drug delivery in cancer. Clin Cancer Res 14, 1310-1316.

Corchero, J.L., and Villaverde, A. (2009). Biomedical applications of distally controlled magnetic nanoparticles. Trends Biotechnol 27, 468-476. 
Crossley, K.B. (2010). Staphylococci in human disease, 2nd edn (Chichester, West Sussex ; Hoboken, NJ, Wiley-Blackwell).

Dandamudi, S., Patil, V., Fowle, W., Khaw, B.-A., and Campbell, R.B. (2009). External magnet improves antitumor effect of vinblastine and the suppression of metastasis. Cancer Science 100, 1537-1543.

Danhier, F., Feron, O., and Préat, V. (2010). To exploit the tumor microenvironment: Passive and active tumor targeting of nanocarriers for anti-cancer drug delivery. Journal of Controlled Release 148, 135-146.

De Cuyper, M., and Joniau, M. (1988). Magnetoliposomes: Formation and structural characterization. Eur Biophys J 15, 311-319.

Doi, Y., Ikeda, A., Akiyama, M., Nagano, M., Shigematsu, T., Ogawa, T., Takeya, T., and Nagasaki, T. (2008). Intracellular uptake and photodynamic activity of water-soluble [60]- and [70]fullerenes incorporated in liposomes. Chem--Eur J 14, 8892-8897.

Dudu, V., Ramcharan, M., Gilchrist, M.L., Holland, E.C., and Vazquez, M. (2008). Liposome delivery of quantum dots to the cytosol of live cells. J Nanosci Nanotechnol 8, 2293-2300.

Emerich, D.F., and Thanos, C.G. (2006). The pinpoint promise of nanoparticle-based drug delivery and molecular diagnosis. Biomol Eng 23, 171-184.

Fattahi, H., Laurent, S., Liu, F., Arsalani, N., Elst, L.V., and Muller, R.N. (2011). Magnetoliposomes as multimodal contrast agents for molecular imaging and cancer nanotheragnostics. Nanomedicine 6, 529-544. 
Fortin-Ripoche, J.P., Martina, M.S., Gazeau, F., Menager, C., Wilhelm, C., Bacri, J.C., Lesieur, S., and Clement, O. (2006). Magnetic Targeting of Magnetoliposomes to Solid Tumors with MR Imaging Monitoring in Mice: Feasibility. Radiology 239, 415-424.

Fukumori, Y., and Ichikawa, H. (2006). Nanoparticles for cancer therapy and diagnosis. Advanced Powder Technology 17, 1-28.

Gannon, C.J., Patra, C.R., Bhattacharya, R., Mukherjee, P., and Curley, S.A. (2008). Intracellular gold nanoparticles enhance non-invasive radiofrequency thermal destruction of human gastrointestinal cancer cells. J Nanobiotechnology 6, 2.

Gibaldi, M., Lee, M., Desai, A., and American Society of Health-System Pharmacists. (2007). Gibaldi's drug delivery systems in pharmaceutical care (Bethesda, Md., American Society of Health-System Pharmacists).

Ginzburg, V.V., and Balijepalli, S. (2007). Modeling the thermodynamics of the interaction of nanoparticles with cell membranes. Nano Lett 7, 3716-3722.

Gomes, J.F.P.S., Rank, A., Kronenberger, A., Fritz, J., Winterhalter, M., and Ramaye, Y. (2009). Polyelectrolyte-coated unilamellar nanometer-sized magnetic liposomes. Langmuir 25, 6793-6799.

Gopalakrishnan, G., Danelon, C., Izewska, P., Prummer, M., Bolinger, P.-Y., Geissbühler, I., Demurtas, D., Dubochet, J., and Vogel, H. (2006). Multifunctional Lipid/Quantum Dot Hybrid Nanocontainers for Controlled Targeting of Live Cells. Angewandte Chemie International Edition 45, 54785483. 
Goyal, P., Goyal, K., Vijaya Kumar, S.G., Singh, A., Katare, O.P., and Mishra, D.N. (2005). Liposomal drug delivery systems--clinical applications. Acta Pharm $55,1-25$.

Gregoriadis, G. (1973). Drug entrapment in liposomes. FEBS Lett 36, 292-296.

Groneberg, D.A., Giersig, M., Welte, T., and Pison, U. (2006). Nanoparticle-based diagnosis and therapy. Curr Drug Targets 7, 643-648.

Gullotti, E., and Yeo, Y. (2009). Extracellularly Activated Nanocarriers: A New Paradigm of Tumor Targeted Drug Delivery. Molecular Pharmaceutics 6, 1041-1051.

Hildebrandt, B., Wust, P., Ahlers, O., Dieing, A., Sreenivasa, G., Kerner, T., Felix, R., and Riess, H. (2002). The cellular and molecular basis of hyperthermia. Crit Rev Oncol Hematol 43, 33-56.

Hong, K., Friend, D.S., Glabe, C.G., and Papahadjopoulos, D. (1983). Liposomes containing colloidal gold are a useful probe of liposome-cell interactions. Biochim Biophys Acta 732, 320-323.

Hsu, M.H., and Su, Y.C. (2008). Iron-oxide embedded solid lipid nanoparticles for magnetically controlled heating and drug delivery. Biomed Microdevices 10, 785-793.

Huang, H.-C., Barua, S., Sharma, G., Dey, S.K., and Rege, K. (2011). Inorganic nanoparticles for cancer imaging and therapy. Journal of Controlled Release $155,344-357$. 
Huang, X., El-Sayed, I.H., and El-Sayed, M.A. (2010). Applications of gold nanorods for cancer imaging and photothermal therapy. Methods Mol Biol 624, 343-357.

Hwang, K.C., and Mauzerall, D. (1993). Photoinduced Electron-Transport across a Lipid Bilayer Mediated by C70. Nature 361, 138-140.

Ikeda, A., and Kikuchi, J.-I. (2008). Fullerene C70-containing liposome, method for producing the same, and use of the same (National University Corporation Nara Institute of Science and Technology, Japan .), pp. 47pp.

Ikeda, A., Nagano, M., Akiyama, M., Matsumoto, M., Ito, S., Mukai, M., Hashizume, M., Kikuchi, J.-i., Katagiri, K., Ogawa, T., et al. (2009). Photodynamic activity of C70 caged within surface-cross-linked liposomes. Chem--Asian J 4, 199205.

Ikeda, A., Sato, T., Kitamura, K., Nishiguchi, K., Sasaki, Y., Kikuchi, J., Ogawa, T., Yogo, K., and Takeya, T. (2005). Efficient photocleavage of DNA utilising water-soluble lipid membrane-incorporated [60]fullerenes prepared using a [60]fullerene exchange method. Org Biomol Chem 3, 2907-2909.

Immordino, M.L., Dosio, F., and Cattel, L. (2006). Stealth liposomes: review of the basic science, rationale, and clinical applications, existing and potential. Int $\mathrm{J}$ Nanomed 1, 297-315.

Ishida, T., Harashima, H., and Kiwada, H. (2002). Liposome clearance. Biosci Rep 22, 197-224.

Ito, A., Kuga, Y., Honda, H., Kikkawa, H., Horiuchi, A., Watanabe, Y., and Kobayashi, T. (2004). Magnetite nanoparticle-loaded anti-HER2 
immunoliposomes for combination of antibody therapy with hyperthermia. Cancer Letters 212, 167-175.

Ito, A., Shinkai, M., Honda, H., and Kobayashi, T. (2005). Medical application of functionalized magnetic nanoparticles. J Biosci Bioeng 100, 1-11.

Jang, H., Pell, L.E., Korgel, B.A., and English, D.S. (2003). Photoluminescence quenching of silicon nanoparticles in phospholipid vesicle bilayers. $\mathbf{J}$ Photochem Photobiol A 158, 111-117.

Jeng, U.-S., Hsu, C.-H., Lin, T.-L., Wu, C.-M., Chen, H.-L., Tai, L.-A., and Hwang, K.-C. (2005). Dispersion of fullerenes in phospholipid bilayers and the subsequent changes in the host bilayers. Physica B 357, 193-198.

Jin, S., and Ye, K. (2007). Nanoparticle-mediated drug delivery and gene therapy. Biotechnol Prog 23, 32-41.

Jørgensen, L., and Nielsen, H.M. (2009). Delivery technologies for biopharmaceuticals : peptides, proteins, nucleic acids and vaccines (Chichester, U.K., Wiley).

Kamaly, N., Kalber, T., Thanou, M., Bell, J.D., and Miller, A.D. (2009). Folate receptor targeted bimodal liposomes for tumor magnetic resonance imaging. Bioconjug Chem 20, 648-655.

Keblinski, P., Cahill, D.G., Bodapati, A., Sullivan, C.R., and Taton, T.A. (2006). Limits of localized heating by electromagnetically excited nanoparticles. J Appl Phys 100,-- 
Kikumori, T., Kobayashi, T., Sawaki, M., and Imai, T. (2008). Anti-cancer effect of hyperthermia on breast cancer by magnetite nanoparticle-loaded anti-HER2 immunoliposomes. Breast Cancer Research and Treatment 113, 435-441.

Kirat, K.E., Morandat,S., Dufrene, Y.F. (2010). Nanoscale analysis of supported lipid bilayers using atomic force microscopy. Biochimica et Biophysica Acta 1798, 750-765.

Kojima, C., Hirano, Y., Yuba, E., Harada, A., and Kono, K. (2008a). Preparation and characterization of complexes of liposomes with gold nanoparticles. Coll Surf B $66,246-252$.

Kojima, C., Hirano, Y., Yuba, E., Harada, A., and Kono, K. (2008b). Preparation and characterization of complexes of liposomes with gold nanoparticles. Colloids and Surfaces B-Biointerfaces 66, 246-252.

Kola, I., and Landis, J. (2004). Opinion: Can the pharmaceutical industry reduce attrition rates? Nature Reviews Drug Discovery 3, 711-716.

Koo, O.M., Rubinstein, I., and Onyuksel, H. (2005). Role of nanotechnology in targeted drug delivery and imaging: a concise review. Nanomedicine: Nanotechnology, Biology and Medicine 1, 193-212.

Krpetic, Z., Nativo, P., See, V., Prior, I.A., Brust, M., and Volk, M. (2010). Inflicting Controlled Nonthermal Damage to Subcellular Structures by Laser-Activated Gold Nanoparticles. Nano Letters 10, 4549-4554. 
Kshirsagar, N.A., Pandya, S.K., Kirodian, G.B., and Sanath, S. (2005). Liposomal drug delivery system from laboratory to clinic. J Postgrad Med 51 Suppl 1, S515.

Kullberg, M., Mann, K., and Owens, J.L. (2005). Improved drug delivery to cancer cells: a method using magnetoliposomes that target epidermal growth factor receptors. Med Hypotheses 64, 468-470.

Kullberg, M., Mann, K., and Owens, J.L. (2009). A two-component drug delivery system using Her-2-targeting thermosensitive liposomes. J Drug Target 17, 98107.

L.F. Pavon, L.F.G., L.C. Marti, E. Amaro, C.A. Moreira, M.I. Camargo-Mathias, O.K. Okamoto (2008). Ultrastructural characterization of CD133(+) stem cells29 bound to superparamagnetic nanoparticles: possible biotechnological applications. Journal of Microscopy-Oxford 231, 374-383.

Larsen, B.A., Haag, M.A., Serkova, N.J., Shroyer, K.R., and Stoldt, C.R. (2008). Controlled aggregation of superparamagnetic iron oxide nanoparticles for the development of molecular magnetic resonance imaging probes. Nanotechnology 19, -.

Laurent, S., Forge, D., Port, M., Roch, A., Robic, C., Vander Elst, L., and Muller, R.N. (2008). Magnetic iron oxide nanoparticles: synthesis, stabilization, vectorization, physicochemical characterizations, and biological applications. Chem Rev 108, 2064-2110. 
Leckband, D., Israelachvili,J. (2001). Intermolecular forces in biology. Quarterly Reviews of Biophysics 34, 105-267.

Leeson, P.D., and Davis, A.M. (2004). Time-Related Differences in the Physical Property Profiles of Oral Drugs. Journal of Medicinal Chemistry 47, 63386348.

Li, J.L., and Gu, M. (2010). Gold-Nanoparticle-Enhanced Cancer Photothermal Therapy. Ieee J Sel Top Quant 16, 989-996.

Lipowsky, R., and Dobereiner, H.G. (1998). Vesicles in contact with nanoparticles and colloids. Europhys Lett 43, 219-225.

Lu, Y., and Low, P.S. (2002). Folate-mediated delivery of macromolecular anticancer therapeutic agents. Advanced Drug Delivery Reviews 54, 675-693.

Mady, M.M., Fathy, M.M., Youssef, T., and Khalil, W.M. (2011). Biophysical characterization of gold nanoparticles-loaded liposomes. Physica Medica.

Malam, Y., Loizidou, M., and Seifalian, A.M. (2009). Liposomes and nanoparticles: nanosized vehicles for drug delivery in cancer. Trends Pharmacol Sci 30, 592599.

Marchenko, I., Yashchenok,A., German,S., Inozemtseva,O., Gorin,D., Bukreeva,T., Mohwald, H., Skirtach,A. (2010). Polyelecrolytes: Influence of evaporative self-assembly of particle and assembly of multilayres with polymers, nanoparticle, and carnon nanotubes. Polymers 2, 690-708.

Mart, R.J., Liem, K.P., and Webb, S.J. (2009). Creating Functional Vesicle Assemblies from Vesicles and Nanoparticles. Pharm Res-Dord 26, 1701-1710. 
Maurer, N., Fenske, D.B., and Cullis, P.R. (2001). Developments in liposomal drug delivery systems. Expert Opin Biol Ther 1, 923-947.

McCarthy, J., and Weissleder, R. (2008). Multifunctional magnetic nanoparticles for targeted imaging and therapy $\underset{乛}{ }$. Advanced Drug Delivery Reviews 60, 12411251.

Medina, O.P., Zhu, Y., and Kairemo, K. (2004). Targeted liposomal drug delivery in cancer. Curr Pharm Des 10, 2981-2989.

Michalet, X., Pinaud, F.F., Bentolila, L.A., Tsay, J.M., Doose, S., Li, J.J., Sundaresan, G., Wu, A.M., Gambhir, S.S., and Weiss, S. (2005). Quantum dots for live cells, in vivo imaging, and diagnostics. Science 307, 538-544.

Minko, T., Pakunlu, R.I., Wang, Y., Khandare, J.J., and Saad, M. (2006). New generation of liposomal drugs for cancer. Anticancer Agents Med Chem 6, $537-552$.

Moen, M.D., Lyseng-Williamson, K.A., and Scott, L.J. (2009). Liposomal amphotericin B: a review of its use as empirical therapy in febrile neutropenia and in the treatment of invasive fungal infections. Drugs 69, 361-392.

Moghimi, S.M., and Szebeni, J. (2003). Stealth liposomes and long circulating nanoparticles: critical issues in pharmacokinetics, opsonization and proteinbinding properties. Prog Lipid Res 42, 463-478.

Mornet, S., Vasseur, S., Grasset, F., and Duguet, E. (2004). Magnetic nanoparticle design for medical diagnosis and therapy. J Mater Chem 14, 2161-2175. 
Mulder, W.J., Strijkers, G.J., van Tilborg, G.A., Griffioen, A.W., and Nicolay, K. (2006). Lipid-based nanoparticles for contrast-enhanced MRI and molecular imaging. NMR Biomed 19, 142-164.

Murakami, Y., Nakano, A., Yoshimatsu, A., Uchitomi, K., and Matsuda, Y. (1984). Characterization of Molecular Aggregates of Peptide Amphiphiles and Kinetics of Dynamic Processes Performed by Single-Walled Vesicles. Journal Of The American Chemical Society 106, 3613-3623.

N. Li, D.S.K., Y. Lvov, W. Liebenberg, L.R. Tiedt, M.M. De Villiers, (2006). Nanoparticle multilayers: Surface modification of photosensitive drug microparticles for increased stability and in vitro bioavailability. Journal of Nanoscience and Nanotechnology 6, 3252-3260.

National Cancer Institute. National Cancer Institute, 2005.

Neuberger, T., Schöpf, B., Hofmann, H., Hofmann, M., and von Rechenberg, B. (2005). Superparamagnetic nanoparticles for biomedical applications: Possibilities and limitations of a new drug delivery system. Journal of Magnetism and Magnetic Materials 293, 483-496.

Niu, S.F., and Manzerall, D. (1996). Fast and efficient charge transport across a lipid bilayer is electronically mediated by C-70 fullerene aggregates. Journal Of The American Chemical Society 118, 5791-5795.

Oldenburg, S.J., Jackson, J.B., Westcott, S.L., and Halas, N.J. (1999). Infrared extinction properties of gold nanoshells. Appl Phys Lett 75, 2897-2899. 
Paasonen, L., Laaksonen, T., Johans, C., Yliperttula, M., Kontturi, K., and Urth, A. (2007a). Gold nanoparticles enable selective light-induced contents release from liposomes. Journal of Controlled Release 122, 86-93.

Paasonen, L., Laaksonen, T., Johans, C., Yliperttula, M., Kontturi, K., and Urth, A. (2007b). Gold nanoparticles enable selective light-induced contents release from liposomes. J Control Release 122, 86-93.

Paasonen, L., Sipila, T., Subrizi, A., Laurinmaki, P., Butcher, S.J., Rappolt, M., Yaghmur, A., Urtti, A., and Yliperttula, M. (2010a). Gold-embedded photosensitive liposomes for drug delivery: Triggering mechanism and intracellular release. J Control Release 147, 136-143.

Paasonen, L., Sipila, T., Subrizi, A., Laurinmaki, P., Butcher, S.J., Rappolt, M., Yaghmur, A., Urtti, A., and Yliperttula, M. (2010b). Gold-embedded photosensitive liposomes for drug delivery: Triggering mechanism and intracellular release. Journal of Controlled Release 147, 136-143.

Pagano, R.E., and Weinstein, J.N. (1978). Interactions of Liposomes with Mammalian-Cells. Annu Rev Biophys Bio 7, 435-468.

Pankhurst, Q.A., Connolly, J., Jones, S.K., and Dobson, J. (2003). Applications of magnetic nanoparticles in biomedicine. J Phys D 36, 167-181.

Papahadjopoulos, D., and Ohki, S. (1969). Stability of asymmetric phospholipid membranes. Science 164, 1075-1077. 
Park, S.-H., Oh, S.-G., Mun, J.-Y., and Han, S.-S. (2005). Effects of silver nanoparticles on the fluidity of bilayer in phospholipid liposome. Coll Surf B $44,117-122$.

Park, S.-H., Oh, S.-G., Mun, J.-Y., and Han, S.-S. (2006). Loading of gold nanoparticles inside the DPPC bilayers of liposome and their effects on membrane fluidities. Coll Surf B 48, 112-118.

Pelicano, H., Martin, D.S., Xu, R.H., and Huang, P. (2006). Glycolysis inhibition for anticancer treatment. Oncogene 25, 4633-4646.

Pennes, H.H. (1948). Analysis of tissue and arterial temperature in the resting human forearm. J Appl Physiol 1, 93-122.

Polyak, B., and Friedman, G. (2009). Magnetic targeting for site-specific drug delivery: applications and clinical potential. Expert Opin Drug Deliv 6, 53-70.

Ponce, A.M., Viglianti, B.L., Yu, D., Yarmolenko, P.S., Michelich, C.R., Woo, J., Bally, M.B., and Dewhirst, M.W. (2007). Magnetic Resonance Imaging of Temperature-Sensitive Liposome Release: Drug Dose Painting and Antitumor Effects. JNCI Journal of the National Cancer Institute 99, 53-63.

Pornpattananangkul, D., Olson, S., Aryal, S., Sartor, M., Huang, C.M., Vecchio, K., and Zhang, L. (2010). Stimuli-Responsive Liposome Fusion Mediated by Gold Nanoparticles. Acs Nano 4, 1935-1942.

Pornpattananangkul, D., Zhang, L., Olson, S., Aryal, S., Obonyo, M., Vecchio, K., Huang, C.-M., and Zhang, L. (2011). Bacterial Toxin-Triggered Drug Release 
from Gold Nanoparticle-Stabilized Liposomes for the Treatment of Bacterial Infection. Journal of the American Chemical Society 133, 4132-4139.

Pradhan, P., Giri, J., Banerjee, R., Bellage, J., and Bahadur, D. (2007). Preparation and characterization of manganese ferrite-based magnetic liposomes for hyperthermia treatment of cancer. J Magn Magn Mater 311, 208-215.

Pradhan, P., Giri, J., Rieken, F., Koch, C., Mykhaylyk, O., Döblinger, M., Banerjee, R., Bahadur, D., and Plank, C. (2010). Targeted temperature sensitive magnetic liposomes for thermo-chemotherapy. Journal of Controlled Release $142,108-121$.

Preiss, M.R., and Bothun, G.D. (2011). Stimuli-responsive liposome-nanoparticle assemblies. Expert Opinion on Drug Delivery 8, 1025-1040.

Puri, A., Loomis, K., Smith, B., Lee, J.H., Yavlovich, A., Heldman, E., and Blumenthal, R. (2009). Lipid-based nanoparticles as pharmaceutical drug carriers: from concepts to clinic. Crit Rev Ther Drug Carrier Syst 26, 523-580.

Rasch, M.R., Rossinyol, E., Hueso, J.L., Goodfellow, B.W., Arbiol, J., and Korgel, B.A. (2010a). Hydrophobic Gold Nanoparticle Self-Assembly with Phosphatidylcholine Lipid: Membrane-Loaded and Janus Vesicles. Nano Letters 10, 3733-3739.

Rasch, M.R., Rossinyol, E., Hueso, J.L., Goodfellow, B.W., Arbiol, J., and Korgel, B.A. (2010b). Hydrophobic Gold Nanoparticle Self-Assembly with Phosphatidylcholine Lipid: Membrane-Loaded and Janus Vesicles. Nano Lett $10,3733-3739$. 
Rast, L., and Harrison, J.G. (2010). Computational modeling of electromagnetically induced heating of magnetic manoparticle materials for hyperthermic cancer treatment. PIERS Online 6, 690-694.

Rivera Gil, P., Hühn, D., del Mercato, L.L., Sasse, D., and Parak, W.J. (2010). Nanopharmacy: Inorganic nanoscale devices as vectors and active compounds. Pharmacological Research 62, 115-125.

Riviere, C., Martina, M.S., Tomita, Y., Wilhelm, C., Tran Dinh, A., Menager, C., Pinard, E., Lesieur, S., Gazeau, F., and Seylaz, J. (2007). Magnetic targeting of nanometric magnetic fluid loaded liposomes to specific brain intravascular areas: a dynamic imaging study in mice. Radiology 244, 439-448.

Roiter, Y., Ornatska, M., Rammohan, A.R., Balakrishnan, J., Heine, D.R., and Minko, S. (2008). Interaction of Nanoparticles with Lipid Membrane. Nano Letters 8 , 941-944.

Rotomskis, R., Streckyte, G., and Karabanovas, V. (2006). [Nanoparticles in diagnostics and therapy: towards nanomedicine]. Medicina (Kaunas) 42, 542558.

S. Jin, K.Y. (2007). Nanoparticle-mediated drug delivery and gene therapy. Biotechnology Progress 23, 32-41.

S. Legrand, A.C., L. Kind, E.C. Constable, C.E. Housecroft, L. Landmann, P. Banse, U. Pielesa, A. Wirth-Heller (2008). Controlling silica nanoparticle properties for biomedical applications through surface modification. New Journal of Chemistry 32, 588-593. 
Sabate, R., Barnadas-Rodriguez, R., Callejas-Fernandez, J., Hidalgo-Alvarez, R., and Estelrich, J. (2008). Preparation and characterization of extruded magnetoliposomes. Int J Pharm 347, 156-162.

Samad, A., Sultana, Y., and Aqil, M. (2007). Liposomal drug delivery systems: an update review. Curr Drug Deliv 4, 297-305.

Sassaroli, E., Li, K.C.P., and O'Neill, B.E. (2009). Numerical investigation of heating of a gold nanoparticle and the surrounding microenvironment by nanosecond laser pulses for nanomedicine applications. Physics in Medicine and Biology $54,5541-5560$.

Sau, T.P., Urban, A.S., Dondapati, S.K., Fedoruk, M., Horton, M.R., Rogach, A.L., Stefani, F.D., Radler, J.O., and Feldmann, J. (2009). Controlling loading and optical properties of gold nanoparticles on liposome membranes. Coll Surf A 342, 92-96.

Shinkai, M., Yanase, M., Honda, H., Wakabayashi, T., Yoshida, J., and Kobayashi, T. (1996). Intracellular hyperthermia for cancer using magnetite cationic liposomes: In vitro study. Jpn J Cancer Res 87, 1179-1183.

Sivashankar, M., Katyayani, T. (2011). Liposomes-the future of formulations. International Journal of research pharmacy and chemistry 1, 259-267.

Smith, A.M., Dave, S., Nie, S., True, L., and Gao, X. (2006). Multicolor quantum dots for molecular diagnostics of cancer. Expert Review of Molecular Diagnostics $6,231-244$. 
Sun, C., Lee, J.S., and Zhang, M. (2008). Magnetic nanoparticles in MR imaging and drug delivery. Adv Drug Deliv Rev 60, 1252-1265.

Szoka, F., Jr., and Papahadjopoulos, D. (1978). Procedure for preparation of liposomes with large internal aqueous space and high capture by reverse-phase evaporation. Proc Natl Acad Sci USA 75, 4194-4198.

Tai, L.A., Tsai, P.J., Wang, Y.C., Wang, Y.J., Lo, L.W., and Yang, C.S. (2009). Thermosensitive liposomes entrapping iron oxide nanoparticles for controllable drug release. Nanotechnology 20, -.

Teja, A.S., and Koh, P.-Y. (2009). Synthesis, properties, and applications of magnetic iron oxide nanoparticles. Progress in Crystal Growth and Characterization of Materials 55, 22-45.

Thorek, D.L.J., Chen, A.K., Czupryna, J., and Tsourkas, A. (2006).

Superparamagnetic Iron Oxide Nanoparticle Probes for Molecular Imaging. Annals of Biomedical Engineering 34, 23-38.

Tian, B., Al-Jamal, W.T., Al-Jamal, K.T., and Kostarelos, K. (2011). Doxorubicinloaded lipid-quantum dot hybrids: Surface topography and release properties. Int J Pharm 416, 443-447.

Torchilin, V.P. (2005). Recent advances with liposomes as pharmaceutical carriers. Nat Rev Drug Discov 4, 145-160.

van Vlerken, L.E., and Amiji, M.M. (2006). Multi-functional polymeric nanoparticles for tumour-targeted drug delivery. Expert Opin Drug Deliv 3, 205-216. 
Veiseh, O., Gunn, J.W., and Zhang, M. (2010). Design and fabrication of magnetic nanoparticles for targeted drug delivery and imaging. Advanced Drug Delivery Reviews 62, 284-304.

Volodkin, D.V., Skirtach, A.G., and Mohwald, H. (2009). Near-IR Remote Release from Assemblies of Liposomes and Nanoparticles. Angew Chem Int Ed 48, 1807-1809.

Walling, M.A., Novak, J.A., and Shepard, J.R.E. (2009). Quantum Dots for Live Cell and In Vivo Imaging. International Journal of Molecular Sciences 10, 441-491.

Wang, B., Zhang, L.F., Bae, S.C., and Granick, S. (2008). Nanoparticle-induced surface reconstruction of phospholipid membranes. Proc Nat Acad Sci USA $105,18171-18175$.

Weng, K.C., Noble, C.O., Papahadjopoulos-Sternberg, B., Chen, F.F., Drummond, D.C., Kirpotin, D.B., Wang, D., Hom, Y.K., Hann, B., and Park, J.W. (2008). Targeted tumor cell internalization and imaging of multifunctional quantum dot-conjugated immunoliposomes in vitro and in vivo. Nano Lett 8, 2851 2857.

Wi, H.S., Lee, K., and Pak, H.K. (2008). Interfacial energy consideration in the organization of a quantum dot-lipid mixed system. J Phys Cond Mat 20, 1-6.

Wijaya, A., and Hamad-Schifferli, K. (2007). High-Density Encapsulation of Fe3O4Nanoparticles in Lipid Vesicles. Langmuir 23, 9546-9550. 
Wu, G.H., Milkhailovsky, A., Khant, H.A., Fu, C., Chiu, W., and Zasadzinski, J.A. (2008). Remotely triggered liposome release by near-infrared light absorption via hollow gold nanoshells. J Am Chem Soc 130, 8175-8177.

Xu, R.Z., Zhang, Y., Ma, M., Xia, J.G., Liu, J.W., Guo, Q.Z., and Gu, N. (2007). Measurement of specific absorption rate and thermal simulation for arterial embolization hyperthermia in the Maghemite-Gelled model. Ieee T Magn 43, 1078-1085.

Yan, X., Scherphof, G.L., and Kamps, J.A.A.M. (2005). Liposome Opsonization. Journal of Liposome Research 15, 109-139.

Yang, C., Rait, A., Pirollo, K.F., Dagata, J.A., Farkas, N., and Chang, E.H. (2008). Nanoimmunoliposome delivery of superparamagnetic iron oxide markedly enhances targeting and uptake in human cancer cells in vitro and in vivo. Nanomedicine: Nanotechnology, Biology and Medicine 4, 318-329.

Yu, Y., Anthony, S.M., Zhang, L., Bae, S.C., and Granick, S. (2007). Cationic nanoparticles stabilize zwitterionic liposomes better than anionic ones. J Phys Chem C 111, 8233-8236.

Yu, Y., and Granick, S. (2009). Pearling of Lipid Vesicles Induced by Nanoparticles. Journal of the American Chemical Society 131, 14158-14159.

Zhang, L., Gu, F.X., Chan, J.M., Wang, A.Z., Langer, R.S., and Farokhzad, O.C. (2008). Nanoparticles in medicine: therapeutic applications and developments. Clin Pharmacol Ther 83, 761-769. 
Zhang, L.F., and Granick, S. (2006). How to stabilize phospholipid liposomes (using nanoparticles). Nano Lett 6, 694-698.

Zhang, S.L., Li, J., Lykotrafitis, G., Bao, G., and Suresh, S. (2009). Size-Dependent Endocytosis of Nanoparticles. Adv Mater 21, 419-+.

Zheng, S., Zheng, Y., Beissinger, R.L., and Fresco, R. (1994). Microencapsulation of Hemoglobin in Liposomes Using a Double Emulsion, Film Dehydration Rehydration Approach. Bba-Biomembranes 1196, 123-130.

Zheng, X., Lu, J., Deng, L., Xiong, Y., and Chen, J. (2009). Preparation and characterization of magnetic cationic liposome in gene delivery. Int J Pharm $366,211-217$.

Zhu, L., Huo, Z.L., Wang, L.L., Tong, X., Xiao, Y., and Ni, K.Y. (2009). Targeted delivery of methotrexate to skeletal muscular tissue by thermosensitive magnetoliposomes. International Journal of Pharmaceutics 370, 136-143. 


\title{
CHAPTER 2
}

\section{Stimuli-Responsive Liposome-Nanoparticle Assemblies}

\author{
Matthew R Preiss ${ }^{1}$ and Geoffrey D Bothun ${ }^{1 \dagger}$ \\ Published in: Expert Opinion on Drug Delivery 8(8) 1025-1040 (2011).
${ }^{1}$ University of Rhode Island, Department of Chemical Engineering, Rhode Island Consortium for Nanoscience and Nanotechnology, \\ Kingston, RI, USA
}

${ }^{\dagger}$ Author for correspondence: Geoffrey D Bothun

Department of Chemical Engineering

University of Rhode Island

205 Crawford Hall, 16 Greenhouse Road,

Kingston, RI, 02881, USA

Phone: +1-401-874-9518

Email: gbothun@uri.edu 


\section{Abstract}

\subsection{Introduction:}

Nanoscale assemblies are needed that achieve multiple therapeutic objectives including cellular targeting, imaging, diagnostics, and drug delivery. These must exhibit high stability, bioavailability, and biocompatibility, while maintaining or enhancing the inherent activity of the therapeutic cargo. Liposome-nanoparticle assemblies (LNAs) combine the demonstrated potential of liposome-based therapies with functional nanoparticles. Specifically, LNAs can be used to concentrate and shield the nanoparticles and, in turn, stimuli-responsive nanoparticles that respond to external fields can be used to control liposomal release. The ability to design LNAs via nanoparticle encapsulation, decoration, or bilayer-embedment offers a range of structures with different structures and functions.

\subsection{Areas Covered:}

The current state of research and understanding of the design, characterization, and performance of LNAs. Brief reviews are provided for liposomes and nanoparticles for therapeutic application, followed by a discussion of the opportunities and challenges associated with combining the two in a single assembly to achieve controlled release via light or radiofrequency stimuli.

\subsection{Expert Opinion:}

LNAs offer a unique opportunity to combine the therapeutic properties of liposomes and nanoparticles. Liposomes act to concentrate small nanoparticles and 
shield nanoparticles from the immune system while, the nanoparticle can be used to initiate and control drug release when exposed to external stimuli. These properties provide a platform to achieve nanoparticle-controlled liposomal release. LNA design and application is still in its infancy. Research concentrating on the relationships between LNA structure, function, and performance is essential for future clinical use of LNAs.

Keywords: liposome, nanoparticle, photothermal, electromagnetic, controlled release 


\section{Article Highlights.}

- Review of recently reported liposome-nanoparticle assemblies (LNAs) designed for stimuli-responsive controlled-release. Radio frequency-triggered magnetoliposomes are a classic example of a stimuli-responsive LNA. In recent years, there has been an increased interest in LNA designs utilizing lightresponsive nanoparticles, such as gold nanoparticles.

- Three distinct strategies are used to design LNAs: nanoparticle encapsulation, bilayer-embedment, or surface decoration. With each design, nanoparticleliposome interactions must be considered as they play a role in LNA structure and stability.

- Intuitively, LNA release in the presence of an electromagnetic field is enhanced when the nanoparticle is closely associated with the bilayer. Examples are presented from the literature for iron oxide and gold nanoparticles.

- LNA release is commonly attributed to local nanoparticle heating; however, mechanically-induced release may be more plausible.

This box summarizes key points contained in the article.

\section{Introduction}

A significant challenge faced today in drug discovery is that many promising therapeutics have poor pharmacological properties, making them unsuitable for use in their native forms [1]. Some estimate that greater than $95 \%$ of new drug candidates 
fail to have the pharmacokinetic properties needed to be an effective treatment [2]. Improving pharmacokinetics requires chemically modifying the drug, for instance to make it water soluble, or physically modifying it by mixing or encapsulating it within a suitable matrix. Disconnect between drug discovery and drug delivery is one of the biggest reasons for the decline in breakthrough drugs in recent years [1]. New nanotechnology-based drug delivery systems have shown great potential for overcoming obstacles related to poor pharmacokinetics by providing a mechanism for controlling the delivering of low drug dosages to specific tissues or cells [3, 4]. Targeted and controlled delivery can reduce the adverse effects of systemic delivery and off-target affects. The dream of Nobel Laureate Paul Ehrlich's "magic bullet" may be within reached through controlled and targeted nanoscale therapeutics.

In 2005 the National Cancer Institute provided a vision for nanotechnologybased cancer treatment that combines targeted delivery with imaging, diagnostics, and the ability to provide multiple therapies within a single nanoscale construct [5]. The design of such a multifunctional construct is inherently complex as it requires combining different molecular, colloidal, and/or particulate agents that, for example, may have different degrees of hydrophobicity or thermal instability. Furthermore, the construct must be colloidally stable, resist protein adsorption and immune system recognition, and achieve cellular targeting in its native form (i.e. without 'losing' components or cargo during circulation).

Liposome-nanoparticle assemblies (LNAs) represent a promising route for designing multifunctional therapeutic constructs. They draw inspiration from 
magnetoliposomes (liposomes containing encapsulated magnetic nanoparticles [6-11]) and have also been referred to in recent literature as liposome-nanoparticle hybrids or liposome-nanoparticle complexes (Lip-NPs) [12, 13]. LNAs consist of liposomes that contain nanoparticles encapsulated in the aqueous core, embedded in the lipid bilayer, or bound (decorated) onto the surface (Figure 2-1). While liposomes and nanoparticles have both been approved separately for clinical use, the research and development of LNAs is still relatively new. Liposomes are attractive for drug delivery and biomedical imaging because they are biocompatible carriers capable of protecting and transporting hydrophobic and/or hydrophilic therapeutic molecules. Nanoparticles (NPs, up to $100 \mathrm{~nm}$ ) have also been shown to be effective transporters, contrast agents, and agents capable of providing in vivo heating when subjected to external stimuli such as alternating current electromagnetic fields (EMFs; typically at radiofrequencies, RF) or light [14-19]. LNAs can incorporate the intrinsic properties of liposomes and NPs, providing novel multifunctional therapeutic and diagnostic vehicles. This concept was depicted by Pradhan et al. [20] for folate receptor and magnetically targeted magnetoliposomes capable of combined drug delivery and hyperthermia (Figure 2-2). Principle advantages of LNAs include the following:

- Delivery of hydrophobic and hydrophilic molecules and NPs, including small NPs $(<25 \mathrm{~nm})$ that are less prone to endocytic uptake due to the high curvature energy required for a membrane to 'wrap' around the particle [21]. 
- Strategies for processing, stabilizing, and targeting liposomes are well established [22].

- NPs can be magnetically guided for targeting in vivo and provide a triggering mechanism for controlled release (not discussed in detail herein).

- Surface-bound NPs can also enhance the colloidal stability of LNAs and bilayer-embedded NPs can reduce spontaneous leakage [23-26].

The objective of this article is to provide a detailed review of LNA design and structure with an emphasis on recent work that utilize photothermal (via gold NPs) or RF heating (via iron oxide NPs) to achieve hyperthermia treatment, controlled drug release, or combined hyperthermia and drug release. LNAs containing carbon fullerenes such as $\mathrm{C}_{60}$ of $\mathrm{C}_{70}$ (i.e. fullerenosomes, see references [27-36]) are promising therapeutic structures and provide insight into LNA design, but will not be discussed herein. Likewise, NPs containing supported lipid bilayer coatings are also quite promising, but will not be discussed (see, for example [7, 37-39]). A discussion of reported LNA performance in vitro and in vivo will be provided. This compliments a review of "liposome-nanoparticle hybrids" by Al Jamal and Kostarelos in 2007 [12]. Recent reviews focusing on liposomes or NPs for therapeutic application, which are discussed only briefly herein, are provided in references [22, 40-43] and [4, 15-17, 4448], respectively. An expert opinion is provided that focuses on the need for more complete design principles, additional characterization of LNA structure and stability, and the validity of local heating. 

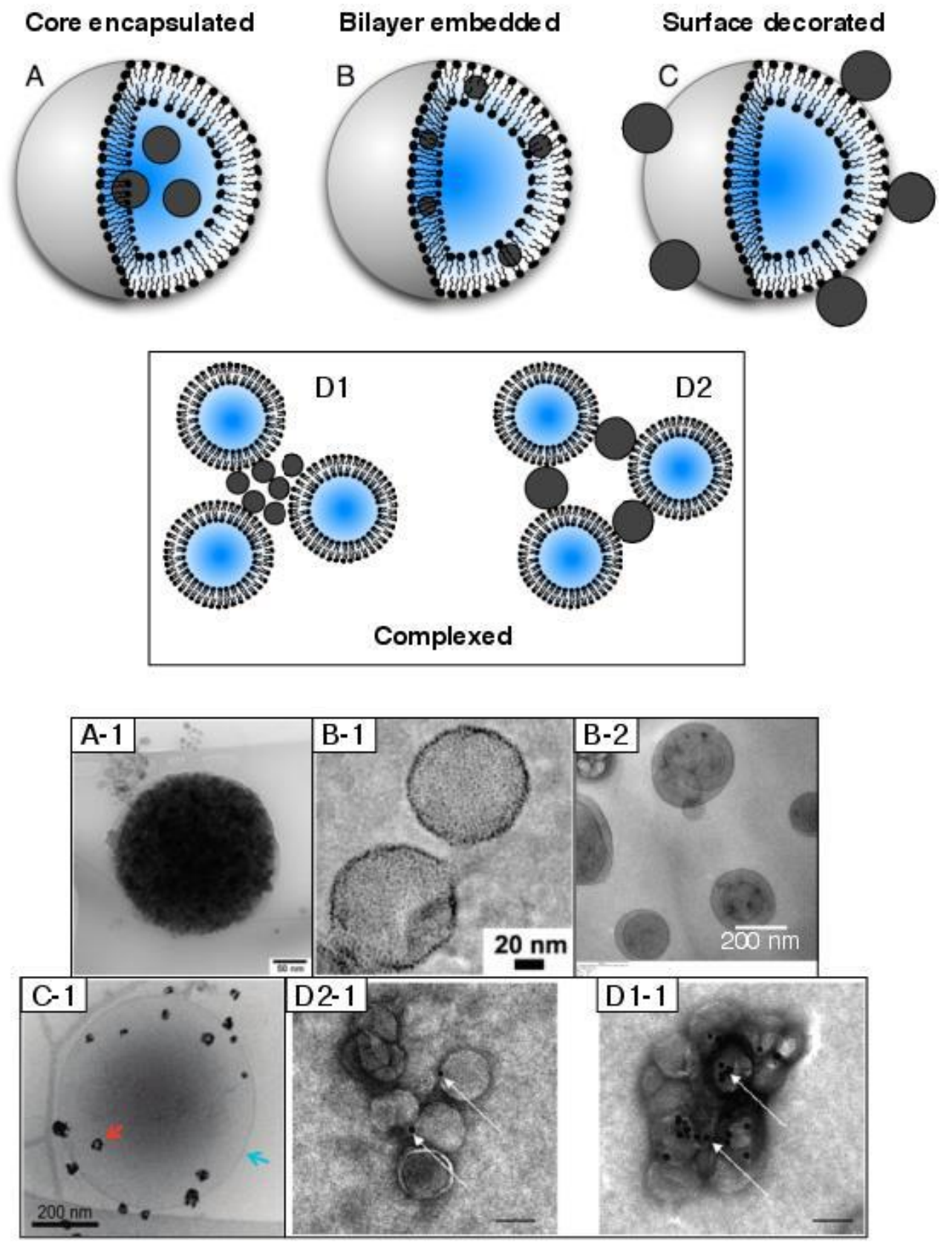

Figure 2-1. Schematics and TEM micrographs of liposome-nanoparticle assemblies.

Schematic and TEM micrographs of LNAs formed by encapsulated hydrophilic nanoparticles with an aqueous liposome core (A; A-1 [70]), embedding hydrophobic nanoparticles within a liposome bilayer (B; B-1 [82], B-2 [23]), or binding hydrophilic nanoparticles to a liposome surface (C; C-1 [92]). Surface decoration (C) can also be used to create controlled aggregates or complexes (D; D1-1, D2-1 [13]). Structures and proportions are not to scale. Reprinted from [13, 23, 70, 82, 92] with permission. 


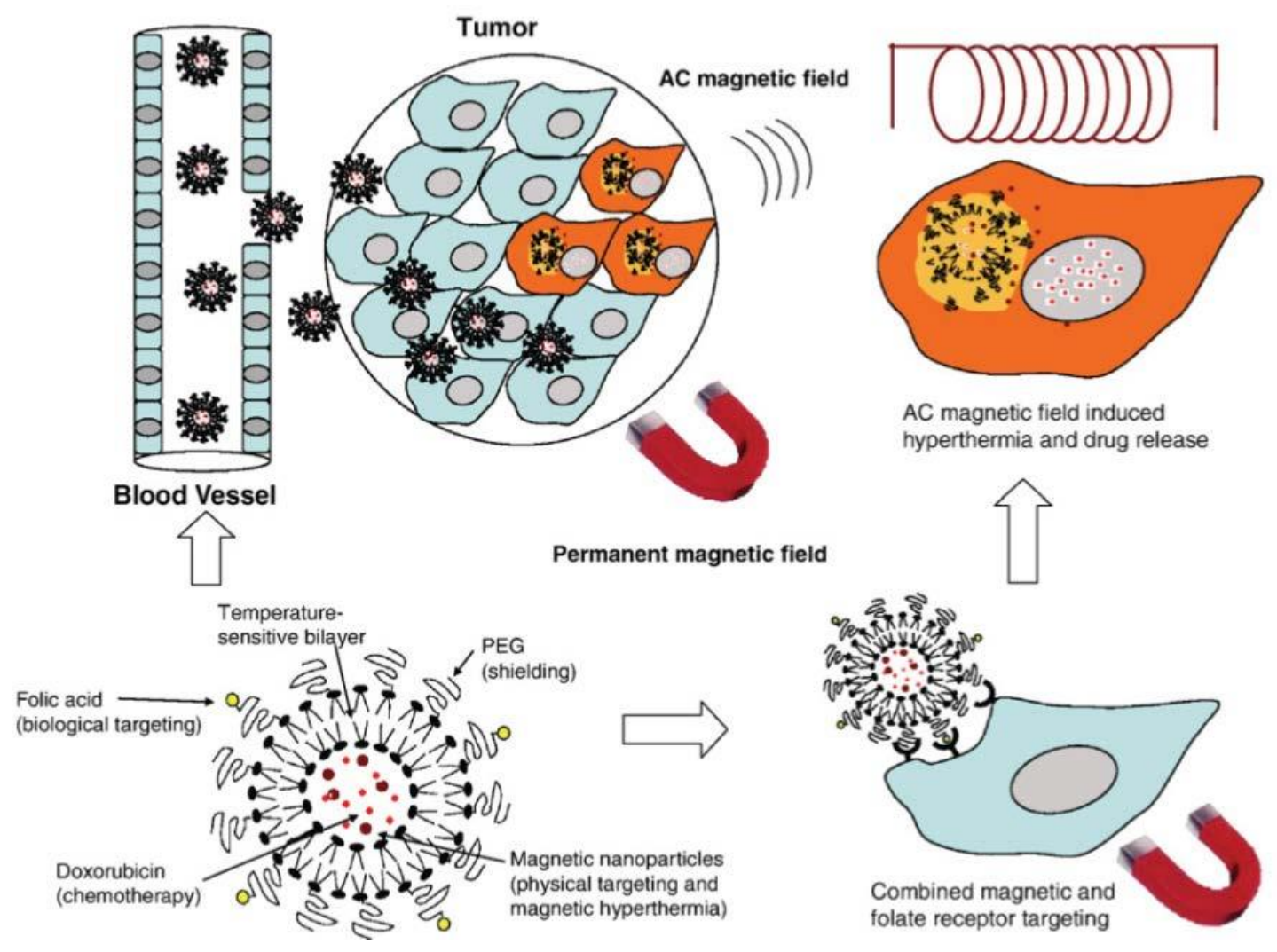

Figure 2-2. Conceptualization of a multifunctional liposome-nanoparticle assembly.

The concept of a multifunctional LNA (a temperature sensitive magnetoliposome containing co-encapsulated iron oxide nanoparticles and doxorubicin) for cancer thermo-chemotherapy from Pradhan et al. [20]. Passive targeting can be achieved through the enhanced permeation and retention (EPR) effect of tumor vasculature, and active targeting can be achieved via folate receptor and by applying a permanent magnetic field. The application of an AC electromagnetic field can be used to release the drug and achieve hyperthermia treatment. Reprinted from [20] with permission.

\subsection{Liposomes}

Since the pioneering work by Bangham and Papahadjopoulos in the 1960s [4951], liposomes have become a well-established platform for administering therapeutic and imaging agents. In 1973, Gregoriadis reported the potential of liposome-aided 
drug delivery and started what would become a burgeoning new field of liposomes as nanoscale delivery vehicles [52]. Since then, liposomes have become one of the most reliable systemic drug delivery systems, particularly because of their biocompatibility and ability to reduce or prevent drug degradation and toxicity.

Liposomes are self-assembled spherical vesicles consisting of one (unilamellar) or multiple (multilamellar) lipid bilayers surrounding an internal aqueous core. Bilayer thickness $\left(l_{\mathrm{b}}\right)$ is $\sim 5 \mathrm{~nm}$ thick $\left(l_{\mathrm{b}}\right)$, of which $3 \mathrm{~nm}$ is the acyl lipid tail region. Liposomes can be prepared with zwitterionic, anionic, or cationic lipids, and the net liposome surface charge can be adjusted by mixing different ratios of these components. Lipids with headgroup-conjugated polyethylene glycol (PEG) and ligands can be used to improve liposome stability, increase blood circulation times, and for cellular targeting is achieved using [22, 41]. For drug delivery and diagnostics, liposomes are attractive because of their ability to encapsulate both hydrophilic (in the aqueous core or bound to the liposome surface) and hydrophobic (in the lipid bilayer) molecules. This enhances the solubility and stability of these molecules and prolongs their bioavailability.

Release of encapsulated molecules from liposomes is controlled by the permeability through the lipid bilayer, which can be achieved by transbilayer diffusion or transient pore formation triggered by bilayer disruption or phase separation. Phase separation can be induced by 'melting' the liposomal bilayers - i.e. heating to a temperature greater than the characteristic main phase transition or melting temperature of the lipids $\left(T_{\mathrm{m}}\right)$. Below $T_{\mathrm{m}}$ the lipids are in the solid or gel phase in 
which the lipids are rigid and highly organized. Above $T_{\mathrm{m}}$ the lipids are disordered in a liquid crystalline or fluid phase. Permeability is high at the interface between gel and fluid phases. Phase separation and bilayer permeability can be manipulated by adjusting the lipid bilayer composition. A simple example illustrating this principle can be made with dipalmitoylphosphatidylcholine (DPPC, $T_{\mathrm{m}}=42{ }^{\circ} \mathrm{C}$ ) and dimyristoylphosphatidylcholine (DMPC, $T_{\mathrm{m}}=23^{\circ} \mathrm{C}$ ). At a DPPC/DMPC molar ratio of $74: 26$ the melting temperature occurs at physiological temperature $\left(37^{\circ} \mathrm{C}\right)$. Furthermore, cholesterol is commonly incorporated into the bilayer to reduce membrane fluidity above the melting temperature. Membrane fluidity is affected by, for example, $\mathrm{pH}$, ion concentration, and the presence of molecules absorbed into the bilayer.

Drug delivery from liposomes is accomplished by cellular uptake, which can occur by adsorption, endocytosis, fusion, and/or lipid transfer [41, 42, 53]. Adsorption is the association of liposome bilayer with cell bilayer without destroying the liposome bilayer or being internalized by the cell. Adsorption can be specific (assisted by targeting ligands such as antibodies) or nonspecific (controlled by intermolecular and surface forces). Endocytosis involves the uptake of liposomes into the cell by encapsulation within endosomes. Release of drugs to the cytoplasm can occurs by membrane destabilization of the encapsulating endosome or by delivery to lysosomes. Lysosomes have an acidic $\mathrm{pH}$ and contain lysing enzymes. Drug release is accomplished when lysosome enzymes hydrolyze the lipid bilayer releasing the drug. Lysosome drug release is only effective when the encapsulated drugs are not 
susceptible to lysosome enzymes and $\mathrm{pH}$. Fusion involves the adsorption and incorporation of the liposome bilayer with the cell membrane, releasing the payload into the cytoplasm. Finally, lipid transfer involves the exchange of lipids between the liposome bilayer and the cell membrane without enveloping the liposome [41, 42].

\subsection{Gold and iron oxide nanoparticles}

Imaging and photothermal effects of gold NPs stem from their enhanced surface plasmon resonance (SPR), where visible or near-infrared light is absorbed causing oscillation of surface electrons [54]. SPR absorbance and the wavelength range are dependent upon nanoparticle size, core/shell configuration (e.g. silica core/gold shell [55]), and geometry. Shifts in these properties are indicative of the degree of NP aggregation and/or molecular adsorption on the NP surface [19]. For photothermal therapy, absorbed light energy is converted into local heat that thermally diffuses into the surrounding medium. Varying NP size and core/shell configuration provides a means of tuning the frequency window for photothermal therapy. It is generally accepted that gold NP-mediated phototherapy is attributed to heat or resulting bubble nucleation depending on the light intensity and mode of exposure [19]. However, recent work by Krpetic et al. [56] at low light energies suggests that photochemical effects - the formation of free radicals during NP irradiation - may play an important role. In addition to photothermal heating, electromagnetic fields operating at RF can also be used to heat gold NPs. For example, Gannon et al. [57] examined the effect of NP concentration and RF field strength on the heating rates of 5 
$\mathrm{nm} \mathrm{Au} \mathrm{NPs} \mathrm{in} \mathrm{water.} \mathrm{A} \mathrm{rate} \mathrm{of} \sim 74{ }^{\circ} \mathrm{C} \min ^{-1}$ was measured using an $800 \mathrm{~W}$ RF field at a NP concentration of $67 \mu \mathrm{M}$.

The magnetic properties of iron oxide NPs, notably single domain superparamagnetic magnetite $\left(\gamma-\mathrm{Fe}_{2} \mathrm{O}_{3}\right)$ or maghemite $\left(\mathrm{Fe}_{3} \mathrm{O}_{4}\right)$, can also be exploited for imaging and therapy. They act as contrast agents for MR imaging, can be directed by static magnetic fields (magnetic drug delivery), and can be heated by RF (hyperthermia) $[16,18,58]$. RF heating is due to magnetic losses being converted to heat, typically at low frequencies between $100-400 \mathrm{kHz}$. The magnetic losses for NPs $<\sim 30 \mathrm{~nm}$ are due to Néel relaxation, arising from rapidly alternating magnetic dipole moments, and Brownian relaxation, arising from nanoparticle rotation and viscous losses (friction). RF heating is advantageous because it is non-invasive, easily penetrates the body, and is physiologically acceptable for up to $1 \mathrm{~h}$ if the product $H f$, where $H$ is the field amplitude (current $\times$ number of coils per length) and $f$ is the frequency, is below $4.85 \times 10^{5} \mathrm{kA} \mathrm{m}^{-1} \mathrm{~s}^{-1}$ [59]. NP heating capability is based on the inherent specific absorbance rate $\left(S A R, \mathrm{~W} \mathrm{~g}^{-1}\right)$ of the nanoparticles

$$
S A R=\frac{c_{p}}{m_{N P}} \frac{\Delta T}{\Delta t}
$$

where $c_{p}$ is the average heat capacity of the sample, $m_{N P}$ is the NP mass, and $\Delta T / \Delta t$ is the initial heating rate of the sample. $S A R$ values up to approximately $700 \mathrm{~W} \mathrm{~g}^{-1}$ can be obtained depending on the NP size, composition, and surface coating [18]. 


\subsection{Nanoparticle-mediated hyperthermia}

NPs capable of RF or photothermal heating have been used for local hyperthermia treatment of malignant tissues, which involves heating the tissues to temperatures between $\sim 40-45{ }^{\circ} \mathrm{C}$ [60]. During hyperthermia, heat denatures intracellular proteins inducing death by necrosis or apoptosis. Hyperthermia has also been shown to make tumor cells more vulnerable to therapies, such as chemotherapy and radiotherapy, therefore it can be used in conjunction with these therapies $[20,61$, 62]. The use of conventional hyperthermia (i.e. without NPs) has been tempered in recent years because of difficulty in applying heat to deeper tumors and delivering targeted heating. This difficulty may be addressed by targeting NPs to malignant cells and tissues.

Heat transfer within tissues via NP heating can be described by a modified Pennes' bio-heat transfer model [63]

$$
\rho_{t} c_{p, t} \frac{\partial T}{\partial t}=\nabla \cdot\left(k_{t} \nabla T\right)+\rho_{b} c_{p, b} \omega_{b}\left(T-T_{b}\right)+Q_{m}+Q_{N P}
$$

where $\rho_{t}$ is the tissue density and $c_{p, t}$ is the tissue heat capacity. The first term on the right hand side (RHS) of the equation describes the conductive heat transfer $\left(k_{t}\right.$ is the tissue thermal conductivity) and the second term describes the convective heat transfer ( $\rho_{b}$ is the blood density, $c_{p, b}$ is the blood heat capacity, $\omega_{b}$ is the blood perfusion rate, and $T_{b}$ is the blood temperature). $Q_{m}$ is the rate of heat generated metabolically and $Q_{N P}$ is the rate of heat generated from the power dissipation by the NPs, which accounts for the concentration of NPs (e.g. $Q_{N P}$ represented as $S A R$ ). Equation 2 
represents the case where the temperature profile in a tissue mass (macro-scale) can be determined as a function of NP concentration and applied field strength (e.g. laser or RF). It has been shown theoretically that sufficient iron oxide NP heating can be achieved $\left(>42{ }^{\circ} \mathrm{C}\right)$ at low blood perfusion rates to achieve tissue-level hyperthermia [64]. For cellular-level heating (nano- or micro-scale), convective heat transfer due to blood transfusion and $Q_{m}$ are eliminated from equation 2, yielding the expression reported by Keblinski et al. [65] for RF NP heating and $\mathrm{Xu}$ et al. [66] for $\mathrm{Fe}_{3} \mathrm{O}_{4} \mathrm{NP}$ hyperthermia in vitro.

\section{LNA Formation and Structure}

LNA design strategies include the encapsulation of individual or multiple NPs within the aqueous core of the liposome, embedding hydrophobic NPs in the lipid bilayer, and binding or conjugating NPs to the liposome surface (Figure 2-1). Tables 2-1 and 2-2 contains a list of Au and iron oxide LNAs reported in the literature since 2008. For a given design strategy, the functionality of a LNA is determined by the liposome composition, the type of NPs employed, the intermolecular and surface interactions between the lipid bilayer and NP, and (as in all cases) the colloidal stability. LNAs can be used to concentrate NPs and shield them from the adsorption of exogenous molecules. Concentrating the NPs can increase the degree of intracellular delivery, which is critical, for example, in imaging and hyperthermia applications. In turn, shielding the NPs from the adsorption of biomolecules can enhance their bioavailability and reduce the need for more complex NP surface chemistries. The 
caveat here, which is germane to all LNA configurations, is that the liposome itself must contain functional lipids or surface coatings for stabilization and, when needed, targeting [12].

In addition to serving as a vehicle for NP delivery and being multifunctional, LNAs can be used to overcome design challenges of 'conventional' liposomes. With respect to delivery, the main challenge includes creating an assembly that is stable and retains its cargo during both storage and circulation, but is capable of releasing its cargo in vivo at a target site (i.e. stable until it needs to become unstable). This challenge has been addressed by using lipid mixtures that melt near physiological temperature or through chemical mechanisms such as pH-sensitive lipids; which has ultimately reduced the number of viable lipid molecules that can be used. In contrast, LNAs can utilize physical triggers, predominantly NP heating, to control the onset and duration over which a molecule is released. While lipid composition plays an active role in determining the release profile from LNAs, the lipids themselves would not provide the release trigger. This could greatly expand the range of lipids amenable to liposomal release [12].

Finally, LNAs can potentially be used to deliver high concentrations of NPs capable of RF or photothermal heating for local hyperthermia. Targeted LNA administration can be achieved through known liposomal-based mechanisms (e.g. targeting lipids) and may provide a local heat source for both hyperthermia and drug release without adversely effecting adjacent tissue. 
Table 2-1. Reported LNAs based on Au NPs since 2008.

\begin{tabular}{|c|c|c|c|c|c|c|}
\hline Lipids (ratio) ${ }^{a}$ & $\begin{array}{l}\text { Liposome } \\
\text { charge }\end{array}$ & $\begin{array}{c}\text { NP } \\
\text { Diameter } \\
(\mathbf{n m}) \\
\end{array}$ & Surface Coating & Lipid:NPa & Association & Ref. \\
\hline DOPC:DOTAP (8:2) & Cationic & 80 & Citrate & n.r. ${ }^{b}$ & Encapsulation & [91] \\
\hline DPPC:DSPC (9:1) & Zwitterionic & 2.5 & Hexanethiol & $\begin{array}{l}17.2: 1 \\
(\mathrm{w} / \mathrm{w}) \\
\end{array}$ & Embedment & [95] \\
\hline EggPC & Zwitterionic & 2 & Dodecanethiol & $\begin{array}{l}100: 1- \\
1500: 1\end{array}$ & Embedment & [82] \\
\hline $\begin{array}{l}\text { DPPC:DSPC } \\
(9: 1)\end{array}$ & Zwitterionic & 4 & Mercaptosuccinic acid & $10: 1(w / w)$ & Encapsulation & [95] \\
\hline DPPC:DSPC (9:1) & Zwitterionic & 1.4 & DPPE-Nanogold ${ }^{\mathrm{TM}}$ & n.r. & $\begin{array}{l}\text { Embedment, } \\
\text { Decoration }\end{array}$ & {$[24]$} \\
\hline $\begin{array}{l}\text { DOPC } \\
\text { DOPC:DOPC }+(90: 10) \\
\text { DOPC:DOPP }(9: 1)\end{array}$ & $\begin{array}{l}\text { Zwitterionic } \\
\text { Cationic } \\
\text { Anionic }\end{array}$ & n.r. & Ascorbic acid & n.r & Decoration & {$[87]$} \\
\hline $\begin{array}{l}\text { EYPC } \\
\text { EYPC:DDAB }(9: 1) \\
\text { EYPC:PEG-DSPE }(95: 5)\end{array}$ & $\begin{array}{l}\text { Zwitterionic } \\
\text { Cationic } \\
\text { Zwitterionic }\end{array}$ & 13 & Citrate & $10: 1,1: 1$ & Decoration & [88] \\
\hline $\begin{array}{l}\text { EggPC:DOTAP } \\
(9: 1 \mathrm{w} / \mathrm{w})\end{array}$ & Cationic & 4 & $\begin{array}{l}\text { Mercaptopropionic } \\
\text { acid }\end{array}$ & $\geq 3.6 \times 10^{-3}: 1$ & Decoration & [89] \\
\hline $\begin{array}{l}\text { DPPC:DPTAP:Chol } \\
(6: 3: 1 \mathrm{w} / \mathrm{w})\end{array}$ & Cationic & 20 & n.r. & & Complexation & [13] \\
\hline DPPC & Zwitterionic & 33 & PEG & $\begin{array}{l}1.8: 1 \\
(\mathrm{w} / \mathrm{mol}) \\
1: 1(\mathrm{w} / \mathrm{mol})\end{array}$ & $\begin{array}{l}\text { Encapsulation } \\
\text { Embedment, } \\
\text { Decoration }\end{array}$ & [92] \\
\hline
\end{tabular}

${ }^{a}$ molar ratios provided unless noted otherwise.

bestimated at 4 liposomes per NP. 
Table 2-2. Reported LNAs based on iron oxide NPs since 2008.

\begin{tabular}{|c|c|c|c|c|c|c|}
\hline Lipids (ratio) ${ }^{a}$ & $\begin{array}{l}\text { Liposome } \\
\text { charge }\end{array}$ & $\begin{array}{c}\text { NP } \\
\text { diameter } \\
(\mathbf{n m}) \\
\end{array}$ & $\begin{array}{l}\text { Surface } \\
\text { coating }\end{array}$ & Lipid:NPa & Association & Ref. \\
\hline \multicolumn{7}{|c|}{ Magnetite $\left(\mathrm{Fe}_{3} \mathrm{O}_{4}\right)$} \\
\hline DPPC:Chol (75:25) & Zwitterionic & 12.5 & n.d. & $0.8: 1$ & Encapsulation & {$[98]$} \\
\hline $\begin{array}{l}\text { [maleimide]PEG-DSPE:FAM-DOPE } \\
(10: 1 \mathrm{w} / \mathrm{w})\end{array}$ & & $10-14$ & $\begin{array}{l}\text { Heptanioc acid, } \\
\text { acetic acid }\end{array}$ & $1: 1.8(\mathrm{w} / \mathrm{w})$ & Encapsulation & [99] \\
\hline $\begin{array}{l}\text { DMPC:Chol:XL }{ }^{\mathrm{b}}(47.5: 47.5: 5) \\
\text { DPPC:DMPC:XL }(9.5: 85.5: 5)\end{array}$ & Zwitterionic & 10 & Catechol & $\geq 8.3: 1(\mathrm{~mol} / \mathrm{w})$ & Complexation & {$[90]$} \\
\hline \multicolumn{7}{|c|}{ Maghemite $\left(\mathrm{g}-\mathrm{Fe}_{2} \mathrm{O}_{3}\right)$} \\
\hline DPPC:Chol (67:33) & Zwitterionic & 10 & Glutamic acid & n.r. & Encapsulation & [100] \\
\hline $\begin{array}{l}\text { DPPC:Chol }(5: 1,15: 3 \mathrm{w} / \mathrm{w}) \\
\text { DPPC:DSPC:Chol }(10: 5: 3 \mathrm{w} / \mathrm{w})\end{array}$ & Zwitterionic & 43 & Dextran & n.r. & Encapsulation & {$[93]$} \\
\hline DPPC & Zwitterionic & 5 & Oleic acid & $\begin{array}{l}1000: 1- \\
10000: 1\end{array}$ & Embedment & {$[23]$} \\
\hline
\end{tabular}

${ }^{a}$ molar ratios provided unless noted otherwise.

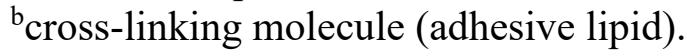




\subsection{Core encapsulation}

Encapsulating inorganic NPs within the aqueous core of liposomes is one of the simplest and earliest developed LNA configurations (e.g. magnetoliposomes or MLs [7, 9]). They can be prepared by encapsulating preformed NPs in solution or by forming NPs within the liposome core as first shown by Papahadjopoulos in 1983 [67]. The later approach will not be discussed herein. Encapsualted LNAs (e-LNAs) can be prepared by thin film hydration (TFH), double emulsion (DE) [68], or reverse phase evaporation (REV) [69]. Prior to removing unencapsulated NPs or diluting, post-formation liposome processing such as membrane extrusion or sonication can be employed. The obvious design constraints are that the nanoparticles must be colloidal stable during LNA formation and that their diameter $(d)$ must be less than that of the aqueous liposome core. When $d_{\text {core }}=d_{\mathrm{NP}}$ these structures are referred to as supported lipid bilayers (SLBs; i.e. NPs containing a lipid bilayer coating). Based on close packing of spheres and $d_{\mathrm{core}} \gg d_{\mathrm{NP}}$, the maximum theoretical number of encapsulated NPs is $n \approx 0.74\left(V_{\text {core }} / V_{\mathrm{NP}}\right)$ where $V$ represents the volume of the core or NP. Wijaya and Hamad-Schifferli [70] have shown that it is possible to approach this limit, demonstrating high-density encapsulation of $\mathrm{Fe}_{3} \mathrm{O}_{4} \mathrm{NPs}\left(d_{\mathrm{NP}}=12.5 \mathrm{~nm}\right)$ within DPPC liposomes (Figure 2-1, A-1). With this design the available core volume for coencapsulating aqueous drug molecules decreases within increasing NP concentration. However, the ability for embedding hydrophobic molecules within the bilayer is unaffected by NP concentration. 
The structure of e-LNAs is dependent on the osmotic pressure differential across the lipid bilayer, and the attractive or repulsive forces between the bilayer and the NPs. The elasticity of the bilayer determines how the LNA will deform in response to these forces. Attractive forces can include van der Waals, hydrophobic, and electrostatic interactions; and repulsive forces can include electrostatic, depletion, hydration, and steric interactions. As classically described by Lipowsky and Döbereiner [71], adhering and non-adhering nanoparticles can lead to changes in bilayer curvature, which can impact liposome size, shape, and phase homogeneity. This occurs when different particles are present within (i.e. encapsulated NPs) and outside (e.g. sugars or proteins) liposomes. For non-adhering encapsulated particles, the bilayer can curve towards the larger particles. In contrast, for small adhering encapsulated particles (attractive) where $d_{\mathrm{core}}>d_{\mathrm{NP}}$ and $d_{\mathrm{NP}}<2 l_{\mathrm{b}}$ the bilayer can curve away from the particles. For large adhering particles where $d_{\mathrm{NP}}>>2 l_{\mathrm{b}}$ the bilayer can curve around or engulf the particles. Given that NP adhesion to bilayers can significantly alter LNA structure and morphology, LNAs with encapsulated NPs are generally formed with small non-adhering NPs. The exception to this is LNAs formed by coating a single large NP with an adsorbed or supported lipid bilayer (not discussed herein).

Pradhan et al. [72] compared the encapsulation efficiency of $10 \mathrm{~nm} \mathrm{Fe}_{3} \mathrm{O}_{4} \mathrm{NPs}$ coated with lauric acid in LNAs (or more specifically MLs) composed of egg PC:cholesterol (1:0 to 1:2, molar ratio) and formed by THF and DE. This represents a non-adhesive system. In general higher encapsulation efficiency was achieved by THF 
compared to DE. This was attributed to NP aggregation due to lauric acid stripping from the nanoparticle surfaces during the DE process. In both cases, the observation that an Egg PC:cholesterol ratio of 2:1 yielded the best encapsulation efficiency (70\% via THF) was attributed to cholesterol inducing a single liquid ordered bilayer phase. Changes in liposome size upon encapsulation were not reported.

In similar work, Sabate et al. [73] examined the effect of $\mathrm{Fe}_{3} \mathrm{O}_{4} \mathrm{NP}$ concentration coated with tetramethylammonium hydroxide (58 $\mathrm{nm}$ hydrodynamic $d \mathrm{NP}$ ) on the encapsulation efficiency of extruded soybean PC MLs. This represents an adhesive system. The encapsulation efficiency decreased from $96.6 \%$ at $1.22 \mathrm{~g}$ $\mathrm{Fe}_{3} \mathrm{O}_{4} / \mathrm{mol} \mathrm{PC}$ to $18.5 \%$ at $119.95 \mathrm{~g} \mathrm{Fe}_{3} \mathrm{O}_{4} / \mathrm{mol} \mathrm{PC}$. This was attributed to electrostatic interactions (attraction) between the cationic NPs and the PC bilayers. The size of the MLs increased from 140 to $197 \mathrm{~nm}$, consistent with lower curvature due to NP adhesion at the inner bilayer surface.

Gomes et al. [74] prepared polyelectrolyte-coated MLs by encapsulating $8 \mathrm{~nm}$ anionic $\gamma-\mathrm{Fe}_{2} \mathrm{O}_{3}$ NPs within egg PC liposomes and then coating with alternating poly(allylamine hydrochloride) and poly(sodium 4-styrenesulfonate) layers. The final coating determined the surface charge (anionic PSS or cationic PAH). The size ranged from $200-400 \mathrm{~nm}$ and the polyelectrolyte coating stabilized them against detergentinduced leakage, which is caused by membrane disruption or solubilization. 


\subsection{Bilayer embedment}

Embedding NPs into the bilayer requires that the NPs be hydrophobic and have diameters comparable to or smaller than the thickness of the lipid bilayer $(\sim 5 \mathrm{~nm}$;

Figure 2-1, B). LNAs formed by bilayer embedment (b-LNAs) can be advantageous as many nanoparticles are inherently hydrophobic or synthesized in organic solvents (e.g. in reverse microemulsions where the surfactant is the initial surface coating) before undergoing surface modification for aqueous environments. Similar to the ability of cells to accommodate membrane proteins, liposomes can distort to accommodate hydrophobic NPs that exceed the thickness of hydrophobic acyl region of the bilayer ( 3 nm) [23, 75-77]. As with proteins, embedded NPs can affect lipid packing, lipid phase behavior, transbilayer permeability, and LNA structure and morphology [23, 28, $34,76,78-82]$. A unique aspect of b-LNAs (as well as surface decorated LNAs) is that the NPs can provide direct localized heating to the bilayer in the presence of external stimuli to trigger release $[23,24]$.

It is intuitive that the size of a NP (core + surface coating) and its concentration, or more specifically the lipid:NP ratio, will influence how the lipid bilayer distorts to accommodate it and the resulting LNA structure (Figure 2-3). Theoretical studies by Ginzburg and Balijepalli [83] and Wi et al. [84] suggest that the maximum size of a NP $(d \mathrm{NP})$ that can be incorporated into a LNA while maintaining $a$ lipid bilayer structure is ca. $6.5 \mathrm{~nm}$ (Figure 2-3, A and B). Above this size micellar structures are more energetically favorable due to high local curvature strain within the bilayer [84]. Experimental verification of this critical size and, furthermore, the 
general size effects of NPs on embedment mechanism and LNA structure are more elusive.

Clustering of embedded NPs has been observed by Rasch et al. [82] in LNAs with dodecanethiol-coated $\mathrm{Au}\left(d_{\mathrm{NP}}=1.6-1.8 \mathrm{~nm}\right)$ (Figure 2-1, B-1; Figure 2-3, A). They showed that high NP loading with uniform distribution can be achieved in PC liposomes via thin film hydration (with sonication and extrusion). Janus particles can be prepared with embedded NPs clustered in approximately one half of the liposomes via detergent loading followed by dialysis. Clustering occurs as the liposomes minimize the energy penalty for bilayer deformation - i.e. for a given concentration of embedded NPs the periodic bilayer bending energy needed to accommodate individual particles is greater than that needed to accommodate nanoparticle clusters. Park et al. [80] and Chen et al. [23] have observed a similar clustering phenomenon with stearylamine-coated 3-4 $\mathrm{nm}$ Au and oleic acid-coated $5 \mathrm{~nm} \gamma-\mathrm{Fe}_{2} \mathrm{O}_{3} \mathrm{NPs}$ in DPPC liposomes (Figure 2-1, B-2), respectively. This suggests that NP clustering is not restricted to $d_{\mathrm{NP}}<2 \mathrm{~nm}[23]$.

In addition to clustering, embedded NPs with $d_{\mathrm{NP}}=2-6.5 \mathrm{~nm}$ can reside in bilayer 'pockets' within individual (Figure 2-3B) or neighboring (Figure 2-3, C) LNAs. These cases arise when the lipid:NP ratio is high ( 1000:1 or greater). This has been observed by Al Jamal et al. [85] for $4 \mathrm{~nm}$ hydrophobic CdSe/ZnS core/shell quantum dots in DOPC bilayers. 


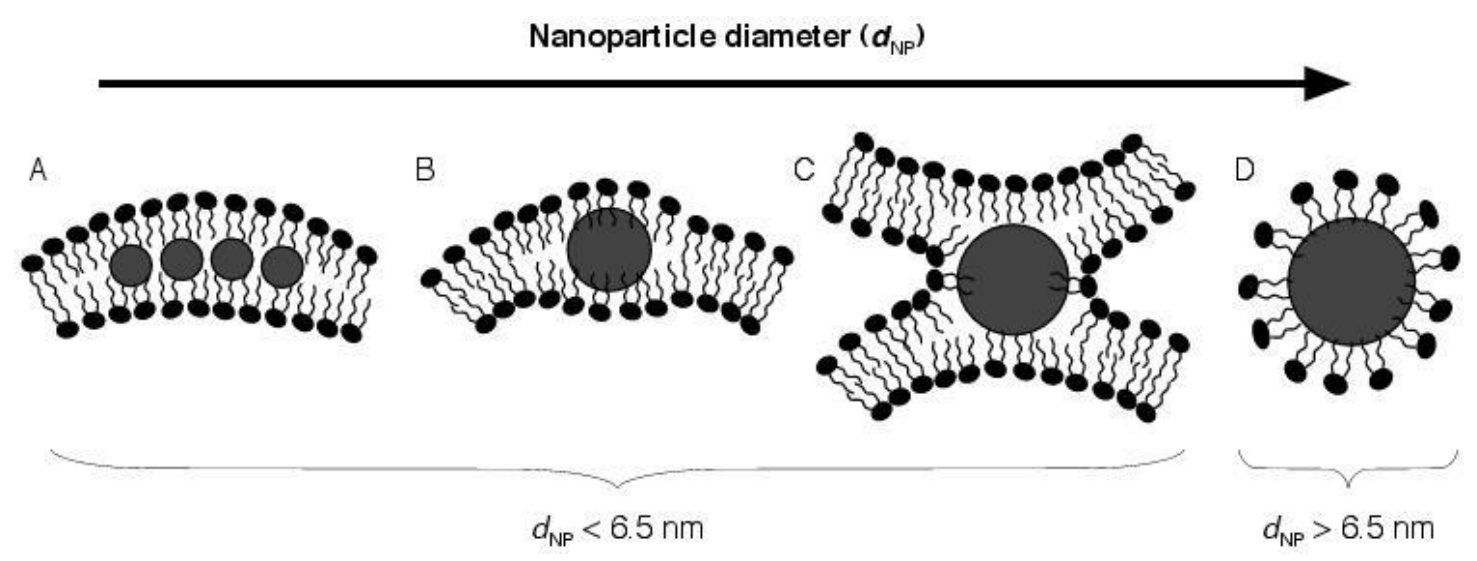

Figure 2-3. Change in bilayer embedment as a function of changing nanoparticle size.

Changes in bilayer embedment mechanism with increasing nanoparticle diameter (dNP, particle core + surface coating). (A) Small nanoparticles (defined herein as dNP $<2 \mathrm{~nm}$ ) cluster together to minimize bilayer bending energy [82]. (B) Larger nanoparticles (defined herein as $\mathrm{dNP}=2-6.5 \mathrm{~nm}$ ) create 'pockets' within the bilayer or (C) bridge adjoining liposomes $[23,77]$. Above $\mathrm{dNP} \approx 6.5 \mathrm{~nm}$, micellization is more energetically favorable than bilayer embedment [84].

\subsection{Surface decoration and complexation}

Decorated LNAs (d-LNAs) are formed when hydrophilic NPs are absorbed onto or coupled to the outer or inner surface of the lipid bilayer (Figure 2-1, C). This is achieved through attractive surface interactions, notably long-range electrostatic attraction. An advantage of d-LNAs is the ease in which they can be prepared - adding NPs to pre-existing liposome dispersions. Similar to bilayer embedment, decorated bilayers also provide direct heating to the bilayer in the presence of external stimuli. The design constraint for forming d-LNAs is dependent on bilayer NP adhesion and curvature. NPs with $d_{\mathrm{NP}}>\sim 20 \mathrm{~nm}$ lead to the formation of SLBs due to liposome adsorption and rupture, followed by the bilayer curving around the particle. The 
critical NP diameter under which d-LNAs can be formed is $d_{\mathrm{NP}}<2\left(k_{\mathrm{b}} / w\right)^{1 / 2}$, where $k_{\mathrm{b}}$ is the bilayer bending elasticity, which is dependent on lipid composition and phase state, and $w$ is the adhesion energy.

The Granick group has shown that stable d-LNA dispersions can be formed using zwitterionic liposomes with decorated cationic or anionic NPs $(<20 \mathrm{~nm})$ with a NP surface coverage above $\sim 25 \%[25,26]$. This was achieved by electrostatic attraction. Lower surface coverage led to aggregation, which demonstrates the need to balance the lipid:NP ratio. It was shown that upon binding the nanoparticles could restructure the lipid bilayer, inducing gel phases in fluid liposomes and fluid phases in gel liposomes [86]. This observation shows that, even without external stimuli, bound NPs can induce changes in lipid phase behavior and, presumably, permeability.

Sau et al. [87] have also used electrostatic binding to prepare d-LNAs with Au NPs. High NP surface coverage was achieved by using anionic Au NPs with physisorbed ascorbic acid and cationic liposomes (9:1 DOPC to ethyl-DOPC; $T_{\mathrm{m}}=-$ $20^{\circ} \mathrm{C}$ ). This high surface coverage was accompanied by NP aggregation due to the high local concentration and (likely) to charge screening via cationic lipids between bound particles (similar to Kojima et al. [88]). Binding was also achieved on zwitterionic and anionic liposomes with decreasing coverage (and NP aggregation), respectively. Pornpattananangkul et al. [89] have taken this one step further and have shown that $\mathrm{pH}$ can be used to control carboxyl-modified (anionic) Au NP binding to cationic liposomes and, in turn, liposome stability. Above the pKa of the carboxyl 
groups the bound NPs stabilize the d-LNAs and prevent aggregation and fusion, while below the pKa the NPs detach and liposome fusion resumes.

Last, LNAs can be formed by complexation (c-LNAs) if the liposomes surround NP aggregates (Figure 2-1, D1 and D2) or the NPs bind to multiple liposomes and act as "bridges" (Figure 2-1, D2). Voldokin et al. [13] have shown that either structure can be formed from the same anionic Au NP-cationic liposome by manipulating electrostatic interactions using via salt concentration. High $\mathrm{NaCl}$ concentration (75 mM) enhanced NP aggregation (Figure 2-1, D1-1) and low salt concentrations inhibited it (Figure 2-1, D2-1). In addition to non-specific physical interactions (electrostatic), cross-linking can be used to create c-LNAs. Mart et al. [90] used $\mathrm{Fe}_{3} \mathrm{O}_{4}$ NPs coated with histidine groups to bind to and complex zwitterionic/cholesterol liposomes containing $\mathrm{Cu}$ (iminodiacetate)-functionalized lipid. The objective was to demonstrate a potential method using histidine-Cu(IDA) binding to form c-LNAs, thereby concentrating a therapeutic and an imaging agents at a target site. The resulting aggregates ranged from 20-100 $\mu \mathrm{m}$ in diameter.

\section{LNA Controlled Release}

This section reviews recent work on gold or iron oxide NP-mediated release from LNAs. Articles that apply these principles in vitro or in vivo are presented in section 4 . 


\subsection{Gold nanoparticles and photothermal effects}

Utilizing the photothermal heating of Au NPs, Paasonen et al. [24] demonstrated the ability to control the release of calcein (622.6 MW) from Au LNAs composed of DPPC/DSPC at 9:1 $\left(T_{\mathrm{m}}=44.9^{\circ} \mathrm{C}\right)$ with e-, $\mathrm{d}-$, and b-LNAs (Figure 2-1, A-C). Leakage was examined with and without UV light at a wavelength of $250 \mathrm{~nm}$ over 30 min at $37^{\circ} \mathrm{C}$. Without UV exposure, spontaneous calcein release was observed for e-LNAs with encapsulated mercaptosuccinic acid-coated NPs and bLNAs with embedded hexanethiol-coated NPs. This was attributed to NP-lipid interactions at the bilayer/water interface and within the acyl tail region, respectively, which reduced bilayer integrity. With UV exposure, direct contact between NPs and the liposomes via bilayer-embedment led to the greatest release ( 90\% at $30 \mathrm{~min}$ ). Intuitively, direct contact would improve the local heat transfer from the NPs to the liposomal bilayers relative to encapsulation. This led to a gel-fluid phase transition where calcein release was presumably enhanced by diffusion at the interface between coexisting gel and fluid domains.

Volodkin et al. [13] demonstrated the release of 5(6)-carboxyfluorescein (CF; 376.3 MW) from LNAs formed by the complexation of $128 \mathrm{~nm}$ cationic liposomes (DPPC/DPTAP/chol, $T_{\mathrm{m}} \sim 40-45^{\circ} \mathrm{C}$ ) and $20 \mathrm{~nm}$ anionic Au NPs. Low $\mathrm{NaCl}$ concentration (7.5 mM) yielded LNAs with NP-mediated liposome bridges (Figure 21, D2-1; type I) and high $\mathrm{NaCl}$ concentration $(75 \mathrm{mM})$ yielded LNAs with liposomecoated NP aggregates (Figure 2-1, D1-1; type II). The type II LNAs were $\sim 5 \mu \mathrm{m}$. CF release from type II LNAs was observed within $5 \mathrm{~s}$ after near-IR irradiation. 
Anderson et al. [91] utilized the principle of plasmonic nanobubble (PNB) formation to control the release of 104 and $240 \mathrm{kDa}$ proteins from cationic LNAs $(\sim 1$ $\mu \mathrm{m})$ containing encapsulated $80 \mathrm{~nm}$ anionic Au NPs. Irradiation was achieved using a single pump laser at $532 \mathrm{~nm}$ over $0.5 \mathrm{~ns}$. Local vapor bubble formation led to mechanical disruption, as opposed to thermal, of the LNA bilayer and rapid protein release. The advantage of PNB formation is heating is isolated within the LNA triggering the immediate release of all encapsulated cargo.

Wu et al. [92] used hollow gold nanoshells (HGNs) encapsulated within or decorating the surface of DPPC liposomes to trigger CF release by near-IR pulses (800 $\mathrm{nm}$ ) via microbubble formation and collapse (Figure 2-1, C-1). LNA release was dependent on the proximity of the HGNs to the liposomes (decorated or tethered HGNs yielded the greatest response) and the laser power. Their results strongly suggest that release was attributed to transient disruption or poration of the lipid bilayer via transient bubble cavitation.

\subsection{Iron oxide nanoparticles and alternating magnetic field effects}

Tai et al. [93] examined CF release from thermosensitive zwitterionic liposomes containing encapsulated dextran-coated $43 \mathrm{~nm} \gamma-\mathrm{Fe}_{2} \mathrm{O}_{3} \mathrm{NPs}_{\text {(Resovist }}{ }^{\mathrm{TM}}$ ) using a high frequency generator $(6.4 \mathrm{~kW}, 750-1150 \mathrm{kHz})$ operating for 5-25 min. CF release from DPPC:Chol (5:1) liposomes without encapsulated NPs was initiated between $35^{\circ} \mathrm{C}$ and $37^{\circ} \mathrm{C}$. In contrast, the LNA analogs exhibited initial release at 34 ${ }^{\circ} \mathrm{C}$ and $32{ }^{\circ} \mathrm{C}$ with 7 and $14 \mathrm{mg} \mathrm{Fe} / \mathrm{ml}$, respectively. This initial release temperature 
was further tuned by increasing the cholesterol content (DPPC:Chol at 15:3) and incorporating a higher melting lipid (DSPC, $T_{\mathrm{m}}=55^{\circ} \mathrm{C}$ ). Release was attributed to NP heating. Using a rat model, they demonstrated that release could also be achieved in vivo.

Chen et al. [23] have recently examined the release of CF from LNAs formed with DPPC and bilayer-embedded oleic acid-coated $5 \mathrm{~nm} \gamma-\mathrm{Fe}_{2} \mathrm{O}_{3} \mathrm{NPs}$ at lipid:NP ratios of 10000:1, 5000:1, and 1000:1 as a function of RF energy (1 kW; 50-250 A, $281 \mathrm{kHz}$ ) (Figure 2-4). Experiments were conducted at non-invasive RF energies near or below $4.85 \times 10^{5} \mathrm{kA} \mathrm{m}^{-1} \mathrm{~s}^{-1}$ for $0-40 \min$ [59]. The greatest release rate was observed at 5000:1, indicating an optimal NP loading for triggering release. This optimum reflected a balance between NP loading and LNA structure - high loading is needed for triggering bilayer release, but can lead to NP aggregation and can compromise LNA structure and stability. A unique observation was the fact that increasing NP loading reduced or eliminated spontaneous leakage by increasing bilayer stability. CF release was attributed to bilayer disruption via local heating and/or LNA rupture, which produced transient voids or pores. 

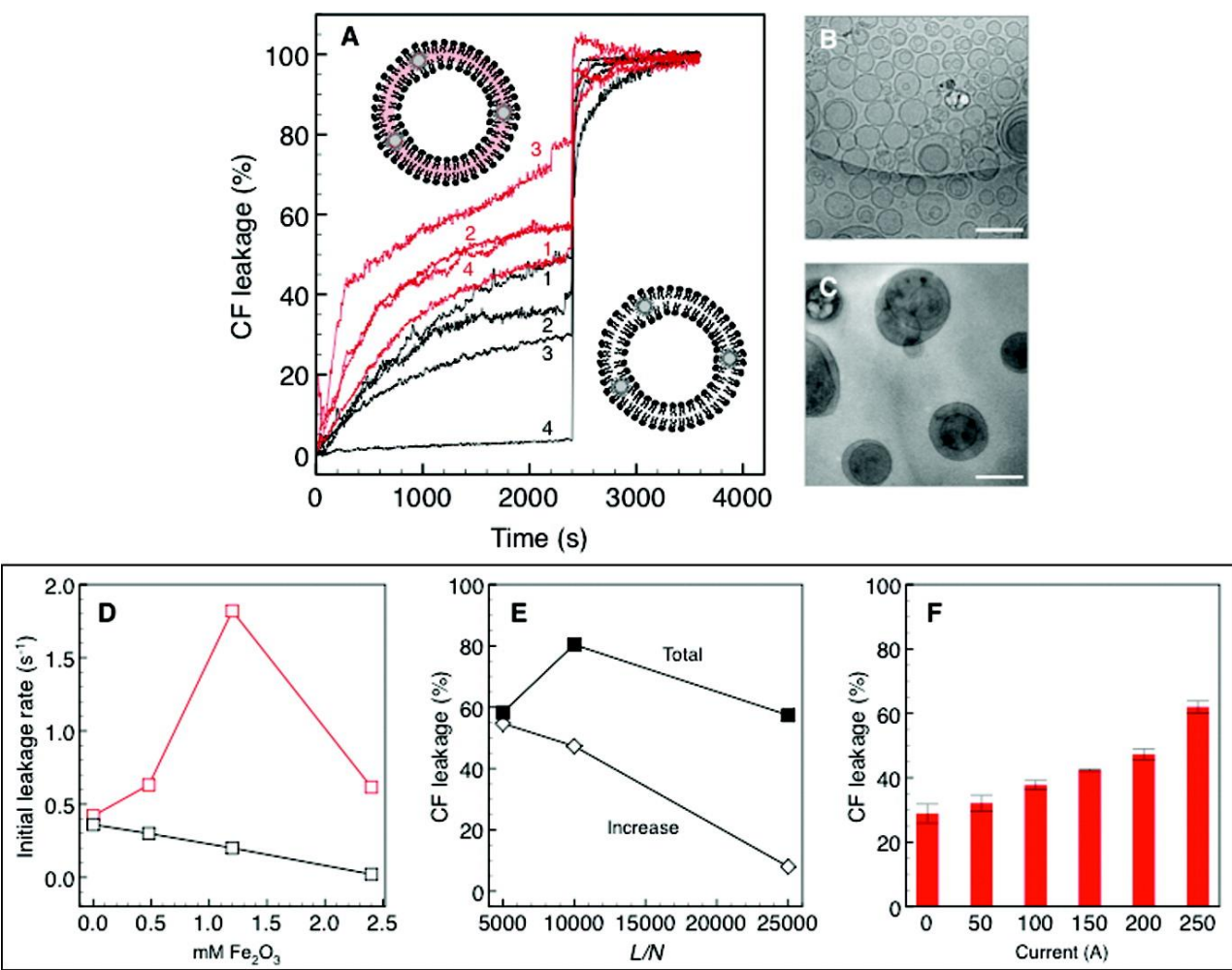

Figure 2-4. Controlled release from a decorated liposome-nanoparticle assembly under the influence of an alternating current electromagnetic field at radio frequency.

Controlled release of carboxyfluorescein (CF), a model encapsulated drug molecule, from magnetoliposomes consisting of $5 \mathrm{~nm}$ hydrophobic iron oxide NPs embedded within the bilayers of DPPC liposomes. (A) Release is shown with (red) and without (black) RF heating at $4.85 \times 10^{5} \mathrm{kA} \mathrm{m}^{-1} \mathrm{~s}^{-1}$ as a function of RF exposure. Images (B) and $(\mathrm{C})$ show bare DPPC liposomes and the magnetoliposomes, respectively. The initial and total leakage is shown as (D) a function of iron oxide concentration and (E) the lipid to NP ratio $(L / N)$, respectively. (F) Total CF leakage after 40 min of RF exposure could be varied with line current. Reprinted from [23] with permission.

\section{LNA Performance In vitro and In vivo}

\subsection{Cellular uptake and drug delivery}

Chithrani et al. [94] prepared anionic Au decorated liposomes with $105 \mathrm{~nm} d \mathrm{~h}$ by incorporating Au-conjugated DPPE (DPPE-Nanogold; $1.4 \mathrm{~nm}$ Au particles) into 
preformed DPPC/cholesterol liposomes. Incorporation of DPPE-Nanogold was confirmed by TEM and EDS. Liposome uptake by HeLa cells in vitro was independent of the DPPE-Nanogold concentration (2000:1, 1000:1, and 500:1 Au NPs per liposome). This key discovery suggests that the presence of DPPE-Nanogold does not influence cell uptake and that high nanoparticle loadings can be achieved in such LNAs without compromising internalization. Examining the intracellular fate revealed that the Au-liposomes were present in lysosomes and accumulated near the nuclear membrane after incubating for $45 \mathrm{~min}$.

Paasonen et al. [95] examined uptake and light-induced calcein release of bLNAs prepared hexanethiol-coated Au NPs (extension of [24]). The LNAs were composed of DSPC:DPPC (9:1 molar ratio) and were multilamellar with sizes ranging from 250-370 nm. In vitro studies with human retinal pigment epithelial cell line (ARPE-19) showed that the b-LNAs were internalized by endocytosis and localized in endosomes. Exposure to UV light at $400 \mathrm{~mW} / \mathrm{cm}^{2}$ for $300+\mathrm{s}$ led to calcien release from b-LNAs, but not from liposomes that did not contain embedded nanoparticles.

\subsection{Drug delivery and hyperthermia}

Pradhan et al. [20] recently prepared folate (Fol) ligated magnetoliposomes (e-LNAs) via thin film hydration that contained co-encapsulated $60 \mathrm{~nm}$ iron oxide NPs and doxorubicin (DOX) in PBS. A proposed schematic of the e-LNA and the therapeutic concept is shown in Figure 2-2. The lipid components included DPPC, Chol, $\mathrm{PEG}_{2000-}$ DSPE, and Fol-PEG $2000-\mathrm{DSPE}$ and the liposomes exhibited a melting temperature near 
$41{ }^{\circ} \mathrm{C}$. The liposomes were $361 \mathrm{~nm}$ in diameter and polydispersed $(0.289)$, with a nanoparticle encapsulation efficiency of $24 \%$ (low, presumably attributed to the use of the thin film hydration method). The liposomes exhibited temperature-dependent DOX release (>2-fold increase from $37{ }^{\circ} \mathrm{C}$ to $43{ }^{\circ} \mathrm{C}$ ). Folate-receptor targeted uptake was demonstrated in HeLa and KB cell cultures, and reductions in cell viability were attributed to a synergistic effect of DOX and hyperthermia treatment achieved in the presence of an AC electromagnetic field. While not mentioned specifically, we speculate that liposomal heating by the nanoparticles may have aided DOX release.

Babincova et al. [96] prepared DPPC/PEG2000-DSPE LNAs via REV that contained both dextran-coated $\mathrm{Fe}_{3} \mathrm{O}_{4}$ and $\mathrm{C}_{60}$ fullerene (referred to as magnetofullerenosomes) to achieve magnetic targeting and photodynamic therapy (PDT), respectively. Bis(di-isobutyloctadecylsiloxy)-2,3-naphthalocyanato silicon (isoBO-SiNc), a photosensitizing agent, was also encapsulated. LNA performance for treating B16 pigmented melanoma was examined in vivo using a female mouse model. Without magnetic targeting, $\mathrm{C}_{60}$-mediated PDT followed by isoBO-SiNc-mediated PDT led to a significant reduction in tumor growth over 19 days relative to no treatment or isoBO-SiNc-mediated PDT alone. When magnetic targeting was applied $(0.32 \mathrm{~T}$ magnet placed on the tumor surface for $24 \mathrm{~h})$ there was negligible tumor growth over the same duration. 


\section{Expert Opinion}

Liposomes or NPs for therapeutic application are well established and have been approved by the FDA for clinical use. The combination of these two systems in hybrid structures represents a unique opportunity for achieving multiple therapeutic objectives. The liposomes can act to concentrate small NPs and shield them from the immune system. In turn, the NPs can be used to initiate and control drug release when exposed to external stimuli. However, the design and use of LNAs is still in its infancy. This is apparent from the literature where, for example, NP loading is expressed in multiple ways (e.g. total concentration; lipid:NP molar or mass ratios; liposome:NP surface area ratio). Further studies in the design and use of LNAs are needed addressing the following questions:

- How do physical and chemical interactions between NPs and the lipid bilayer affect LNA formation, structure, and stability? Encapsulated, decorated, and embedded NPs can affect lipid ordering (ordering or disordering are possible) and alter bilayer phase behavior. Ordering or phase state affects the permeability and stability of LNAs. These effects will be dependent upon the physical and chemical properties of the liposome, the NP, and the dispersing phase. Studies are needed that demonstrate the relationship between NP loading; liposome morphology, structure, and stability; and bilayer permeability for different LNA designs.

- Is the concept of local or nanoscale heating valid and under what conditions? LNAs designed for controlled release usually report a local or nanoscale heating mechanism where the NPs transfer heat to the LNA and causes a phase changes 
within the bilayer that trigger bilayer permeabilization. From this rationale, NPs in close proximity to bilayer, such as the case for embedment or decoration, would have the greatest effect on controlled release. However, other release mechanisms as a result of NP stimulation, working in tandem with bilayer heating, may be utilized (Figure 2-5). This statement is based in part on work by Keblinski et al. [65], who have shown that the theoretical temperature difference between a nanoparticle surface and the bulk phase due to EMF heating (light or RF) is almost negligible. This was further verified experimentally by Gupta et al. [97] and Bothun and Preiss [98] for $\mathrm{Fe}_{3} \mathrm{O}_{4}$ nanoparticles heated by RF. The fact that little difference between nanoscale and bulk heating is observed is due to the negligible heat transfer resistance in nanoscale films and rapid heat dissipation from the NP surface. Hence, NP heating does indeed occur and can trigger LNA release, but the heating is not localized to the LNA and no measurable difference can be observed with the bulk phase. It is unlikely that a lipid bilayer, even in contact with the nanoparticle surface, would exhibit a different temperature than that of the surrounding aqueous phase. 

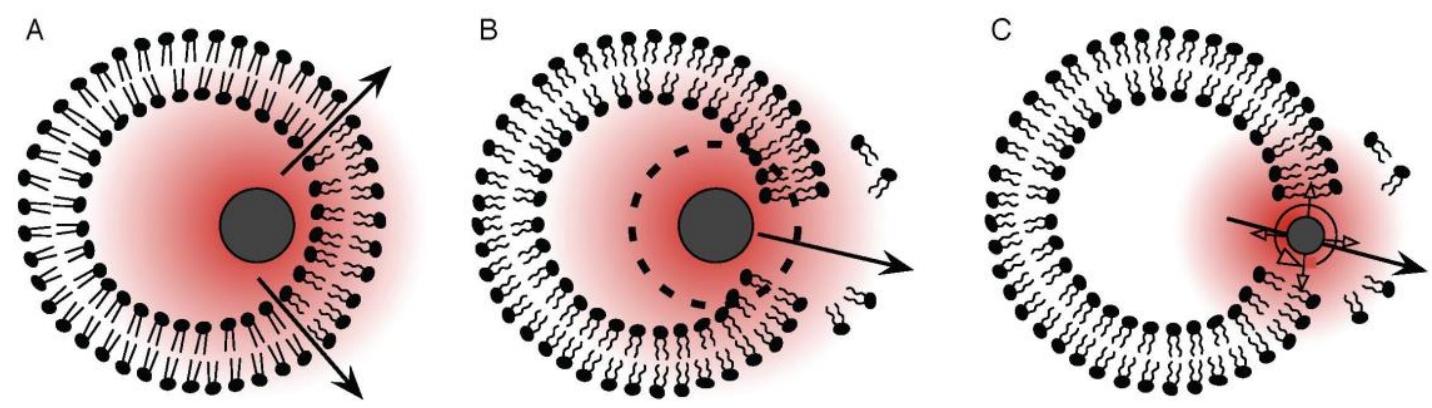

If gel phase lipids $\left(<T_{\mathrm{m}}\right)$
\{? fluid phase lipids $\left(>T_{\mathrm{m}}\right)$
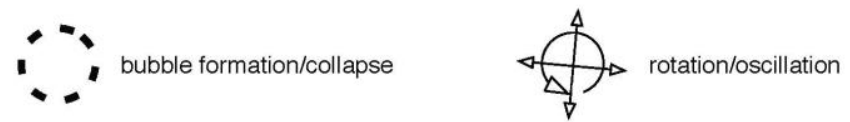

Figure 2-5. Nanoparticle heating controlled release mechanisms from liposomenanoparticle assemblies.

Controlled release mechanisms of LNAs. (A) Thermally-induced phase transitions due to NP heating where release is achieved through high diffusivity at the interface between gel and fluid lipid phases. (B) Mechanically-induced release where the bilayer is 'broken' due to bubble formation/rupture as a result of NP heating. (C) Mechanically-induced release where the bilayer is 'broken' due to NP rotation or oscillation at or within a LNA bilayer.

- What is LNA toxicity and how does the design strategy affect? It is unclear if LNA toxicity will stem from the liposomes or the NPs, or if a synergistic effect will occur. Toxicity, which is important from a clinical perspective, will depend on the LNA design and associated colloidal stability. For example, toxicity could be 'low' if an LNA can retain its NP agent until it reaches a target site. This could be the case for bound NPs formed by embedment or encapsulation. However, 'higher' toxicity could occur if the NPs are released during circulation (i.e. toxicity of liposomes + NPs). Release of encapsulated NPs could be attributed to liposome fusion or bilayer disintegration, release of embedded NPs may occur due to bilayer solubilization by surface-active agents, and release of decorated NPs may occur due to charge screening or competitive binding. 
- What clinical challenges exist to LNA-based therapeutic assemblies? As with toxicity, it is unclear if LNAs present unique clinical challenges beyond those reported for liposomes or nanoparticles [3, 4, 22]. These challenges include achieving biocompatibility, bioavailability, and cellular targeting and uptake. LNA structure, function, and stability will clearly impact how these challenges are addressed.

\section{Declaration of interest}

This work was supported by grants from the National Science Foundation (CBET0931875) and the NASA Rhode Island Space Grant Consortium.

\section{References}

Papers of special note have been highlighted as either of interest $\left(^{*}\right)$ or of considerable interest $(* *)$ to readers.

1. Koo OM, Rubinstein I, Onyuksel H. Role of nanotechnology in targeted drug delivery and imaging: a concise review. Nanomedicine 2005 Sep;1(3):193-212. 2. Brayden DJ. Controlled release technologies for drug delivery. Drug Discov Today 2003 Nov 1;8(21):976-8.

3. Petros RA, DeSimone JM. Strategies in the design of nanoparticles for therapeutic applications. Nat Rev Drug Discov 2010 Aug;9(8):615-27.

**A very nice recent review of nanoparticle design for therapeutic applications. 4. Phillips MA, Gran ML, Peppas NA. Targeted nanodelivery of drugs and diagnostics. Nano Today 2010 Apr;5(2):143-59. 
5. National Cancer Institute, 2005. [cited; Available from: www.nano.cancer.gov]

6. Babincova M, Cicmanec P, Altanerova V, Altaner C, Babinec P. AC-magnetic field controlled drug release from magnetoliposomes: design of a method for sitespecific chemotherapy. Bioelectrochemistry 2002 Jan;55(1-2):17-19.

**To our knowledge this is the first report on magnetoliposomes.

7. De Cuyper M, Joniau M. Magnetoliposomes: Formation and structural characterization. Eur Biophys J 1988;15:311-19.

8. Kullberg M, Mann K, Owens JL. Improved drug delivery to cancer cells: a method using magnetoliposomes that target epidermal growth factor receptors. Med Hypotheses 2005;64(3):468-70.

9. Shinkai M, Yanase M, Honda H, Wakabayashi T, Yoshida J, Kobayashi T. Intracellular hyperthermia for cancer using magnetite cationic liposomes: In vitro study. Jpn J Cancer Res 1996;87:1179-83.

10. Soenen SJH, Hodenius M, De Cuyper M. Magnetoliposomes: versatile innovative nanocolloids for use in biotechnology and biomedicine. Nanomed 2009 Feb;4(2):177-91.

*A thorough review of magnetoliposomes.

11. Viroonchatapan E, Sato H, Ueno M, Adachi I, Tazawa K, Horikoshi I. Release of 5-fluorouracil from thermosensitive magnetoliposomes induced by an electromagnetic field. J Control Release 1997 Jun 2;46(3):263-71.

12. Al-Jamal WT, Kostarelos K. Liposome-nanoparticle hybrids for multimodal diagnostic and therapeutic applications. Nanomed 2007 Feb;2(1):85-98. 
**An excellent review of liposome-nanoparticle hybrids (or LNAs as referred to herein).

13. Volodkin DV, Skirtach AG, Mohwald H. Near-IR Remote Release from Assemblies of Liposomes and Nanoparticles. Angew Chem Int Ed 2009;48(10):180709.

14. Drbohlavova J, Adam V, Kizek R, Hubalek J. Quantum Dots Characterization, Preparation and Usage in Biological Systems. Int J Mol Sci 2009 Feb;10(2):656-73.

15. Fukumori Y, Ichikawa H. Nanoparticles for cancer therapy and diagnosis. Advanced Powder Technology 2006;17(1):1-28.

16. Laurent S, Forge D, Port M, Roch A, Robic C, Elst LV, et al. Magnetic iron oxide nanoparticles: Synthesis, stabilization, vectorization, physicochemical characterizations, and biological applications. Chem Rev 2008 Jun;108(6):2064-110. 17. Rotomskis R, Streckyte G, Karabanovas V. Nanoparticles in diagnostics and therapy: towards nanomedicine. Medicina 2006;42(7):542-58.

18. Mornet S, Vasseur S, Grasset F, Duguet E. Magnetic nanoparticle design for medical diagnosis and therapy. J Mater Chem 2004;14:2161-75.

19. Li JL, Gu M. Gold-Nanoparticle-Enhanced Cancer Photothermal Therapy. Ieee J Sel Top Quant 2010 Jul-Aug;16(4):989-96.

20. Pradhan P, Giri J, Rieken F, Koch C, Mykhaylyk O, Doblinger M, et al. Targeted temperature sensitive magnetic liposomes for thermo-chemotherapy. $\mathbf{J}$ Control Release 2010 Feb 25;142(1):108-21. 
*Demonstrates potential synergistic effect of combined hyperthermia and drug release.

21. Zhang SL, Li J, Lykotrafitis G, Bao G, Suresh S. Size-Dependent Endocytosis of Nanoparticles. Adv Mater 2009 Jan 26;21(4):419-+.

22. Immordino ML, Dosio F, Cattel L. Stealth liposomes: review of the basic science, rationale, and clinical applications, existing and potential. Int J Nanomed 2006;1(3):297-315.

23. Chen YJ, Bose A, Bothun GD. Controlled Release from Bilayer-Decorated Magnetoliposomes via Electromagnetic Heating. ACS Nano 2010 Jun;4(6):3215-21.

24. Paasonen L, Laaksonen T, Johans C, Yliperttula M, Kontturi K, Urth A. Gold nanoparticles enable selective light-induced contents release from liposomes. J Control Release 2007 Sep 11;122(1):86-93.

*Comparitive study of photothermal release with different LNA designs.

25. Yu Y, Anthony SM, Zhang L, Bae SC, Granick S. Cationic nanoparticles stabilize zwitterionic liposomes better than anionic ones. J Phys Chem C 2007;111:8233-36.

26. Zhang LF, Granick S. How to stabilize phospholipid liposomes (using nanoparticles). Nano Lett 2006 Apr;6(4):694-98.

*Demonstrates how nanoparticle decoration can stabilize liposome dispersions. 27. Babincova M, Sourivong P, Leszczynska D, Babinec P. Fullerenosomes: Design of a novel nanomaterial for laser controlled topical drug release. Physica Medica 2003 Jul-Sep;19(3):213-16. 
28. Chen YJ, Bothun GD. Lipid-Assisted Formation and Dispersion of Aqueous and Bilayer-Embedded Nano-C60. Langmuir 2009 May 5;25(9):4875-79.

29. Doi Y, Ikeda A, Akiyama M, Nagano M, Shigematsu T, Ogawa T, et al. Intracellular uptake and photodynamic activity of water-soluble [60]- and [70]fullerenes incorporated in liposomes. Chem--Eur J 2008;14(Copyright (C) 2010 American Chemical Society (ACS). All Rights Reserved.):8892-97.

30. Hwang KC, Mauzerall D. Photoinduced Electron-Transport across a Lipid Bilayer Mediated by C70. Nature 1993 Jan 14;361(6408):138-40.

31. Ikeda A, Kikuchi J-I, inventors; National University Corporation Nara Institute of Science and Technology, Japan . assignee. Fullerene C70-containing liposome, method for producing the same, and use of the same. 2008.

32. Ikeda A, Nagano M, Akiyama M, Matsumoto M, Ito S, Mukai M, et al. Photodynamic activity of C70 caged within surface-cross-linked liposomes. Chem-Asian J 2009;4(Copyright (C) 2010 American Chemical Society (ACS). All Rights Reserved.):199-205.

33. Ikeda A, Sato T, Kitamura K, Nishiguchi K, Sasaki Y, Kikuchi J, et al. Efficient photocleavage of DNA utilising water-soluble lipid membrane-incorporated [60]fullerenes prepared using a [60]fullerene exchange method. Org Biomol Chem 2005 Aug 21;3(16):2907-09.

34. Jeng U-S, Hsu C-H, Lin T-L, Wu C-M, Chen H-L, Tai L-A, et al. Dispersion of fullerenes in phospholipid bilayers and the subsequent changes in the host bilayers. Physica B 2005;357:193-98. 
35. Niu SF, Manzerall D. Fast and efficient charge transport across a lipid bilayer is electronically mediated by C-70 fullerene aggregates. Journal Of The American Chemical Society 1996 Jun 19;118(24):5791-95.

36. Babincova M, Sourivong P, Leszczynska D, Babinec P. Photodynamic therapy of pigmented melanoma B16 using sterically stabilized fullerenosomes. Laser Phys Lett 2004 Sep;1(9):476-78.

37. Savarala S, Ahmed S, Ilies MA, Wunder SL. Formation and Colloidal Stability of DMPC Supported Lipid Bilayers on SiO2 Nanobeads. Langmuir $2010 \mathrm{Jul}$ 20;26(14):12081-88.

38. Troutier AL, Ladaviere C. An overview of lipid membrane supported by colloidal particles. Adv Colloid Interfac 2007 May 31;133(1):1-21.

39. Zhang LX, Sun XP, Song YH, Jiang X, Dong SJ, Wang EA.

Didodecyldimethylammonium bromide lipid bilayer-protected gold nanoparticles: Synthesis, characterization, and self-assembly. Langmuir 2006 Mar 14;22(6):2838-43. 40. Kshirsagar NA, Pandya SK, Kirodian GB, Sanath S. Liposomal drug delivery system from laboratory to clinic. J Postgrad Med 2005;51 Suppl 1:S5-15.

41. Samad A, Sultana Y, Aqil M. Liposomal drug delivery systems: an update review. Curr Drug Deliv 2007 Oct;4(4):297-305.

42. Torchilin VP. Recent advances with liposomes as pharmaceutical carriers. Nat Rev Drug Discov 2005 Feb;4(2):145-60. 
43. Mulder WJM, Strijkers GJ, van Tilborg GAF, Griffioen AW, Nicolay K. Lipid-based nanoparticles for contrast-enhanced MRI and molecular imaging. Nmr Biomed 2006 Feb;19(1):142-64.

44. Emerich DF, Thanos CG. The pinpoint promise of nanoparticle-based drug delivery and molecular diagnosis. Biomol Eng 2006;23:171-84.

45. Groneberg DA, Giersig M, Welte T, Pison U. Nanoparticle-based diagnosis and therapy. Current Drug Targets 2006 Jun;7(6):643-48.

46. Jin S, Ye K. Nanoparticle-mediated drug delivery and gene therapy. Biotechnol Prog 2007;23:32-41.

47. Michalet X, Pinaud FF, Bentolila LA, Tsay JM, Doose S, Li JJ, et al. Quantum dots for live cells, in vivo imaging, and diagnostics. Science 2005 Jan 28;307(5709):538-44.

48. Babincova M, Altanerova V, Altaner C, Bergemann C, Babinec P. In vitro analysis of cisplatin functionalized magnetic nanoparticles in combined cancer chemotherapy and electromagnetic hyperthermia. IEE T Nanobiosci 2008 Mar;7(1):15-19.

49. Bangham AD, Standish MM, Watkins JC. Diffusion of univalent ions across the lamellae of swollen phospholipids. J Mol Biol 1965;13:238-52.

50. Bangham AD, Horne RW. Negative staining of phospholipids and their structural modification by surface active agents as observed in the electron microscope. J Mol Biol 1964;8:660-68. 
51. Papahadjopoulos D, Ohki S. Stability of asymmetric phospholipid membranes. Science 1969 May 30;164(883):1075-7.

52. Gregoriadis G. Drug entrapment in liposomes. FEBS Lett 1973 Nov $1 ; 36(3): 292-6$.

53. Pagano RE, Weinstein JN. Interactions of Liposomes with Mammalian-Cells. Annu Rev Biophys Bio 1978;7:435-68.

54. Huang X, El-Sayed IH, El-Sayed MA. Applications of gold nanorods for cancer imaging and photothermal therapy. Methods Mol Biol 2010;624:343-57.

55. Oldenburg SJ, Jackson JB, Westcott SL, Halas NJ. Infrared extinction properties of gold nanoshells. Appl Phys Lett 1999 Nov 8;75(19):2897-99.

56. Krpetic Z, Nativo P, See V, Prior IA, Brust M, Volk M. Inflicting Controlled Nonthermal Damage to Subcellular Structures by Laser-Activated Gold Nanoparticles. Nano Letters 2010 Nov;10(11):4549-54.

57. Gannon CJ, Patra CR, Bhattacharya R, Mukherjee P, Curley SA. Intracellular gold nanoparticles enhance non-invasive radiofrequency thermal destruction of human gastrointestinal cancer cells. J Nanobiotechnology 2008;6:2.

58. Pankhurst QA, Connolly J, Jones SK, Dobson J. Applications of magnetic nanoparticles in biomedicine. J Phys D 2003;36:167-81.

59. Brezovich IA. Low frequency hyperthermia. Med Phys Monograph $1988 ; 16: 82-111$ 
60. Hildebrandt B, Wust P, Ahlers O, Dieing A, Sreenivasa G, Kerner T, et al. The cellular and molecular basis of hyperthermia. Crit Rev Oncol Hematol 2002 Jul;43(1):33-56.

61. Hsu MH, Su YC. Iron-oxide embedded solid lipid nanoparticles for magnetically controlled heating and drug delivery. Biomed Microdevices 2008 Dec;10(6):785-93.

62. Ito A, Kuga Y, Honda H, Kikkawa H, Horiuchi A, Watanabe Y, et al. Magnetite nanoparticle-loaded anti-HER2 immunoliposomes for combination of antibody therapy with hyperthermia. Cancer Lett 2004 Aug 30;212(2):167-75.

63. Pennes HH. Analysis of tissue and arterial temperature in the resting human forearm. J Appl Physiol 1948;1:93-122.

64. Rast L, Harrison JG. Computational modeling of electromagnetically induced heating of magnetic manoparticle materials for hyperthermic cancer treatment. PIERS Online 2010;6(7):690-94.

65. Keblinski P, Cahill DG, Bodapati A, Sullivan CR, Taton TA. Limits of localized heating by electromagnetically excited nanoparticles. J Appl Phys 2006 Sep $1 ; 100(5):-$.

*One of the first studies challenging the concept of nanoscale heating by nanoparticles using electromagnetic fields.

66. Xu RZ, Zhang Y, Ma M, Xia JG, Liu JW, Guo QZ, et al. Measurement of specific absorption rate and thermal simulation for arterial embolization hyperthermia in the Maghemite-Gelled model. Ieee T Magn 2007 Mar;43(3):1078-85. 
67. Hong K, Friend DS, Glabe CG, Papahadjopoulos D. Liposomes containing colloidal gold are a useful probe of liposome-cell interactions. Biochim Biophys Acta 1983 Jul 13;732(1):320-3.

68. Zheng S, Zheng Y, Beissinger RL, Fresco R. Microencapsulation of Hemoglobin in Liposomes Using a Double Emulsion, Film Dehydration Rehydration Approach. Biochimica Et Biophysica Acta-Biomembranes 1994 Dec 30;1196(2):12330.

69. Szoka F, Jr., Papahadjopoulos D. Procedure for preparation of liposomes with large internal aqueous space and high capture by reverse-phase evaporation. Proc Natl Acad Sci USA 1978 Sep;75(9):4194-8.

70. Wijaya A, Hamad-Schifferli K. High-density encapsulation of $\mathrm{Fe}_{3} \mathrm{O}_{4}$ nanoparticles in lipid vesicles. Langmuir 2007;23(19):9546-50.

*Demonstrates high-density encapsulation in magnetoliposomes.

71. Lipowsky R, Dobereiner HG. Vesicles in contact with nanoparticles and colloids. Europhys Lett 1998 Jul 15;43(2):219-25.

72. Pradhan P, Giri J, Banerjee R, Bellage J, Bahadur D. Preparation and characterization of manganese ferrite-based magnetic liposomes for hyperthermia treatment of cancer. J Magn Magn Mater 2007;311:208-15.

73. Sabate R, Barnadas-Rodriguez R, Callejas-Fernandez J, Hidalgo-Alvarez R, Estelrich J. Preparation and characterization of extruded magnetoliposomes. International Journal of Pharmaceutics 2008 Jan 22;347(1-2):156-62. 
74. Gomes JFPS, Rank A, Kronenberger A, Fritz J, Winterhalter M, Ramaye Y. Polyelectrolyte-coated unilamellar nanometer-sized magnetic liposomes. Langmuir 2009 Jun 16;25(12):6793-9.

75. Jang H, Pell LE, Korgel BA, English DS. Photoluminescence quenching of silicon nanoparticles in phospholipid vesicle bilayers. J Photochem Photobiol A 2003;158:111-17.

*Describes the critical nanoparticle size for bilayer embedment (see also references 69, 76, and 77).

76. Bothun GD. Hydrophobic silver nanoparticles trapped in lipid bilayers: Size distribution, bilayer phase behavior, and optical properties. J Nanobiotechnology $2008 ; 6: 13$

77. Al-Jamal WT, Al-Jamal KT, Tian B, Lacerda L, Bomans PH, Frederik PM, et al. Lipid-quantum dot bilayer vesicles enhance tumor cell uptake and retention in vitro and in vivo. ACS Nano 2008;2(3):408-18.

78. Bothun GD, Rabideau AE, Stoner MA. Hepatoma Cell Uptake of Cationic Multifluorescent Quantum Dot Liposomes. J Phys Chem B 2009 Jun 4;113(22):772528.

79. Park S-H, Oh S-G, Mun J-Y, Han S-S. Effects of silver nanoparticles on the fluidity of bilayer in phospholipid liposome. Coll Surf B 2005;44:117-22.

80. Park S-H, Oh S-G, Mun J-Y, Han S-S. Loading of gold nanoparticles inside the DPPC bilayers of liposome and their effects on membrane fluidities. Coll Surf B 2006;48:112-18. 
81. Binder WH, Sachsenhofer R, Farnik D, Blaas D. Guiding the location of nanoparticles into vesicular structures: a morphological study. Phys Chem Chem Phys 2007;9(48):6435-41.

82. Rasch MR, Rossinyol E, Hueso JL, Goodfellow BW, Arbiol J, Korgel BA. Hydrophobic Gold Nanoparticle Self-Assembly with Phosphatidylcholine Lipid: Membrane-Loaded and Janus Vesicles. Nano Lett 2010 Sep;10(9):3733-39. **Depicts how embedded Au nanoparticles cluster in LNAs and how LNA preparation yields different structures.

83. Ginzburg VV, Balijepalli S. Modeling the thermodynamics of the interaction of nanoparticles with cell membranes. Nano Lett 2007;7(12):3716-22.

84. Wi HS, Lee K, Pak HK. Interfacial energy consideration in the organization of a quantum dot-lipid mixed system. J Phys Cond Mat 2008 Dec 10;20(49):1-6.

85. Al-Jamal WT, Al-Jamal KT, Bomans PH, Frederik PM, Kostarelos K. Functionalized-quantum-dot-liposome hybrids as multimodal nanoparticles for cancer. Small 2008 Sep;4(9):1406-15.

86. Wang B, Zhang LF, Bae SC, Granick S. Nanoparticle-induced surface reconstruction of phospholipid membranes. Proc Nat Acad Sci USA 2008 Nov 25;105(47):18171-75.

*A key study that demonstrates how nanoparticle binding to liposomes can induce phase separation. 
87. Sau TP, Urban AS, Dondapati SK, Fedoruk M, Horton MR, Rogach AL, et al. Controlling loading and optical properties of gold nanoparticles on liposome membranes. Coll Surf A 2009;342:92-96.

*Compares Au nanoparticle binding as a function of liposome surface charge.

88. Kojima C, Hirano Y, Yuba E, Harada A, Kono K. Preparation and characterization of complexes of liposomes with gold nanoparticles. Coll Surf B 2008 Oct 15;66(2):246-52.

89. Pornpattananangkul D, Olson S, Aryal S, Sartor M, Huang CM, Vecchio K, et al. Stimuli-Responsive Liposome Fusion Mediated by Gold Nanoparticles. Acs Nano 2010 Apr;4(4):1935-42.

90. Mart RJ, Liem KP, Webb SJ. Creating Functional Vesicle Assemblies from Vesicles and Nanoparticles. Pharmaceutical Research 2009 Jul;26(7):1701-10.

91. Anderson LJE, Hansen E, Lukianova-Hleb EY, Hafner JH, Lapotko DO. Optically guided controlled release from liposomes with tunable plasmonic nanobubbles. Journal of Controlled Release 2010 Jun 1;144(2):151-58.

92. Wu GH, Milkhailovsky A, Khant HA, Fu C, Chiu W, Zasadzinski JA. Remotely triggered liposome release by near-infrared light absorption via hollow gold nanoshells. J Am Chem Soc 2008 Jul 2;130(26):8175-77.

**Demonstrates ability to use encapsulated or decorated hollow Au nanoshells for laser-triggered release. 
93. Tai LA, Tsai PJ, Wang YC, Wang YJ, Lo LW, Yang CS. Thermosensitive liposomes entrapping iron oxide nanoparticles for controllable drug release. Nanotechnology 2009 Apr 1;20(13):-.

94. Chithrani DB, Dunne M, Stewart J, Allen C, Jaffray DA. Cellular uptake and transport of gold nanoparticles incorporated in a liposomal carrier'. NanomedNanotechnol 2010 Feb;6(1):161-69.

95. Paasonen L, Sipila T, Subrizi A, Laurinmaki P, Butcher SJ, Rappolt M, et al. Gold-embedded photosensitive liposomes for drug delivery: Triggering mechanism and intracellular release. J Control Release 2010 Oct 1;147(1):136-43.

96. Babinec P, Babincova M, Sourivong P, Leszczynska D. Efficient treatment of pigmented B16 melanoma using photosensitized long-circulating magnetofullerenosomes. J Magn Magn Mater 2005 May 1;293(1):394-97.

97. Gupta A, Kane RS, Borca-Tasciuc DA. Local temperature measurement in the vicinity of electromagnetically heated magnetite and gold nanoparticles. J Appl Phys 2010 Sep 15;108(6):-.

98. Bothun GD, Preiss MR. Bilayer heating in magnetite nanoparticle-liposome dispersions via fluorescence anisotropy. J Coll Inter Sci 2011; in press.

99. Larsen BA, Haag MA, Serkova NJ, Shroyer KR, Stoldt CR. Controlled aggregation of superparamagnetic iron oxide nanoparticles for the development of molecular magnetic resonance imaging probes. Nanotechnology 2008 Jul 2;19(26):-. 
100. Zhu L, Huo ZL, Wang LL, Tong X, Xiao Y, Ni KY. Targeted delivery of methotrexate to skeletal muscular tissue by thermosensitive magnetoliposomes. International Journal of Pharmaceutics 2009 Mar 31;370(1-2):136-43. 


\title{
CHAPTER 3
}

\section{Local Heating in Magnetite Nanoparticle-Liposome Dispersions via Fluorescence}

\author{
Anisotropy \\ Geoffrey D. Bothun ${ }^{1 \dagger}$ and Matthew R. Preiss ${ }^{1}$ \\ Published in: Journal of Colloid and Interface Science 357(1) 70-74 (2011). \\ ${ }^{1}$ University of Rhode Island, Department of Chemical Engineering, \\ Kingston, RI, USA
}

${ }^{\dagger}$ Author for correspondence: Geoffrey D Bothun

Department of Chemical Engineering

University of Rhode Island

205 Crawford Hall, 16 Greenhouse Road,

Kingston, RI, 02881, USA

Phone: +1-401-874-9518

Email: gbothun@uri.edu 


\section{Abstract}

Temperature measurements have been made within magnetite $\left(\mathrm{Fe}_{3} \mathrm{O}_{4}\right)$ nanoparticle-liposome dispersions subjected to electromagnetic field at radiofrequency (RF) heating based on the fluorescence anisotropy of diphenylhexatriene (DPH) embedded within the bilayer. Incorporating cholesterol within dipalmitoylphosphatidylcholine (DPPC) bilayers broadened the anisotropy window associated with lipid melting. Cryogenic transmission electron microscopy showed that the dispersions contained magnetoliposomes with nanoparticle aggregates at both low and high encapsulation densities. RF heating results demonstrated the ability to measure the temperature of the ML bilayer with on/off RF cycles using DPH anisotropy. These measurements reflected the temperature of the bulk aqueous phase, which is consistent with previous work showing rapid heat dissipation from a nanoparticle surface during RF heating and a negligible difference between surface and bulk temperature.

Keywords: Magnetoliposome; Magnetic nanoparticle; Electromagnetic heating; Hyperthermia; Lipid bilayer 


\section{Introduction}

Magnetoliposomes (MLs) consist of iron oxide nanoparticles encapsulated within lipid bilayer vesicles or liposomes [1-4]. Two common magnetoliposome structures formed by encapsulating hydrophilic magnetic nanoparticles are shown in Figure 3-1. The lipid bilayer coating provides a biocompatible means of dispersing the nanoparticles in aqueous milieu. In addition, the liposome structure provides a means

of delivering hydrophobic (within the bilayer) or hydrophilic (within the aqueous core) molecules co-encapsulated with the nanoparticles. ML delivery to target sites in vivo can be achieved physically, by guiding the MLs using magnets placed on a body's exterior [5], as well as chemically using cationic lipids or ligand-conjugated lipids with selectivity for specific cellular receptors $[5,6]$. Pradhan et al, for example, recently showed that the inclusion of a folate-conjugated lipid can be used to target MLs to cancer cells via folate receptor (FR) binding and FR-mediated endocytosis [7].

A unique property of MLs is the ability to heat the encapsulated magnetic nanoparticles, typically iron oxide (maghemite, $\square-\mathrm{Fe}_{2} \mathrm{O}_{3}$ or magnetite, $\mathrm{Fe}_{3} \mathrm{O}_{4}$ ), using external alternating current electromagnetic fields (EMFs) operating at radiofrequencies (RFs) $[6,8]$. With this mode of heating, magnetic losses are converted to energy. The magnetic losses are attributed to Neel relaxation, which is due to rapidly alternating magnetic dipole moments, and Brownian relaxation, which is due to nanoparticle rotation (i.e. friction losses) [9]. ML heating using physiologically compatible or benign RF strengths can provide local hyperthermia treatment [10] or combined hyperthermia and drug delivery [7]. This is attributed to radiofrequencies easily penetrating the body and being non-invasive for up to 1 hour if 
the product $H f$, where $H$ is the field amplitude (current $\times$ number of coils per length) and $f$ is the frequency, is below $4.85 \times 10^{5} \mathrm{kA} \mathrm{m}^{-1} \mathrm{~s}^{-1}$ [11]. With respect to drug delivery, initiating and controlling drug release from MLs has been attributed to the ability to manipulate the phase behavior and diffusivity of the lipid bilayer [7, 12-14]. Combining the ability to guide MLs using magnets and trigger release with RFs can overcome a major challenge of 'conventional' liposomes for drug delivery - obtaining high liposome accumulation at a target site and achieving a desired controlled release profile [15].

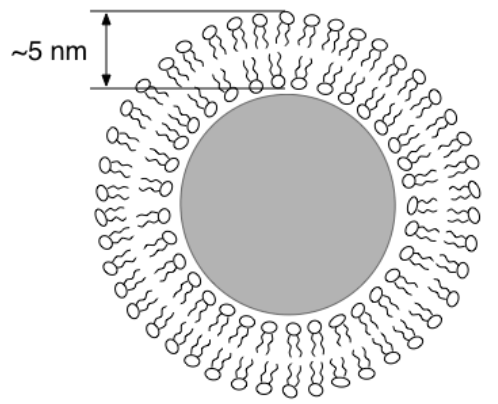
Single nanoparticle or nanoparticle aggregate
encapsulated by a supported lipid bilayer

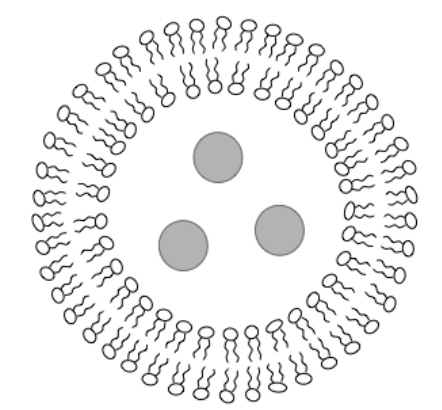

Multiple nanoparticles encapsulated within a liposome

\section{Figure 3-1. Magnetoliposome structures.}

Magnetoliposome structures consisting of (top) a single nanoparticle encapsulated within a supported lipid bilayer and (bottom) multiple nanoparticles encapsulated within a liposome. 
Challenges to ML-based hyperthermia and drug release include measuring changes in temperature without the use of invasive probes and at the site of interest i.e. local temperatures in the vicinity of the nanoparticles [8]. With respect to hyperthermia, the local temperature in vivo is important in cancer treatment where elevated temperatures must be maintained for a period of time to cause cell death (e.g. $42{ }^{\circ} \mathrm{C}$ or a $\Delta \mathrm{T}$ of $5{ }^{\circ} \mathrm{C}$ from physiological temperature for $>30 \mathrm{~min}$ ) [16]. In this case questions arise concerning what minimum cellular nanoparticle (or ML) loading is needed to achieve sufficient heating, as well as the ability to selectively heat the cancer cells or tumor mass and not damage healthy cells or tissues [9]. In turn, for drug release applications, the heat delivered to or generated within the MLs may be used to control transbilayer release by raising the bilayer to its melting temperature [1, 12, 17]. However, there is some controversy associated with this statement. Keblinski et al. [18] have shown that the theoretical temperature difference between a nanoparticle surface and the bulk phase due to EMF heating is almost negligible. This was further verified by Gupta et al. [19] for $\mathrm{Fe}_{3} \mathrm{O}_{4}$ nanoparticles heated by RFs. In this work quantum dots were mixed or covalently anchored to the nanoparticles as temperature probes to compare bulk verse local temperature.

In this work we describe an in situ spectrofluorometric-based approach to determine the effect of RF heating on the temperature of liposomal bilayers in a $\mathrm{Fe}_{3} \mathrm{O}_{4}$ nanoparticle-liposome dispersion containing MLs. The approach is based on the anisotropy, $\langle r\rangle$, of a lipid bilayer probe molecule, diphenylhexatriene (DPH), and the proportionality among anisotropy, bilayer viscosity, and lipid phase state, which depend on temperature [20]. Nanoparticle-liposome dispersions were prepared with 
dipalmitoylphosphatidylcholine (DPPC) and contained 25 mol\% cholesterol and 0.2 mol\% DPH. While cholesterol is known to stabilize liposomes, in this work it was added specifically to broaden the main phase transition or melting temperature region $[21,22]$. This provided a large heating window for anisotropy measurements.

Calculated (via $\langle r\rangle$ ) and measured temperatures were compared to differentiate between local and bulk heating relative to MLs as a function of time during on/off RF cycles.

\section{Materials and methods}

\subsection{Chemicals}

Dipalmitoylphosphatidylcholine (DPPC) and cholesterol were purchased from Avanti Polar Lipids, and diphenylhexatriene (DPH) from Sigma Chemical Company. The aqueous $\mathrm{Fe}_{3} \mathrm{O}_{4}$ nanoparticle solution was purchased from Ferrotec $\mathrm{GmbH}$ (EMG 705 ferrofluid, 3.9 vol\%). Previous work has shown that in this solution the particles are well dispersed with an average diameter of $12.5 \pm 3.4 \mathrm{~nm}$ [23]. Sterile deionized water was obtained from a Millipore Direct-3Q purification system. All materials were used as received with the exception of dilution.

\subsection{Preparation of $\mathrm{Fe}_{3} \mathrm{O}_{4}$ nanoparticle-liposome dispersions}

Samples were prepared by reverse phase evaporation [24] (Buchi Rotavapor R215, Zurich, Switzerland), similar to the procedure by Wijaya and Hamad-Schifferli [23]. The following components were added to a $25 \mathrm{ml}$ round bottom flask: $320 \mu \mathrm{l}$ of $10 \mathrm{mM}$ DPPC in chloroform, $80 \mu \mathrm{l}$ of $10 \mathrm{mM}$ cholesterol in chloroform, $40 \mu \mathrm{l}$ of 0.2 mM DPH in tetrahydrofuran, and $40 \mu \mathrm{l}$ of ferrofluid. To this, $4 \mathrm{ml}$ of DI water and 16 
$\mathrm{ml}$ of chloroform were added and an emulsion was formed by bath sonication for 5 min at $50{ }^{\circ} \mathrm{C}$, which is above the DPPC melting temperature $\left(T_{\mathrm{m}}\right)$ of $42{ }^{\circ} \mathrm{C}$ [25]. The sample was cooled at room temperature for $30 \mathrm{~min}$. The solvent phase was then removed by rotary evaporation at $25^{\circ} \mathrm{C}$ and $100 \mathrm{RPM}$ under $300 \mathrm{mbar}$ for $30 \mathrm{~min}$ followed by $200 \mathrm{mbar}$ for $2 \mathrm{~h}$. The final dispersions contained $0.8 \mathrm{mM}$ DPPC and 1 $\mathrm{mM} \mathrm{Fe}_{3} \mathrm{O}_{4}$, which was equivalent to approximately $0.23 \mathrm{mg} \mathrm{ml}^{-1}$, equivalent to $0.23 \%$ mass fraction in water and $287.5 \mathrm{~g} \mathrm{Fe}_{3} \mathrm{O}_{4} \mathrm{~mol}^{-1} \mathrm{DPPC}$.

Nanoparticle encapsulation efficiency was estimated using a centrifugation procedure employed by Pradhan et al. [10]. Briefly, 0.9 wt $\% \mathrm{NaCl}$ was added to the samples (in DI water) at a volume ratio of 1:1. This led to the aggregation of unencapsulated nanoparticles via charge screening. The solution was then centrifuged at $1000 \mathrm{~g}$ for $10 \mathrm{~min}$ at $4{ }^{\circ} \mathrm{C}$. The supernatant containing MLs and (if present) empty liposomes was removed and the precipitate was resuspended in DI water, digested in nitric acid, and analyzed by inductively coupled plasma mass spectrometry (ICP-MS, Thermo Electron X Series, Waltham, MA). Encapsulation was calculated based on the $57 \mathrm{Fe}$ isotope.

\subsection{Cryogenic transmission electron microscopy (cryo-TEM)}

The nanoparticle-liposome dispersions were prepared for cryo-TEM at $25{ }^{\circ} \mathrm{C}$ using a Vitrobot (FEI Company). A quantifoil grid with $2 \mu \mathrm{m}$ carbon holes on 200 square mesh copper (Electron Microscopy Sciences, Hatfield, PA) was immersed in the sample, blotted to reduce film thickness, and vitrified in liquid ethane. The sample was transferred to liquid nitrogen for storage and imaging, which was performed in a 
cooled stage (Model 915, Gatan Inc., Pleasonton, CA) at $200 \mathrm{kV}$ using a JEOL JEM2100F TEM (Peabody, MA). Size analysis was performed using ImageJ software [26]. The average size reported was based on all liposomes observed in the cryo-TEM micrographs.

\subsection{RF heating}

RF heating was performed using a $1 \mathrm{~kW}$ Hotshot $^{\mathrm{TM}}$ (Ameritherm Inc., Scottsville, NY) operating from 50 to $250 \mathrm{~A}$ and 291 to $287 \mathrm{kHz}$, respectively. The specific absorption rates $(S A R)$ of the nanoparticles were measured using a 3-turn copper coil with a $4.5 \mathrm{~cm}$ outer diameter. To determine $S A R$ values, sample temperatures were measured as a function of nanoparticle concentration and electromagnetic field strength, $H f$, which was varied between $1 \times 10^{5}$ and $5 \times 10^{5} \mathrm{kA} \mathrm{m}^{-1} \mathrm{~s}^{-1} . S A R\left(\mathrm{~W} \mathrm{~g}^{-1}\right)$ values were calculated from the initial slope of adiabatic temperature rise of the samples, $\Delta T / \Delta t=S A R m_{N P} /\left(\bar{c}_{p} m_{V}\right)$ (Eq. 1), where $m_{N P}$ and $m_{V}$ are the mass of the nanoparticles and sample volume, respectively, and $\bar{c}_{p}=\phi c_{p, N}+(1-\phi) c_{p, F}$ is the average heat capacity based on the nanoparticle volume fraction in the sample, $\phi$.

For in situ fluorescence anisotropy measurements, the 3-turn coil was placed around the cuvette holder within the sample compartment of the spectrophotometer. The coil was separated slightly to allow for the excitation and emission light paths. The sample was diluted 7-fold (0.11 mM DPPC, $\left.0.03 \mathrm{mg} \mathrm{Fe}_{3} \mathrm{O}_{4} \mathrm{ml}^{-1}\right)$, placed within a quartz cuvette (10 mm path length), and subjected to RF-heating cycles as a function of time at $5 \times 10^{5} \mathrm{kA} \mathrm{m}^{-1} \mathrm{~s}^{-1}(250 \mathrm{~A}$ and $281 \mathrm{kHz})$, which was the maximum field strength used for $S A R$. The bulk solution temperature was measured intermittently 
using a temperature probe. Steady-state DPH anisotropy within the DPPC bilayer was determined with at $\lambda_{\mathrm{ex}}=350 \mathrm{~nm}$ and $\lambda_{\mathrm{em}}=452 \mathrm{~nm}$ using the expression $\langle r\rangle=(I \mathrm{vv}-$ $I \mathrm{vH}) /(I \mathrm{vv}+G I \mathrm{vH})($ Eq. 2) where $I$ represents the fluorescence emission intensity, $\mathrm{V}$ and $\mathrm{H}$ represent the vertical and horizontal orientation of the excitation and emission polarizers, and $G=I_{\mathrm{HV}} / I_{\mathrm{HH}}$ accounts for the sensitivity of the instrument towards vertically and horizontally polarized light. The excitation and emission slit widths were set at $10 \mathrm{~nm}$.

\section{Results and discussion}

\subsection{Nanoparticle RF-heating}

$S A R$ values were determined as a function of RF field strength and nanoparticle mass fraction. The SAR increased with increasing field strength and decreased slightly with increasing nanoparticle mass fraction (Figure 3-2). Given that $S A R$ values are normalized for nanoparticle concentration, the decrease with increasing nanoparticle mass fraction may reflect greater heat loss from the sample tubes to the surroundings prior to temperature measurements. 


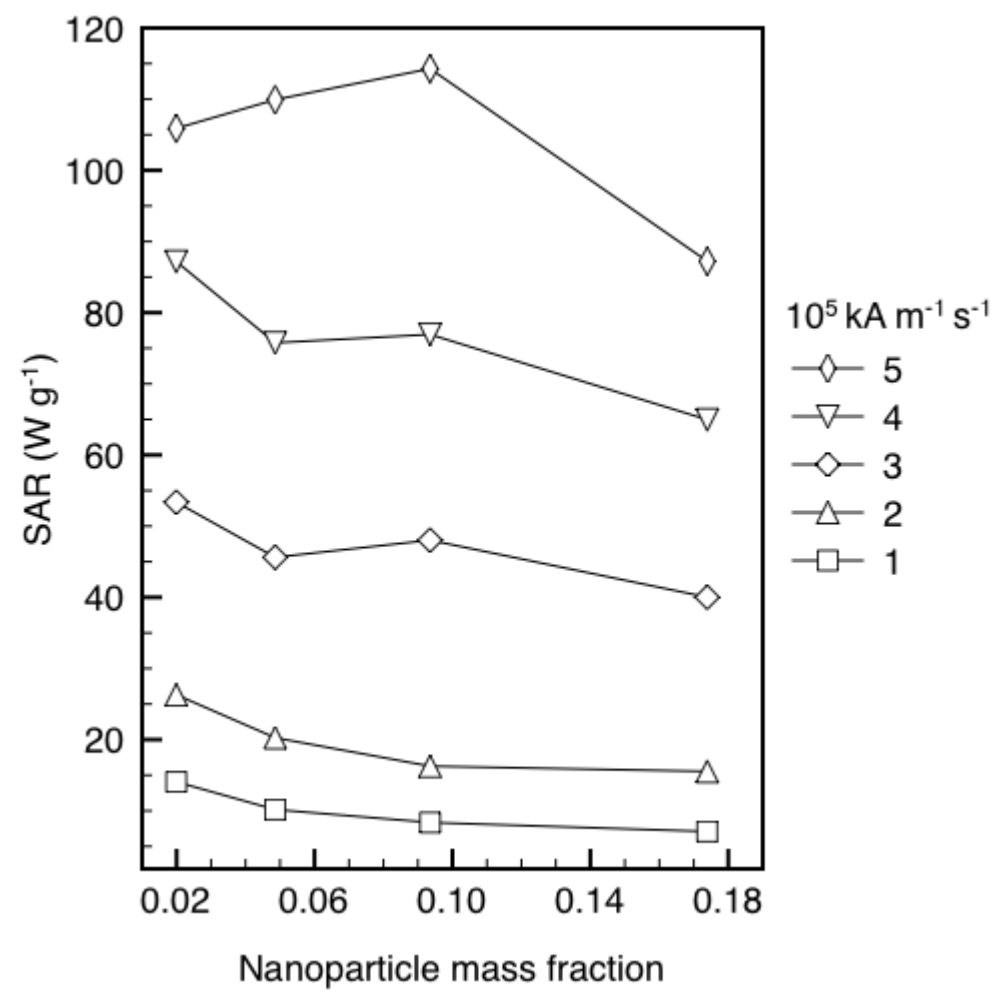

Figure 3-2. Specific absorption rate $(\mathrm{SAR})$ of the $\mathrm{Fe}_{3} \mathrm{O}_{4}$ nanoparticles as a function of RF field strength ( $H f$, in legend) and nanoparticle mass fraction (in water).

\subsection{Cryo-TEM and DLS analysis}

Cryo-TEM micrographs of the nanoparticle-liposome dispersion taken one week after preparation are shown in Figure 3-3. Unilamellar liposomes with and without smaller encapsulated unilamellar liposomes were observed. The liposomes had an average diameter of $317 \pm 192 \mathrm{~nm}$ and exhibited significant polydispersity (based on all structures observed). Polydispersity can be attributed to the fact that no post-formation processing was performed, and was not a concern as DPH anisotropy is weakly dependent on liposome size (excluding high curvature effects in small liposomes). Based on all the liposomal structures shown in Figure 3-3, 30\% contained 
encapsulated nanoparticles (Figure 3-3, arrowed region 2) or nanoparticle aggregates (Figure 3-3, arrowed region 1) indicative of MLs. MLs were also observed in ruptured or rupturing states with nanoparticle aggregates being released into the bulk aqueous phase (Figure 3-3, arrowed region 3).

It should be noted that the percentage of liposomes containing nanoparticles or nanoparticle aggregates is not equivalent to encapsulation efficiency. An encapsulation efficiency of $67 \%$ was measured one day after sample preparation based on ICP-MS analysis of precipitate and supernatant fractions after centrifugation [10]. Comparing cryo-TEM and encapsulation efficiency results suggests that most of the nanoparticles were encapsulated as aggregates within a small population of liposomes and/or that encapsulated nanoparticles were released due to ML rupture. Nanoparticle release from MLs has been observed by Wijaya and Hamad-Schifferli for high-density nanoparticle-loaded vesicles (HNLVs) prepared using DPPC and the same ferrofluid [23]. In their study the samples were analyzed $30 \mathrm{~min}$ after preparation and release was attributed to incomplete closure, which reduces encapsulation efficiency. In the present work, cryo-TEM was conducted one week after ML formation (stored at 25 ${ }^{\circ} \mathrm{C}$ ) and rupture was still clearly evident. 

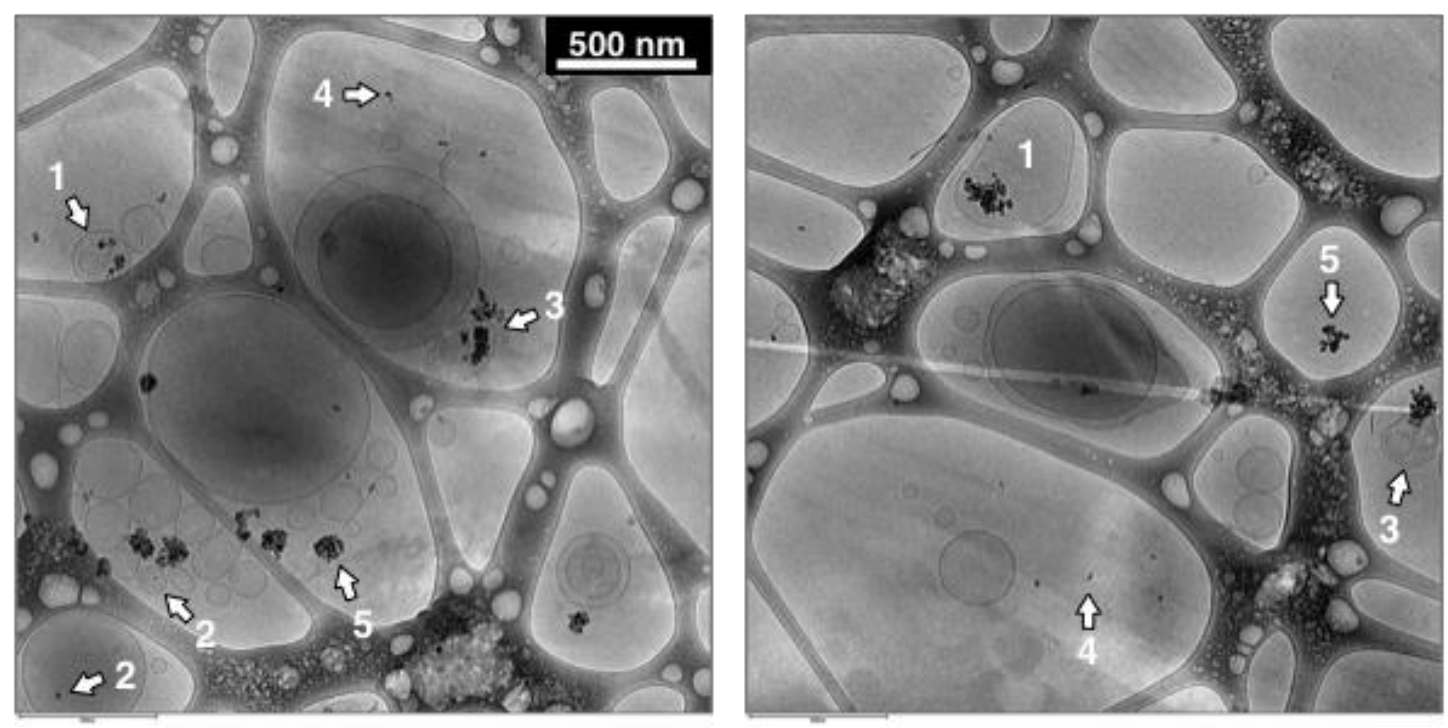

Figure 3-3. Cryo-TEM micrographs of the nanoparticle-liposome dispersion. The images depict (1) magnetoliposomes containing encapsulated nanoparticle aggregates or (2) single nanoparticles; (3) magnetoliposomes rupturing and nanoparticle release; and unencapsulated (4) single nanoparticles and (5) nanoparticle aggregates. The scale bar represents $500 \mathrm{~nm}$ and is common to both images.

\subsection{DPH anisotropy as a function of temperature and RF-heating}

DPH anisotropy for liposomes (no nanoparticles) and nanoparticle-liposome dispersions with MLs (no RF-heating) as a function of temperature is shown in Figure 3-4. The results indicate a $T_{\mathrm{m}}$ of $43^{\circ} \mathrm{C}$, where the bilayer transitions from an ordered gel to disordered fluid phase, and a broad melting region from 31 to $54{ }^{\circ} \mathrm{C}$. These results are consistent with the effects of cholesterol on DPPC phase behavior and demonstrate that the presence of nanoparticles did not influence lipid melting [21]. A least squares regression fit of the liposome-nanoparticle dispersion data provided a calibration curve $\left(T=-5865.0<r>^{3}+4204.9<r>^{2}-1122.8<r>+154.4 ; \mathrm{R}^{2}=0.97\right)$ (Eq.

3) for determining temperature as a function of DPH anisotropy during RF-heating. 


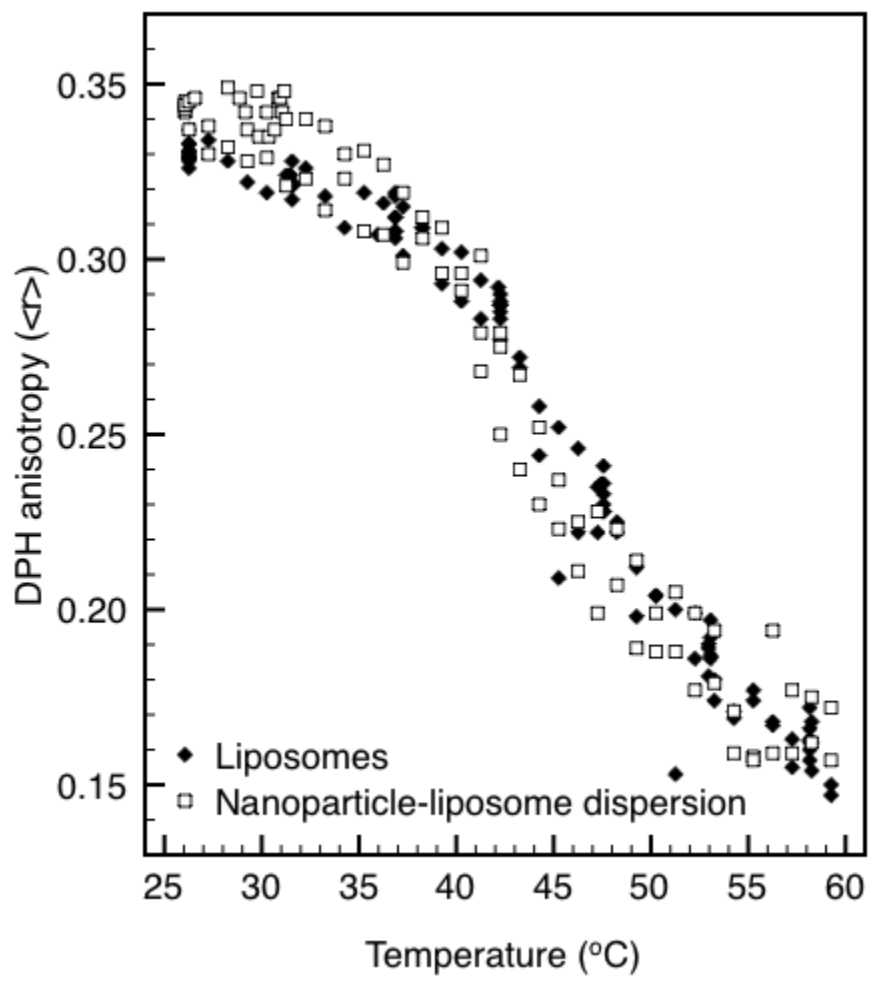

Figure 3-4. Melting transition determined by DPH anisotropy.

The reduction in DPH anisotropy with increasing temperature reflects an increase in bilayer fluidity, or decrease in microviscosity, as the bilayers transition from a gel to a fluid phase.

DPH anisotropy was measured in situ during RF-heating $\left(5 \times 10^{5} \mathrm{kA} \mathrm{m}^{-1} \mathrm{~s}^{-1}\right)$ as a function of time. Heating was initiated at time zero and cycled between off and on. The $T$ versus $\langle r\rangle$ calibration curve (Eq. 3 ) was used to calculate temperature. Prior to conducting the experiment, a cuvette containing water was heated by RF for $1 \mathrm{~h}$. The temperature rise from this test was from 25 to $26^{\circ} \mathrm{C}$, which demonstrates that heating of the cuvette, cuvette holder, and base within the spectrophotometer sample compartment was minimal and its contribution to sample heating was negligible. 
A rapid increase in the calculated temperature $(v i a\langle r\rangle)$ was observed with EMF heating up to $1400 \mathrm{~s}$ (Fig. 3-5). The temperature changes with on/off EMF cycling, between 1400 and $1450 \mathrm{~s}$ and 1450 and $2100 \mathrm{~s}$, demonstrate the response and reversibility of ML membrane fluidity and its use for remote temperature measurements. The correlation between calculated and measured (via probe) temperatures suggests that the calculated temperatures reflect that of the bulk as opposed to local to the ML bilayer. Hence, bilayer temperature could not be differentiated from bulk temperature. This finding was initially counterintuitive considering that heat originated within the MLs before being transferred through the bilayer into the bulk aqueous phase. To resolve this, a simple heat transfer analysis was adopted from Keblinski et al. [18], where they examined nanoscale versus global (bulk) EMF heating of nanoparticles. In the expression $T_{s}-T_{b}=S A R \rho_{p} r_{p}^{2} /(3 k), T_{s}$ is the nanoparticle surface temperature, $T_{b}$ is the bulk temperature, $\rho_{p}$ is the density of the encapsulated nanoparticles $\left(\mathrm{Fe}_{3} \mathrm{O}_{4}, 5.1 \mathrm{~g} \mathrm{~cm}^{-3}\right), r_{p}$ is the radius of the particle, and $k$ is the thermal conductivity of water $\left(0.6 \mathrm{~W} \mathrm{~m}^{-1}{ }^{\circ} \mathrm{C}^{-1}\right)$. For a single particle with $r_{p}=$ $160 \mathrm{~nm}$, which is near the average radius of the MLs, and using an SAR value of 100 $\mathrm{W} \mathrm{g}{ }^{-1} \mathrm{Fe}_{3} \mathrm{O}_{4}$ yields $T_{s}-T_{b}<10^{-5}{ }^{\circ} \mathrm{C}$. This is due to rapid heat dissipation from the nanoparticle surface with a timescale proportional to $r_{p}^{2} /\left(k / \rho c_{p}\right)$. Hence, it is unlikely that a lipid bilayer, even in contact with the nanoparticle surface, would not exhibit a different temperature than that of the bulk aqueous phase. Our results and analysis are in close agreement with theoretical work by Keblinski et al. [18]. 


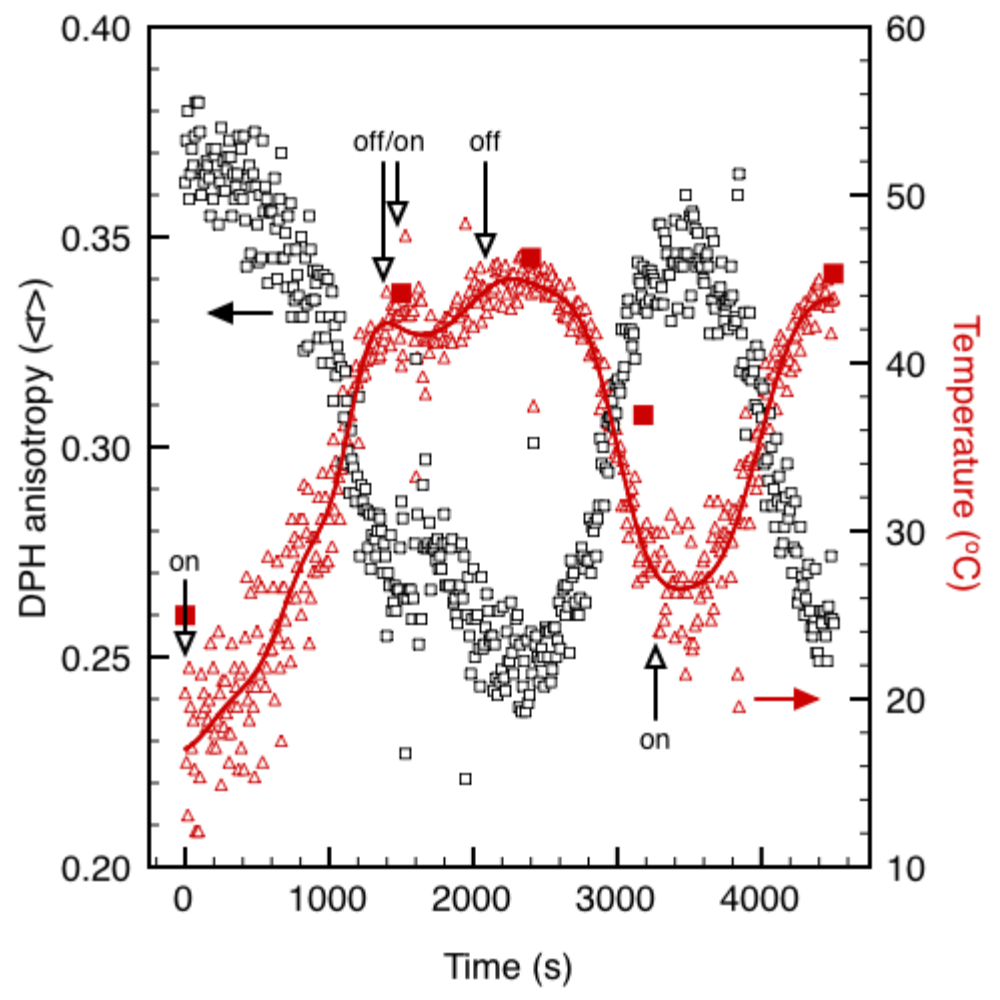

Figure 3-5. DPH anisotropy and bilayer calculated temperature with RF heating. DPH anisotropy ( $\langle r\rangle$, black squares) was measured at a RF field strength of $5 \times 10^{5} \mathrm{kA}$ $\mathrm{m}^{-1} \mathrm{~s}^{-1}$. The field was cycled randomly between off and on. Calculated temperature (red triangles) was determined from anisotropy using Eq. 1 . The results were fitted by a fourth order spline fit with an exponential correlation function, and the fitted line is intended to guide the eye and depict general trends. Temperature measurements of the bulk phase (red squares) are shown for comparison.

\section{Conclusions}

The fluorescence-based approach provides a means of measuring the lipid bilayer temperature in nanoparticle-liposome dispersions subjected to EMF heating. While the intent of this work was to provide proof-of-concept using $\mathrm{Fe}_{3} \mathrm{O}_{4}$ nanoparticles, the technique is applicable to other inorganic nanoparticles provided they do not interfere with the spectroscopic measurements. Based on the experimental results and a simple heat transfer analysis, the temperature of the bilayer in the MLs employed was equal 
to that of the bulk aqueous phase. This was due to rapid heat dissipation from the nanoparticle surface where the difference between the surface and bulk temperatures was negligible. We emphasis that in this work the MLs were not subjected to postformation processing, such as sonication, extrusion, or purification. Hence, the MLs were heterogeneous and contained unencapsulated iron oxide nanoparticles and nanoparticle aggregates. However, based on the heat transfer analysis and inferred by previous theoretical work [18], liposomal heating is not dependent on the ratio of encapsulated to unencapsulated nanoparticles, but rather on the total nanoparticle concentration within the dispersion. This suggests that nanoparticle-lipid interactions.

\section{Acknowledgements}

This work was supported by grants from the National Science Foundation (CBET0931875) and the NASA Rhode Island Space Grant Consortium. We are grateful to

Dr. Yanjing Chen for his assistance with cryo-TEM imaging and Julia Roder-Hanna for her assistance with sample preparation.

\section{References}

[1] M. Babincova, Ceska. Slov. Farm. 48 (1999) 27-29.

[2] M. De Cuyper, M. Joniau, Eur. Biophys. J. 15 (1988) 311-319.

[3] S.J.H. Soenen, M. Hodenius, M. De Cuyper, Nanomedicine 4 (2) (2009) 177-191.

[4] R. Sabate et al., Int. J. Pharm. 347 (1-2) (2008) 156-162.

[5] S. Dandamudi, R.B. Campbell, BBA-Biomembranes 1768 (3) (2007) 427-438.

[6] M. Shinkai et al., Jpn. J. Cancer Res. 87 (1996) 1179-1183. 
[7] P. Pradhan et al., J. Controlled Release 142 (1) (2010) 108-121.

[8] G. Kong, M.W. Dewhirst, Int. J. Hyperthermia 15 (5) (1999) 345-370.

[9] S. Mornet et al., J. Mater. Chem. 14 (2004) 2161-2175.

[10] P. Pradhan et al., J. Magn. Magn. Mater. 311 (2007) 208-215.

[11] I. Brezovich, Med. Phys. Monograph. 16 (1988) 82-111.

[12] E. Viroonchatapan et al., J. Control. Release 46 (3) (1997) 263-271.

[13] M. Babincova et al., Bioelectrochemistry 55 (1-2) (2002) 17-19.

[14] M. Babincova, P. Babinec, Cell Mol. Biol. Lett. 2 (1997) 3-7.

[15] M.L. Immordino, F. Dosio, L. Cattel, Int. J. Nanomed. 1 (3) (2006) 297-315.

[16] Q.A. Pankhurst et al., J. Phys. D 36 (2003) 167-181.

[17] L.A. Tai et al., Nanotechnology 20 (13) (2009).

[18] P. Keblinski et al., J. Appl. Phys. 100 (5) (2006).

[19] A. Gupta, R.S. Kane, D.A. Borca-Tasciuc, J. Appl. Phys. 108 (6) (2010).

[20] Lakowicz JR. Fluorescence anisotropy, in: J.R. Lakowicz (Ed.), Principles of

Fluorescence Spectroscopy, 2nd ed, ed., 1999, Kluwer Academic, New York.

[21] C. Bernsdorff, R. Winter, J. Phys. Chem. B 107 (38) (2003) 10658-10664.

[22] A. Samad, Y. Sultana, M. Aqil, Curr. Drug Deliv. 4 (4) (2007) 297-305.

[23] A. Wijaya, K. Hamad-Schifferli, Langmuir 23 (19) (2007) 9546-9550.

[24] F. Szoka Jr., D. Papahadjopoulos, Proc. Natl. Acad. Sci. U. S. A. 75 (9) (1978) 4194-4198.

[25] R. Koynova, M. Caffrey, Biochim. Biophys. Acta 1376 (1998) 91-145.

[26] M.D. Abramoff, P.J. Magelhaes, S.J. Ram, Biophotonics Int. 11 (7) (2004). 


\section{CHAPTER 4}

\section{Hydrophobic Nanoparticles Embedded in Liposomes Modify the Thermal}

\section{Release Behavior of Encapsulated Carboxyfluorescein}

Matthew Ryan Preiss ${ }^{1}$, Christopher Kitchens ${ }^{2}$, Ashley Hart ${ }^{2}$, and Geoffrey Bothun ${ }^{1 \dagger}$

In preparation for: ACS Nano

${ }^{1}$ University of Rhode Island, Department of Chemical Engineering,

Kingston, RI, USA

${ }^{2}$ Department of Chemical and Biomolecular Engineering, Clemson University, 130

Earle Hall, Clemson, SC, USA

${ }^{\dagger}$ Author for correspondence: Geoffrey D Bothun

Department of Chemical Engineering

University of Rhode Island

205 Crawford Hall, 16 Greenhouse Road,

Kingston, RI, 02881, USA

Phone: +1-401-874-9518

Email: gbothun@uri.edu 


\section{Abstract}

Understanding the effect of embedded nanoparticles on the characteristics and behavior of lipid bilayers is critical to the development of lipid-nanoparticle assemblies (LNAs) for biomedical applications. Decorated LNAs (D-LNAs) were formed by embedding $2 \mathrm{~nm}$ (GNP2) and $4 \mathrm{~nm}$ (GNP4) dodecanethiol-capped gold nanoparticles into DPPC liposomes at lipid to nanoparticle ratios $(\mathrm{L}: \mathrm{N})$ of 25,000:1, 10,000:1, and 5,000:1. The effect of nanoparticle size and concentration on D-LNA structure was investigated by cryogenic transmission electron microscopy, and lipid bilayer permeability and phase behavior were investigated based on the leakage of a model drug, carboxyfluorescein, and by differential scanning calorimetry, respectively. Studying leakage and phase behavior provides information on the interaction of nanoparticles on the temperature-dependent stability and permeability of liposomes. The presence of bilayer nanoparticles caused changes in the lipid bilayer release and phase behavior compared to pure lipid controls at very low nanoparticle to bilayer volume fractions $(0.3 \%-4.6 \%)$. Also, the smaller nanoparticles (GNP2) affected the bilayer differently than the nanoparticles were closer to the bilayer thickness (GNP4). By altering the bilayer phase behavior, embedded gold nanoparticles changed the bilayer permeability in a temperature-dependent fashion. 


\section{Introduction}

Liposomes are well-established biocompatible carriers capable of protecting, transporting, and delivering hydrophobic cargo (in the bilayer) and/or hydrophilic cargo (in the aqueous core) for biomedical applications. ${ }^{1,2}$ Liposomal drug delivery systems can increase therapeutic effectiveness, increase stability, target diseased sites, and control release while reducing overall toxicity and side effects ${ }^{3}$. As of 2013, the U.S. FDA has approved 13 lipid-based products for clinical use to treat cancers (such as breast, ovarian, Kaposi's sarcoma, and acute lymphoblastic leukemia), meningitis, and other ailments. They have also been approved as an anesthetic, to treat fungal infections, and for menopausal therapy. Clinical trials of lipid-based therapies are expanding especially for treatment of cancers, including colorectal, gastric, pancreatic, colon, lung, and liver cancers ${ }^{4,5}$.

One approach to controlling liposomal delivery and imparting additional functionality is through the addition of nanoparticles to form lipid-nanoparticle assemblies (LNAs) ${ }^{1-3}$. LNAs generally refer to liposome structures with embedded, encapsulated or adhered nanoparticles. For more extensive background on the formation, applications, and types of LNAs see reviews by Al-Jamal and Kostarelos ${ }^{3}$ and Bothun et al. ${ }^{1,2}$ Decorated LNAs (D-LNAs) formed with hydrophobic nanoparticles embedded in the acyl tail region of the lipid bilayer represent a unique hybrid structure where the nanoparticles add functionality and can be used to control liposomal release. Nanoparticles used to form D-LNAs must be similar in size to the thickness of the lipid bilayer (4-5 nm) in order to embed. Theoretically, the lipid bilayer can distort to accommodate nanoparticles up to $6.5 \mathrm{~nm}^{6,7}$. Stable D-LNAs 
have been formed with iron oxide ${ }^{8,9}$, quantum dots $(\mathrm{CdSe} \text { and } \mathrm{ZnS})^{10-14}$, gold ${ }^{14-18}$, silver $^{19,20}$, silicon ${ }^{21}, \mathrm{C}_{60}$ fullerenes ${ }^{22,23}$, and cobalt ferrite ${ }^{24}$ nanoparticles giving them targeting, controlled release, diagnostic, and stimuli-responsive capabilities.

Recent D-LNA research has focused on forming and characterizing D-LNA structures or developing D-LNAs with new functionalities. Structural D-LNA research has mainly focused on increasing nanoparticle concentrations, incorporating larger nanostructures and/or nanoparticle aggregates, and nanoparticle localization and mobility within the bilayers ${ }^{8,15,16,25-28}$. Functional D-LNA research has led to the development of stimuli-responsive, targeting, imaging, diagnostic, therapeutic, and/or controlled release capabilities for biomedical applications ${ }^{8-11,13,18}$. However, few studies have been conducted to determine the effects of embedded nanoparticles on lipid phase behavior, notably the pretransition $\left(\mathrm{T}_{\mathrm{p}}\right)$ and melting $\left(\mathrm{T}_{\mathrm{m}}\right)$ temperatures, and the associated thermal release behavior. Furthermore, studies that have been reported suggest that nanoparticle size and concentration can lead to stabilizing or destabilizing effects based on lipid ordering and phase behavior. These properties are important when designing D-LNAs for drug delivery applications; and a deeper understanding of the relationship between D-LNA structure, phase behavior, and release properties could be used to design D-LNAs a priori for specific applications.

Oh et al. have shown that the membrane fluidity above $\mathrm{T}_{\mathrm{m}}$ of Dipalmitoylphosphatidylcholine (DPPC) increased with increased loading of $4 \mathrm{~nm}$ nanoparticles, consistent with liposome destablization ${ }^{17,19}$. Similarly, Bothun et al. have observed that increasing the concentration of $5.7 \mathrm{~nm}$ nanoparticles reduced the $\mathrm{T}_{\mathrm{p}}, \mathrm{T}_{\mathrm{m}}$, and lipid ordering of DPPC D-LNAs ${ }^{20}$. In contrast, liposome stabilization was 
observed by Chen et. al. where embedded $5 \mathrm{~nm}$ nanoparticles suppressed leakage by stabilizing the bilayer based on increases in $\mathrm{T}_{\mathrm{m}}$ with increasing nanoparticle loading ${ }^{8}$. This behavior was also observed by Von White et al. where $3.9 \mathrm{~nm}$ gold nanoparticles and excess stabilizing ligand could significantly increase $T_{m}$ at high nanoparticle loadings ${ }^{15}$.

The aim of this research is to investigate the effect of hydrophobic nanoparticle size and concentration on the structure, leakage, and phase behavior of D-LNAs. DPPC (1,2-dipalmitoyl-sn-glycero-3-phosphocholine) bilayers were loaded with dodecanthiol-coated gold nanoparticles (GNPs) of different sizes and lipid-tonanoparticle (L:N) ratios. D-LNAs were loaded with $2 \mathrm{~nm}$ (GNP2) and $4 \mathrm{~nm}$ (GNP4) gold nanoparticles. Figure 4-1 depicts the likely D-LNA structures formed from DPPC and GNP2 and GNP4, and the structure of DPPC and associated thermal phase behavior. Figure 4-1 also depicts nanoparticle clusters in the bilayer of a D-LNA. This study was possible by fractionating the AuNPs into discrete size fractions using a high-pressure carbon dioxide-based antisolvent process. The interaction between the nanoparticles and the lipid bilayer was investigated with cryogenic transmission electron microscopy (cryo-TEM), fluorescent leakage of a model drug (carboxyfluorescein or CF), and differential scanning calorimetry (DSC). Our results show that GNPs influence the phase behavior of DPPC liposomes, which in turn modifies the thermal CF release behavior. 


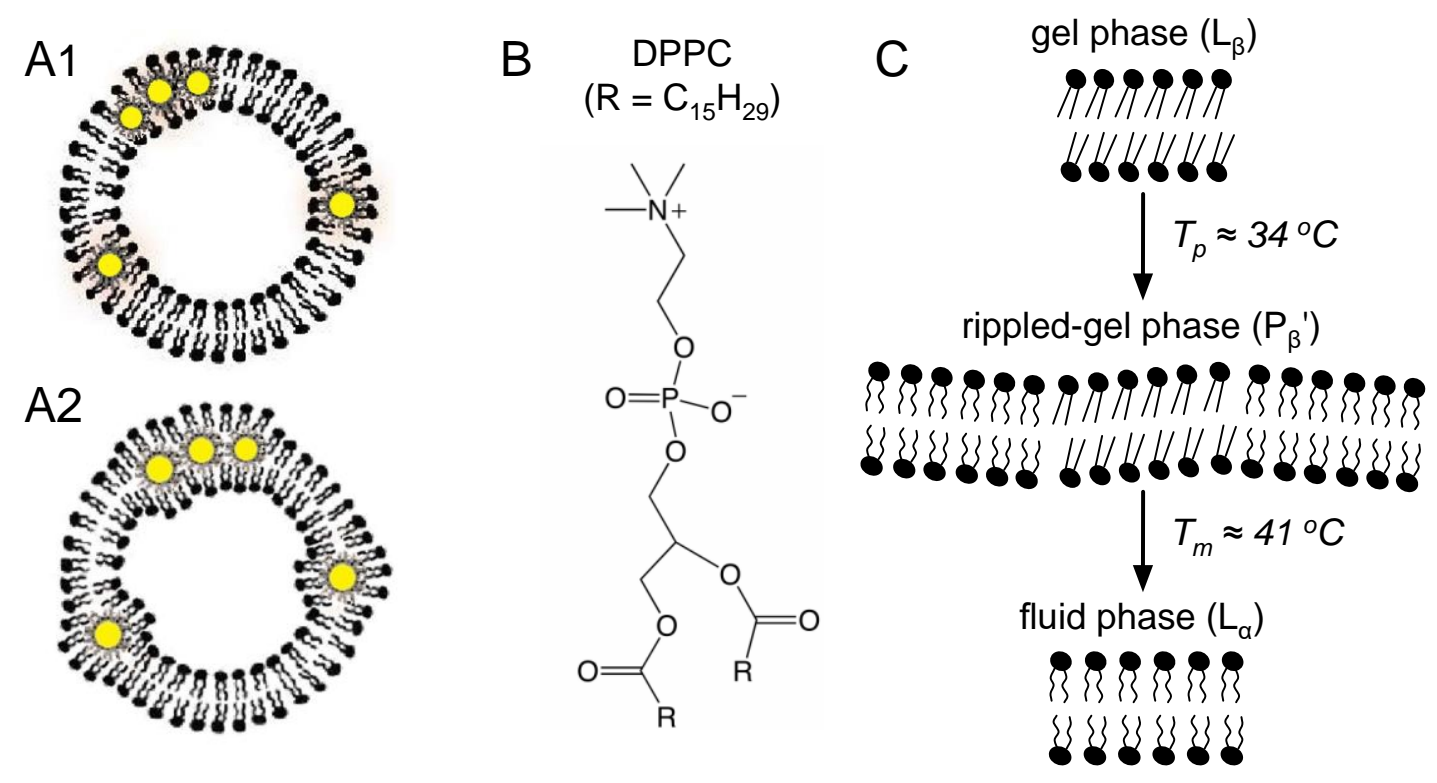

Figure 4-1. Schematics of D-LNA structures loaded with GNP2 and GNP4.

(A) GNP2 are assimilated without much bilayer deformation because GNP2 are smaller than the thickness of a DPPC lipid bilayer $(4-5 \mathrm{~nm}) .{ }^{6}$ (B) GNP4 cause the lipid bilayer to thicken around the nanoparticle. ${ }^{15}(\mathrm{~B})$ Structure and $(\mathrm{C})$ phase behavior of DPPC with associated pretransition $\left(\mathrm{T}_{\mathrm{p}}\right)$ and main or melting transition $\left(\mathrm{T}_{\mathrm{m}}\right)$ temperatures.

\section{Methods and Materials}

\subsection{Chemicals and Materials.}

Dipalmitoylphosphatidylcholine (DPPC) in chloroform $(25 \mathrm{mg} / \mathrm{mL})$ was purchased from Avanti Polar Lipids (Alabaster, AL). 5,6-Carboxyfluorescein (CF) and Triton X-100 were purchased from Sigma Aldrich (St. Louis, MO). Phosphate buffered saline 1X solution (PBS) was purchased from Fisher Scientific (Suwanee, GA). Sterile deionized ultrafiltered (DI) water at $18.2 \mathrm{~m} \Omega$ was used from a Millipore Direct-Q3 UV purification system (Billerica, MA). 


\subsection{Gold Nanoparticle Synthesis.}

Dodecanethiol-stabilized gold nanoparticles (DDT-GNPs) were synthesized via an arrested precipitation method previous described for silver ${ }^{29}$, then modified for gold. ${ }^{16}$ In short, $330 \mathrm{mg}$ of gold chloride trihydrate (Acros Organics, 99\%) was dissolved in $20 \mathrm{~mL}$ of DI water and $6 \mathrm{~g}$ of tetraoctylammonium bromide, TOAB, (Chem-Impex Int'1 Inc, 99.35\%) was dissolved in $80 \mathrm{~mL}$ of chloroform (Alfa Aesar, HPLC grade 99.5\%). The two solutions were then combined and stirred vigorously for 1 hour until the chloroform phase become an orange/red color. The aqueous phase was discarded and $600 \mu \mathrm{L}$ of dodecanethiol, DDT, (Tokyo Chemical Industry, >95\%) was added to the gold/chloroform solution and stirred for 30 minutes. $20 \mathrm{~mL}$ of $0.5 \mathrm{M}$ sodium borohydride was then added to the gold/DDT/chloroform mixture and stirred for 12 hours. The aqueous phase was then discarded and the nanoparticle solution was then washed with methanol (Burdick \& Jackson, HPLC grade) to remove any excess DDT and TOAB and resuspended in neat toluene (BDH, 99.5\%).

\subsection{Nanoparticle Fractionation.}

The nanoparticle synthesis process results in a polydisperse sample comprised of nanoparticles with diameters ranging from 1-10 nm. For monodisperse nanoparticle samples large (diameters of $4 \mathrm{~nm}$ ) or small (diameters less than $3 \mathrm{~nm}$ ) nanoparticles, nanoparticle fractionation using a solvent/antisolvent centrifugation method (as described in Korgel et. al. ${ }^{16}$ ) was used to separate the nanoparticles by size. During the fractionation process, the largest nanoparticles precipitate out during centrifugation with the supernatant comprised of the smaller nanoparticles. Fractionations are 
performed with different volume percentages of antisolvent (methanol). The first fractionation is performed with $40 \%$ antisolvent or $40 \% \mathrm{v} / \mathrm{v}$ methanol. The nanoparticle solution is mixed with the $40 \%$ antisolvent and vortexed. The methanol/Au NP mixture is centrifuged at $14500 \mathrm{rpm}$ for 10 minutes. The precipitate (with nanoparticles $\sim 4-10 \mathrm{~nm}$ in diameter) is resuspended in toluene or chloroform. Large and small nanoparticles remain in the supernatant. The supernatant is transferred to a new centrifuge tube and the process is repeated for the second fractionation at $70 \%$ antisolvent. The precipitate, consisting of large nanoparticles (4 $\mathrm{nm}$ nanoparticles), is resuspended in toluene or chloroform. Small nanoparticles (nanoparticle diameters less than $3 \mathrm{~nm}$ ) remain in the supernatant, which is retained for the final fractionation with $90 \% \mathrm{v} / \mathrm{v}$ methanol. The solvent/antisolvent mixture is removed by rotary evaporation and then is resuspended in toluene or chloroform.

\subsection{Nanoparticle Characterization.}

Transmission electron microscopy (TEM) was used to determine the size distribution of each nanoparticle fraction. After fractionation and redispersement in neat solvent, a drop of the nanoparticle solution was placed on a 400 mesh Formvar/carbon coated copper grid (Electron Microscopy Sciences) and dried in air. The TEM measurements were conducted on a Hitachi 7600 instrument operating at an acceleration voltage of $120 \mathrm{kV}$. The size distributions were analyzed using ImageJ software. For GNP2, size was determined by manually measuring 50 random particles. For GNP4, size was determined by the automatic measurement of 500-1000 particles. 
Dynamic Light Scattering (DLS) was also used to study the size of each nanoparticle fraction. Nanoparticle solutions were placed in clear, scratch free scintillation vials for DLS measurement with a Wyatt Technologies Dawn Heleos II with the QELS option. First, a nanoparticle free solvent, in this case toluene, was analyzed to subtract the scattering of the solvent from the scattering pattern of the nanoparticles. Each fraction was analyzed separately and the nanoparticle free solvent was analyzed again after each fraction to establish a baseline. The sample time for each fraction and background measurement was 5 minutes. It is important to note that the size obtained by DLS also includes the solvent-ligand saturation layer around the nanoparticle and therefore will differ from TEM size results.

\subsection{Liposome and DLNA Preparation.}

$10 \mathrm{mM}$ DPPC control liposomes and $10 \mathrm{mM}$ DPPC DLNAs with lipid molecule to nanoparticle (L:N) ratios of 25000:1, 10000:1, and 5000:1 were formed by thin film hydration. Lipids and GNPs in chloroform were mixed in a $25 \mathrm{~mL}$ round bottom flask. Chloroform was removed by a BÜCHI Rotavapor R-215 rotoary evaporation (Flawil, Switzerland) at $50^{\circ} \mathrm{C}$ (greater than the melting temperature of DPPC) at $300 \mathrm{mbar}$ for $30 \mathrm{~min}$ and then $50 \mathrm{mbar}$ for $10 \mathrm{~min}$. To evaporate residual chloroform forming a thin film, the flask was placed in a vacuum at greater than 25 in Hg overnight. For release studies, the thin film was rehydrated with 1X PBS containing $50 \mathrm{mM} \mathrm{CF}$ in the flask. The flask was sonicated in a $40 \mathrm{kHz}$ Branson Ultrasonics 1510 ultrasonic cleaner (Danbury, CT, USA) a $50^{\circ} \mathrm{C}$ bath for $60 \mathrm{~min}$. while rotating. All liposomes and D-LNA samples had broad diameter ranges typically 
between 40-125 nm. Overall, the largest and the smallest liposome and D-LNA structures observed were $266 \mathrm{~nm}$ and $39 \mathrm{~nm}$ in diameter, respectively.

Unencapsulated CF was removed by a modified dry size exclusion chromatography [30]. Chromatography columns were prepared by loading $0.5 \mathrm{~g}$ of Sephadex G-50 (medium) mixed in PBS. Columns were centrifuged in a Thermo Fisher Scientific Heraeus Megafuge 16R (Waltham, MA, USA) at 1000 XG for 3 min. removing the PBS and leaving a chromatography column packed with Sephadex. The liposome or DLNA sample with unencapsulated CF is added to the dry Sephadex column and centrifuged for $100 \mathrm{XG}$ for $10 \mathrm{~min}$. then $1000 \mathrm{XG}$ for $3 \mathrm{~min}$. removing the unencapsulated CF. The sample that flows through the Sephadex column is collected (repeated 2 times).

\subsection{Fluorescence Leakage Studies.}

CF leakage experiments were conducted within a PerkinElmer LS 55 fluorescence spectrometer with a PTP 1 Peltier Temperature Programmer for temperature control (Waltham, MA, USA). Fluorescence measurements were taken at excitation and emission wavelengths of 492 and $517 \mathrm{~nm}$, respectively, a $5 \mathrm{~nm}$ excitation and emission slit width, and an integration time of $10 \mathrm{sec} .2 \mu \mathrm{L}$ of DPPC control or DLNA was added to $3 \mathrm{~mL}$ of $1 \mathrm{X}$ PBS in a quartz cuvette constantly mixing with a magnetic stir bar. CF intensity readings of the samples were taken as a function of temperature between $25^{\circ} \mathrm{C}$ and $46{ }^{\circ} \mathrm{C}$ at increments of $1{ }^{\circ} \mathrm{C}$. Each temperature was maintained for $5 \mathrm{~min}$. After the temperature readings, $10 \mu \mathrm{L}$ of $2 \%$ Triton $\mathrm{X}-100$ was 
added to the cuvette and the cuvette was sonicated to lyse the liposomes or DLNAs. The percentage of $\mathrm{CF}$ leakage was calculated from the following equation:

$$
C F \text { Leakage } \%=100 \times \frac{I(T)-I_{0}}{I_{f}-I_{0}}
$$

where $I(T)$ is the CF intensity at temperature $\mathrm{T}, I_{0}$ is the initial CF intensity, and $I_{f}$ is the CF intensity after lysing. All leakage studies were performed in triplicate.

CF intensity readings were also taken as a function of time at constant temperature, 25

${ }^{\circ} \mathrm{C}$ and $45^{\circ} \mathrm{C}$, corresponding to the DPPC liposomes being in the gel phase and fluid phase, respectively. $2 \mu \mathrm{L}$ of DPPC control or D-LNA was added to $3 \mathrm{~mL}$ of $1 \mathrm{X}$ PBS in a quartz cuvette constantly mixing with a magnetic stir bar. A baseline CF intensity, $\mathrm{I}_{0}$, was recorded for $5 \mathrm{~min}$. The temperature was changed and CF intensity was integrated and recorded every $10 \mathrm{sec}$. for $30 \mathrm{~min}$., I(t). After $30 \mathrm{~min}, 10 \mu \mathrm{L}$ of $2 \%$ Triton X-100 was added to the cuvette to lyse the DPPC liposomes or D-LNAs. Intensity was recorded for another 15 min to determine the intensity of all the CF in the sample, If. Using these terms, percent CF leakage was calculated with the following equation:

$$
\text { CF Leakage } \%=100 \times \frac{I(t)-I_{0}}{I_{f}-I_{0}}
$$

For all CF quenching experiments, the quenching was not affected by the gold nanoparticles because the $\mathrm{CF}$ quenching was normalized and they reside in the lipid bilayer preventing it from affecting CF quenching. 


\subsection{Differential Scanning Calorimetry.}

Lipid phase behavior was investigated with a TA Instruments Nano DSC (New

Castle, DE, USA). DPPC and D-LNA samples were diluted in 1xPBS to $0.5 \mathrm{mM}$ lipid. Samples and 1xPBS were degassed and loaded into the DSC's $0.76 \mathrm{~mL}$ sample and reference capillary cells, respectively. The DSC cell was pressurized to 3 atm and equilibrated at $20^{\circ} \mathrm{C}$. A heating cycle from $20-50{ }^{\circ} \mathrm{C}$ at scan rate of $1{ }^{\circ} \mathrm{C} \min ^{-1}$ with a 5 min equilibration period at $20^{\circ} \mathrm{C}$ and $50{ }^{\circ} \mathrm{C}$ were performed.

\subsection{Cryogenic Transmission Electron Microscopy (Cryo-TEM).}

DPPC liposome and D-LNA sizes and structures were determined with cryoTEM imaging. $\sim 5 \mu \mathrm{L}$ of sample is deposited on a Quantifoil grid comprised of 200 square mesh copper grids suspended with $2 \mu \mathrm{m}$ carbon hole. (Electron Microscopy Sciences, Hatfield, PA, USA). Grids were robotically vitrified in liquid ethane using a Vitrobot (FEI Company). Prior to imaging, the vitrified grid is transferred and stored in liquid nitrogen. Imaging was performed in a liquid nitrogen cooled stage (Model 915, Gatan Inc., Pleasonton, CA) at $200 \mathrm{kV}$ using a JEOL JEM-2100F TEM (Peabody, MA). DPPC liposome and D-LNA size analysis was performed using ImageJ software [31]. The average diameters and standard deviations reported were based on 92 randomly selected liposomes or D-LNAs.

\section{Results and Discussion}

D-LNAs were prepared with two different size fractions of gold nanoparticles; fraction GNP2 had an average diameter \pm S.D. of $1.99 \pm 0.5 \mathrm{~nm}$ (Figure 4-2A) and 
fraction GNP4 had an average diameter \pm S.D. of $4.01 \pm 0.8 \mathrm{~nm}$ (Figure 4-2B). The nanoparticles were loaded into the liposomal bilayers at lipid:nanoparticle (L:N) ratios of 25,000:1, 10,000:1, and 5,000:1. GNP concentrations and calculated volume fractions within the D-LNAs are provided in Table 4-1.

DPPC liposomes and D-LNAs were characterized by cryo-TEM (Figure 4-3). DPPC liposomes were primarily spherical unilamellar structures with an average diameter of $101 \pm 45 \mathrm{~nm}$ (Figure 4-3A) D-LNAs formed with GNP2 and GNP4 had average diameters of $56 \pm 21 \mathrm{~nm}$ (Figure 4-3B, C) and $69 \pm 36 \mathrm{~nm}$ (Figures 4-3D, E, F1, and F2), respectively. In addition to reducing the liposome diameter, GNP loading led to less spherical structures with faceted edges and thicker bilayers. GNP4 yielded liposomes containing nanoparticle clusters within the bilayers; this likely also occurred for GNP2 based on previous work, however, we could not confirm this. It should be noted that direct evidence of nanoparticle loading was difficult to obtain at the high L:N ratios used. As demonstrated by recent D-LNA studies, nanoparticle loading can be more easily observed at significantly lower L:N ratios or higher

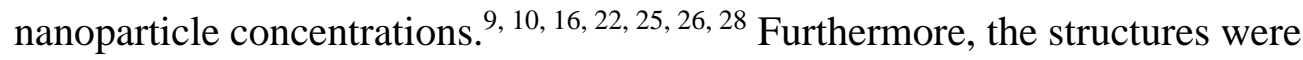
heterogeneous, with some D-LNAs containing GNP clusters that are clearly visible (Figure 4-3D, F1-F2) and others were GNPs were not directly observed. There were also 'darker' D-LNAs that suggest they were loaded with GNPs, but the individual GNPs could not be resolved. We believe that the difficulty in identifying bilayerembedded GNPs stems in part from the limitations experienced during cryo-TEM imaging. This is depicted in Figure 4-3F1-F2 where a D-LNA with a 'dark spot' is observed, but only when the TEM is over-focused do we see that this spot is 
comprised of a GNP cluster. This does not mean that all structures in the D-LNA samples contained embedded GNPs - it is likely that there were also 'empty' liposomes present. Additional TEM work is needed to confirm these assertions.
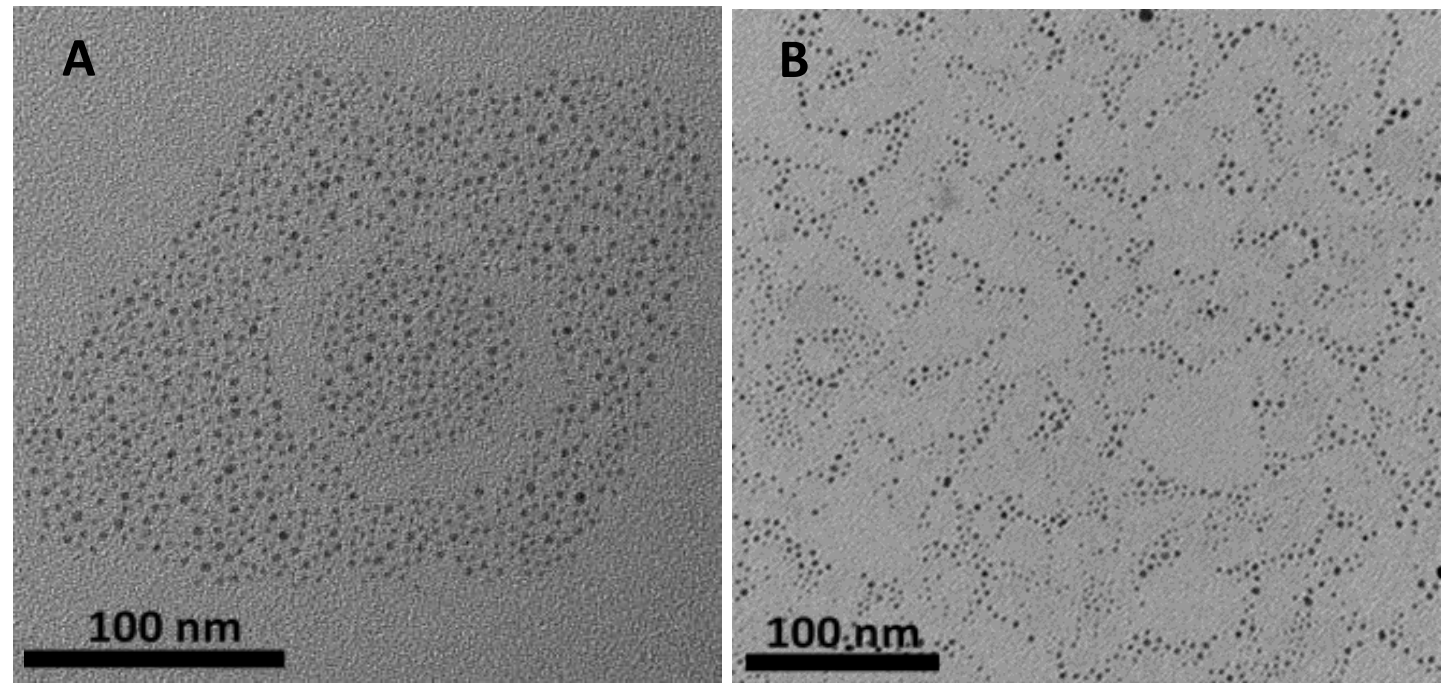

Figure 4-2. TEM Images of GNPs.

(A) GNP2 had an average diameter \pm S.D. of $1.99 \pm 0.5 \mathrm{~nm}$ and (B) GNP4 had an average diameter \pm S.D. of $4.01 \pm 0.8 \mathrm{~nm}$.

Table 4-1. Concentration and volume fraction of GNPs loaded into D-LNAs.

\begin{tabular}{cccccc}
\hline \multirow{2}{*}{ GNP fraction } & L:N & \multicolumn{2}{c}{ Concentration } & \multicolumn{2}{c}{ GNP Volume Fraction } \\
& & $(\mathbf{m g} / \mathbf{m l})$ & $(\mathbf{m M})$ & Gel $^{\mathbf{b}}$ & Fluid $^{\mathbf{c}}$ \\
\hline \multirow{2}{*}{ GNP2 } & $25,000: 1$ & 0.02 & 0.10 & $0.4 \pm 0.1 \%$ & $0.4 \pm 0.1 \%$ \\
$(1.99 \pm 0.5 \mathrm{~nm})$ & $10,000: 1$ & 0.05 & 0.25 & $1.1 \pm 0.3 \%$ & $1.0 \pm 0.3 \%$ \\
& $5,000: 1$ & 0.10 & 0.50 & $2.2 \pm 0.6 \%$ & $2.0 \pm 0.6 \%$ \\
\hline GNP4 & $25,000: 1$ & 0.16 & 0.8 & $1.1 \pm 0.4 \%$ & $1.0 \pm 0.4 \%$ \\
$(4.01 \pm 0.8 \mathrm{~nm})$ & $10,000: 1$ & 0.40 & 2.0 & $2.8 \pm 1.0 \%$ & $2.5 \pm 0.9 \%$ \\
& $5,000: 1$ & 0.79 & 4.0 & $5.6 \pm 2.0 \%$ & $5.1 \pm 1.8 \%$ \\
\hline
\end{tabular}

${ }^{\text {a} B a s e d ~ o n ~ n a n o p a r t i c l e ~ d i a m e t e r ~ w i t h ~} 1.8 \mathrm{~nm}$ (fully extended) DDT ligands ${ }^{32}$.

${ }^{b}$ Volume fraction in the hydrocarbon acyl region of gel phase DPPC bilayers (825 $\AA^{3} /$ lipid $)^{33}$.

${ }^{c}$ Volume fraction in the hydrocarbon acyl region of fluid phase DPPC bilayers (913 $\AA^{3} /$ lipid $)^{33}$. 

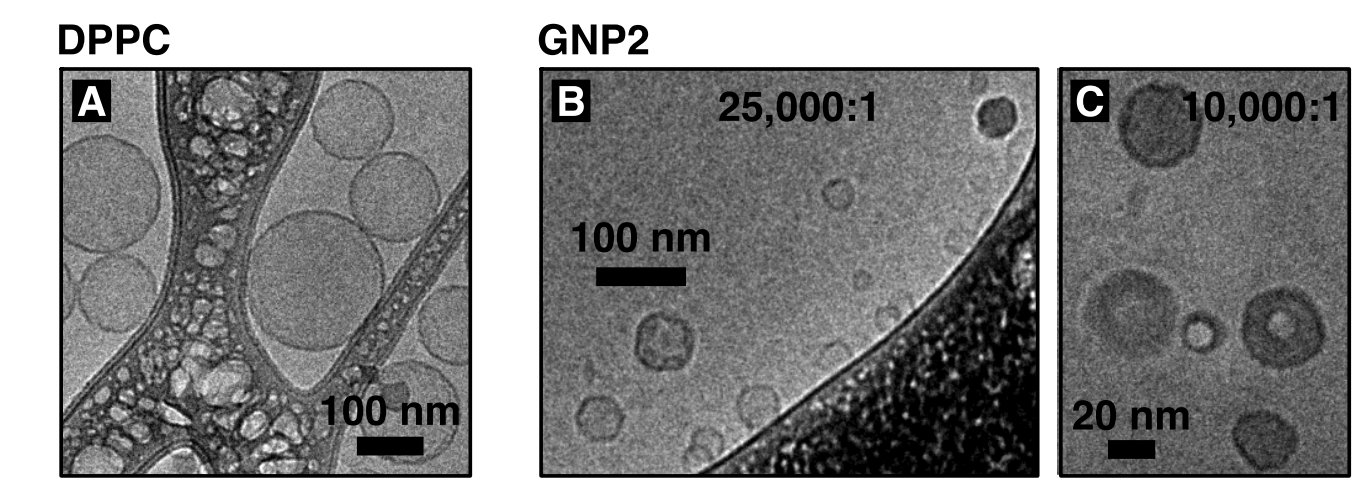

\section{GNP4}
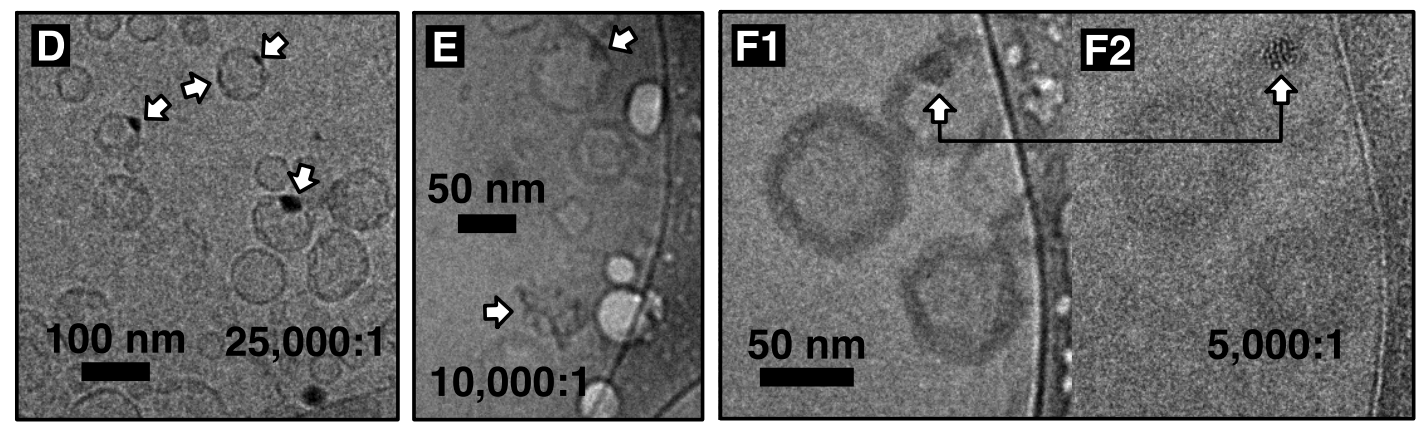

Figure 4-3. Representative Cryo-TEM micrographs.

(A) DPPC liposomes, (B-C) D-LNAs prepared with GNP2, and (D-F) D-LNAs prepared with GNP4. The L:N ratio is shown on each micrograph. (F1-F2) are micrographs of the same region with different focus, which was able to identify the individual GNPs that comprised the GNP aggregate contained within the D-LNA bilayer. White arrows identify the location of embedded nanoparticles that can be viewed directly.

Additional analyses were performed on D-LNAs prepared with GNP4 at a higher L:N ratio of 1,000:1 to confirm that GNPs were loaded into the bilayers. CF leakage was not examined at this higher L:N ratio. D-LNAs loaded with GNPs were easily visible by cryo-TEM analysis (Figure 4-4A1, B1). Magnified images of DLNAs with slight over-focusing revealed that the GNPs were disordered within the bilayers (Figure 4-4A2, B2). FE-SEM analysis with EDS confirmed that the structures were loaded with GNPs (Figure 4-4C1, C2). In this case gold is concentrated where DLNAs are observed. 

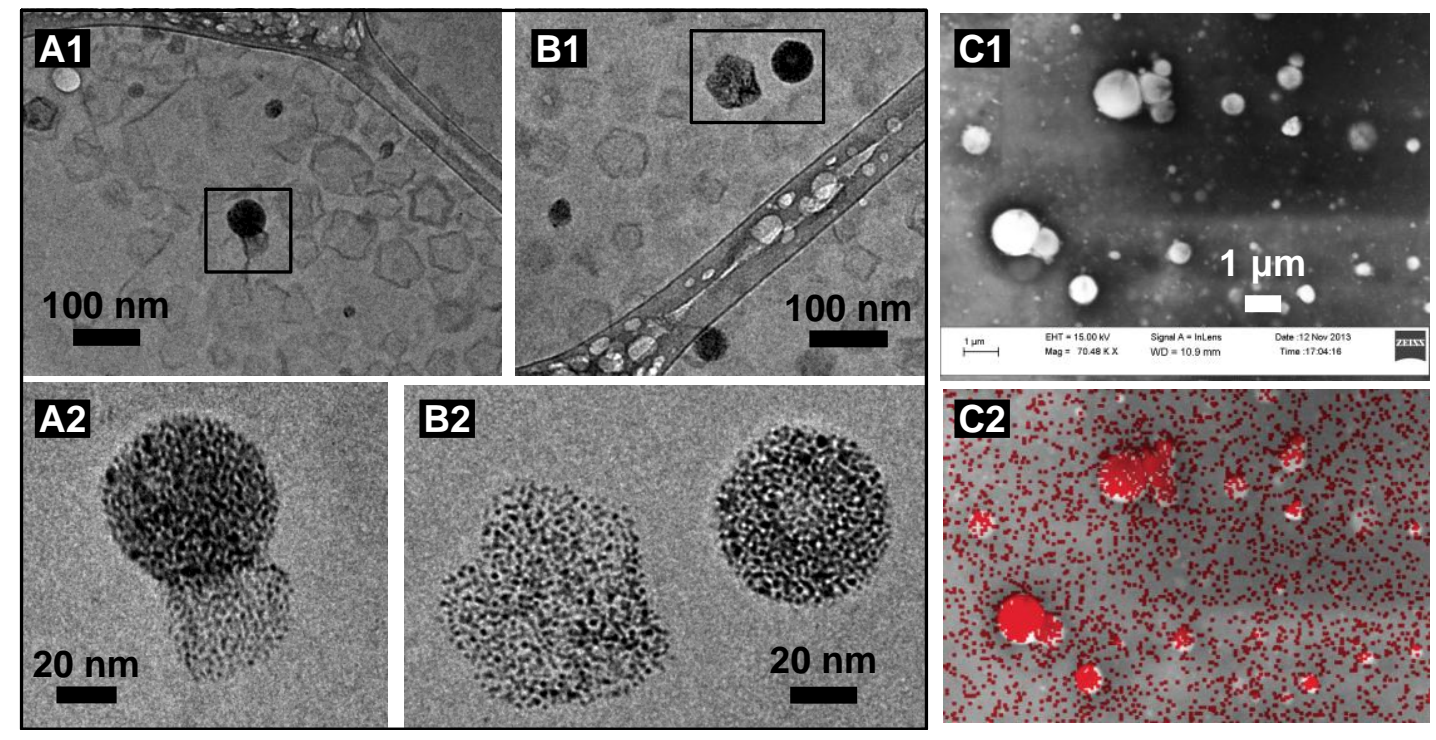

Figure 4-4. Representative Cryo-TEM and FE-SEM of GNP4 D-LNAs at Lipid:Nanoparticle Ratio of 1000:1.

Cryo-TEM (A, B) and FE-SEM (C1) micrographs of D-LNAs prepared with GNP4 at $\mathrm{L}: \mathrm{N}=1,000: 1$. Magnified and over-focused regions from A1 and B1 are shown in A2 and B2, respectively. (C2) EDS map of gold obtained from image C1.

\section{Carboxyfluoroscein (CF) leakage from DPPC liposomes and D-LNAs was}

measured in triplicate as a function of temperature from $26{ }^{\circ} \mathrm{C}$ to $47{ }^{\circ} \mathrm{C}$. This

temperature range spanned the pretransition $\left(\mathrm{T}_{\mathrm{p}}=34.4^{\circ} \mathrm{C}\right)$ and melting temperatures

$\left(\mathrm{T}_{\mathrm{m}}=41.3^{\circ} \mathrm{C}\right)$ of $\mathrm{DPPC}^{34}$. DPPC liposomes showed increases in CF leakage between

$32{ }^{\circ} \mathrm{C}$ and $36{ }^{\circ} \mathrm{C}$, corresponding to the gel to rippled gel pretransition, $\left(\mathrm{L}_{\beta} \rightarrow \mathrm{P}_{\beta}{ }^{\prime}\right)$, and between $38{ }^{\circ} \mathrm{C}$ and $41{ }^{\circ} \mathrm{C}$, corresponding to the rippled gel to fluid or melting transition $\left(\mathrm{P}_{\beta}{ }^{\prime} \rightarrow \mathrm{L}_{\alpha}\right)$ (Figure 4-5). The $\mathrm{CF}$ leakage behavior reflects the two bilayer phase transitions that occur within the temperature range examined. Comparatively little change in CF leakage was observed when the liposomes were in their gel $(<32$ $\left.{ }^{\circ} \mathrm{C}\right)$ or fluid $\left(>41{ }^{\circ} \mathrm{C}\right)$ phases, consistent with previous studies. ${ }^{35}$ 
CF leakage from D-LNAs prepared with GNP2 exhibited a 'temperature lag' where leakage shifted to higher temperatures and the total amount of CF leakage at 47 ${ }^{\circ} \mathrm{C}$ reduced by roughly 50\% when compared to DPPC (Figure 4-5A). CF leakage increased between $35^{\circ} \mathrm{C}$ and $42^{\circ} \mathrm{C}$, after which a modest linear increase in $\mathrm{CF}$ leakage was observed above $41^{\circ} \mathrm{C}(<5 \%$, Figure $4-5 \mathrm{~A})$. There was no clear trend between the CF leakage and GNP2 loading (L:N).

D-LNAs prepared with GNP4 exhibited different CF leakage behavior compared to GNP2. At the two lower GNP loadings (L:N = 25,000:1 and 10,000:1), increases in $\mathrm{CF}$ leakage were observed between $34{ }^{\circ} \mathrm{C}$ and $36{ }^{\circ} \mathrm{C}$, and between $39{ }^{\circ} \mathrm{C}$ and $42{ }^{\circ} \mathrm{C}$ (Figure 4-5B). The 'temperature lag' for $\mathrm{CF}$ leakage was reduced with the larger GNPs (compared to GNP2) and the leakage behavior reflected the phase transitions that were observed for DPPC, though the absolute leakage was not as large. At $\mathrm{L}: \mathrm{N}=5,000: 1$ there was no 'temperature lag' and CF leakage increased linearly from $26{ }^{\circ} \mathrm{C}$ to $40{ }^{\circ} \mathrm{C}$, with no additional leakage above $43{ }^{\circ} \mathrm{C}$. Based on the $\mathrm{CF}$ leakage behavior, GNP2 had a greater effect on stabilizing the bilayer and reducing bilayer permeability compared to GNP4. This is consistent with the ability of liposome bilayers to accommodate smaller nanoparticles. 

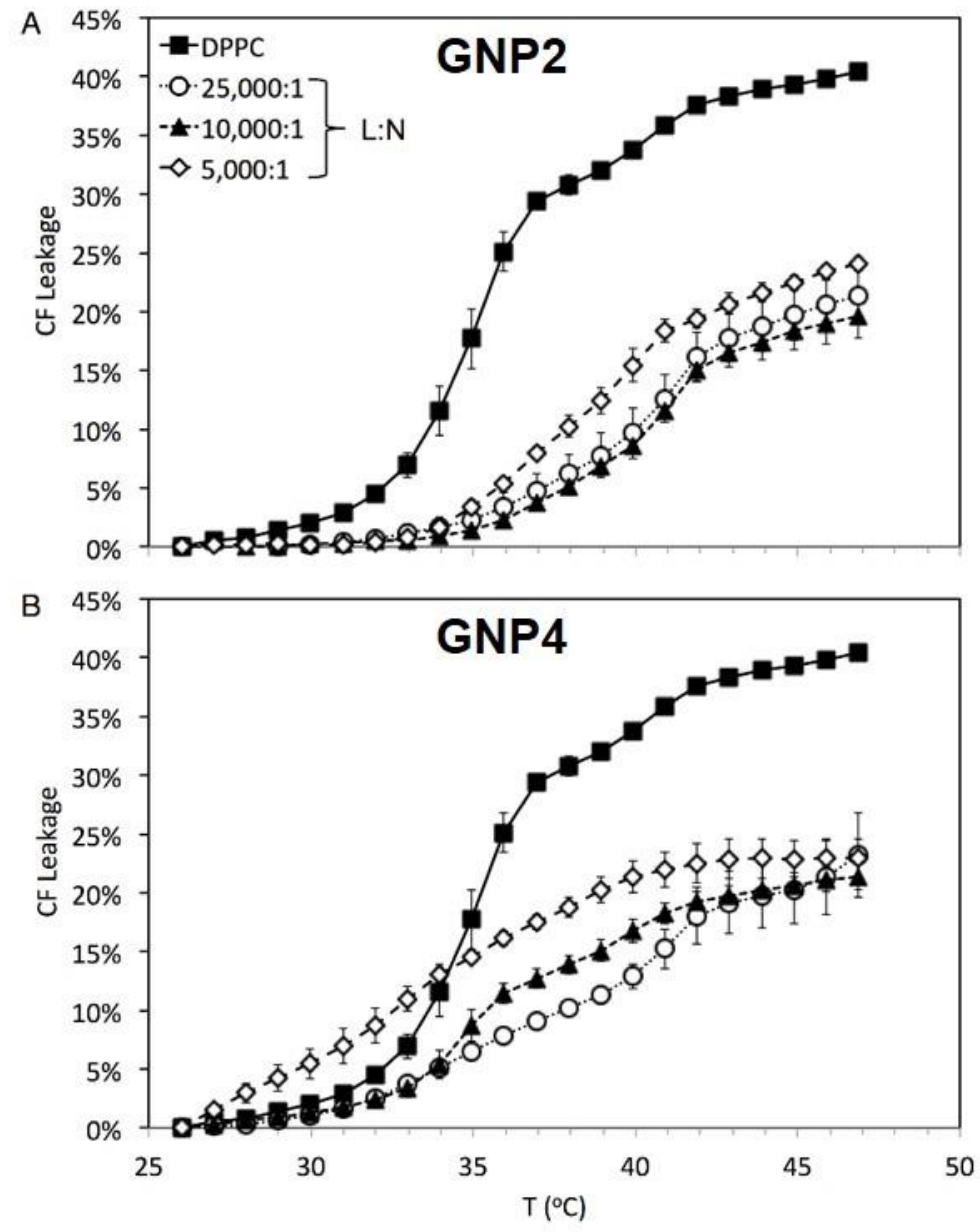

Figure 4-5. Percentage of carboxyfluorecein (CF) leakage as a function of temperature.

DPPC liposomes and D-LNAs prepared with nanoparticle fractions (A) GNP2 and (B) GNP4. The symbols and error bars correspond to the average \pm S.D.

Results from CF leakage suggest that the leakage behavior is dependent upon bilayer phase behavior and the degree to which this changes in the presence of the GNPs. To examine this more closely, the derivative of CF leakage as a function of temperature was calculated numerically based on $\Delta \mathrm{CF}$ leakage $/ \Delta \mathrm{T}$ to determine the temperatures associated within maximum and minimum changes in $\mathrm{CF}$ leakage, and to directly compare changes in CF leakage with D-LNA phase behavior determined by 
DSC. DSC results are summarized in Table 4-2. The The pretransition and melting transition appear as peaks in the excess heat capacity $\left(C_{p}\right.$, Figure 4-6). ${ }^{33,34,36}$

Table 4-2. DPPC and D-LNA lipid phase behavior and CF leakage temperatures.

\begin{tabular}{ccccccc}
\hline $\begin{array}{c}\text { GNP } \\
\text { Fraction }\end{array}$ & $\mathbf{L : N}$ & $\mathbf{T}_{\mathbf{p}}\left({ }^{\circ} \mathbf{C}\right)^{\mathbf{a}}$ & $\mathbf{T}_{\mathbf{m}, \mathbf{o n}}\left({ }^{\circ} \mathbf{C}\right)^{\mathbf{b}}$ & $\mathbf{T}\left({ }^{\circ} \mathbf{C}\right)^{\mathbf{a}}$ & $\mathbf{T}_{\mathbf{C F}}\left({ }^{\circ} \mathbf{C}\right)^{\mathbf{c}}$ & $\Delta_{\mathbf{1 / 2}}\left({ }^{\circ} \mathbf{C}\right)^{\mathbf{d}}$ \\
\hline DPPC & & 35.8 & 40.4 & 41.1 & $\mathbf{3 4 . 5}, 39.5$ & 0.9 \\
\hline GNP2 $^{\mathrm{b}}$ & $10,000: 1$ & 36.0 & 40.7 & 41.2 & 40.5 & 0.9 \\
& $5,000: 1$ & 36.3 & 40.6 & 41.2 & $35.5, \mathbf{3 8 . 5}$ & 1.0 \\
\hline & $25,000: 1$ & $35.6^{\mathrm{e}}$ & 39.4 & 40.8 & $32.5, \mathbf{4 0 . 5}$ & 1.6 \\
GNP4 $^{\mathrm{b}}$ & $10,000: 1$ & $35.6^{\mathrm{e}}$ & 39.1 & 40.6 & $\mathbf{3 3 . 5}, 38.5$ & 1.8 \\
& $5,000: 1$ & $-^{\mathrm{e}}$ & 40.2 & 41.5 & $\mathbf{3 1 . 5}, 37.5$ & 1.5 \\
\hline
\end{tabular}

${ }^{\mathrm{a}} \mathrm{T}_{\mathrm{p}}$ and $\mathrm{T}_{\mathrm{m}}$ correspond to maximum $\mathrm{C}_{\mathrm{p}}$.

${ }^{\mathrm{b}}$ The melting onset temperature $\left(\mathrm{T}_{\mathrm{m}}\right.$, on $)$ is the temperature at which the rippled gel to fluid main transition begins.

${ }^{\mathrm{c}} \mathrm{T}_{\mathrm{CF}}$ is the temperature corresponding to maxima (peaks) in the $\Delta \mathrm{CF}$ leakage/ $\Delta \mathrm{T}$ results. Dual peaks reflecting $\mathrm{T}_{\mathrm{p}}$ and $\mathrm{T}_{\mathrm{m}}$ are shown, where applicable, and the greater of the 2 peaks are boldfaced.

${ }^{\mathrm{d}} \Delta \mathrm{T}_{1 / 2}$ is the width of the main transition curve at half height of the peak.

ePretransition peak merged with melting transition peak. For $\mathrm{L}: \mathrm{N}=5,000: 1$ the pretransition was not observed.

DPPC liposomes exhibited maximum changes in CF leakage at temperatures corresponding to the $\left(\mathrm{L}_{\beta} \rightarrow \mathrm{P}_{\beta}{ }^{\prime}\right)$ pretransition (exothermic peak at $\left.35.7^{\circ} \mathrm{C}\right)$ and the $\left(\mathrm{P}_{\beta}{ }^{\prime}\right.$ $\rightarrow \mathrm{L}_{\alpha}$ ) melting transition (exothermic peak at $41.1^{\circ} \mathrm{C}$ ), (Figure 4-6). There is remarkable agreement between the peaks for $\Delta \mathrm{CF}$ leakage/ $\Delta \mathrm{T}$ and for $\mathrm{C}_{\mathrm{p}}$ associated with the pretransition and melting transition. When the pretransition peak emerged between $30-31{ }^{\circ} \mathrm{C}$, the large $\Delta \mathrm{CF}$ leakage/ $\Delta \mathrm{T}$ peak also emerged. When this peak 
plateaued between $37-38^{\circ} \mathrm{C}$ the $\Delta \mathrm{CF}$ leakage/ $\Delta \mathrm{T}$ peak also plateaued. This same trend was found for the melting transition. Gel phase lipids are ordered with fully extended acyl tails in the trans conformation, whereas fluid phase lipids are disordered in the gauche conformation and exhibit a thinner bilayer. ${ }^{33}$ The rippled gel phase, while not completely understood, is defined as a gel phase lipid bilayer with periodic domains of fluid phase lipids. The bilayer ripples are caused by the differences in gel and fluid phase bilayer thickness and hydration. ${ }^{36,37}$ Changes in CF leakage are greatest during these transitions because they represent co-existing phase domains within the bilayers where transient leakage is high at the interface between the domains. Our results show that the change in $\mathrm{CF}$ leakage is greatest during the pretransition where $\mathrm{L}_{\beta} \rightarrow \mathrm{P}_{\beta}{ }^{\prime}$ phases coexist. 

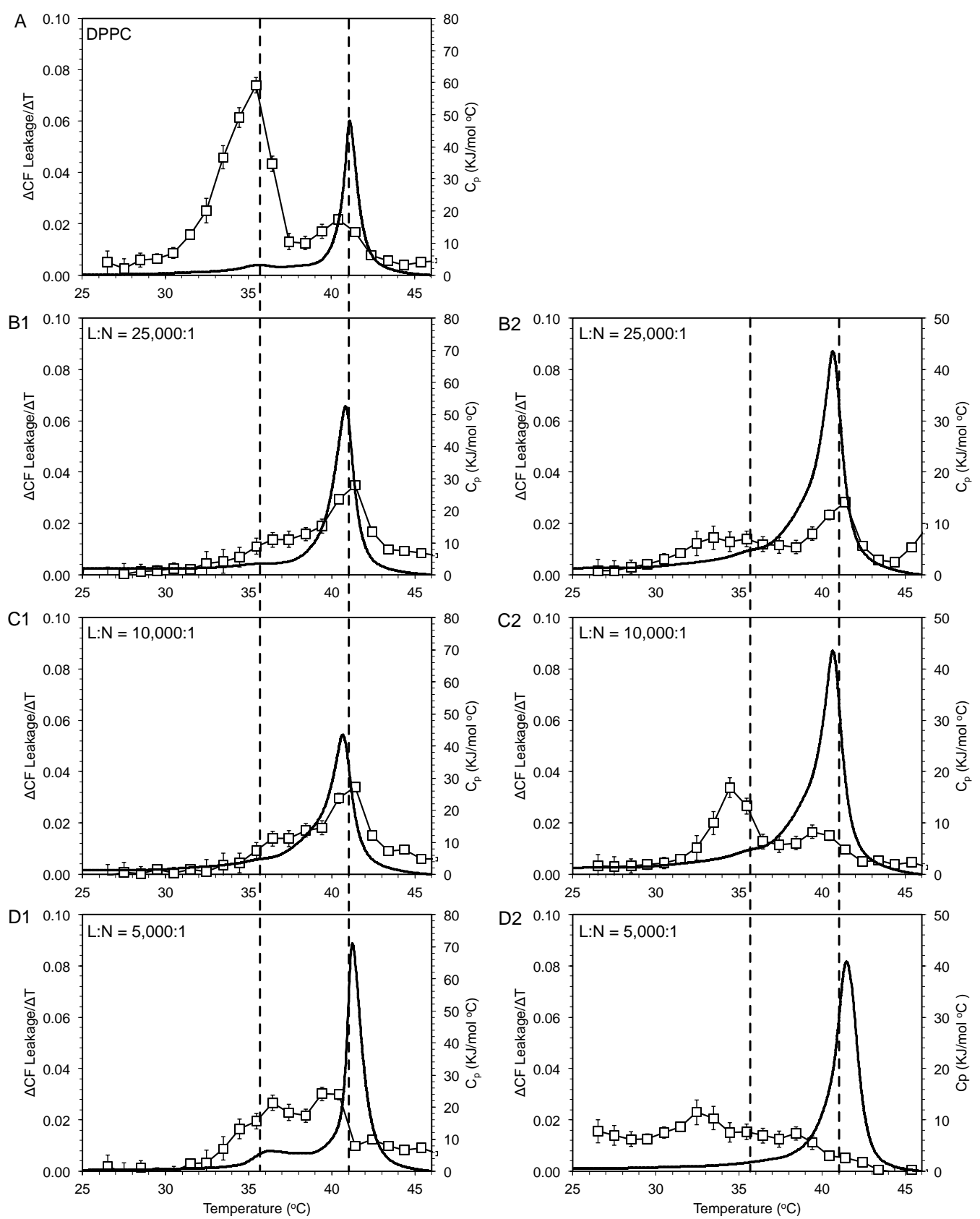

Figure 4-6. Change in CF leakage over change in time and excess heating capacity as a function of temperature.

Change in $C F$ leakage over the change in temperature $(\triangle C F$ leakage/ $\Delta T, \square)$ and excess heat capacity ( $\mathrm{C}_{\mathrm{p}}$, solid line, -) for DPPC (A), GNP2 DLNAs (B1, C1, and D1), and GNP4 DLNAs (B2, C2, and D2) at different lipid to nanoparticle ratios (L:N). For $\Delta C F$ leakage/ $\Delta T$, The vertical dashed lines correspond to the pretransition $\left(T_{p}\right)$ and melting ( $\left.\mathrm{T}_{\mathrm{m}}\right)$ temperatures for DPPC liposomes. For the $\Delta \mathrm{CF}$ leakage/ $\Delta \mathrm{T}$ graphs, the symbols and error bars correspond to the average \pm S.D. 
D-LNAs formed with GNP2 do not exhibit pretransition or melting temperatures that are significantly different than that of DPPC based on the position of the peaks (Figure 4-6B1, C1, and D1, Table 4-2). However, the changes in CF leakage measured for the D-LNAs are considerably different. For DPPC, the greatest change in $\mathrm{CF}$ leakage occurred during the pretransition. In comparison, the change in $\mathrm{CF}$ leakage for the D-LNAs corresponding to the pretransition was much smaller, and the change in CF leakage corresponding to melting was larger. The effects of GNP loading on the bilayer phase transitions and associated CF leakage is more pronounced when D-LNAs were prepared with GNP4, though the behavior is different than that for GNP2. In this case, changes in CF leakage are observed at temperatures corresponding to the pretransition despite the fact that increasing GNP4 loading reduced or eliminated this transition (Figure 6B2, C2, and D2). The change in CF leakage was shifted to lower temperatures and at the highest GNP4 loading (L:N = 5,000:1) significant CF leakage occurs between $26{ }^{\circ} \mathrm{C}$ and $38{ }^{\circ} \mathrm{C}$.

GNP4 loading also had a much larger effect on the interaction of bilayer lipids during main phase transition based on $\Delta \mathrm{T}_{1 / 2}$ (Table 4-2). $\Delta \mathrm{T}_{1 / 2}$ is the width of the main transition curve at half the height of the peak. This parameter relates to the cooperativity of neighboring lipids when undergoing the rippled gel to fluid phase transition ${ }^{38}$. For a first-order lipid phase transition, the peak theoretically should be infinitely sharp because all the lipids would undergo the phase transition as a single unit. Broadening of the main transition peak is caused by the occurrence of lipids in 
multiple melting domains and phase states ${ }^{39,40}$ For GNP2, only at L:N = 5,000:1 can a change in $\Delta \mathrm{T}_{1 / 2}$ be observed. Loading of GNP4 showed a $68-93 \%$ change in $\Delta \mathrm{T}_{1 / 2}$. Analyzing the CF leakage results in Figures 4-5 and 4-6 suggests that the GNPs influence the thermal release behavior (bilayer permeability) by hindering or altering the bilayer phase behavior. Cryo-TEM results further show that the GNPs alter the D-LNA size and shape, relative to DPPC liposomes, which would also influence the release behavior (i.e. CF leakage represents a mass flowrate after time $t$, which is proportional to permeability $\times$ surface area). This depicts an exciting yet complicated relationship between bilayer permeability and D-LNA structure, where GNP size and loading could be used to control the release behavior in a temperaturedependent way. What is most striking is that the changes in CF leakage and lipid phase behavior occurred at such low volume fractions of GNPs within the bilayer (Table 4-1). Multiple mechanisms could be at work including (i) changes in lipid phase behavior and stabilization or destabilization of lipid phase domains during phase transitions (inferred by this and previous work); ${ }^{8,15,20,41,42}$ (ii) changes in bilayer structure and mechanics such as thickness and elasticity, respectively; ${ }^{9,15-17,19}$; and (iii) changes in liposome size and structure, (also inferred by this and previous work) and the formation of non-bilayer structures. ${ }^{8}$ These mechanisms are interrelated and likely occur simultaneously, necessitating additional work to determine the how these mechanisms contribute to the release behavior.

Additional insight can be gained by considering molecules that partition into lipid bilayers and are known to influence lipid organization, which in turn influences inter-lipid interactions that drive phase transitions and the kinetics associated with 
these transitions. The characteristic time of a pretransition has been reported as $5 \pm 2$ min. ${ }^{43}$ With a DSC scan rate of $1{ }^{\circ} \mathrm{C} \min ^{-1}$, results for DPPC show that the pretransition peak present over a $3{ }^{\circ} \mathrm{C}$ temperature range is consistent with this characteristic time. In addition to GNPs disrupting lipid organization, either locally or globally within the bilayer, the GNPs would also exhibit low translational diffusion times within the bilayers that would impact the kinetics associated with lipid reorganization. This was apparent during the D-LNA pretransitions, where they were suppressed or merged with the melting transition at higher temperatures, and the greatest for GNP3-4 which occupy more space within the bilayer and are less mobile than GNP2. Given that CF leakage is greatest at the interface between phase domains, the net effect of inhibiting or delaying a phase transition would be lower CF leakage or an apparent temperature lag.

\section{Conclusions}

As shown for the GNP D-LNAs, the permeability and phase behavior of liposomes can be manipulated by the size and concentration of embedded nanoparticles at low volume fractions within the bilayers. A lipid bilayer is approximately 4-5 $\mathrm{nm}$ thick, and nanoparticles that were smaller than the bilayer thickness (GNP2) affected the bilayer differently than nanoparticles that were closer to the bilayer thickness (GNP4). GNP2 caused the pretransition and main transition to merge while maintaining similar phase transition temperatures observed for DPPC liposomes. Unlike DPPC liposomes, which exhibited the greatest leakage at the

pretransition temperature, the merger of pretransition and main transition caused by 
GNP2 corresponded to the merger of the thermal leakage curves associated with these transitions. These smaller nanoparticles demonstrated the ability to reduce spontaneous leakage at lower temperatures by providing greater bilayer stability. In contrast, GNP4 suppressed the pretransition while broadening the main transition, and maximum leakage was observed near the pretransition temperature or at lower temperatures. These findings demonstrate that liposome leakage and stability can be manipulated by the size of embedded nanoparticles even at low loadings. Thus, embedded nanoparticles can be used to engineer D-LNAs with desired release characteristics for therapeutic applications. The nanoparticles also influence the thermal leakage behavior, allowing temperature to be used as a triggered release mechanism. Understanding how nanoparticles effect the behavior of lipid bilayers is important to the development of new multifunctional drug delivery vehicles from lipid nanoparticle assemblies.

\section{Acknowledgements}

This material is based upon work supported by the National Science Foundation under grant numbers CBET-1055652 and CBET-1337061. We gratefully acknowledge Yanjing Chen and Richard Kingsley for their assistance with cryo-TEM, Everett Crisman for his assistance with FE-SEM, and Arijit Bose, Yanina Breakiron, and Fiaz Mohammed for their assistance, advice, and support on this research. DLS, FE-SEM, and TEM facilities were provided through the RI Consortium for Nanoscience and Nanotechnology. 


\section{References}

1. Preiss, M. R.; Bothun, G. D. Stimuli-responsive liposome-nanoparticle assemblies. Expert opinion on drug delivery 2011, 8, 1025-40.

2. Preiss, M. R.; Gupta, A.; Bothun, G. D. Liposome-Nanoparticle Assemblies. Bionanotechnology: Biological Self-assembly and Its Applications 2013, 273.

3. Al-Jamal, W. T.; Kostarelos, K. Liposome-nanoparticle hybrids for multimodal diagnostic and therapeutic applications. Nanomedicine 2007, 2, 85-98.

4. Allen, T. M.; Cullis, P. R. Liposomal drug delivery systems: from concept to clinical applications. Advanced drug delivery reviews 2013, 65, 36-48.

5. Chang, H. I.; Yeh, M. K. Clinical development of liposome-based drugs: formulation, characterization, and therapeutic efficacy. International journal of nanomedicine 2012, 7, 49-60.

6. Wi, H. S.; Lee, K.; Pak, H. K. Interfacial energy consideration in the organization of a quantum dot-lipid mixed system. Journal of Physics: Condensed Matter 2008, 20, 494211.

7. Ginzburg, V. V.; Balijepalli, S. Modeling the Thermodynamics of the Interaction of Nanoparticles with Cell Membranes. Nano Letters 2007, 7, 3716-3722.

8. Chen, Y.; Bose, A.; Bothun, G. D. Controlled release from bilayer-decorated magnetoliposomes via electromagnetic heating. ACS nano 2010, 4, 3215-21.

9. Amstad, E.; Kohlbrecher, J.; Müller, E.; Schweizer, T.; Textor, M.; Reimhult, E. Triggered Release from Liposomes through Magnetic Actuation of Iron Oxide Nanoparticle Containing Membranes. Nano Letters 2011, 11, 1664-1670. 
10. Gopalakrishnan, G.; Danelon, C.; Izewska, P.; Prummer, M.; Bolinger, P.-Y.; Geissbühler, I.; Demurtas, D.; Dubochet, J.; Vogel, H. Multifunctional Lipid/Quantum Dot Hybrid Nanocontainers for Controlled Targeting of Live Cells. Angewandte Chemie International Edition 2006, 45, 5478-5483.

11. Bothun, G. D.; Rabideau, A. E.; Stoner, M. A. Hepatoma Cell Uptake of Cationic Multifluorescent Quantum Dot Liposomes. The Journal of Physical Chemistry B 2009, 113, 7725-7728.

12. Al-Jamal, W. T.; Al-Jamal, K. T.; Bomans, P. H.; Frederik, P. M.; Kostarelos, K. Functionalized-Quantum-Dot-Liposome Hybrids as Multimodal Nanoparticles for Cancer. Small 2008, 4, 1406-1415.

13. Al-Jamal, W. T.; Al-Jamal, K. T.; Tian, B.; Lacerda, L.; Bomans, P. H.; Frederik, P. M.; Kostarelos, K. Lipid-Quantum Dot Bilayer Vesicles Enhance Tumor Cell Uptake and Retentionin Vitroandin Vivo. ACS nano 2008, 2, 408418.

14. Binder, W. H.; Sachsenhofer, R.; Farnik, D.; Blaas, D. Guiding the location of nanoparticles into vesicular structures: a morphological study. Physical chemistry chemical physics : PCCP 2007, 9, 6435-41.

15. Von White, G., 2nd; Chen, Y.; Roder-Hanna, J.; Bothun, G. D.; Kitchens, C. L. Structural and thermal analysis of lipid vesicles encapsulating hydrophobic gold nanoparticles. ACS nano 2012, 6, 4678-85.

16. Rasch, M. R.; Rossinyol, E.; Hueso, J. L.; Goodfellow, B. W.; Arbiol, J.; Korgel, B. A. Hydrophobic Gold Nanoparticle Self-Assembly with Phosphatidylcholine 
Lipid: Membrane-Loaded and Janus Vesicles. Nano Letters 2010, 10, 37333739.

17. Park, S. H.; Oh, S. G.; Mun, J. Y.; Han, S. S. Loading of gold nanoparticles inside the DPPC bilayers of liposome and their effects on membrane fluidities. Colloids and surfaces. B, Biointerfaces 2006, 48, 112-8.

18. Paasonen, L.; Sipila, T.; Subrizi, A.; Laurinmaki, P.; Butcher, S. J.; Rappolt, M.; Yaghmur, A.; Urtti, A.; Yliperttula, M. Gold-embedded photosensitive liposomes for drug delivery: triggering mechanism and intracellular release. Journal of controlled release : official journal of the Controlled Release Society $2010,147,136-43$.

19. Park, S. H.; Oh, S. G.; Mun, J. Y.; Han, S. S. Effects of silver nanoparticles on the fluidity of bilayer in phospholipid liposome. Colloids and surfaces. B, Biointerfaces 2005, 44, 117-22.

20. Bothun, G. D. Hydrophobic silver nanoparticles trapped in lipid bilayers: Size distribution, bilayer phase behavior, and optical properties. Journal of nanobiotechnology 2008, 6, 13.

21. Jang, H.; Pell, L. E.; Korgel, B. A.; English, D. S. Photoluminescence quenching of silicon nanoparticles in phospholipid vesicle bilayers. Journal of Photochemistry and Photobiology A: Chemistry 2003, 158, 111-117.

22. Chen, Y.; Bothun, G. D. Lipid-assisted formation and dispersion of aqueous and bilayer-embedded nano-C60. Langmuir : the ACS journal of surfaces and colloids 2009, 25, 4875-9. 
23. Jeng, U. S.; Hsu, C.-H.; Lin, T.-L.; Wu, C.-M.; Chen, H.-L.; Tai, L.-A.; Hwang, K.-C. Dispersion of fullerenes in phospholipid bilayers and the subsequent phase changes in the host bilayers. Physica B: Condensed Matter 2005, 357, 193-198.

24. Santhosh, P. B.; Penič, S.; Genova, J.; Iglič, A.; Kralj-Iglič, V.; Ulrih, N. P. A study on the interaction of nanoparticles with lipid membranes and their influence on membrane fluidity. Journal of Physics: Conference Series 2012, $398,012034$.

25. Bonnaud, C.; Monnier, C. A.; Demurtas, D.; Jud, C.; Vanhecke, D.; Montet, X.; Hovius, R.; Lattuada, M.; Rothen-Rutishauser, B.; Petri-Fink, A. Insertion of Nanoparticle Clusters into Vesicle Bilayers. ACS nano 2014, 8, 3451-3460.

26. Rasch, M. R.; Yu, Y.; Bosoy, C.; Goodfellow, B. W.; Korgel, B. A. ChloroformEnhanced Incorporation of Hydrophobic Gold Nanocrystals into Dioleoylphosphatidylcholine (DOPC) Vesicle Membranes. Langmuir : the ACS journal of surfaces and colloids 2012, 28, 12971-12981.

27. Lee, H.-Y.; Shin, S. H. R.; Abezgauz, L. L.; Lewis, S. A.; Chirsan, A. M.; Danino, D. D.; Bishop, K. J. M. Integration of Gold Nanoparticles into Bilayer Structures via Adaptive Surface Chemistry. Journal of the American Chemical Society 2013, 135, 5950-5953.

28. Rasch, M. R.; Bosoy, C. A.; Yu, Y.; Korgel, B. A. Chains, Sheets, and Droplets: Assemblies of Hydrophobic Gold Nanocrystals with Saturated Phosphatidylcholine Lipid and Squalene. Langmuir : the ACS journal of surfaces and colloids 2012, 28, 15160-15167. 
29. Brust, M.; Walker, M.; Bethell, D.; Schiffrin, D. J.; Whyman, R. Synthesis of thiol-derivatised gold nanoparticles in a two-phase Liquid?Liquid system. Journal of the Chemical Society, Chemical Communications 1994, 801.

30. Torchilin, V. P.; Weissig, V. Liposomes : a practical approach. 2nd ed.; Oxford University Press: Oxford ; New York, 2003; p xxiii, 396 p.

31. Abramoff, M.; Magalhaes, P.; Ram, S. Image Processing with ImageJ. Biophotonics International 2004, 36-42.

32. Park, Y.-K.; Park, S. Directing Close-Packing of Midnanosized Gold Nanoparticles at a Water/Hexane Interface. Chemistry of Materials 2008, 20, 2388-2393.

33. Nagle, J. F.; Tristram-Nagle, S. Structure of lipid bilayers. Biochimica et Biophysica Acta (BBA) - Reviews on Biomembranes 2000, 1469, 159-195.

34. Koynova, R.; Caffrey, M. Phases and phase transitions of the phosphatidylcholines. Biochimica et biophysica acta 1998, 1376, 91-145.

35. Hashizaki, K.; Taguchi, H.; Sakai, H.; Abe, M.; Saito, Y.; Ogawa, N. Carboxyfluorescein leakage from poly (ethylene glycol)-grafted liposomes induced by the interaction with serum. Chemical and pharmaceutical bulletin 2006, 54, 80-84.

36. Heimburg, T. A model for the lipid pretransition: coupling of ripple formation with the chain-melting transition. Biophysical journal 2000, 78, 1154-65.

37. Tristram-Nagle, S.; Nagle, J. F. Lipid bilayers: thermodynamics, structure, fluctuations, and interactions. Chemistry and physics of lipids 2004, 127, 3-14. 
38. Benesch, M. G.; Mannock, D. A.; Lewis, R. N.; McElhaney, R. N. A calorimetric and spectroscopic comparison of the effects of lathosterol and cholesterol on the thermotropic phase behavior and organization of dipalmitoylphosphatidylcholine bilayer membranes. Biochemistry 2011, 50, 9982-97.

39. Stillwell, W. Chapter 9 - Basic Membrane Properties of the Fluid Mosaic Model. In An Introduction to Biological Membranes, Elsevier: San Diego, 2013; pp 131174.

40. Demetzos, C. Differential Scanning Calorimetry (DSC): a tool to study the thermal behavior of lipid bilayers and liposomal stability. Journal of liposome research 2008, 18, 159-73.

41. Qiu, D.; An, X. Q.; Chen, Z. Y.; Ma, X. Y. Microstructure study of liposomes decorated by hydrophobic magnetic nanoparticles. Chemistry and physics of lipids 2012, 165, 563-570.

42. Lin, X.; Gu, N. Surface properties of encapsulating hydrophobic nanoparticles regulate the main phase transition temperature of lipid bilayers: A simulation study. Nano Research 2014, 7, 1195-1204.

43. Lentz, B. R.; Freire, E.; Biltonen, R. L. Fluorescence and calorimetric studies of phase transitions in phosphatidylcholine multilayers: kinetics of the pretransition. Biochemistry 1978, 17, 4475-4480. 


\title{
CHAPTER 5
}

\section{MRI Relaxivity and siRNA Binding Capacity of Lipid-Coated Magnetic} Nanoparticles Controlled by Polyethylene Glycol Confirmation

\author{
Matthew Ryan Preiss ${ }^{1}$, Eily Cournoyer ${ }^{1}$, Kayla Belanger ${ }^{1}$, Edward Walsh ${ }^{2}$, \\ Niall Howlett ${ }^{3}$, and Geoffrey Bothun ${ }^{1 *}$ \\ In Preparation for: Nano Letters
}

${ }^{1}$ Department of Chemical Engineering, University of Rhode Island, 16 Greenhouse Road, Kingston, RI 02881

${ }^{2}$ Department of Neuroscience, Department of Diagnostic Imaging, Institute for Brain Science, Institute for Molecular and Nanoscale Innovation, Associate Director for MRI Physics, Brown University, Sidney E. Frank Hall, 185 Meeting Street, Providence, RI 02912

${ }^{3}$ Department of Cell and Molecular Biology, University of Rhode Island, 379 CBLS Building, Kingston, RI 02881

${ }^{\dagger}$ Author for correspondence: Geoffrey D Bothun

Department of Chemical Engineering University of Rhode Island 205 Crawford Hall, 16 Greenhouse Road, Kingston, RI, 02881, USA

Phone: +1-401-874-9518

Email: gbothun@uri.edu 


\section{Abstract}

The development of multifunctional bionanotheranostic devices are the pinnacle of biomedical research and represent a major step towards the development of personalized medicine. Here, lipid coated magnetic nanoparticles (LMNPs) were created by coating iron oxide nanoparticles with a PEG (2000 MW) and cationic lipid using a dual solvent exchange (DSE) method. LMNPs were created with 0-100 mol\% PEG and their structure, zeta potential $(\zeta)$, MRI $r_{2}$ relaxivity, binding and release siRNA, and heating when subjected to an electromagnetic field (EMF). Based on size and $\zeta$, it was confirmed that the DSE method could control the surface composition of lipids. PEG surface density and conformation was shown to have an effect on the characteristics of LMNPs. MRI $r_{2}$ relaxivity could be controlled by the surface composition of PEG. The greater the PEG surface composition the greater the $\mathrm{r}_{2}$ relaxivity. LMNPs were also shown to be able to bind siRNA. Release of siRNA could be triggered by temperature or when subjected to EMF. The LMNPs developed are bionanotheranostic devices combining the enhancement of MRI $\mathrm{r}_{2}$ relaxivities, triggered siRNA release, and hyperthermia. 
Bionanotheranostics (BNT) involves the application of biology and nanotechnology to develop platforms with theranostic (dual therapeutic and diagnostic functions) capabilities. Superparamagnetic iron oxide nanoparticles (SPIONSs) are well established for biomedical applications and provide an attractive platform for designing such multifunctional BNTs. The magnetic properties of SPIONs allow them to be used as an MRI contrast enhancement agent and for magnetic targeting. Also, SPIONs generate heat when subjected to an external alternating magnetic field, which can be used for hyperthermia and for triggered release from the surface. ${ }^{1}$ In addition to providing colloidal stability, minimizing clearance in vivo, and achieving cellular targeting, the ability to control the surface composition and chemistry can impart the SPION with additional capabilities, such as drug and oligonucleotide delivery where these molecules can be incorporated within or adsorbed to the coating. ${ }^{1}$

Polyethylene glycol (PEG) is a hydrophilic biocompatible polymer commonly used to improve the blood circulation half-life, surface hydrophilicity, and reduce cytotoxicity of nanoparticles. ${ }^{2-5}$ PEG is the most heavily studied surface modifying polymer for improving the efficiency and effectiveness of therapeutics. PEG has the ability to make a particle "stealth", improving its pharmacokinetics by reducing mononuclear phagocyte (immune) system uptake. ${ }^{6}$ However, there is no consensus as to what the optimum PEG coverage-density, conformation, and molecular weight to prevent necessary to prevent uptake by the immune system., ${ }^{3,7}$ PEG has been used for numerous clinical applications, including drug delivery, therapeutic targeting, reducing toxicity, preventing uptake by the immune system, and improving circulation in the blood by preventing clearance. PEG has also been used to in conjunction with 
SPIONs for the development BNTs. PEG and SPIONs have been particularly interesting in the development of diagnostics, demonstrating the ability to enhance the MRI signals. SPION MRI signal is heavily influenced by both the thickness and the chemical composition of the SPION surface coating and its effect on the diffusivity of water. The SPION magnetic moment influence the magnetic dipole relaxation of water held in close proximity to the SPION surface by hydrophilic coatings, such as PEG. Relaxivity increases because PEG influences neighboring water molecules by surface exclusion, hindering diffusion, and hydrogen bond immobilization. ${ }^{9,10}$ Therefore, functionalizing with polyethylene glycol (PEG) is an obvious route to achieve the hydration shell needed for negative MRI contrast agent. Nanoparticle PEGylation has also been shown to inhibit protein adsorption and recognition by the immune system, improving the pharmacokinetics and cytotoxicity of nanoparticles..$^{3-5}$ This work provides insight into selecting PEGylation strategies to optimize negative contrast for MRI imaging.

This research extends the concept of surface coating engineering of SPIONs to lipid-coated magnetic nanoparticles (LMNPs) with different compositions of cationic and PEG-lipids designed for combined MRI contrast enhancement, hyperthermia, and delivery of small interfering ribonucleic acid (siRNA). Cationic DOTAP (1,2dioleoyl-3-trimethylammonium-propane) lipid electrostatically interacts with the negatively charged phosphate backbone of nucleic acids, enabling siRNA to be bound to the surface of the LMNPs. ${ }^{5,11-13}$ LMNPs were formed with surface lipids compositions of methoxy-terminated $\mathrm{PEG}_{2000}$ anchored to a DMPE lipid (1,2dimyristoyl-sn-glycero-3-phosphoethanolamine-N-[methoxy(polyethylene glycol)] 
(2000 MW)) and the balance of the lipid surface composition comprised of DOTAP. Hereinafter, LMNP samples will be identified by the surface lipid mol\% of PEG2000. We systematically examined the effect of the surface PEG concentration on physicochemical properties of LMNPs, the transverse magnetic relaxivity $\left(\mathrm{r}_{2}\right)$, and the siRNA binding capacity. These properties are directly related to PEG-lipid concentration and PEG conformation on the LMNP surface. Furthermore, we present results demonstrating that PEG conformation also influences the release of siRNA due to temperature or in the presence of an alternating electromagnetic field where heat is provided by the SPIONs.

Monodispersed LMNPs were formed using a dual solvent exchange (DSE) method to control the lipid surface composition. ${ }^{14}$ DSE induces the adsorption of lipids onto the hydrophobic SPION surface by changing the polarity of the suspending solvents rendering water soluble LMNPs. LMNPs were formed by coating hydrophobic (oleic acid-coated) SPIONs with cationic DOTAP and/or PEG-DMPE (DMPE modified with a PEG-terminated headgroup). DSE involves initially mixing SPIONs and lipids at the desired composition in chloroform. In the first solvent exchange, chloroform is replaced with dimethyl sulfoxide (DMSO), a solvent miscible in both chloroform and water, by rotary evaporation. This exchange induces the hydrophobic tail of DOTAP and PEG-DMPE lipids to assemble on the hydrophobic SPION surface. The second solvent exchange involves replacing DMSO with water by ultracentrifugation, further adhering the lipids to the SPION surface, rendering water soluble LMNPs. ${ }^{14}$ 


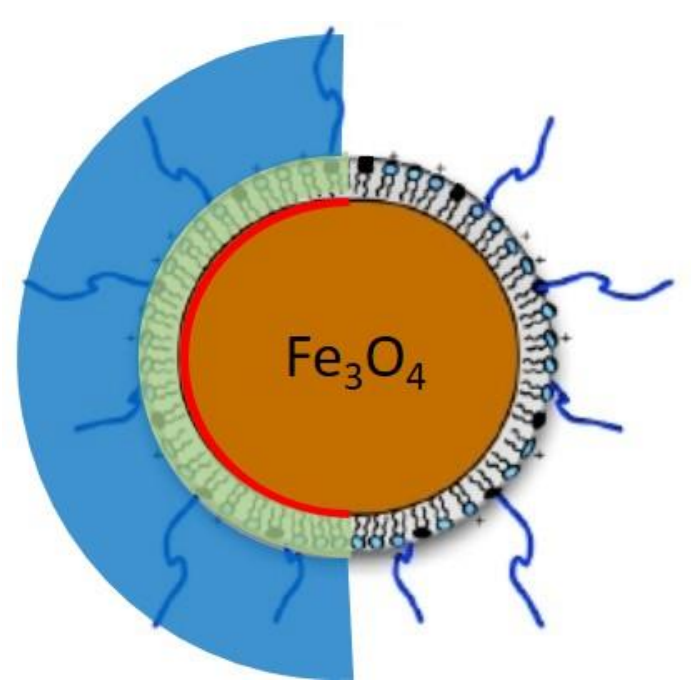

\section{Figure 5-1. Schematic of LMNP.}

LMNP consisting of an iron oxide core, a hydrophobic oleic acid coating (red), a lipid monolayer (green), and PEG layer (blue).

LMNPs are comprised of 4 layers: (1) iron oxide core, (2) hydrophobic oleic acid coating, (3) lipid monolayer, and (4) PEG layer (Figure 5-1). A SPION core is coated with oleic acid making it hydrophobic. A lipid monolayer forms on the surface by hydrophobic interaction between the lipid tails of DOTAP and PEG-DMPE and oleic acid. The hydrophilic DOTAP and PEG-DMPE lipid heads faces away from the surface making the LMNPs water soluble. Finally, PEG chains attached to the DMPE lipid head extend away from the monolayer forming a water permeable layer. 


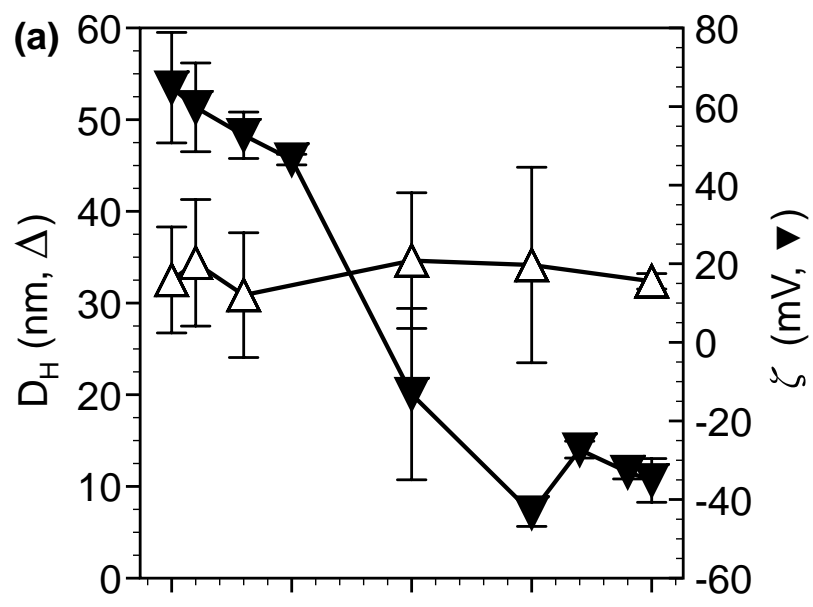

(c)
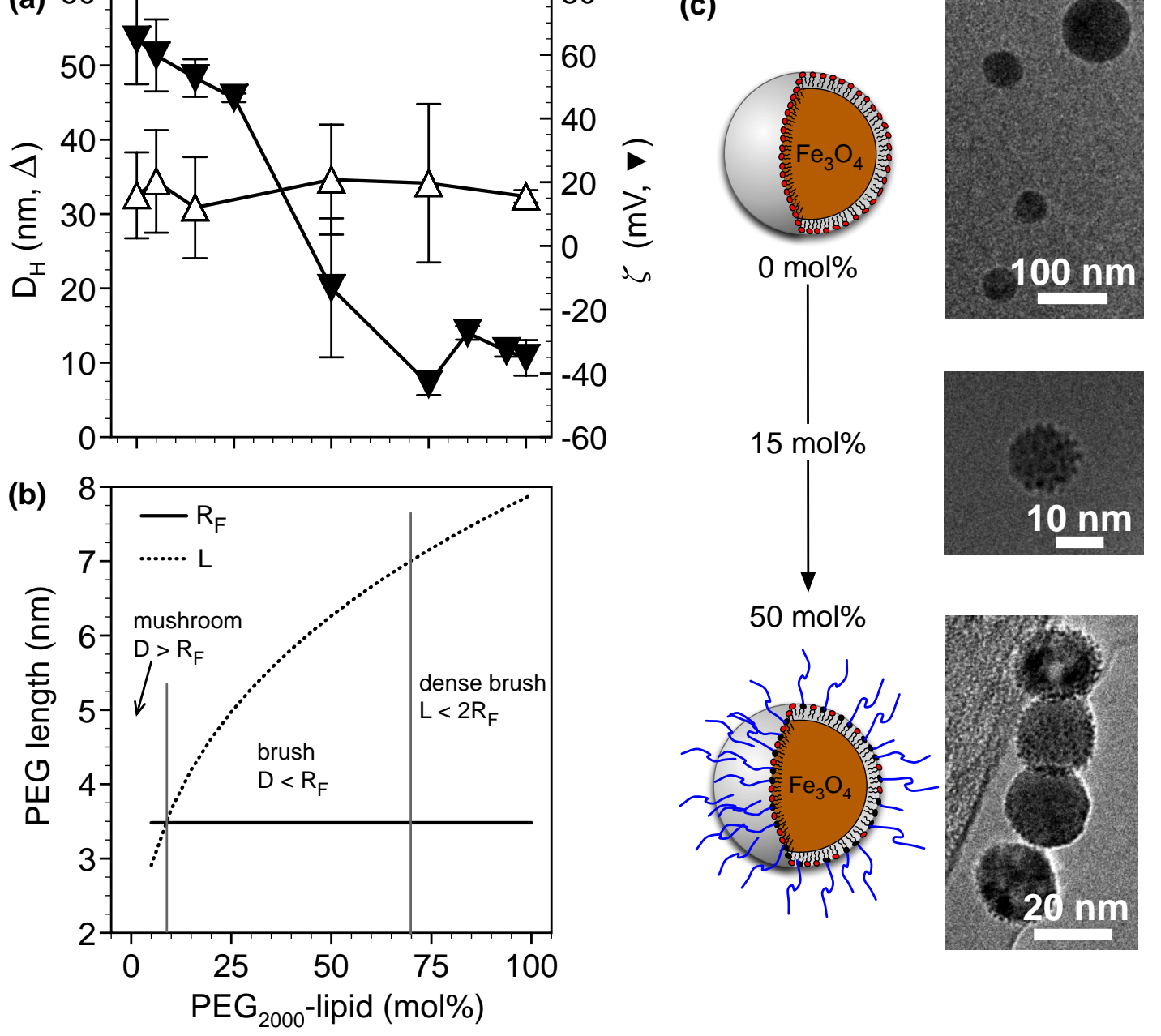

Figure 5-2. (a) LMNP Measured Hydrodynamic Diameter and $\zeta$, (b) Predicted PEG Length and Polymer Conformation, (c) Schematic and cryogenic transmission electron microscope images of LMNPs.

(a) Hydrodynamic diameter and $\zeta$ versus LMNP surface mol\% of PEG2000 (average \pm S.D.) (b) Theoretical Flory radius, $\mathrm{PEG}_{2000}$ length, and $\mathrm{PEG}_{2000}$ conformation as a function LMNP surface mol\% of $\mathrm{PEG}_{2000}$. (c) Schematic and cryogenic transmission electron microscope (Cryo-TEM) images of LMNPs with 0, 15, and $50 \mathrm{~mol} \% \mathrm{PEG}_{2000}$ surface concentration. 
Table 5-1 Parameters used for calculation of Flory radius, PEG footprint, distance between PEG groups, area occupied by PEG, PEG chain length, and LMNP diameter.

\begin{tabular}{|c|c|c|c|c|}
\hline Parameter & Definition & Value & Units & Ref. \\
\hline \multicolumn{5}{|c|}{ Flory Radius } \\
\hline $\mathrm{a}$ & PEG monomer length & 0.35 & $\mathrm{~nm}$ & \\
\hline $\mathrm{N}$ & $\begin{array}{l}\text { Number of repeat monomer } \\
\text { units for } \mathrm{PEG}_{2000}\end{array}$ & 44 & & \\
\hline $\mathrm{R}_{\mathrm{F}}$ & Flory radius & 3.4 & $\mathrm{~nm}$ & \\
\hline \multicolumn{5}{|c|}{$P E G_{2000}$} \\
\hline FP & $\begin{array}{l}\text { Footprint or projected area of a } \\
\text { single PEG chain on the LMNP } \\
\text { surface }\end{array}$ & 36.1 & $\begin{array}{l}\mathrm{nm}^{2} \text { per } \\
\text { PEG }\end{array}$ & \\
\hline \multicolumn{5}{|c|}{ Iron Oxide Nanoparticles } \\
\hline DSPION & Diameter of SPION & $28.3 \pm 6.8$ & $\mathrm{~nm}$ & \\
\hline SSPION & \begin{tabular}{|l|}
$\begin{array}{l}\text { Surface area of iron oxide } \\
\text { nanoparticle }\end{array}$ \\
\end{tabular} & 2516 & $\mathrm{~nm}^{2}$ & \\
\hline \multicolumn{5}{|c|}{ Lipids } \\
\hline $\mathrm{T}_{\mathrm{B}, \mathrm{DOTAP}}$ & DOTAP bilayer thickness & 4.2 & $\mathrm{~nm}$ & \\
\hline $\mathrm{T}_{\mathrm{B}, \mathrm{DMPE}}$ & DMPE bilayer thickness & 3.6 & $\mathrm{~nm}$ & \\
\hline ADOTAP & Cross-sectional area of DOTAP & 0.53 & $\mathrm{~nm}^{2}$ & \\
\hline AdMPE & Cross-sectional area of DMPE & 0.52 & $\mathrm{~nm}^{2}$ & \\
\hline
\end{tabular}

The effect of the surface composition of PEG on the LMNPs was assessed by measuring the hydrodynamic diameter, $\mathrm{D}_{\mathrm{H}}$, and zeta-potential, $\zeta$, using dynamic light scattering (DLS). The $\mathrm{D}_{\mathrm{H}}$ of the SPION core in chloroform was measured to be $28.3 \pm$ $6.8 \mathrm{~nm}$. The number-weighted mean $\mathrm{DH}$ as a function of mol\% PEG are shown in Figure 5-2(a). Number-weighted mean size from DLS was recorded because for surface-modified PEG nanostructures it is the recommended over z-average. For these 
structures, values calculated based on number distribution have been shown to be more comparable to other sizing techniques, specifically TEM. ${ }^{3}$

When PEG lipids are added, the length of PEG (and the LMNP's size) is determined by the structural conformation of the PEG polymer chain on the nanoparticle surface. PEG most commonly adopts the mushroom, brush, or dense brush conformations on a nanoparticle surface at a liquid/solid interface in a good solvent. However, if the PEG chain can interact with the nanoparticle surface, it can take on rarer conformations, such as pancake or loop (discussed below).

PEG's conformation is determined can be determined by 3 parameters: (1) the Flory radius which is the mean radius of the hemispherical volume occupied by an unperturbed random coil PEG polymer chain $\left(\mathrm{R}_{\mathrm{F}}\right),(2)$ the distance between PEG chains (D), and (3) the PEG chain length $(\mathrm{L})^{2,3}$. The parameters used for these calculations art in Table 5-1. The Flory radius, $\mathrm{R}_{\mathrm{F}}$, is calculated as:

$$
R_{F}=a N^{3 / 5}=3.4 n m
$$

where $a$ is the PEG monomer length and $\mathrm{N}$ is the number of repeat monomer units (parameters for calculations are in Table 5-1). ${ }^{3}$ The mean distance between PEG groups, D, is calculated with the following equation:

$$
D=2 \sqrt{\frac{A_{P E G}}{\pi}}
$$


where APEg is the area that a PEG chain occupies. APEg is calculated with the following:

$$
A_{P E G}=\frac{S_{N P}}{N_{P E G}}
$$

where $\mathrm{S}_{\mathrm{NP}}$ is the surface area of the iron oxide nanoparticle $\left(2516 \mathrm{~nm}^{2}\right)$ and $\mathrm{N}_{\mathrm{PEG}}$ is the number of PEG on each nanoparticle. NPEG is calculated based upon the mol\% PEG2000 and the cross-sectional area of the acyl lipid tails, ALIPID, of DOTAP and DMPE (the PEG anchor lipid). ${ }^{3}$

PEG takes on the mushroom conformation at low PEG density, $\mathrm{D}>\mathrm{R}_{\mathrm{F}}$ and $\mathrm{R}_{\mathrm{F}}$ $\geq \mathrm{L}^{2,}{ }^{15}$ In the mushroom regime, PEG chains have a random coil conformation with no interaction with adjacent PEG chains. When $\mathrm{D} \approx \mathrm{R}_{\mathrm{F}}$, individual PEG chains begin to take on the brush conformation. The brush conformation occurs when PEG chains are close enough to have some interaction that disrupting the ability of PEG chains to retain their mushroom conformation. ${ }^{15} \mathrm{PEG}$ is in the brush conformation when $\mathrm{R}_{\mathrm{F}}<\mathrm{D}$ $<2 \mathrm{R}_{\mathrm{F}}$. The dense brush conformation occurs at very high PEG densities when there is an increased amount of inter-chain interactions and steric repulsion forcing the PEG chains further away from the nanoparticle surface. The dense brush conformation occurs when $\mathrm{D}<\mathrm{RF}_{\mathrm{F}}$ and $\mathrm{L}<2 \mathrm{R}_{\mathrm{F}},{ }^{3}{ }^{15} \mathrm{PEG}_{2000}$ should be in the mushroom conformation below 9 mol\% PEG, in the brush conformation from 9 to 70 mol\% PEG, and in the dense brush conformation at greater than 70 mol\% PEG (Figure 5-2(b)) 
Table 5-2. Calculated PEG length, PEG footprint, and Calculated and Measured DLMNP.

\begin{tabular}{|c|c|c|c|c|}
\hline \multirow{2}{*}{ mol\% PEG } & \multirow{2}{*}{$\mathbf{L}(\mathbf{n m})$} & \multirow{2}{*}{ FP $\left.\mathbf{( n m}^{\mathbf{2}}\right)$} & \multicolumn{2}{|c|}{ D $_{\text {LMNP }}(\mathbf{n m})$} \\
\cline { 4 - 5 } & & & Calculated & Measured \\
\hline 0 & 0 & - & 32.5 & $31.7 \pm 12.0$ \\
\hline 5 & 3.4 & 36.1 & 38.7 & $29.9 \pm 11.1$ \\
\hline 15 & 4.3 & 57.6 & 40.5 & $36.8 \pm 10.8$ \\
\hline 25 & 5.1 & 81.1 & 42.1 & $41.2 \pm 12.0$ \\
\hline 50 & 6.4 & 129.1 & 44.7 & $39.2 \pm 12.0$ \\
\hline 75 & 7.3 & 169.7 & 46.6 & $37.3 \pm 11.0$ \\
\hline 100 & 8.1 & 206.2 & 48.1 & $39.1 \pm 10.8$ \\
\hline
\end{tabular}

The size of the LMNPs was calculated with the following equation:

$$
D_{L M N P}=2 L+D_{N P}+T_{B}
$$

where $\mathrm{L}$ is the length of the PEG chain, $\mathrm{DNP}_{\mathrm{NP}}$ is the diameter of the iron oxide nanoparticle core $(28.3 \pm 6.8 \mathrm{~nm})$, and $\mathrm{T}_{\mathrm{B}}$ is the thickness of the lipid bilayer. $\mathrm{T}_{\mathrm{B}}$ for DOTAP and DMPE (the anchor lipid for PEG) is $4.2 \mathrm{~nm}^{16}$ and $3.6 \mathrm{~nm}^{17}$, respectively. The calculated L, FP, and DLMNP and the measured DLMNP are recorded in Table 5-2.

At 0 mol\% PEG, the LMNPs are completely covered with DOTAP. Therefore, $\mathrm{L}=0 \mathrm{~nm}$ and $\mathrm{T}_{\mathrm{B}}$ was the thickness of DOTAP. The calculated and measured DLMNP were $32.5 \mathrm{~nm}$ and $31.7 \pm 12 \mathrm{~nm}$, respectively.

PEG2000 should take on the mushroom conformation below 9 mol\% PEG. When $\mathrm{PEG}_{2000}$ is in the mushroom conformation, $\mathrm{L}_{\text {Mushroom }}=\mathrm{R}_{\mathrm{F}}=3.4 \mathrm{~nm} \cdot{ }^{3} \mathrm{PEG}_{2000}$ should take on the mushroom conformation below 9 mol\% PEG. Only the 5 mol\% PEG LMNP sample should be in the mushroom conformation. The calculated $\mathrm{D}_{\mathrm{LMNP}}=$ $38.7 \mathrm{~nm}$. The calculated size does fall within the standard deviation of the measured size at $D_{\mathrm{H}} 29.9 \pm 11.1 \mathrm{~nm}$. 
The measured size may be smaller than expected because the PEG chains are methoxy-terminated giving them an anionic charge. When polymer chains are not repelled from the nanoparticle surface, a special "pancake" conformation has been observed. ${ }^{18}$ PEG lies along its own length on the nanoparticle surface. The PEG length in the pancake conformation, $\mathrm{L}_{\text {pancake, }}$ is calculated as:

$$
L_{\text {pancake }}=\frac{1}{\sqrt{N}}
$$

where, $\mathrm{N}$ is the number of repeat monomer units (44). ${ }^{3,18,19}$ For $\mathrm{PEG}_{2000}, \mathrm{~L}_{\text {pancake }}$ is 0.2 nm. The PEG 2000 lipid anchor is DMPE which has a bilayer thickness of $3.6 \mathrm{~nm} .{ }^{17}$ Therefore, the calculated size $5 \mathrm{~mol} \%$ PEG LMNPs is $32.2 \mathrm{~nm}$, which is close to the measured $\mathrm{D}_{\mathrm{H}}$ and well within the standard deviation. However, if the PEG chain is lying on the nanoparticle surface, the chain may be forcing other lipids to lie parallel to the surface. We do not have a way to account for this but it may be the cause of the smaller than calculated LMNPs.

PEG can also form a loop conformation when the unattached end of the polymer chain can absorb to the surface. The methoxy-terminated PEG lipid used here provides an anionic charge at the end of the PEG chain that could potentially attach to the cationic DOTAP surface forming a loop conformation. However, little research has been done on the behavior and characteristics of this conformation and no predictive equations to calculate LPEG were available. Despite, this could account for the overestimation of DLMNP. 
PEG chains in the brush conformation should start to appear on LMNPs with greater than 9 mol\% PEG. Brush layer thickness, Lbrush, is calculated as:

$$
L_{\text {brush }}=\frac{N a^{5 / 3}}{D^{2 / 3}}
$$

At $15 \mathrm{~mol} \%$ PEG, Lbrush $=4.3 \mathrm{~nm}$ and $\mathrm{D}_{\mathrm{H}}=40.5 \mathrm{~nm}$. Like $5 \mathrm{~mol} \% \mathrm{PEG}$, the predicted $\mathrm{D}_{\mathrm{H}}$ is larger than the measured $\mathrm{D}_{\mathrm{H}}=36.8 \pm 10.8 \mathrm{~nm}$, but well within the standard deviation. The measured $\mathrm{D}_{\mathrm{H}}$ then plateaus for all samples $\geq 25 \mathrm{~mol} \% \mathrm{PEG}$ around an average of $39.2 \mathrm{~nm}$, through the brush (9-70 mol\% PEG) and the dense brush (>70 mol\% PEG) conformations. The size plateau that spans both the brush and dense brush conformations is likely due to the structural similarities in the brush and dense brush regimes.

In the predicted brush regime, 25-50 mol\% PEG have calculated $D_{H}$ of 42.1 $\mathrm{nm}$ and $44.7 \mathrm{~nm}$, respectively, and measured $\mathrm{D}_{\mathrm{H}}$ are $41.2 \pm 12.0 \mathrm{~nm}$ and $39.2 \pm 12.0$ nm. In the dense brush regime, 75, and 100 mol\% PEG LMNPs have calculated $D_{H}$ of $46.6 \mathrm{~nm}$, and $48.1 \mathrm{~nm}$, respectively, and the measured $\mathrm{D}_{\mathrm{H}}$ are $37.3 \pm 11.0$, and $39.1 \pm$ 10.8, respectively. The calculated PEG length and LMNP diameter consistently overestimated the measured length and LMNP diameter. Despite, the calculated parameters were relatively accurate being within the measured standard deviation for all samples.

The size of the LMNP was approximately constant from 25-100 mol\% PEG. This size plateau is likely due to the anionic methoxy-terminated PEG forcing it towards the LMNP surface and restricting the PEG chain from extending away from the cationic surface charge. The lack of size change is unlikely to be caused by the 
LMNP surface being saturated with PEG because the $\zeta$ continues to change. The changing $\zeta$ suggests that the PEG concentration does not remain constant. Also, the size changes until the PEG conformation transitions from mushroom-to-brush suggesting that this behavior is related to the conformation and/or structure of the LMNP surface.

The overestimation of $\mathrm{D}_{\mathrm{H}}$ is likely due to the fact that the assumptions under which this system relies does not perfectly match with the LMNP system. The system used to calculate LMNP size assumes that the PEG chains are fixed to the lipid head of the PEG lipid, the lipids form a rigid fixed monolayer, the lipids extend away from the nanoparticle surface at full height, and the PEG chain does not interact with the nanoparticle surface, the PEG does not interdigitate into the LMNP lipid monolayer, and the PEG chains do not interact other than being sterically repelled. The accuracy of the above calculations relies most heavily on determining PEG surface coverage, which is affected by the above assumptions. PEG surface coverage, whether calculated as APEG, measured as the surface chain coverage PEG/nm $(\sigma)$, determined from the PEG footprint (FP), or by other means, defines the conformation of surface PEG and therein how it occupies the surface. The overestimation of $\mathrm{L}$ is likely due to the assumption that PEG chains remain fixed and their surface anchor point cannot move with the PEG chain. Despite, the calculated sizes were all within the measured standard deviation and all the calculations were greater than the measured size.

Also, the calculation of APEG, and therefore L, are based on the assumption that the DSE method coats the LMNPs with the lipid ratio as designed. While the $\zeta$ results and of other researchers with this method ${ }^{9,14,20}$, appear to validate this assumption, 
the DSE method has not been used for coating with lipids having significantly different structures. For example, Tong et. al. ${ }^{9}$ only tested the ability to control the surface ratios of DSPE-PEG 2000 with different functional groups $\left(\mathrm{NH}_{2}, \mathrm{COOH}\right.$, maleimide, and non-functionalized). It remains unclear how effectively the DSE method can control the surface composition of significantly different structures, such as DOTAP and DMPE-mPEG 2000 that have significantly different characteristics including critical micelle concentration and the presence of a linked polymer chain. The issue is further complicated by the ability of $\mathrm{PEG}_{2000}$ to screen the cationic DOTAP lipids in close proximity to the nanoparticle surface potentially skewing the $\zeta$ results. Despite these issues, the purpose of choosing PEG was to see if it could protect siRNA attached to the DOTAP close to the nanoparticle surface, similar to PEG's ability to protect structures from the immune system, and further functionalize iron oxide nanoparticles for multifunctional bionanotheranostics. The overestimation of LMNP size was likely due to an assumption that caused there to be an error in the Apeg parameter.

The $\zeta$ is a measure of the surface charge of LMNPs.(Figure 5-2(a)). LMNPs coated exclusively with DOTAP displayed the greatest $\zeta=58.5 \pm 7.8 \mathrm{mV}$, as expected. The $\zeta$ drops linearly between $5-25$ mol\% PEG $_{2000}$ from $37.0-31.2 \mathrm{mV}$ as PEG transitions from the mushroom-to-brush conformation. From 25-75 mol\% PEG, in the brush regime, the $\zeta$ drops steeply from 31.2 to $-22.2 \mathrm{mV}$. This drop is likely due to the rise in the anionic/cationic charge ratio and electrostatic screening from the PEG chains that extend away from the LMNP surface. The $\zeta$ plateaus at about $-22 \mathrm{mV}$ in the dense brush regime (75-100 mol\% PEG) when the anionic PEG completely masks 
the cationic lipid. The $\zeta$ is the electrostatic potential at the interface between the shear plane (where oppositely charged ions are bound) and the bulk solution. PEG can influence the $\zeta$ by displacing counter-ions, shifting the shear plane, and/or providing additional charge to the surface. ${ }^{21}$ The $\zeta$ changes with the distance away from the nanoparticle surface according to the following equation:

$$
\ln \zeta(L)=\ln A-\kappa \mathrm{L}
$$

where $\mathrm{L}$ is the position of the shear plans, $\mathrm{A}$ is the surface potential, and $\kappa$ is the inverse Debye length. ${ }^{3,22}$ Therefore, as the PEG surface density and/or distance away from the surface of the charged particle increases, the LMNP $\zeta$ decreases. ${ }^{23-26}$

Surface charge can be tuned by changing the LMNP lipid surface composition, as can be seen in Figure 5-2(a). The recorded $\zeta$ confirms that the lipid surface composition can be manipulated with the DSE method. Other groups have similarly demonstrated that the $\zeta$ of a nanoparticle can be manipulated by changing the concentration of differently charged surface lipids. ${ }^{27}$ The ability to control surface charge with DSE is important to making LMNPs capable of MRI contrast enhancement and siRNA delivery.

Cryo-TEM images of LMNPs with 0, 15, and 50 mol\% PEG-lipid are displayed in Figure 5-2(c). No structures can be seen on the surface of the DOTAP (0 mol\% PEG) LMNPs. In contrast, globule-like PEG structures can be seen in the 15 and $50 \mathrm{~mol} \%$ images extending from the LMNP surface. The $50 \mathrm{~mol} \%$ images show a higher density of PEG structures than 15 mol\%. PEG brush conformations are 
observed for $50 \mathrm{~mol} \%$ consistent with previous cryo-TEM observations for PEG brushes on block copolymer micelles. ${ }^{28}$

The ability to control the surface composition with the DSE method allows the benefits of PEGylation to be applied to SPIONs to form multifunctional LMNPs. Along with PEG's ability to improve circulation half-life, biodistribution, efficacy, and safety, it also has been shown to improve the $\mathrm{r}_{2}$ relaxivity allowing it to be used as a negative MRI contrast agent. Increasing concentrations of surface PEG has been show to increase the $\mathrm{r}_{2}$ relaxivitiy by thickening the hydration shell around the LMNPs. ${ }^{9} 10$ The effect of surface PEG concentration on $\mathrm{r}_{2}$ relaxivity of LMNPs is displayed in Figure 5-3(a). 

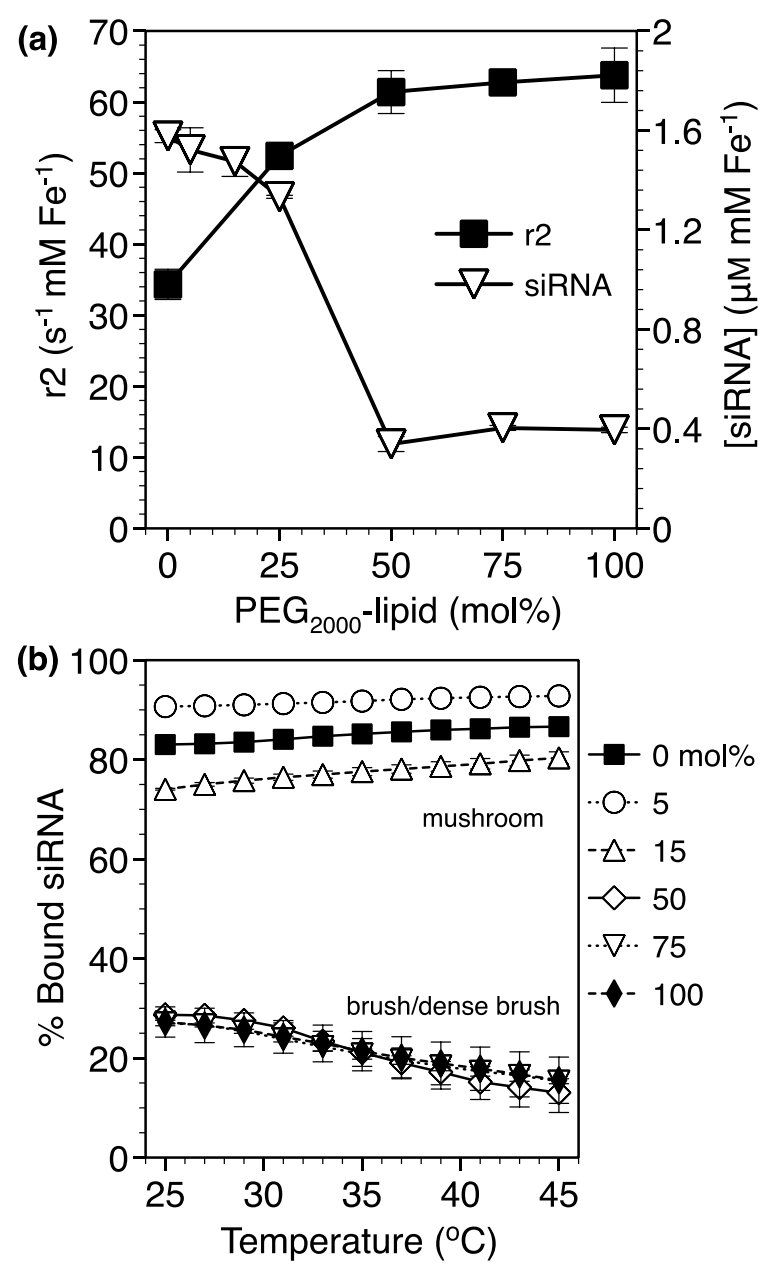

Figure 5-3. (a) Concentration of bound siRNA and $\mathbf{r}_{2}$ relaxivity and (b) percent of bound siRNA to LMNPs as a function of temperature.

(a) Concentration of bound siRNA and $\mathrm{r}_{2}$ relaxivity as a function of temperature (average \pm S.D.). (b) Percent of Bound siRNA to LMNPs as a Function of Temperature (average \pm S.D.)

The negatively charged phosphate backbone of siRNA electrostatically binds to the cationic charge provided by the DOTAP on the LMNP surface. The amount of siRNA that is bound to the LMNP surface was measured by a fluorescent quenching assay. The siRNA is labeled with a carboxyfluorescein (CF) fluorescent probe that self-quenches at concentrations greater than $30 \mu \mathrm{M} .{ }^{29}$ siRNA was shown to selfquench in the presence of LMNPs causing the fluorescent signal to fall. Figure 5-3(a) 
displays the effect of different siRNA concentrations on LMNPs. The trend in the amount of bound siRNA is likely caused by both the LMNPs being more cationic at lower mol\% PEG and the structural conformation of PEG. At lower PEG concentrations, the $\zeta$ is more cationic and there are less PEG chains to obstruct siRNA from bindng to the LMNP surface. Therefore, more siRNA could attach to the surface at lower mol\% PEG, but it attachment does not strictly follow $\zeta$.

The amount of siRNA bound to the LMNP surface was dependent on PEG conformation and temperature, as shown in Figure 5-3(b). In the mushroom conformation (0-15 mol\% PEG), more siRNA was able to bind to the LMNP, due to the hightened $\zeta$. PEG becomes more hydrophobic with increasing temperature causing the PEG to collapse and potentially interdigitate with the lipid monolayer. ${ }^{30}$ Therefore, in the mushroom conformation, as the temperature rises more cationic charges may be available for siRNA to bind to the LMNP due to the change in PEG as temperature increases. For the brush conformation (>15 mol\% PEG), the percent of bound siRNA also decreases with increasing temperature. Under these conditions, the temperature causes the PEG to become more hydrophobic, expelling siRNA from the surface. These results indicate that LMNPs can be used for the controlled release of siRNA.

LMNPs also can generate heat when subjected to alternating current electromagnetic field operating at radio frequency (RF), allowing them to be used for hyperthermia and/or triggered siRNA release. RF heating was performed using a $1 \mathrm{~kW}$ Hotshot $^{\mathrm{TM}}$ set to $300 \mathrm{~A}$ (actual output $\sim 227-235 \mathrm{~A}$ and $362 \mathrm{kHz}$ ) for $30 \mathrm{~min}$. The change in temperature from the recorded room temperature was recorded and is 
displayed in Figure 4(a). The heating capacity of nanoparticles in an RF is measured by the specific absorption rate (SAR):

$$
S A R=\frac{\Delta T}{\Delta t} \frac{c_{p} m_{V}}{m_{S P I O N}}
$$

where $c_{p}$ is the average heat capacity of the sample, in this instance water $(C=4.18$

$\mathrm{J} / \mathrm{g} \mathrm{K}), \mathrm{mv}$ is the mass of the sample volume, and msPION is the mass of the SPIONs. $\frac{\Delta T}{\Delta t}$ was calculated based on the initial slope of the heating curve. SAR for 15 and 50 mol\% PEG LMNPs were subjected were $52.8 \mathrm{~W} \mathrm{~g}^{-1}$ and $11.2 \mathrm{~W} \mathrm{~g}^{-1}$, respectively. This ability to heat when subjected to RF

The percent of siRNA released from the LMNP surface when subjected to RF heating is shown in Figure 5-4(b). The heating generated by the LMNPs caused the siRNA to release from the surface. At 50 mol\% PEG, the LMNPs release siRNA when subjected to RF heating. At 15 mol\% PEG, the LMNPs cause more siRNA to bind to the surface when subjected to RF heating, causing the percent of siRNA released to be negative. However, surprisingly, after $\sim 23$ min percent siRNA released begins to increase. Currently, it is unclear what mechanism causes this to happen.

In conclusion, LMNPs was created capable of being used as an MRI contrast agent and a temperature triggered siRNA delivery vehicle. LMNPs were coated with different compositions of cationic DOTAP and a methoxy-terminated PEG 2000 anchored to a DMPE lipid. The surface composition of LMNPs were controlled by a DSE method. LMNPs were shown to be multifunctional capable of increased MRI contrast and siRNA delivery. The MRI $\mathrm{r}_{2}$ relaxivity increased with increasing surface 
PEG concentration. LMNPs also demonstrated the ability to bind and release siRNA.

Release of siRNA was shown to be triggered by temperature and RF heating.
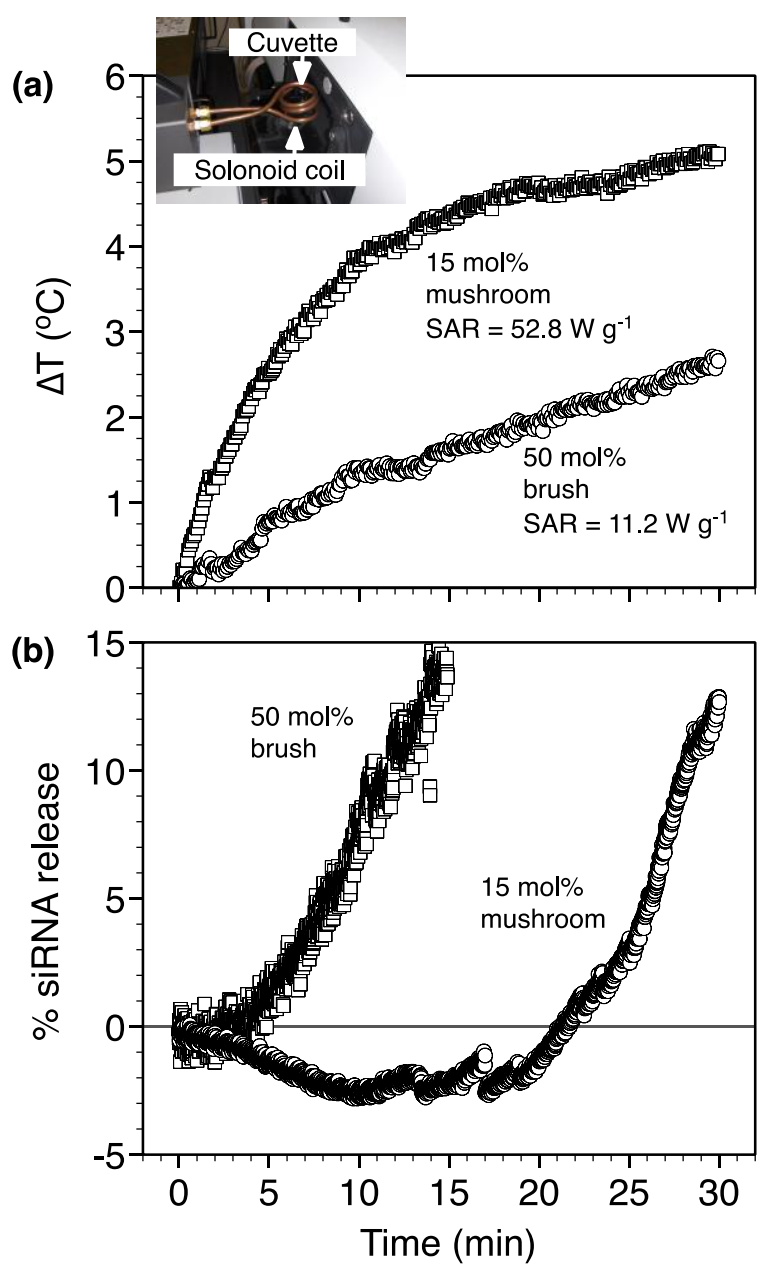

Figure 5-4. (a) Change in Bulk Sample Temperature over RF Heating Time and (b) Percent siRNA Release over RF Heating Time.

(a) Change in temperature from baseline temperature $\left(\sim 26^{\circ} \mathrm{C}\right)$ over $30 \mathrm{~min}$. when subjected to RF heating set to $300 \mathrm{~A}$ (actual output $\sim 226.8-235.4 \mathrm{~A}$ ) and $362 \mathrm{kHz}$. (b) Percent in bound siRNA over 30 min when subjected to RF heating set to $300 \mathrm{~A}$ (actual output $\sim 226.8-235.4 \mathrm{~A}$ ) and $362 \mathrm{kHz}$.

\section{Supporting Information}

Chemicals and Materials and Experimental Sections. 
The Experimental Section contains the following sections: Lipid Coated Magnetic Nanoparticle (LMNP) Formation, Lipid Coated Magnetic Nanoparticle (LMNP) Formation, Dynamic Light Scattering (DLS), Heating with Alternating Current Electromagnetic Field (AC EMF) at Radio Frequency (RF), Small Interfering RNA (siRNA) Experiments, and Magnetic Resonance Imaging (MRI).

The Small Interfering RNA (siRNA) Experiments contains the following subsections: siRNA Binding, siRNA Release with Temperature, and siRNA RF Release.

The Magnetic Resonance Imaging (MRI) section contains the following subsections: MRI Sample Preparation and MRI Methods for $\mathrm{r} 2$ Relaxation.

\section{Chemicals and Materials}

1,2-stearoyl-3-trimethylammonium-propane (DOTAP; $25 \mathrm{mg} / \mathrm{mL}$ ), 1,2dipalmitoyl-sn-glycero-3-phosphoethanolamine-N-(lissamine rhodamine B sulfonyl) (DMPE-PEG 2000;25 mg/mL), and 1,2-dipalmitoyl-sn-glycero-3-phosphoethanolamine$\mathrm{N}$-(lissamine rhodamine B sulfonyl) (rhodamine; $1 \mathrm{mg} / \mathrm{mL}$ ) lipids were purchased from Avanti Polar Lipids (Alabaster, AL USA). Oleic acid coated $30 \mathrm{~nm}$ superparamagnetic iron oxide nanoparticles were purc)hased from Ocean NanoTech (San Diego, CA USA). DMSO, agarose, 10X Tris-borate-EDTA (TBE) buffer, fetal bovine serum (FBS), Opti-MEM Reduced Serum Media, Dulbecco's Modified Eagle Medium (DMEM), and Vivaspin 2 100kDa MWCO Sample Concentrators from GE Health were purchased from Fisher Scientific (Suwanee, GA USA). FAM-labeled Negative Control siRNA (21 base pairs) was purchased from Shanghai GenePharma Co., Ltd. 
(Shanghai, China). Opti-MEM, DMEM, and FBS were purchased from Sterile deionized ultrafiltered (DI) water at $18.2 \mathrm{~m} \Omega$ was used from a Millipore Direct-Q3 UV purification system (Billerica, MA USA).

\section{Experimental Section}

\subsection{Lipid Coated Magnetic Nanoparticle (LMNP) Formation}

LMNPs were formed with a weight ratio of 32/1 lipid/Fe with a dual solvent exchange method derived from Tong, et. al. ${ }^{14}$ LMNPs with mole ratios of 100/0, 95/5, 85/15, 75/25, 50/50, 25/75, and 0/100 DOTAP/PEG and nanoparticles in chloroform were mixed together at desired concentrations in a round bottom flask. For rhodamine labeled LMNPs, rhodamine lipid was also added at a concentration of $0.6 \mathrm{~mol} \%$ of total lipids. DMSO, equal to 4 times the volume of chloroform, was then added to the flask in $50 \mu \mathrm{L}$ aliquots while sonicating in a $40 \mathrm{kHz}$ Branson Ultrasonics 1510 ultrasonic cleaner (Danbury, CT USA). The flask was them placed in a New Brunswick Scientific Excella E1 Platform Shaker (Enfield, CT USA) at a low-medium setting for $30 \mathrm{~min}$. Chloroform was removed by a BÜCHI Rotavapor R-215 rotoary evaporation (Flawil, Switzerland) at room temperature and 50 RPM at 200 mbar for 35 minutes and then $50 \mathrm{mbar}$ for $30 \mathrm{~min} .2-3 \mathrm{~mL}$ of DI water (at least equal to the volume of DMSO) was them added at $\sim 1 \mathrm{~mL}$ per 5-6 min. The sample was then placed in Vivaspin 2 Sample Concentrator tubes and centrifuged in a Thermo Scientific Megafuge 16R (Asheville, NC USA) at $20^{\circ} \mathrm{C}$ and $7000 \mathrm{~g}$ for $30 \mathrm{~min} .1 \mathrm{~mL}$ of DI water was then added to each tube using pipette mixing was used to remove any LMNPs from the membrane and then centrifuged again. This is repeated 2 more times 
before suspending the LMNPs in the 1-2 $\mathrm{mL}$ of DI water. To remove excess lipids and micelles, the samples were centrifuged at $18516 \mathrm{~g}$ for $15 \mathrm{~min}$. at room temperature. The supernatant was removed and discarded. The precipitate was resuspended in the desired volume of DI Water and redispersed by pipette mixing and vortexing.

\subsection{Cryogenic Transmission Electron Microscopy (Cryo-TEM)}

Iron oxide nanoparticles and LMNPs were examined with cryo-TEM imaging. $\sim 5 \mu \mathrm{L}$ of sample is deposited on a Quantifoil grid comprised of 200 square mesh copper grids suspended with $2 \mu \mathrm{m}$ carbon hole. (Electron Microscopy Sciences, Hatfield, PA, USA). Grids were robotically vitrified in liquid ethane using a Vitrobot (FEI Company, Hillsboro, OR USA). Prior to imaging, the vitrified grid is transferred and stored in liquid nitrogen. Imaging was performed in a liquid nitrogen cooled stage (Model 915, Gatan Inc., Pleasonton, CA USA) at $200 \mathrm{kV}$ using a JEOL JEM-2100F TEM (Peabody, MA USA).

\subsection{Dynamic Light Scattering (DLS)}

Hydrodynamic radius and zeta potential was investigated using a Malvern Zetasizer Nano ZS (Worcestershire, United Kingdom) equipped with a backscattering detector angle of $173^{\circ}$ and a $4 \mathrm{~mW}, 633 \mathrm{~nm} \mathrm{He}-\mathrm{Ne}$ laser. Hydrodynamicdiameters (dh) were measured using optical grade polystyrene cuvettes. Zeta potential was determined by combined Doppler electrophoretic velocimetry and phase analysis light scattering using folded capillary cells. 


\subsection{Heating with Alternating Current Electromagnetic Field (AC EMF) Operating at Radio Frequency (RF)}

RF heating was performed using a $1 \mathrm{~kW}$ Hotshot $^{\mathrm{TM}}$ (Ameritherm Inc., Scottsville, NY) set to 300 A (actual output $227-235 \mathrm{~A}$ and $362 \mathrm{kHz}$ ) with a 3 turn induction heating coil with a $3 \mathrm{~cm}$ outer diameter. A $3 \mathrm{~mL}$ plastic test tube was suspended in the center of the induction heating coils. $1 \mathrm{~mL}$ of $1 \mathrm{mg} / \mathrm{mL}$ of LMNPs were dispersed into the centrifuge tube. Temperature was taken with a LUXTRON ONE (LumaSense Technologies, Santa Clara, CA) $200 \mu \mathrm{m}$ all-silica with PFA Teflon jacket (RF immune) fiber optic probe. A hole was made at the center of the test tube cap to all the temperature probe to be placed at about the middle of the sample, while keeping the sample sealed. Temperature readings were recorded every $0.25 \mathrm{sec}$. with HyperTerminal. Sample temperature recording was then started. The sample was left at room temperature for $2 \mathrm{~min}$. to determine a baseline temperature. The sample was then subjected to RF heating for $30 \mathrm{~min}$. The specific absorption rates (SAR) of the nanoparticles were measured using a 3-turn copper coil with a $3 \mathrm{~cm}$ cm outer diameter. To determine SAR values, sample temperatures were measured from the initial slope of adiabatic temperature rise of the samples:

$$
S A R=\frac{\bar{c}_{p} m_{v}}{m_{N P}} \frac{\Delta T}{\Delta t}
$$




\section{Small Interfering RNA (siRNA) Experiments}

\section{1 siRNA Binding}

Fluorescently labeled siRNA enabled LMNP binding to be investigated with a fluorescent quenching assay. Fluorescent measurements were taken with a PerkinElmer Model LS 55 fluorescence spectrometer (Waltham, MA USA) with an excitation and emissions slit width of $10 \mathrm{~nm}$, a $1 \%$ attenuation filter, and excitation and emission wavelength of $494 \mathrm{~nm}$ and $516 \mathrm{~nm}$, respectively. Opti-MEM was added to a quartz cuvette and the intensity, IoptimEm, was recorded. siRNA was then added and the intensity, IsiRNA/OptiMEM, was recorded. Finally, LMNPs were added and the intensity, $\mathrm{I}(\mathrm{t})$, was integrated over $10 \mathrm{sec}$. intervals and recorded over $15 \mathrm{~min}$. The percent of bound siRNA was calculated with the following equation:

$$
\text { Bound siRNA } \%=\frac{\left(I_{\text {SiRNA/OptiMEM }}-I(t)\right)}{\left(I_{\text {SiRNA/OptimEM }}-I_{\text {OptiMEM }}\right)}
$$

To ensure that quenching was due to LMNPs only, samples were then transferred to centrifuge tubes and centrifuged at $6000 \mathrm{~g}$ for $15 \mathrm{~min}$. The supernatant was then transferred to a quartz cuvette and 3 scans were taken.

\section{2 siRNA Release with Temperature}

Intensity readings were taken similar to above. Opti-MEM was added to a quartz cuvette and the intensity, IoptimEm, was recorded. siRNA was then added and the intensity, IsiRNA/OptiMEM, was recording. LMNPs were then added and intensity readings 
were recorded between $25^{\circ}-45^{\circ} \mathrm{C}$. Temperature was ramped at $2^{\circ} \mathrm{C}$ and after holding the temperature for $5 \mathrm{~min}$. the intensity, I(T), was recorded. The percent of bound siRNA was calculated with the following equation:

$$
\text { Bound siRNA } \%=\frac{\left(I_{\text {SiRNA/OptimEM }}-I(T)\right)}{\left(I_{\text {SiRNA/OptiMEM }}-I_{\text {OptiMEM }}\right)}
$$

\section{3 siRNA RF Release}

siRNA RF release experiments were conducted with a copper heating coil (3 turns at $4.5 \mathrm{~cm}$ mean diameter) around a custom-designed polycarbonate cuvette holder and base of the fluorescent spectrometer. The amount of bound siRNA before RF heating was calculated with the following equation:

$$
\text { Bound SiRNA } \%=\frac{\left(I_{\text {SiRNA/OptimEM }}-I_{\text {LMNP/SiRNA/OptiMEM }}\right)}{\left(I_{\text {SiRNA/OptimEM }}-I_{\text {OptiMEM }}\right)}
$$

Opti-MEM was added to a quartz microcuvette and the intensity was taken for $2 \mathrm{~min}$. The intensity, Ioptimem, was the average of the last 30 secs. Then, $2.0 \mu \mathrm{L}$ of siRNA $(16.67 \mu \mathrm{M})$ was added and the intensity taken for $2 \mathrm{~min}$. The intensity, IsiRnA/OptiMEM, was the average of the last $30 \mathrm{sec}$. Lastly, $41.2 \mu \mathrm{L}$ of LMNP $\left(1.25 \times 10^{12} \mathrm{LMNP} / \mathrm{mL}\right)$ was added and the intensity taken for $2 \mathrm{~min}$. The intensity, ILMNP/siRnA/OptiMEM, was the average of the last $30 \mathrm{sec}$. The microcuvette was subjected to RF heating for $10 \mathrm{~min}$. RF heating was conducted within a $1 \mathrm{~kW}$ Hotshot (Ameritherm Inc., Scottsville, NY USA) operating at a setting of $300 \mathrm{~A}$ and $270-281 \mathrm{kHz}$. 


\section{Magnetic Resonance Imaging (MRI)}

\subsection{MRI Sample Preparation}

LMNPs were suspended in $1 \%$ agarose at concentrations of 1,10 , and 20 $\mu \mathrm{g} / \mathrm{mL} \mathrm{Fe}_{3} \mathrm{O}_{4}$ in $4 \mathrm{~mL}$ plastic sample tubes. $1 \%$ agarose gels were prepared by mixing agarose with 10X TBE buffer (diluted to $1 \mathrm{X}$ ) in a flask on a hot plate set to keep the gel at $80^{\circ} \mathrm{C}$ until the agarose is completely dissolved. The gel and LMNP sample was then pipetted into a sample holder, vortexed, and stored at $4^{\circ}-8^{\circ} \mathrm{C}$ until imaged.

\subsection{MRI Methods for $\mathbf{r}_{2}$ Relaxation}

Samples were imaged using a Siemens Prisma 3T scanner. The sample holder was placed in a 64 channel head receive array. LMNP samples in $4 \mathrm{ml}$ vials were scanned using spin echo $\left(\mathrm{r}_{2}\right)$. Cross section images of the vials were obtained with voxel size of $0.78 \mathrm{~mm}$ and slice thickness of $4 \mathrm{~mm}$. Repetition time was $2400 \mathrm{~ms}$ for all sequences. For the spin echo acquisition 24 echoes were collected over the range of 9 $-216 \mathrm{~ms}$ (9ms step). The inversion recovery data were taken with inversion times of 100, 200, 300, 400, 600, 1000, and 1500ms. Relaxation time constants were determined using a nonlinear least squares fit for pixel intensity vs echo time for $\mathrm{r}_{2}$. Three-parameter nonlinear least squares fitting routines $\left(\mathrm{M}_{0}, \mathrm{~T}_{1,2}\right.$. DC offset) were used for $\mathrm{r}_{2}$ to take into account through-slice dephasing effects ${ }^{31}$. Relaxivity was calculated as a linear fit of relaxation rates to iron concentration. 


\subsection{Acknowledgements}

This research was sponsored by CBET-1337061. We gratefully acknowledge Dr. Yanjing Chen, Dr. Everett Crisman, Dr. Samantha Meenach, Yaser Kashcooli, Andrea Richard Kingsley, T. Ryan Lynn, Karissa Paquin, Alexandra Tsoras, Andrea Villarroel, and Elizabeth Vuono for their assistance, advice, and support on this project.

\section{References}

1. Rudolf, H.; Silvio, D.; Robert, M.; Matthias, Z. Journal of Physics: Condensed Matter 2006, 18, (38), S2919.

2. $\quad$ Perry, J. L.; Reuter, K. G.; Kai, M. P.; Herlihy, K. P.; Jones, S. W.; Luft, J. C.; Napier, M.; Bear, J. E.; DeSimone, J. M. Nano letters 2012, 12, (10), 5304-10.

3. Rabanel, J. M.; Hildgen, P.; Banquy, X. Journal of controlled release : official journal of the Controlled Release Society 2014, 185, 71-87.

4. Moghimi, S. M.; Szebeni, J. Progress in lipid research 2003, 42, (6), 463-78.

5. Kanasty, R.; Dorkin, J. R.; Vegas, A.; Anderson, D. Nature materials 2013, $12,(11), 967-77$.

6. Immordino, M. L.; Dosio, F.; Cattel, L. International Journal of Nanomedicine 2006, 1, (3), 297-315.

7. Vonarbourg, A.; Passirani, C.; Saulnier, P.; Benoit, J.-P. Biomaterials 2006, 27, (24), 4356-4373.

8. Owens Iii, D. E.; Peppas, N. A. International Journal of Pharmaceutics 2006, 307, (1), 93-102. 
9. Tong, S.; Hou, S.; Zheng, Z.; Zhou, J.; Bao, G. Nano letters 2010, 10, (11), 4607-13.

10. LaConte, L. E.; Nitin, N.; Zurkiya, O.; Caruntu, D.; O'Connor, C. J.; Hu, X.; Bao, G. Journal of magnetic resonance imaging : JMRI 2007, 26, (6), 163441.

11. Whitehead, K. A.; Langer, R.; Anderson, D. G. Nature reviews. Drug discovery 2009, 8, (2), 129-38.

12. Kanasty, R. L.; Whitehead, K. A.; Vegas, A. J.; Anderson, D. G. Molecular therapy: the journal of the American Society of Gene Therapy 2012, 20, (3), $513-24$

13. Zhang, S.; Zhao, B.; Jiang, H.; Wang, B.; Ma, B. Journal of controlled release : official journal of the Controlled Release Society 2007, 123, (1), 1-10.

14. Tong, S.; Hou, S.; Ren, B.; Zheng, Z.; Bao, G. Nano letters 2011, 11, (9), $3720-6$.

15. Damodaran, V. B.; Fee, C. J.; Ruckh, T.; Popat, K. C. Langmuir 2010, 26, (10), 7299-7306.

16. Rädler, J. O.; Koltover, I.; Jamieson, A.; Salditt, T.; Safinya, C. R. Langmuir 1998, 14, (15), 4272-4283.

17. Ohki, S., Molecular Mechanisms of Membrane Fusion. Springer US: 2012.

18. Sukhishvili, S. A.; Chen, Y.; Muller, J. D.; Gratton, E.; Schweizer, K. S.; Granick, S. Nature 2000, 406, (6792), 146-146.

19. Sukhishvili, S. A.; Chen, Y.; Müller, J. D.; Gratton, E.; Schweizer, K. S.; Granick, S. Macromolecules 2002, 35, (5), 1776-1784. 
20. Ma, Y.; Tong, S.; Bao, G.; Gao, C.; Dai, Z. Biomaterials 2013, 34, (31), 77067714.

21. Ostolska, I.; Wiśniewska, M. Colloid and Polymer Science 2014, 292, (10), 2453-2464.

22. Sadzuka, Y.; Nakade, A.; Hirama, R.; Miyagishima, A.; Nozawa, Y.; Hirota, S.; Sonobe, T. International Journal of Pharmaceutics 2002, 238, (1-2), 171180.

23. Meng, F.; Engbers, G. H.; Feijen, J. Journal of Biomedical Materials Research Part A 2004, 70, (1), 49-58.

24. Rahme, K.; Chen, L.; Hobbs, R. G.; Morris, M. A.; O'Driscoll, C.; Holmes, J. D. $R S C$ Advances 2013, 3, (17), 6085-6094.

25. Gref, R.; Miralles, G.; Dellacherie, E. Polymer international 1999, 48, (4), 251-256.

26. Stolnik, S.; Dunn, S. E.; Garnett, M. C.; Davies, M. C.; Coombes, A. G.; Taylor, D.; Irving, M.; Purkiss, S.; Tadros, T. F.; Davis, S. S. Pharmaceutical research 1994, 11, (12), 1800-1808.

27. Xu, F.; Reiser, M.; Yu, X.; Gummuluru, S.; Wetzler, L.; Reinhard, B. M. ACS nano 2016, 10, (1), 1189-200.

28. Fenyves, R.; Schmutz, M.; Horner, I. J.; Bright, F. V.; Rzayev, J. Journal of the American Chemical Society 2014, 136, (21), 7762-70.

29. Torchilin, V. P.; Weissig, V., Liposomes : a practical approach. 2 nd ed.; Oxford University Press: Oxford ; New York, 2003; p xxiii, 396 p. 
30. Branca, C.; Magazù, S.; Maisano, G.; Migliardo, F.; Migliardo, P.; Romeo, G. The Journal of Physical Chemistry B 2002, 106, (39), 10272-10276.

31. Fernández-Seara, M. A.; Wehrli, F. W. Magnetic Resonance in Medicine 2000, 44, (3), 358-366. 